\title{
High Energy Astrophysics
}

I.

Cosmic Rays

\& Neutrinos

Péter Mészáros

Pennsylvania State University

NIC20 I 4, Debrecen, 


\section{What are Cosmic Rays?}

- Early I900s: Electroscopes near radioactive radiation sources discharge; at rate which is proportional to the radiation intensity

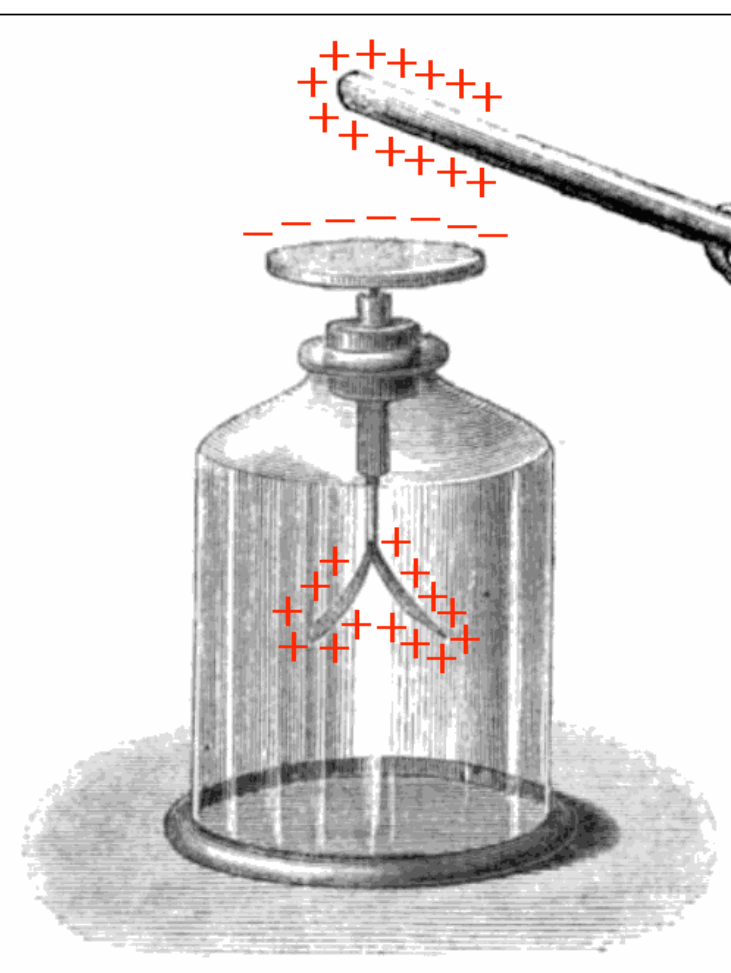

- However, even far from radioactive sources, the electroscope discharges slowly $\rightarrow$ some other source of radiation- but what?

- 1910-1913: various experiments try to identify sources. Italian physicist Domenico Pacini $\rightarrow$ if descend below the sea:

$\rightarrow$ radiation intensity decreases with depth!

- Austrian physicist Victor Hess, I 9 | I - | 3: go up in balloons $\leq \mathbf{5} \mathbf{~ k m}$ altitude: radiation intensity first decreases for first $\mathrm{km}$, but then increases with altitude ! (..comes from above ?!!) 


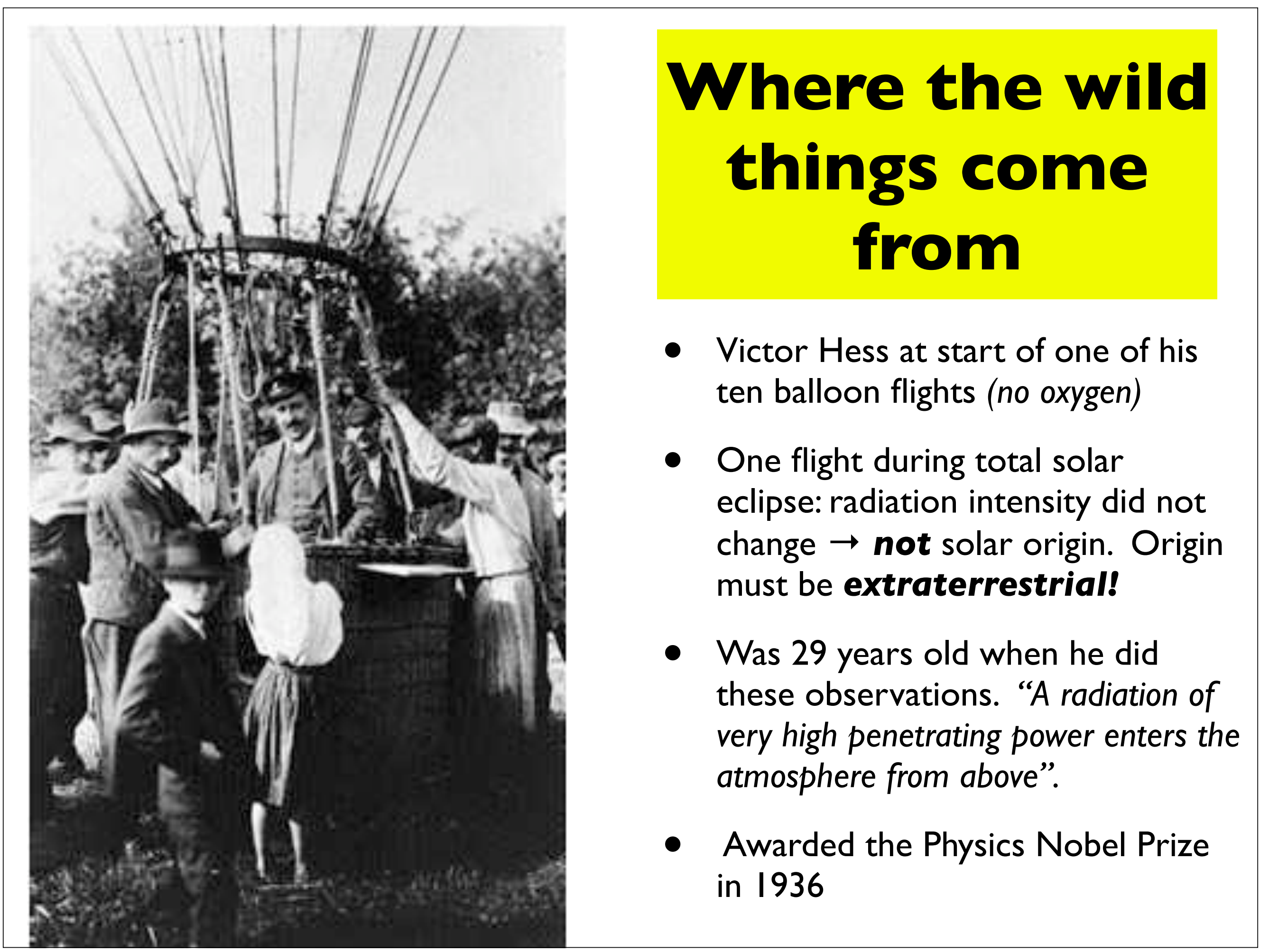




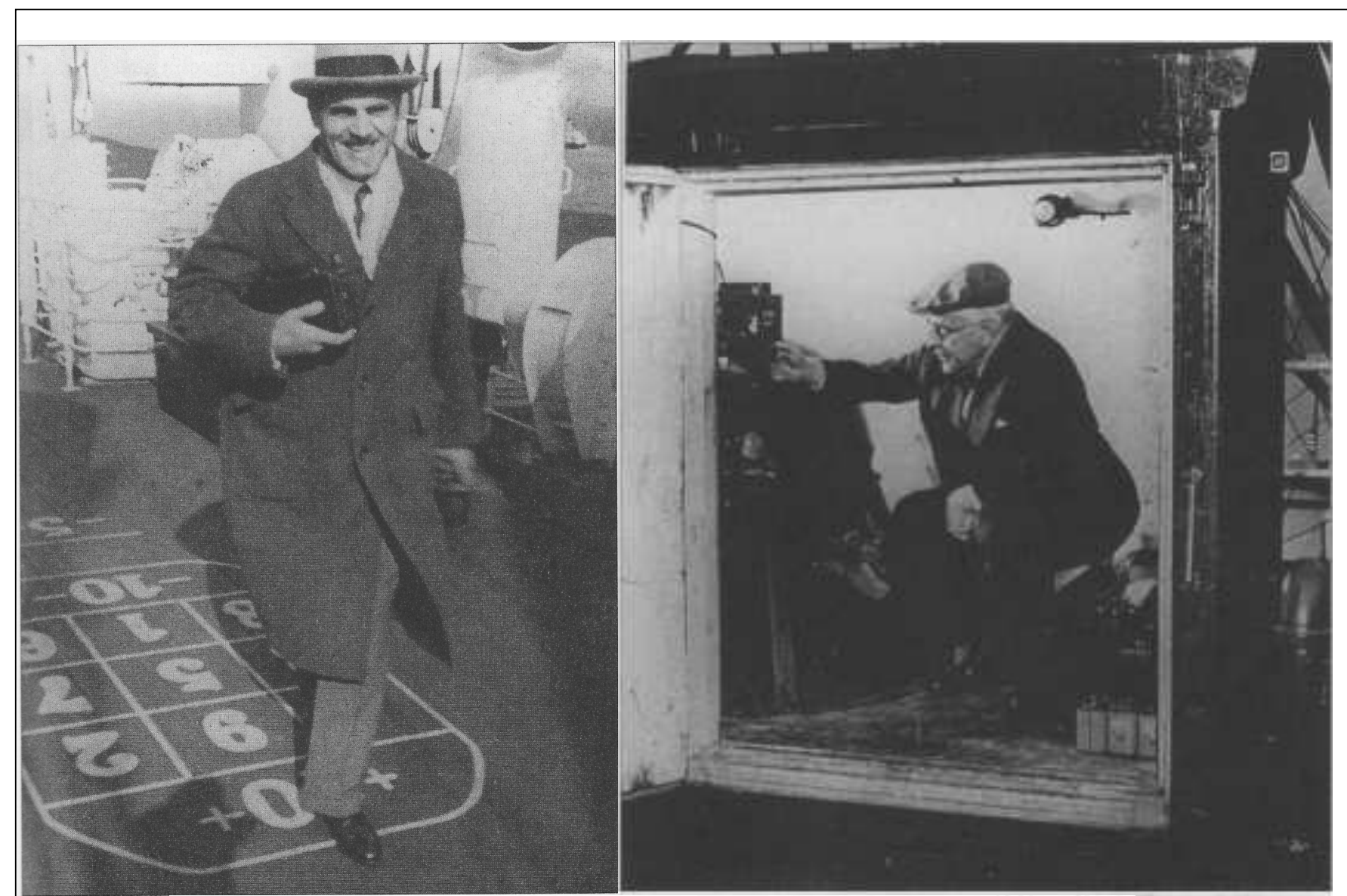

Mounting Clues

- A.H. Compton measured cosmic ray rates around the world (1935)

- Lemaitre,Vallarta, Johnson, Alvarez: incoming CRs follow Earth's geomagnetic latitude

- East-West asymmetry: the parent cosmic rays must be (positively) charged particles

[ CR slides credit: Stephane Coutu ] 


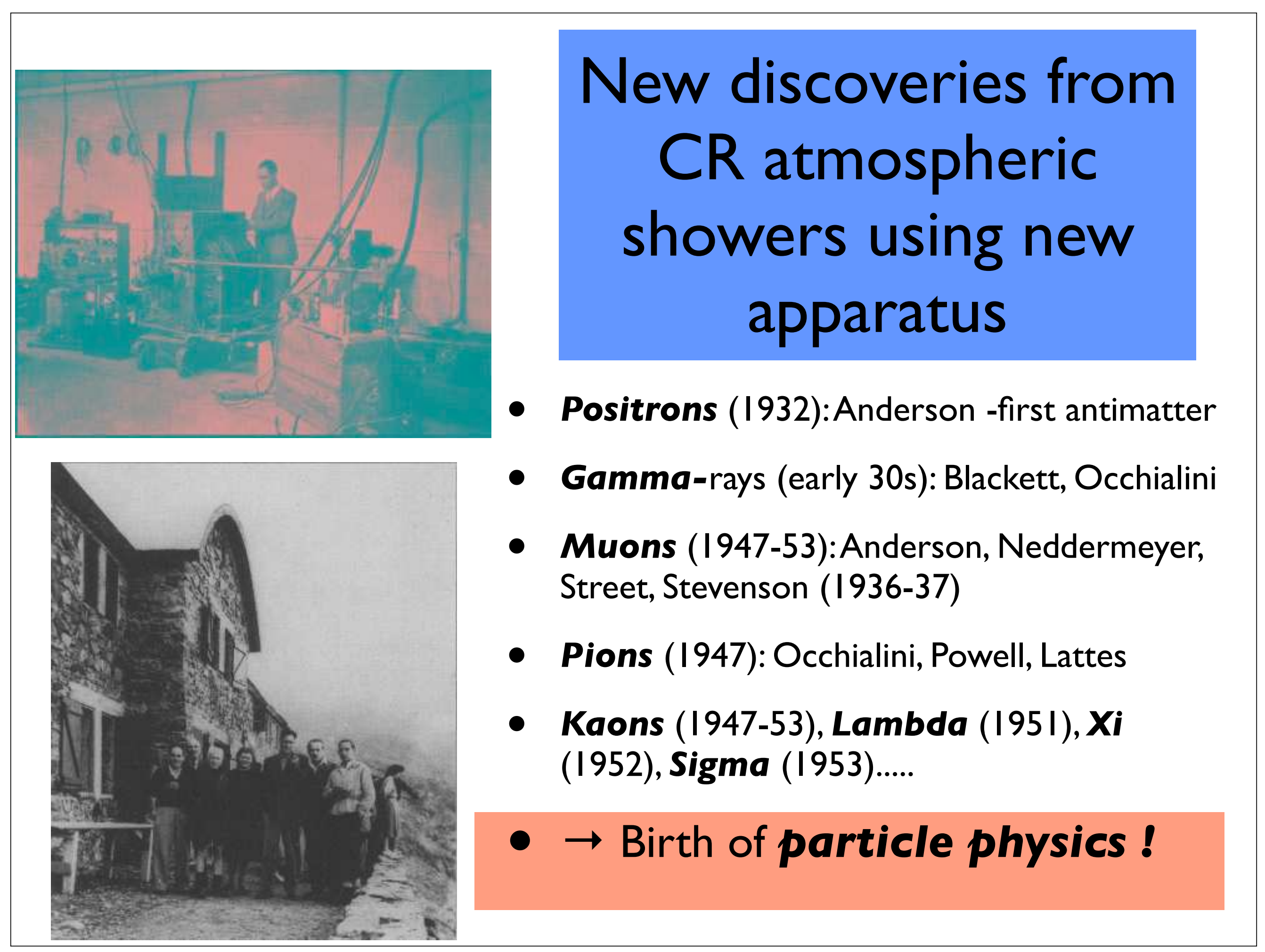




\section{Further progress}

Pierre Auger discovers extensive air

showers in 1938

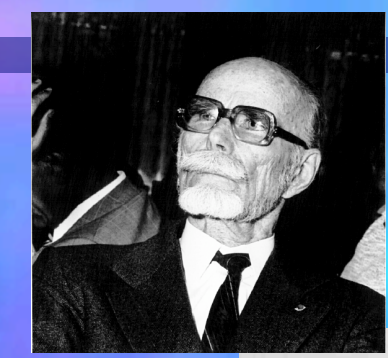

[ CR slides (blue bkg.) credit: Stephane Coutu ]

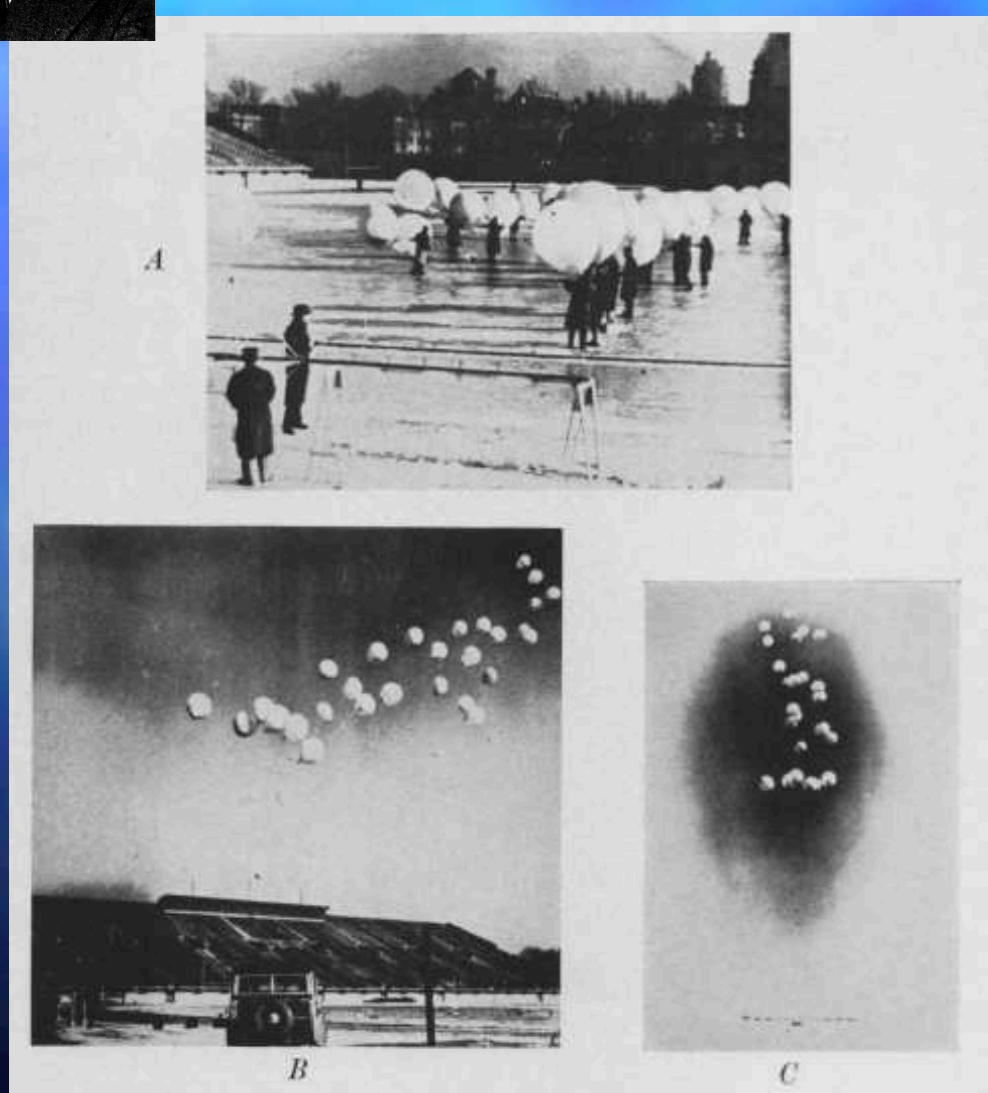

Used balloons too (automated)
So far, all particles seen are made in the atmosphere...
Cosmic rays can be extraordinarily energetic $>10^{15} \mathrm{eV}$ (millions of times the energy in the mass of a proton).

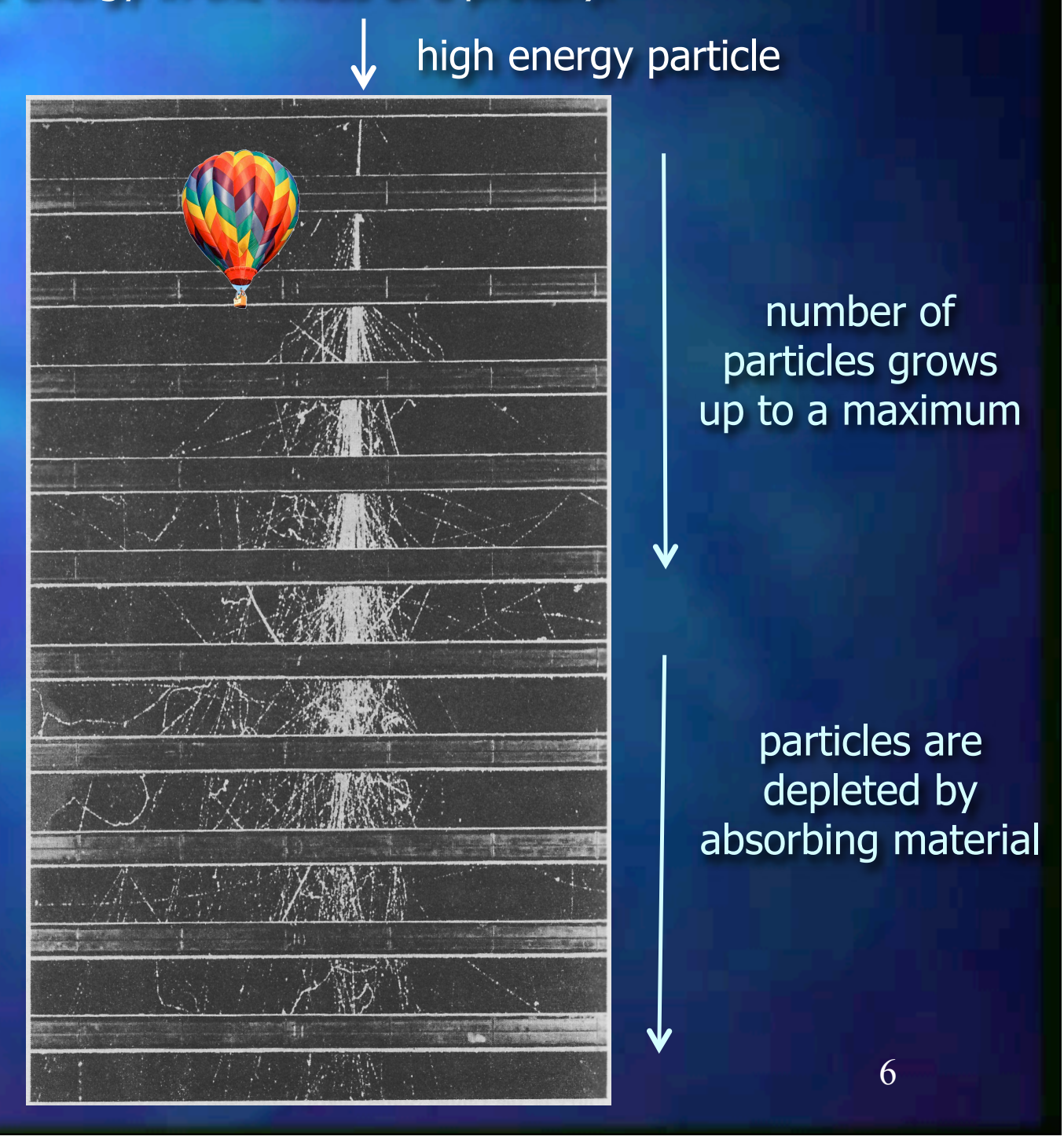




\section{Direct measurements}

Starting in late 1940s, unmanned balloons up to $130,000 \mathrm{ft}$, up to tens of hours; direct measurements of cosmic particles become possible; primary cosmic rays are $85 \%$ protons (1940s); there are nuclei too, 12\% helium, 2\% Li-Fe (1948-50); there are $1 \%$ electrons too (1961).
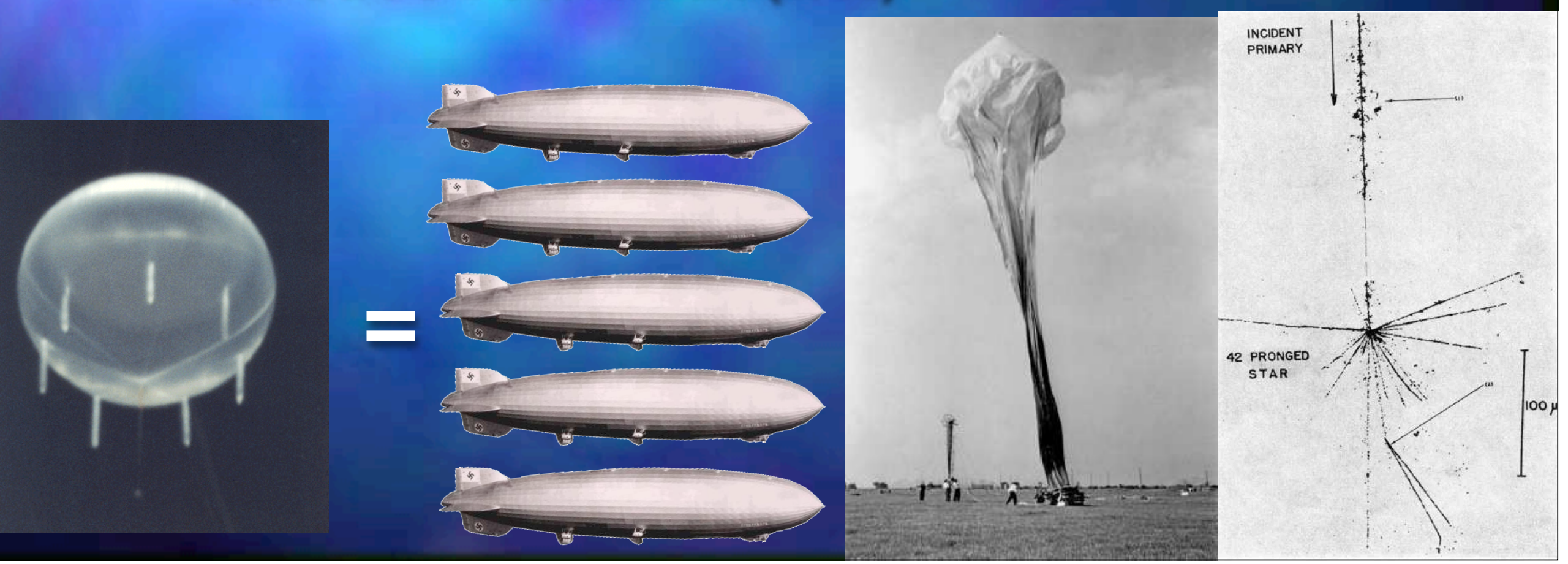


\section{Balloonjing in the news}

Stratolab 5, May 4, 1961

114 kft (Malcolm Ross ok, Vic Prather drowned); next day: Alan Shepard on Mercury Freedom 7

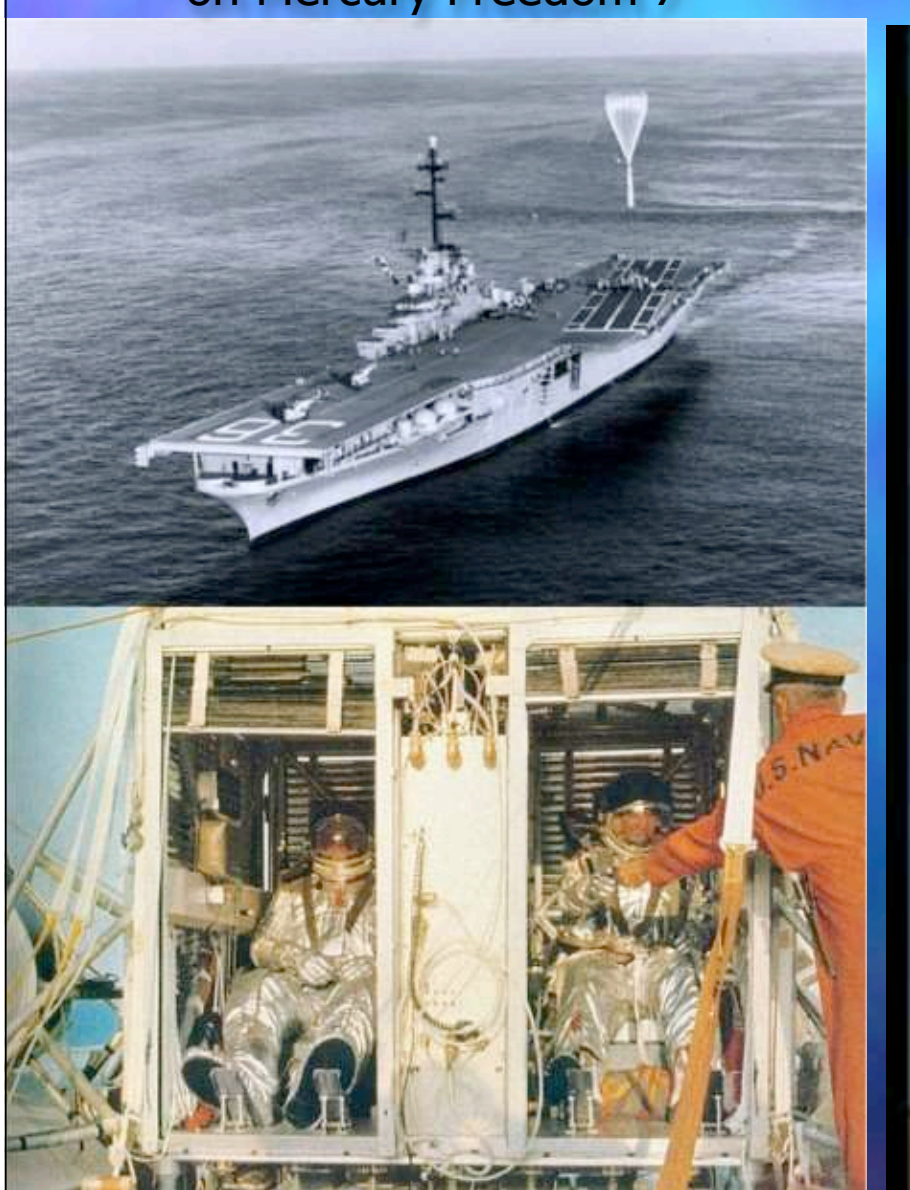

Nuclear Compton Telescope

NCT, Alice Springs, Apr 29, 2010 :

the dangers of science .....

Courtesy Catalyst
WABCNews 


\section{Direct measurements}

Since 1987: launches from McMurdo, Antarctica; flights up to 42 days!

\section{NASA/Columbia Scientific Balloon Facility (CSBF)}
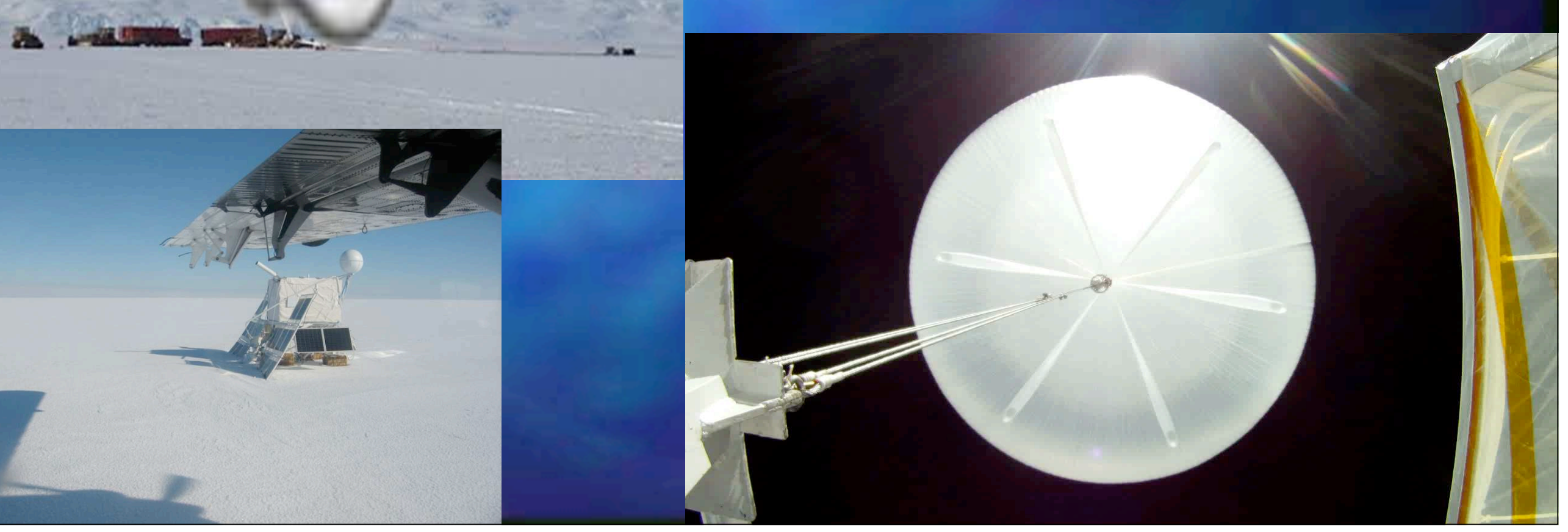


\section{Gettjing to Antarctica}

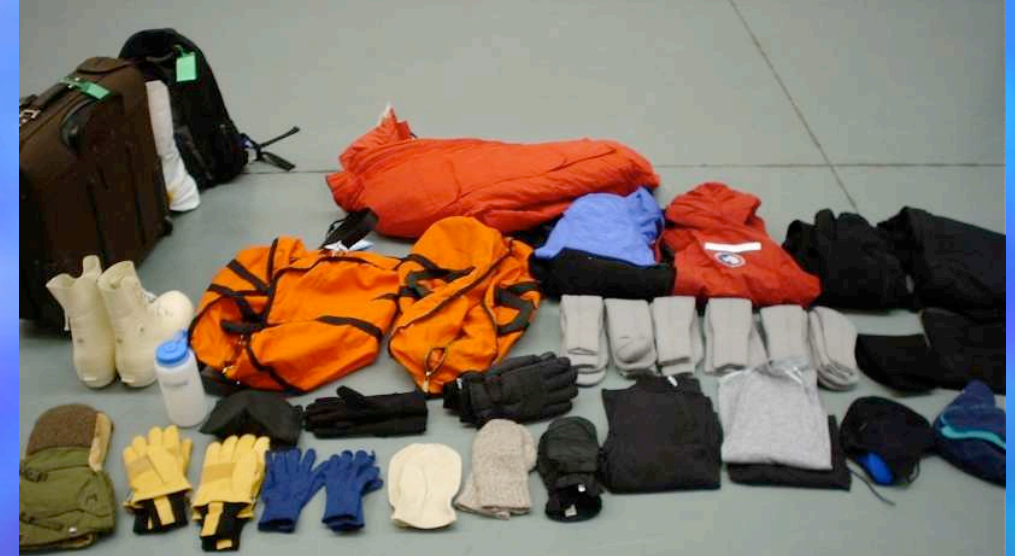

* McMurdo
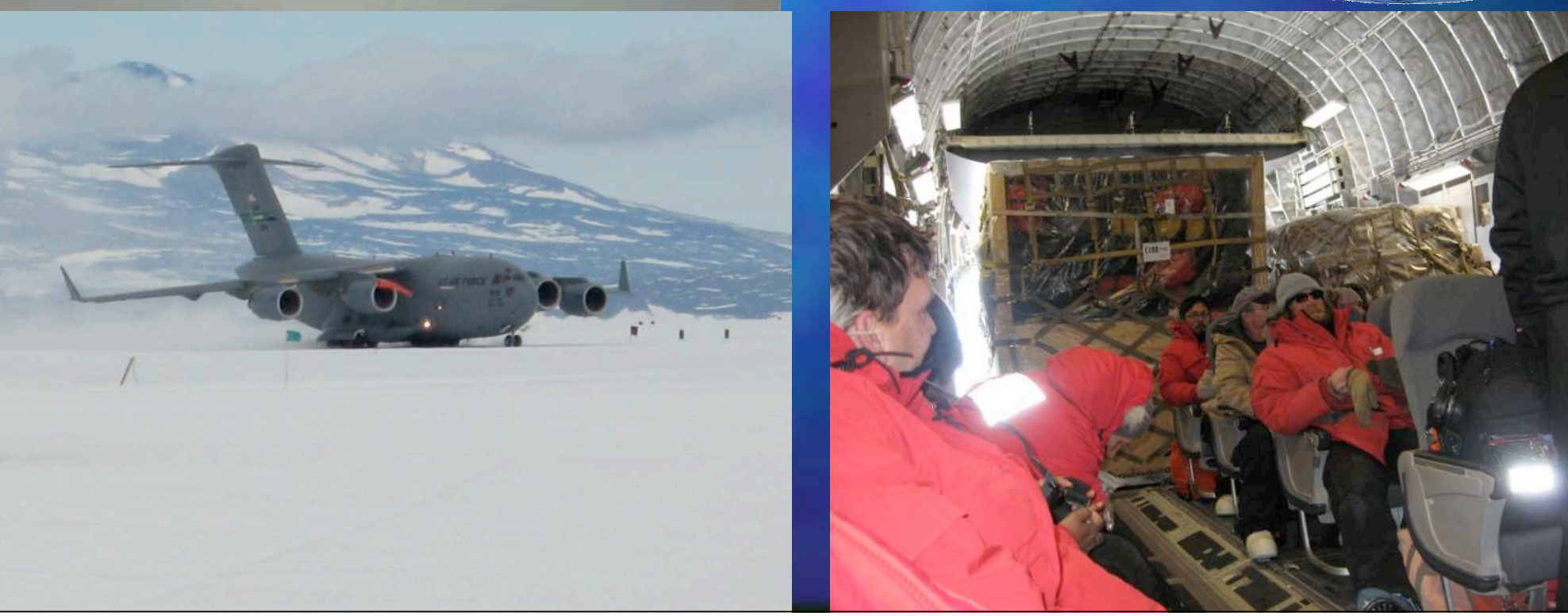


\section{McMuJido}

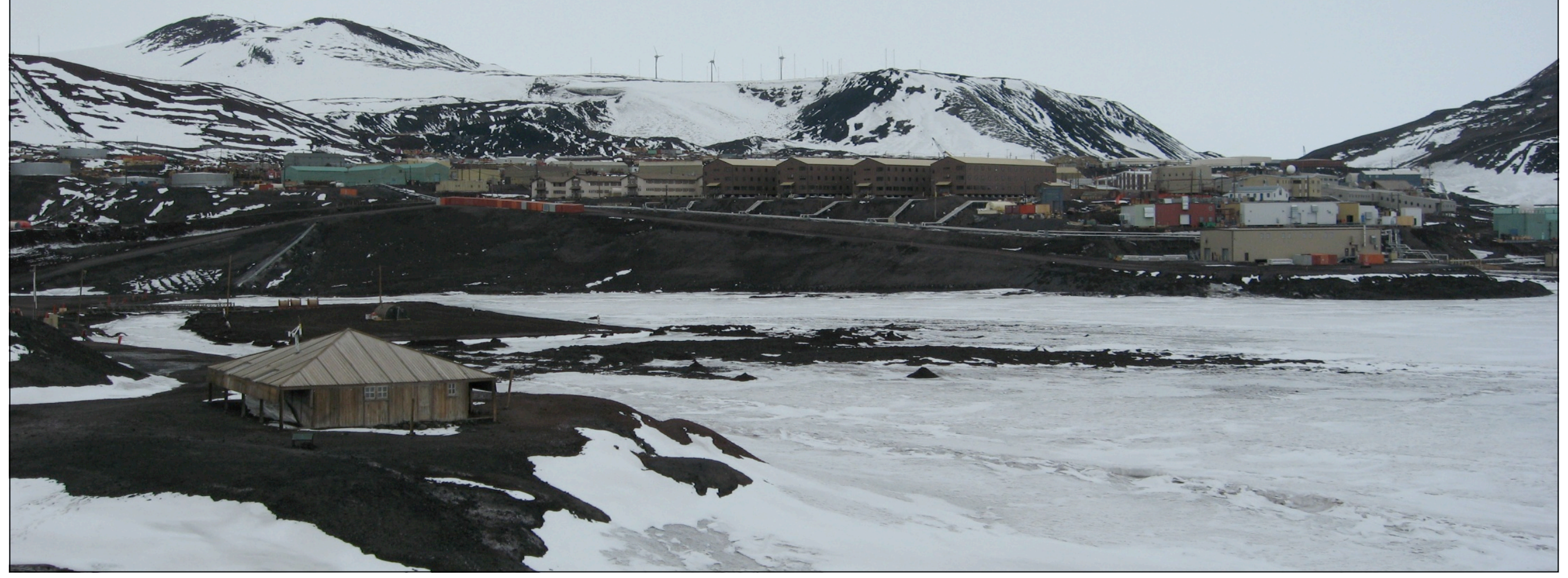




\section{CREAM \\ (Cosmj.c Ray Energetics And Mass)}

Since the 1960 s, ever larger, more complex instruments flown on large balloons for longer durations;

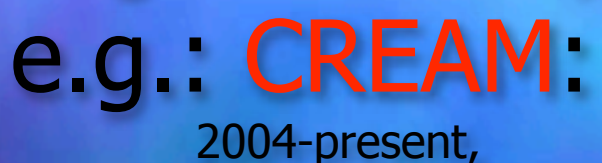

2004-present,

6 Antarctic flights, 160 days of exposure, flight 7 in Dec 2013.

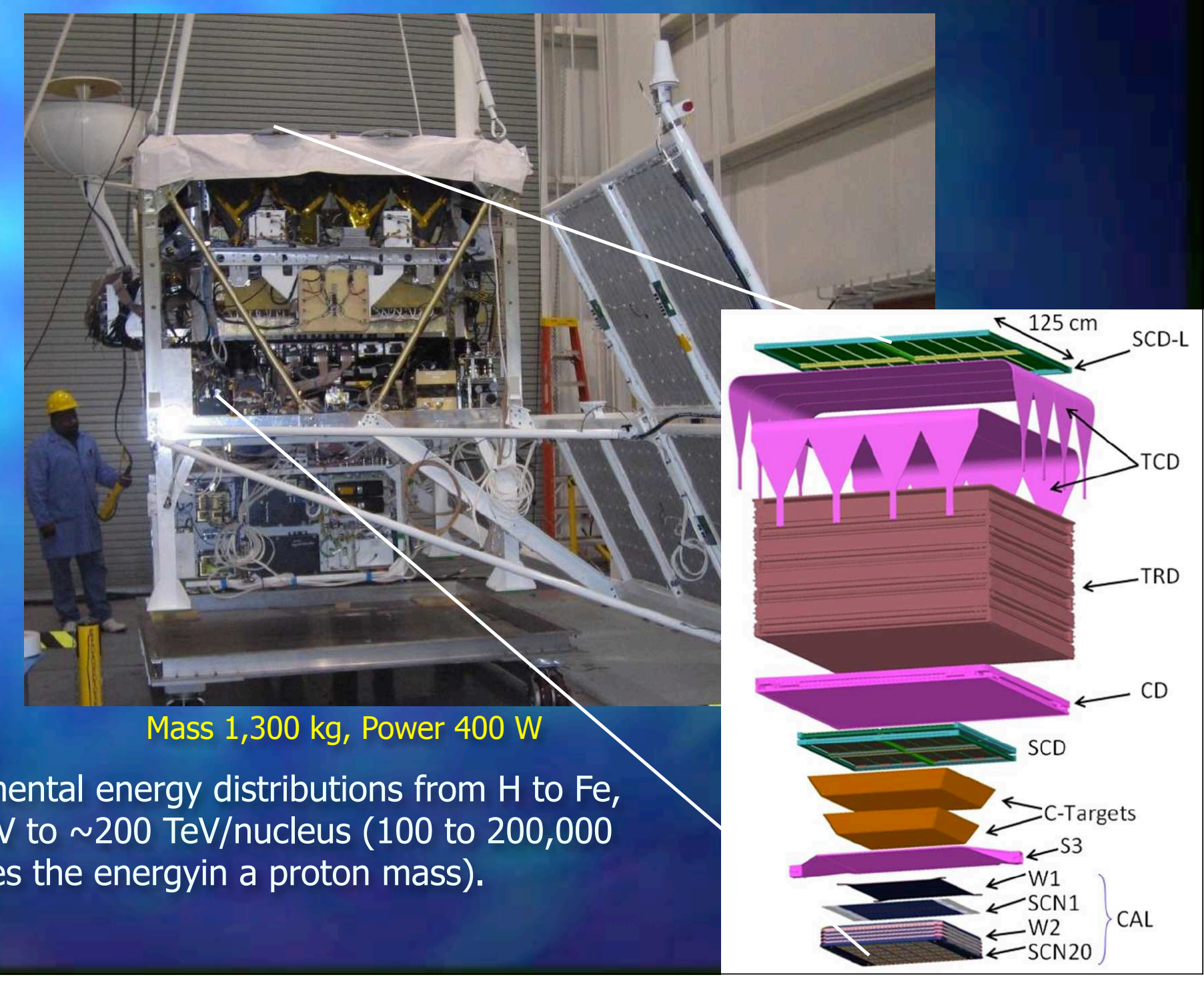

Measure elemental energy distributions from $\mathrm{H}$ to $\mathrm{Fe}$, from $100 \mathrm{GeV}$ to $\sim 200 \mathrm{TeV} /$ nucleus (100 to 200,000 times the energyin a proton mass). 


\section{Elenental abundances}

Energy $1 \mathrm{GeV} / \mathrm{n}$ to $4 \mathrm{TeV} / \mathrm{n}$, unmatched charge resolution $(\sim 0.2 \mathrm{e})$ in this energy regime.

Ahn H.S. et al., ApJ 714, L89 (2010)

C primary, but B arises from spallation in interstellar collisions...

$\mathrm{B} / \mathrm{C}$ tells the history of propagation (over 7-8 million years).

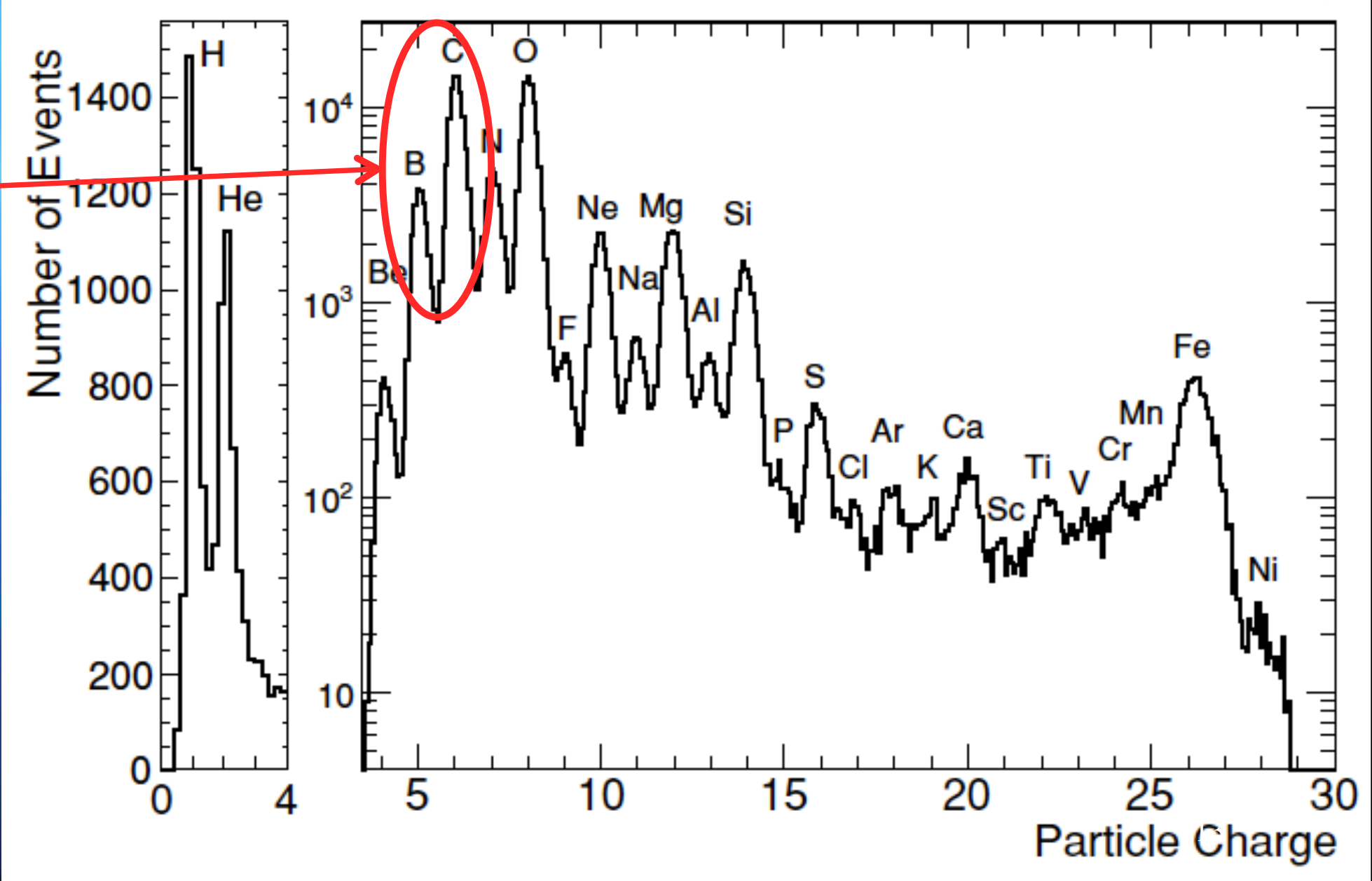




\section{What accelerates $C R s$ to these high energies?}

1940s :

$\mathrm{E}_{\mathrm{CR} \sim \mathrm{Pev}}$ $\left(10^{15} \mathrm{eV}\right)$ 


\section{What accelerates CRs to these high energies?}

\section{0s :}

$\mathbf{E}_{\mathrm{CR} \sim \mathrm{Pev}}$ $\left(10^{15} \mathrm{eV}\right)$

\section{Enrico Fermi}

Initially proposed scattering of CRs, bouncing off the magnetic field of interstellar clouds, (Fermi's 2nd order mechanism), effic. $\Delta \mathrm{E} / \mathrm{E} \sim(\mathrm{V} / \mathrm{c})^{\mathbf{2}} /$ scatt. $^{-}$ 


\section{What accelerates CRs to these high energies?}

1940s : $\mathbf{E}_{\mathrm{CR} \sim \mathrm{Pev}}$ $\left(10^{15} \mathrm{eV}\right)$

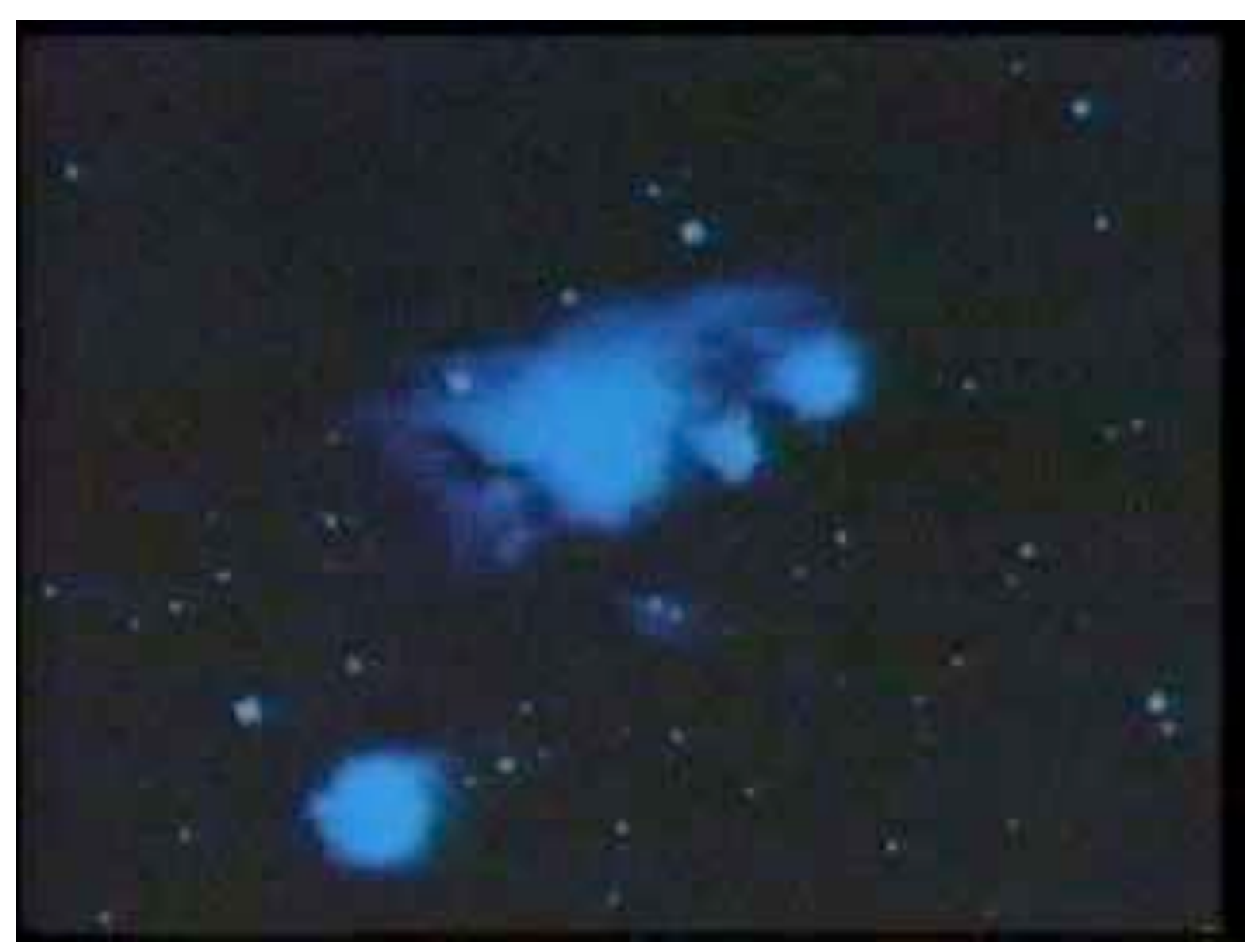

\section{Enrico Fermi}

Initially proposed scattering of CRs, bouncing off the magnetic field of interstellar clouds, (Fermi's 2nd order mechanism), $\Delta \mathrm{E} / \mathrm{E} \sim(\mathrm{V} / \mathrm{c})^{2} /$ scatt. 


\section{What accelerates CRs to these high energies?}

1940s : $\mathbf{E}_{\mathrm{CR}} \sim \mathrm{Pev}$ $\left(10^{15} \mathrm{eV}\right)$

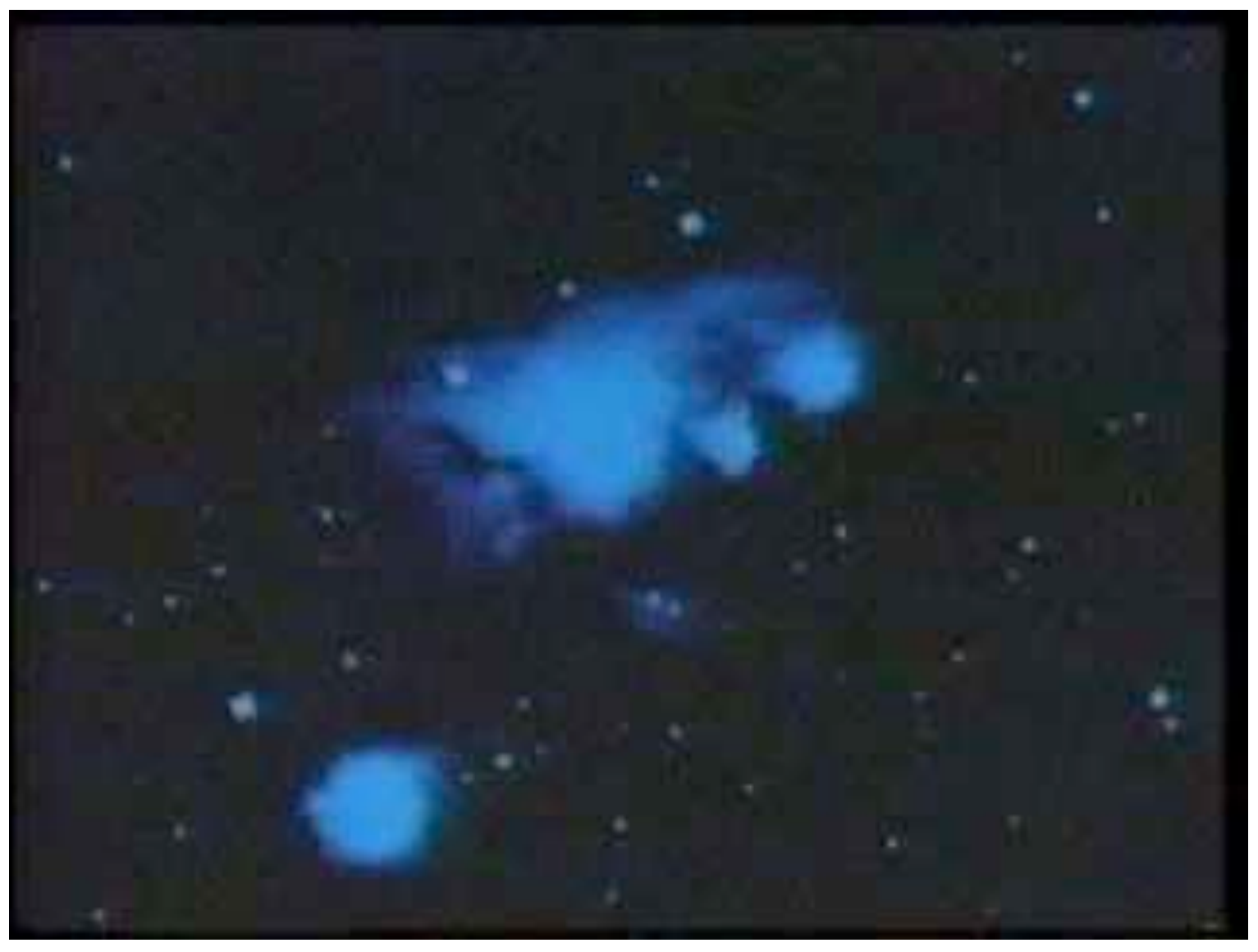

\section{Enrico Fermi}

Initially proposed scattering of CRs, bouncing off the magnetic field of interstellar clouds, (Fermi's 2nd order mechanism), $\Delta \mathrm{E} / \mathrm{E} \sim(\mathrm{V} / \mathrm{c})^{2} / \mathrm{scatt}$. BUT: mechanism not very efficient 


\section{Fermi's next try: 1st order mech., $\Delta E / E \sim(V / c)$}

Ground-based

Supernova remnants

are a candidate for

Galactic cosmic

ray

sources

Enrico Fermi (1940s) shock acceleration, naturally predicts

$E^{-2.1}$ at the source $\Rightarrow \sim E^{-2.6}$ at Earth

\section{of SNRS support} Chandra Hubble

$$
E_{\max } \approx \beta c Z e B L
$$




\section{Space measurements}

Also can fly instruments on rockets and satellites e.g.:

- 1979: HEAO-3 Atlas-Centaur rocket; - 1985: CRN Space Shuttle Challenger; 1997: ACE Delta II rocket; 2011: AMS International Space Station.

Long exposures (years), no residual atmospheric overburden, true vacuum, power from solar panels; but takes years (decades?) of development, testing, qualification, and can be very expensive (e.g., AMS cost is estimated at \$2B - \$4B)...

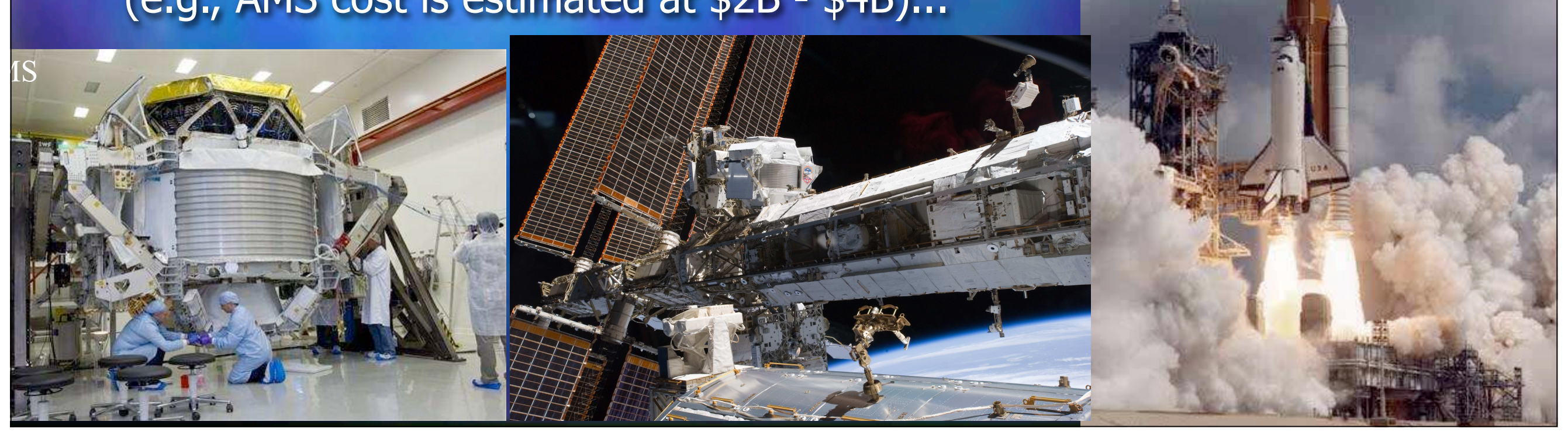




\section{Sone new efforts}
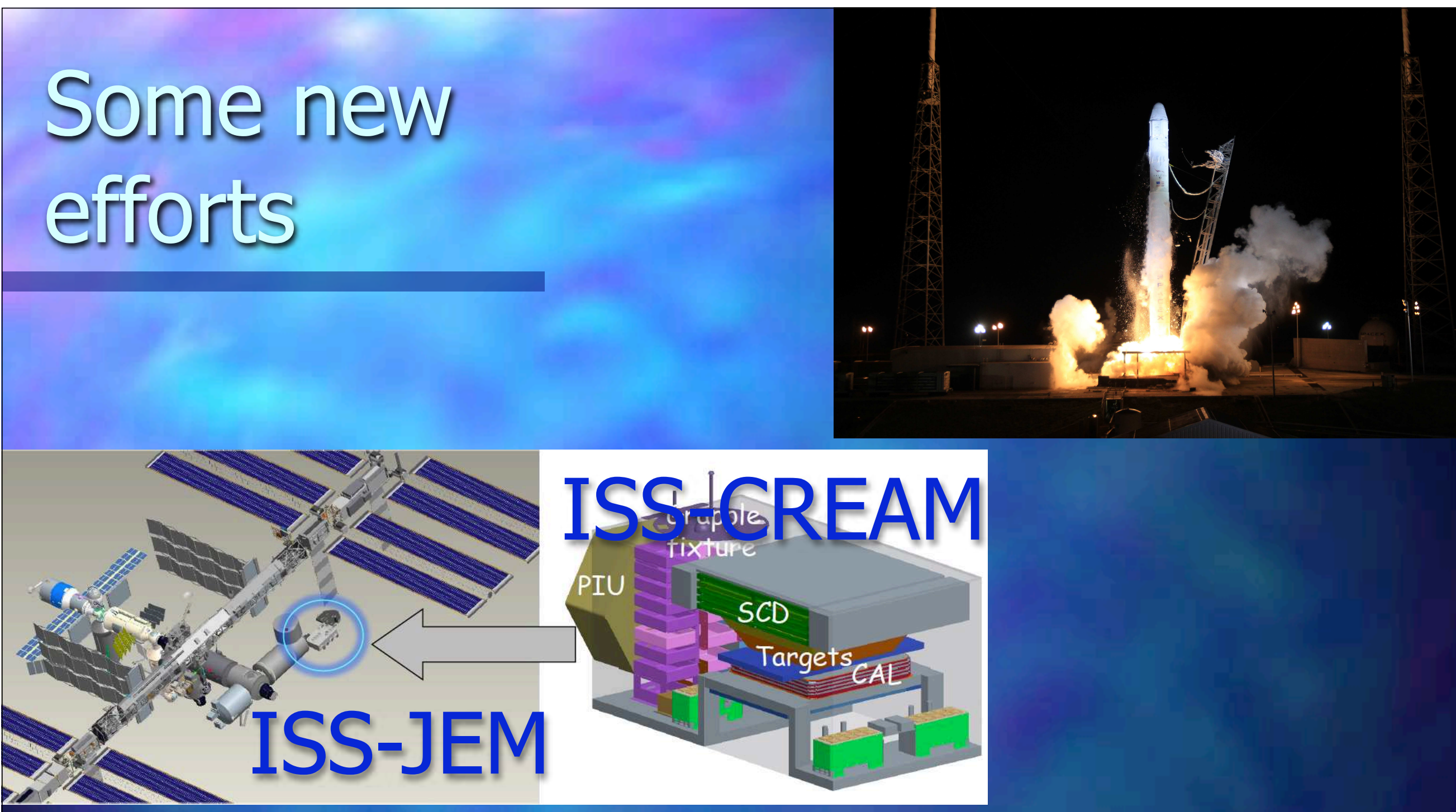

New development: build a scaled-down version of CREAM to go to the ISS

$\Rightarrow$ ISS-CREAM!

optimized for cosmic-ray nuclei, but also sensitive to electrons; long exposure in space will more than compensate for smaller size; in various stages of design, fabrication, qualification, commissioning; planned launch on SpaceX 5, 2014. 
Indjrect measurements Beyond $\sim 10^{14} \mathrm{eV}$, particles become too rare-
for direct detection;

can only be studied through their atmospheric secondaries.

e.g. in the Appenine mountains, Italy:

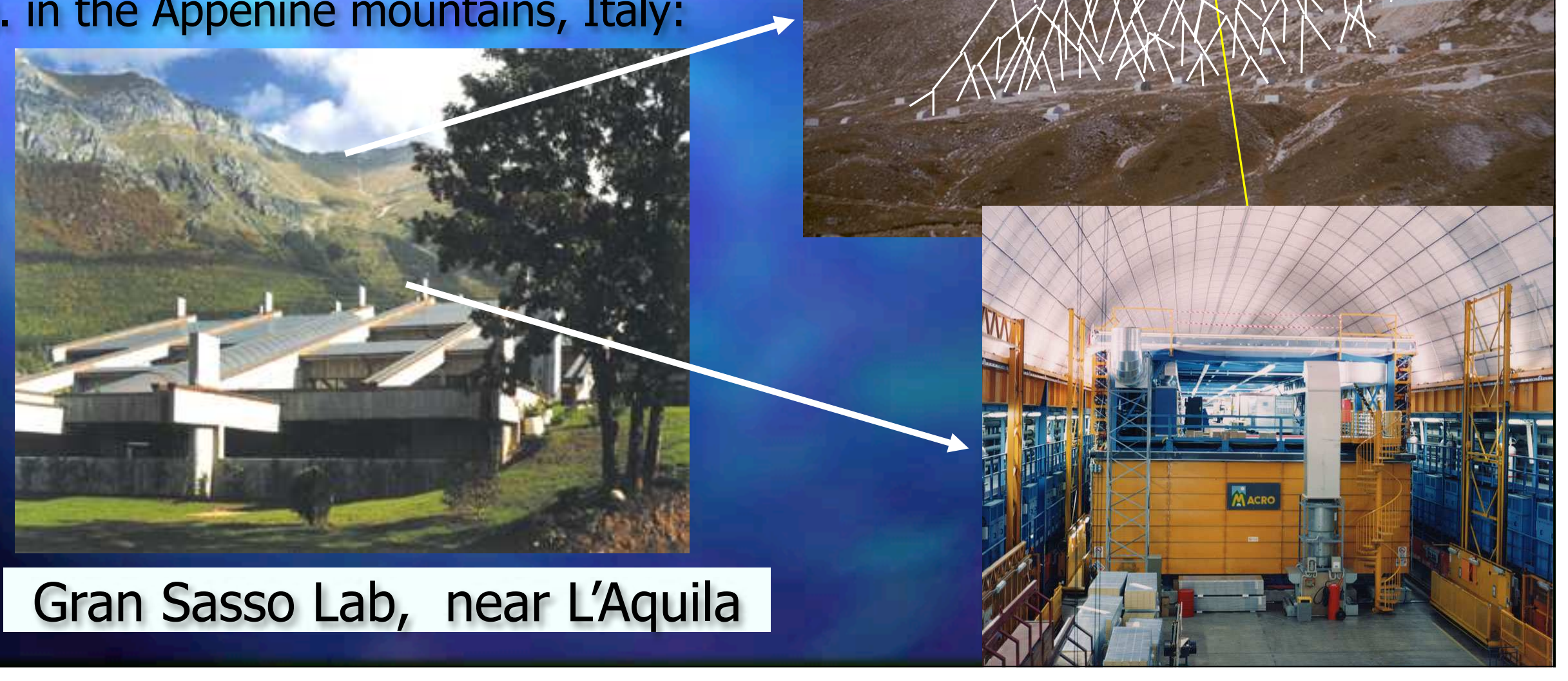




\section{CR air shower}

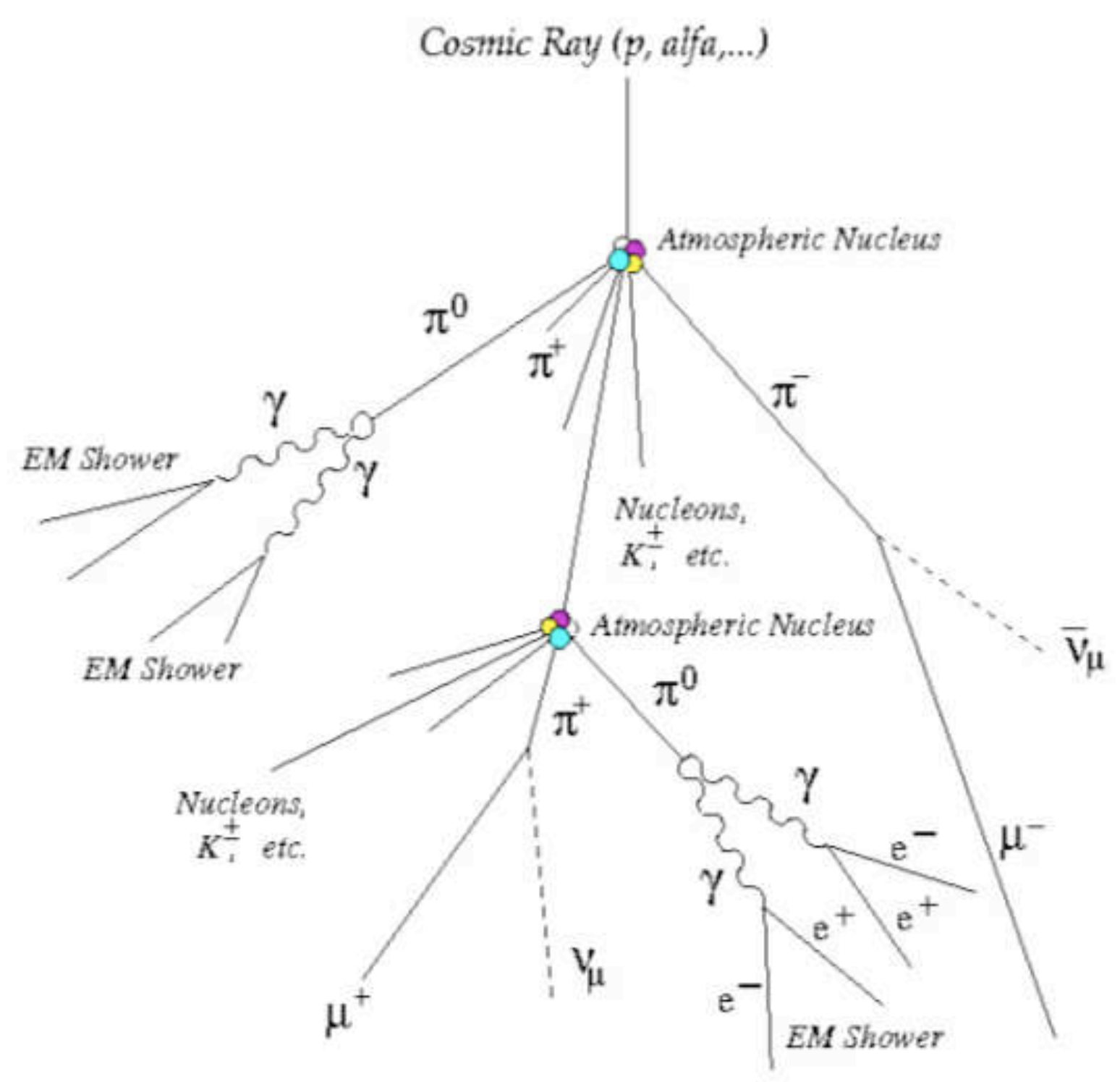

- Primary CR ( $\mathrm{p}, \mathrm{He}, . .$. heavies) interact at top of atmosphere

- Produce cascade of secondary, lighter particles

- Both $\boldsymbol{E M}\left(\mathrm{e}^{ \pm}, \gamma\right)$ and hadronic $\left(\mathrm{N}, \mathrm{K}, \pi, \mu, v_{.}.\right)$ cascades

- Secondaries are detected in air or at ground level 


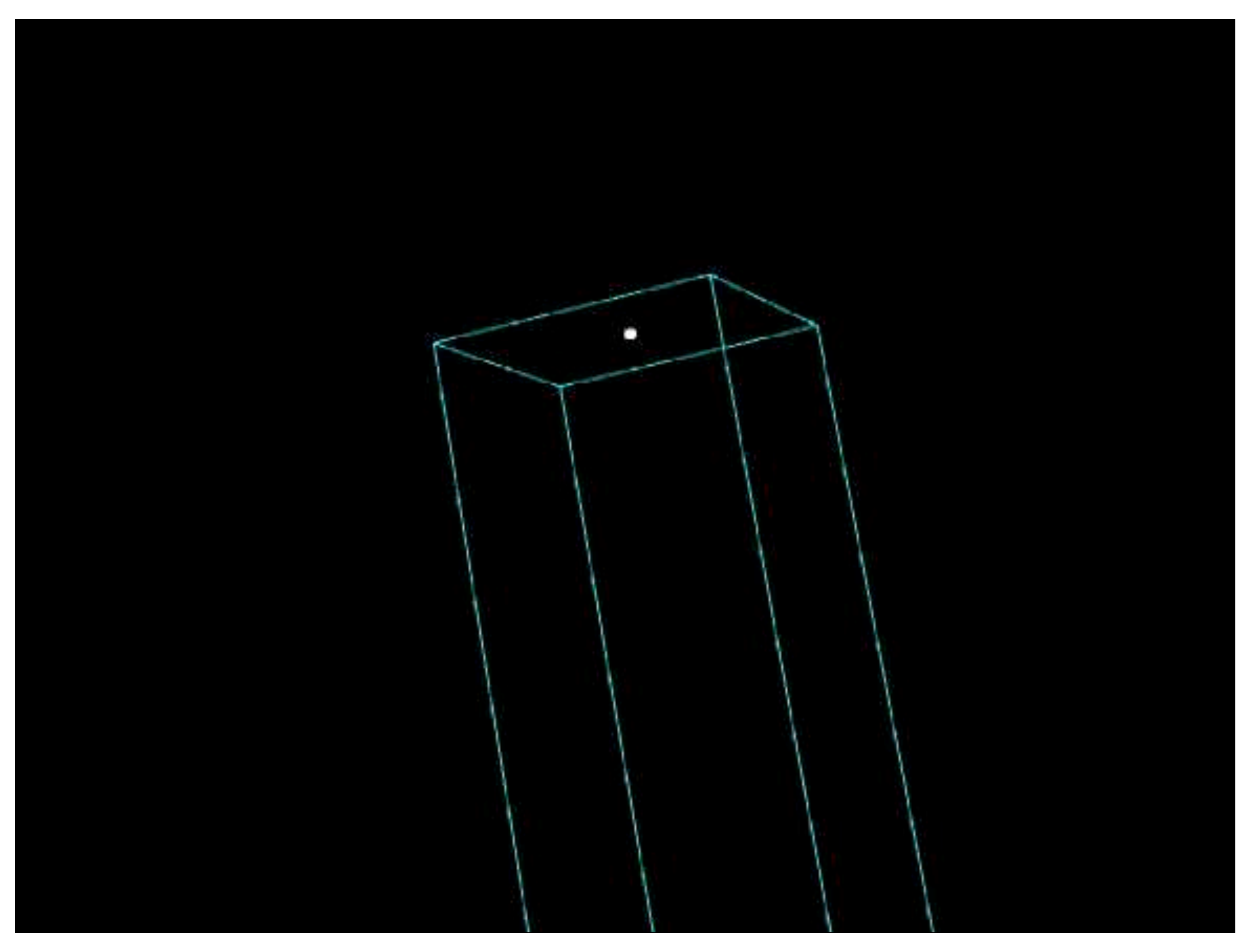




\section{KASCADE-Grande}

KArlsruhe Shower Core Array DEtector - Grande

- Indirect detection of primary CRs $\left(10^{16}-10^{18} \mathrm{eV}\right)$ via secondaries

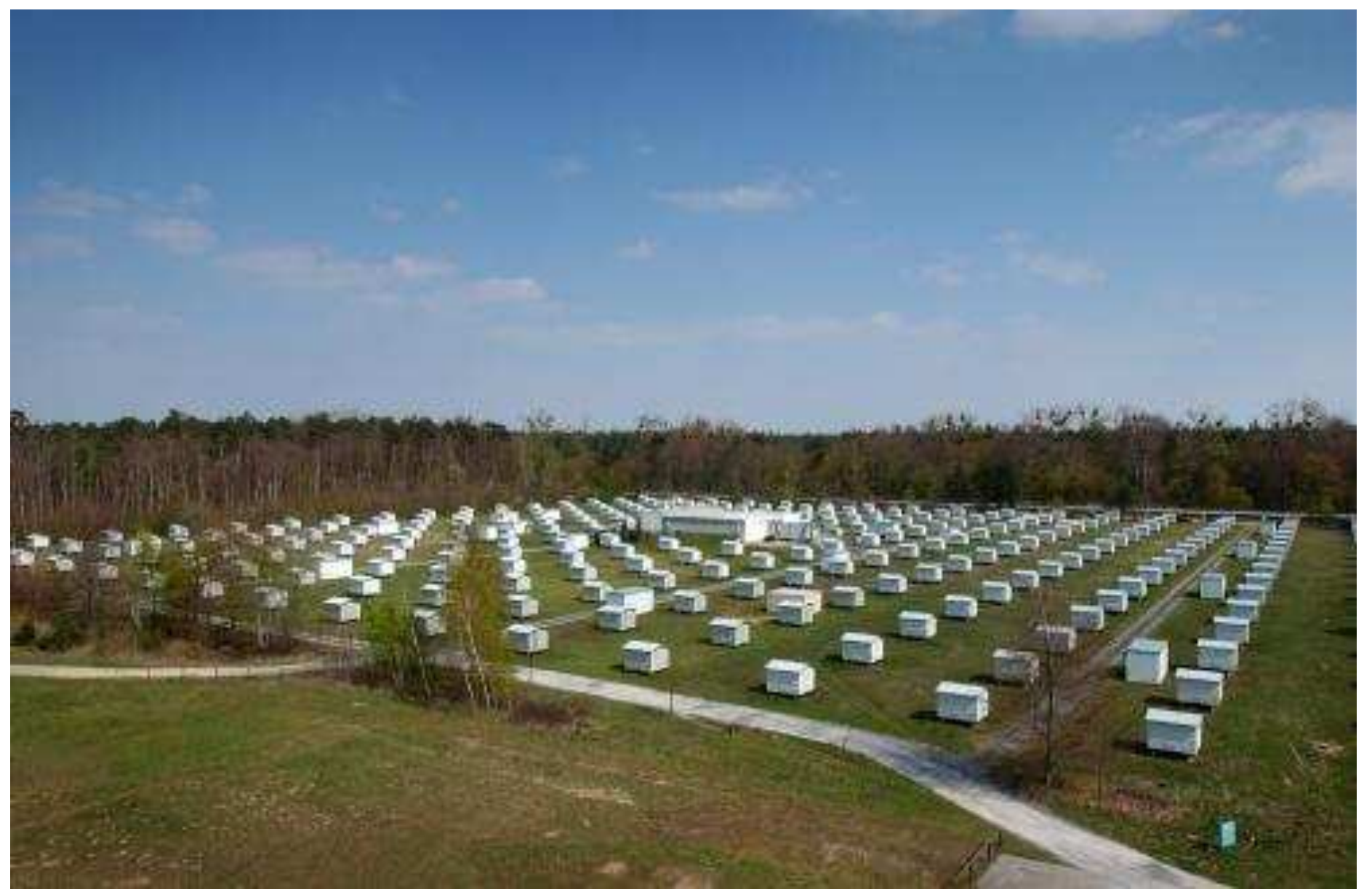

- Monte Carlo simulations allow determination of chemical composition of primary CRs

- Beyond $\mathbf{1 0}^{15} \mathrm{eV}$, composition increasingly weighted towards heavy elements, $\mathrm{He}, . ., \mathrm{C}, \mathrm{O}, . . \mathrm{Fe}$ 


\section{CR spectrum@E $<1^{17} \mathrm{eV}$}

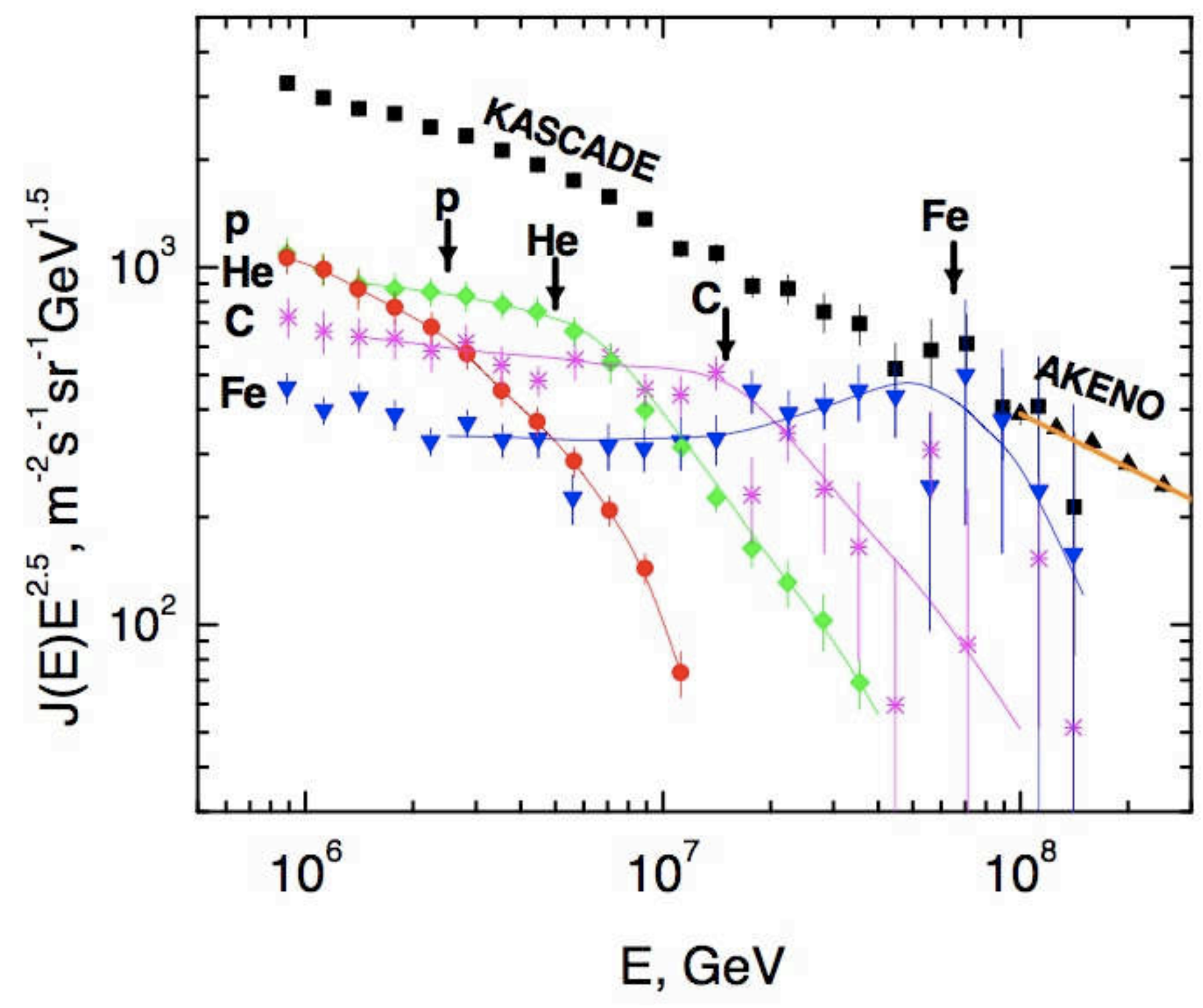

- Spectrum steepens in a "knee"

- Knee energy depends on charge $\mathrm{Z}$

- For $\boldsymbol{p}, \mathrm{knee}$ (a) $10^{15} \mathrm{eV}$

- For $\boldsymbol{F e}$, knee (a) $10^{17} \mathrm{eV}$

$\mathrm{E}_{\max } \sim \beta \mathrm{cZeBL} \boldsymbol{\mathcal { }}$ 


\section{Push to even high energies}

At the highest energies, extraordinary efforts are required to measure the air showers with enough statistical precision:

Volcano Ranch 1959-1965, $100 \mathrm{~km}^{2} \mathrm{yr}$ total; Haverah Park 1963-1987, $300 \mathrm{~km}^{2} \mathrm{yr}$ total;

Yakutsk 1973-2000, $500 \mathrm{~km}^{2} \mathrm{yr}$ total; Fly's Eye 1987-1995, $900 \mathrm{~km}^{2} \mathrm{yr}$ total; AGASA $1991-2003,3,000 \mathrm{~km}^{2} \mathrm{yr}$ total; HiRes 1998-2006, $8,000 \mathrm{~km}^{2} \mathrm{yr}$ total; Auger since $2003,21,000 \mathrm{~km}^{2} \mathrm{yr}$ so far; TA since $2008,700 \mathrm{~km}^{2} \times \varepsilon \mathrm{T}$ so far.

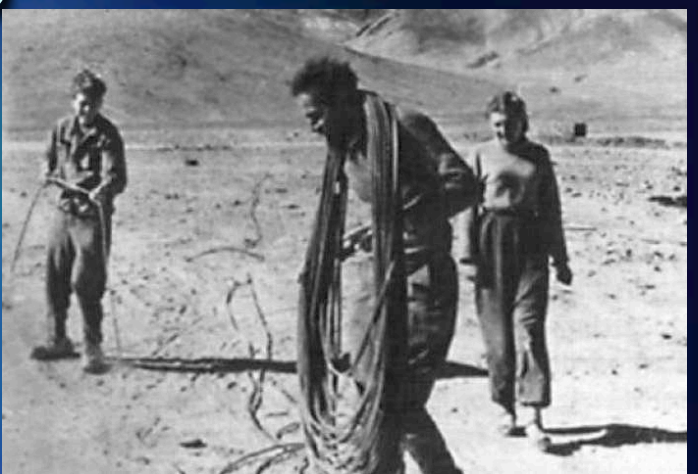

G. Zatsepin in R

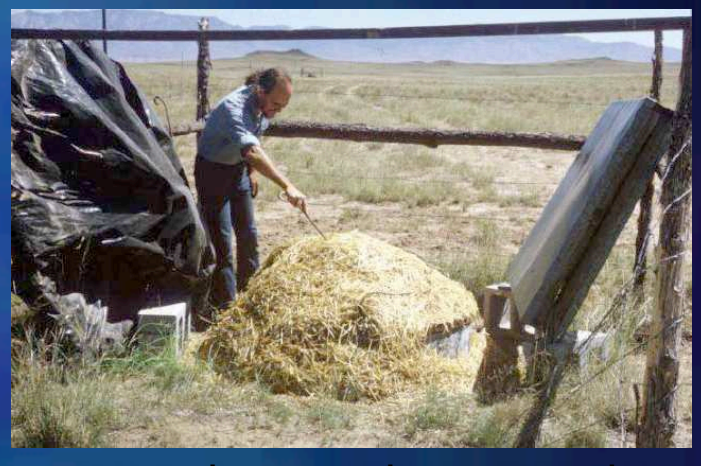

J. Linsley at Volcano Ranc
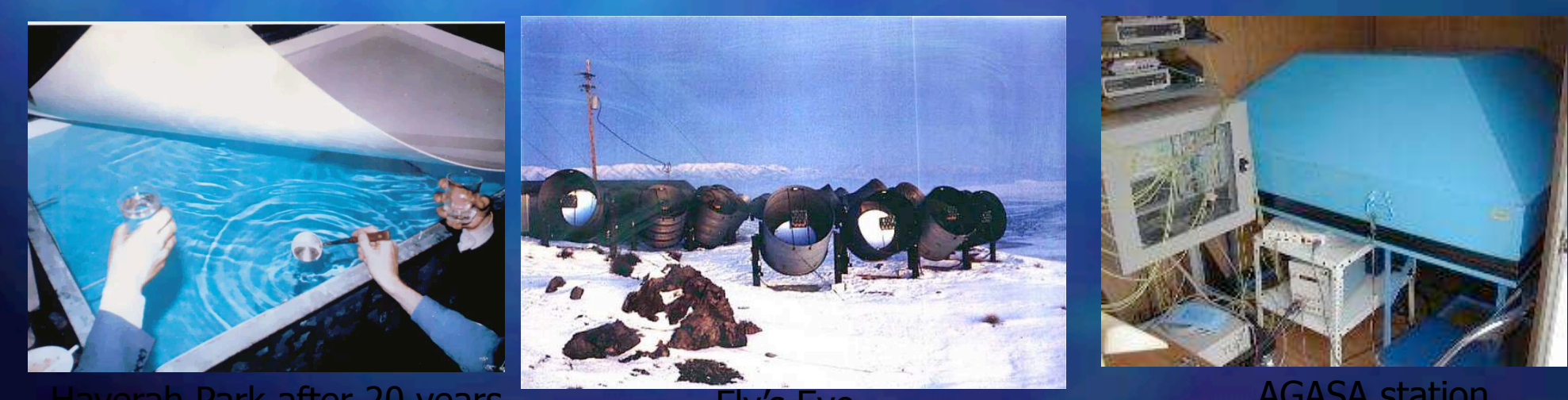

Fly's Eye 


\section{currently,}

highest energy UHECR observatory with largest daily rate

$$
\text { is ... }
$$




\section{Pierre Auger Observatory}

International consortium, located in Argentina, Mendoza province

\section{Uses two techniques} for detecting CR shower:

- detect air fluorescence produced by shower particles (FD)

(EM showers)

- detect shower particles on the surface (SD) (hadronic showers)

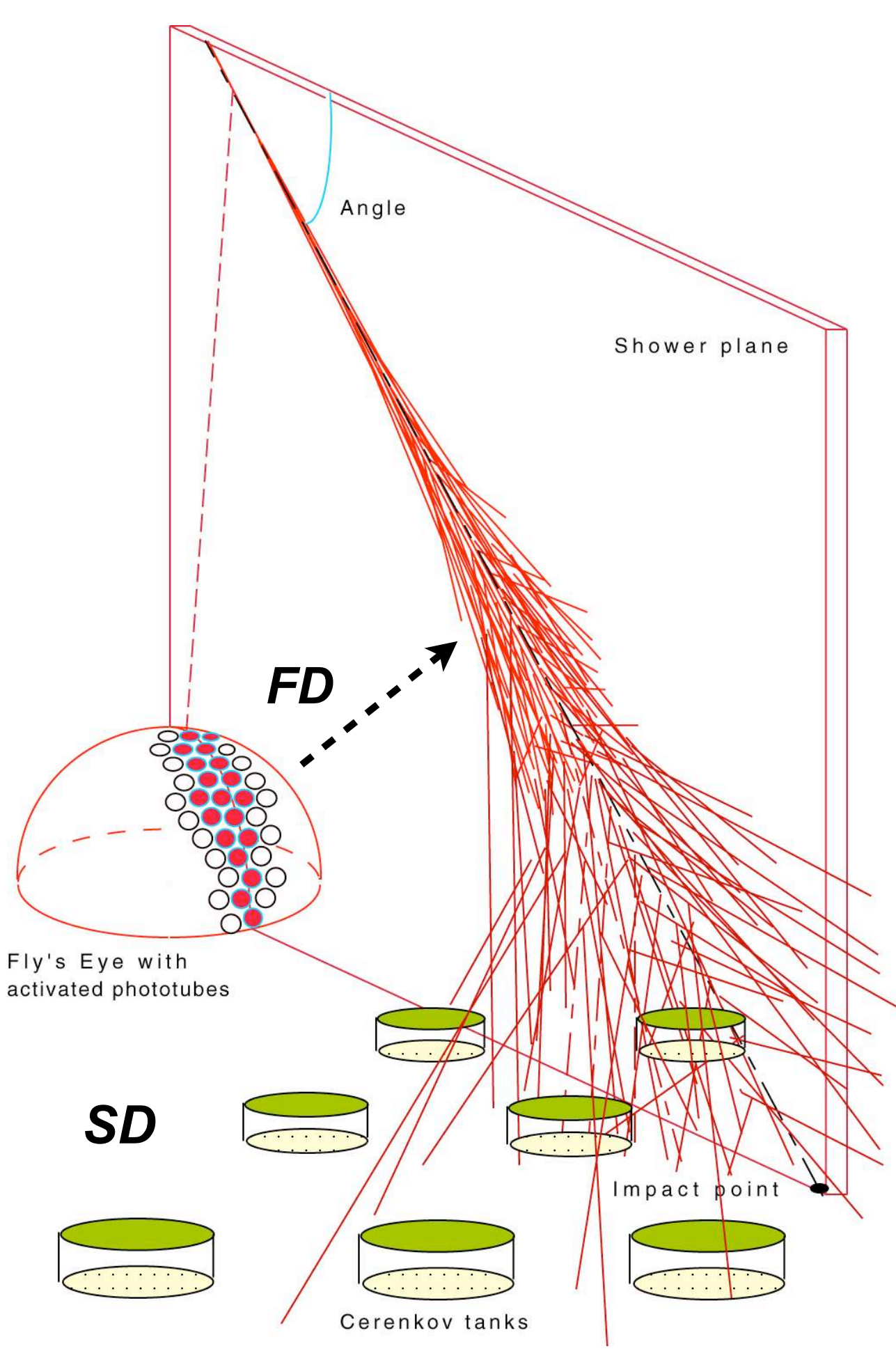




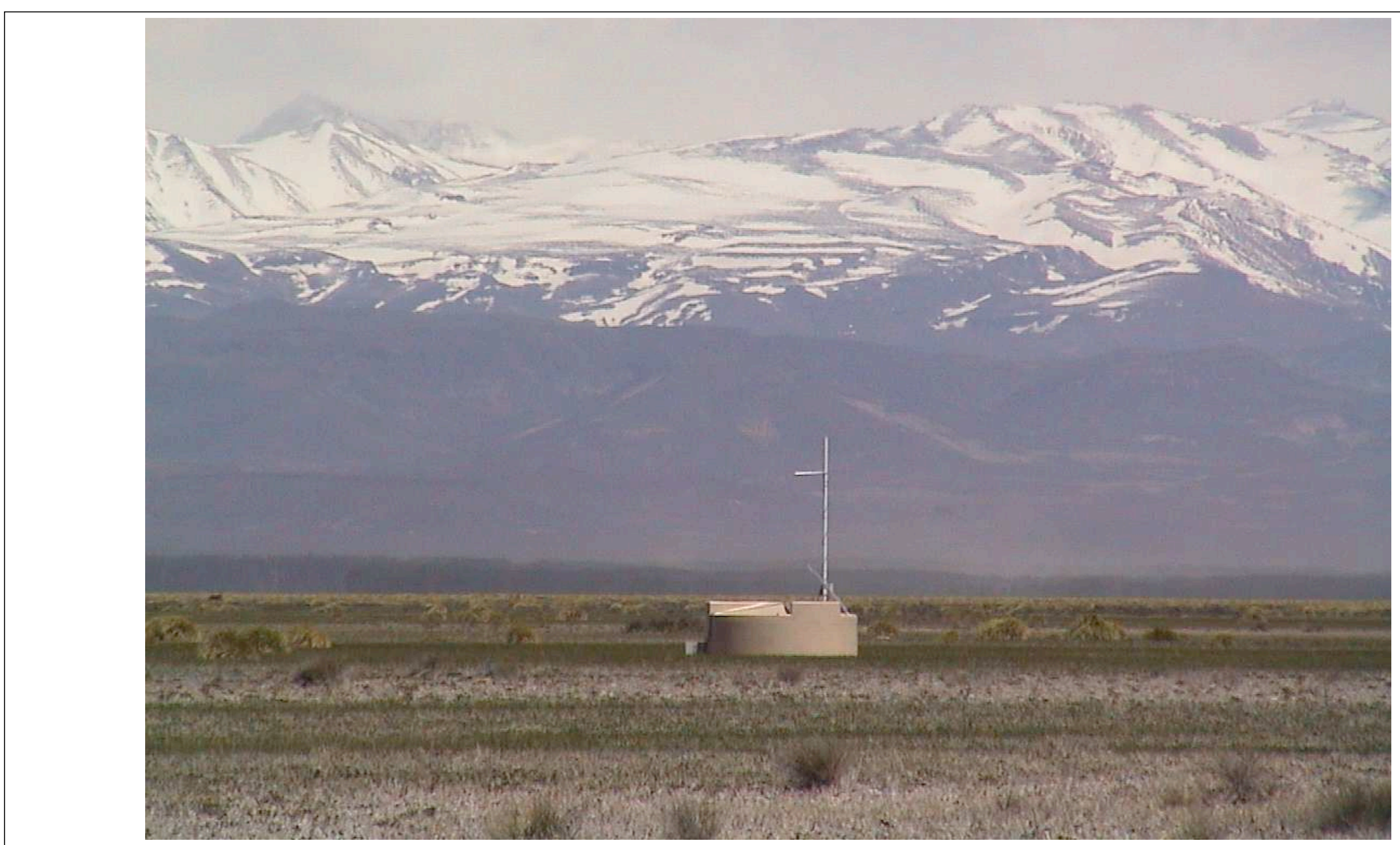

Surface detectors (SD) Muons from shower $\rightarrow$ Cherenkov light in water tank, detected by phototubes

Pierre Auger Observatory: Malargue, Mendoza, Argentina: $E \sim \mathbf{1 0}^{17}-10^{21} \mathrm{eV}$ -1600 surface detectors: water Cherenkov tanks, 11 kliters ea., $1.5 \mathrm{~km}$ apart -32 air fluoresence telescopes, $4 \times 8$ arrays of 30x30 deg. sky coverage 29 -Also: tau-nu (horiz.l shower capability: Earth-skimming \& through Andes) 


\section{Fluorescence detector (FD)}

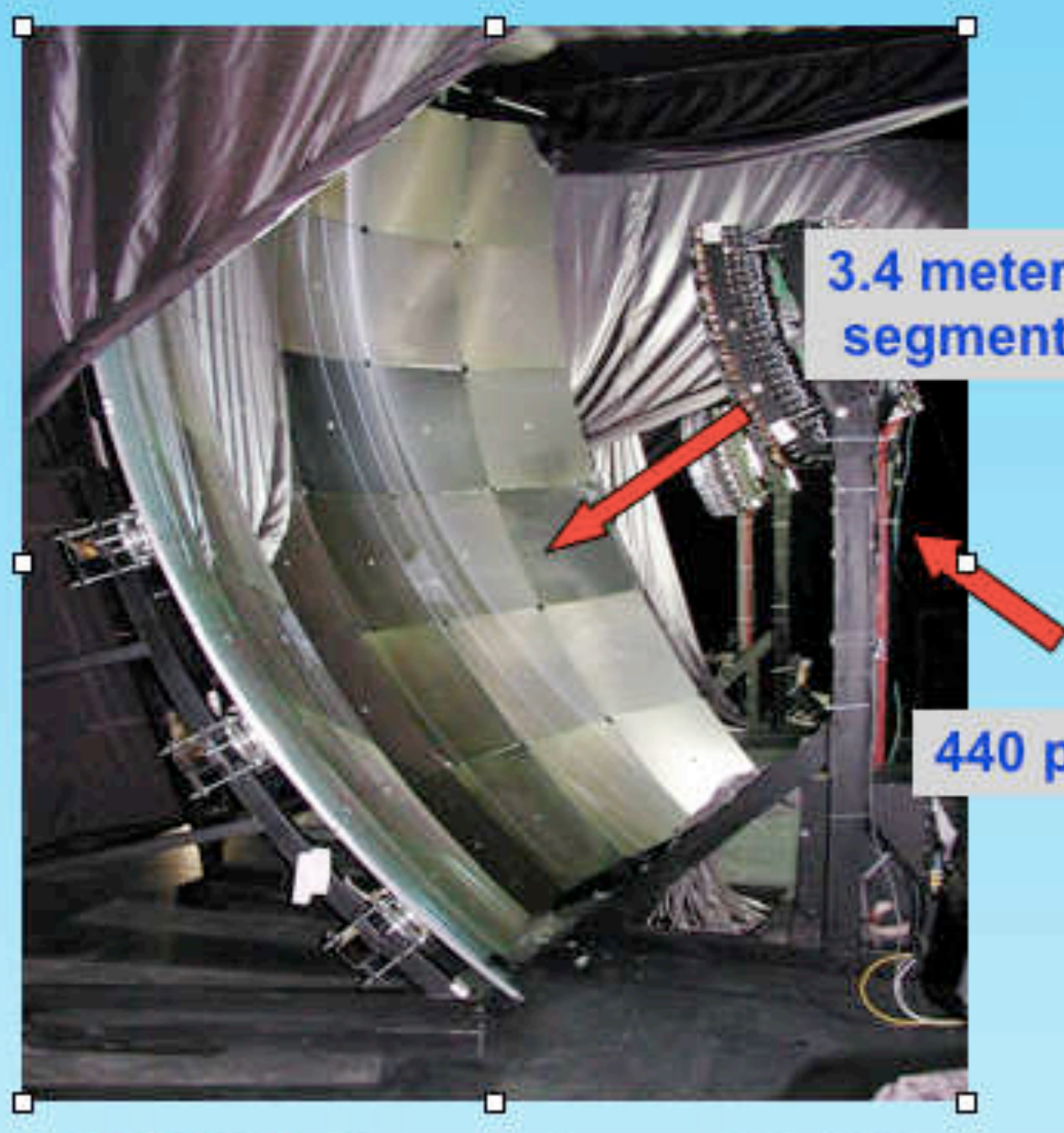

EM shower produces e \pm pairs, which impact on $\mathrm{N}_{2}$ molecules $\rightarrow$ fluorescence light observed by FD

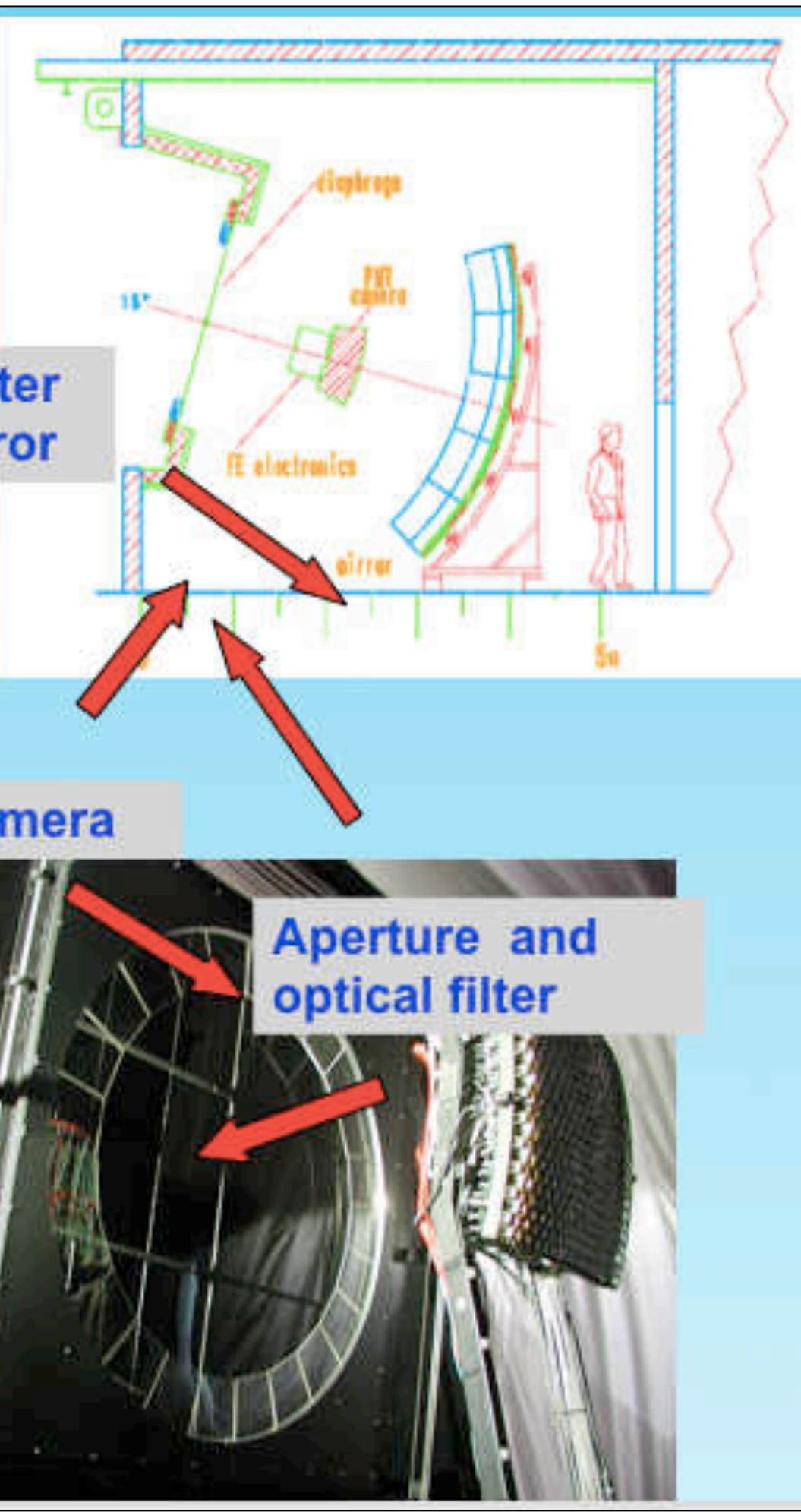




\section{Auger Obs. - 3000 km² uhecr detector \\ Mendoza, Argentina}

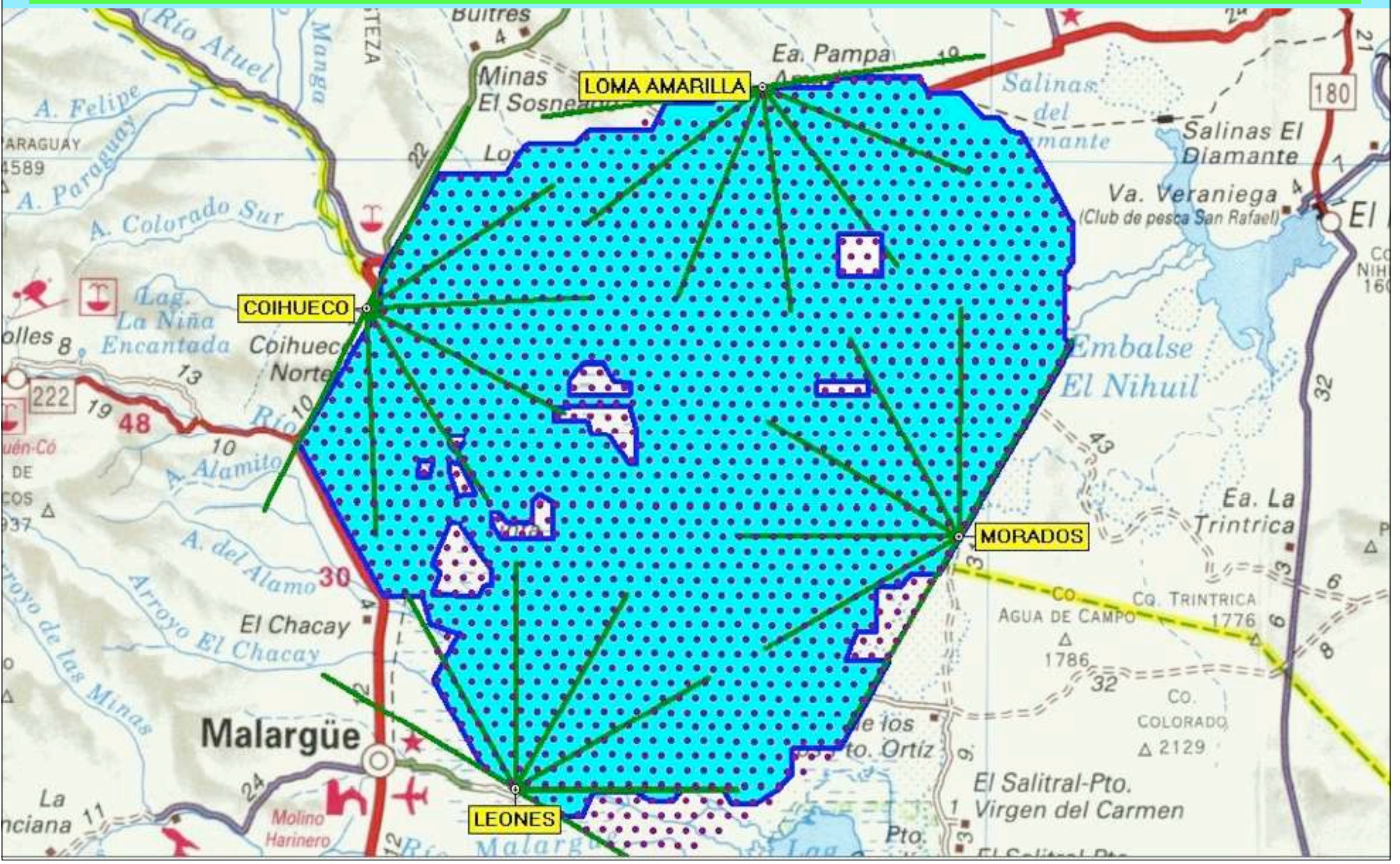




\section{Size of the Pierre Auger Observatory (vs Budapest)}

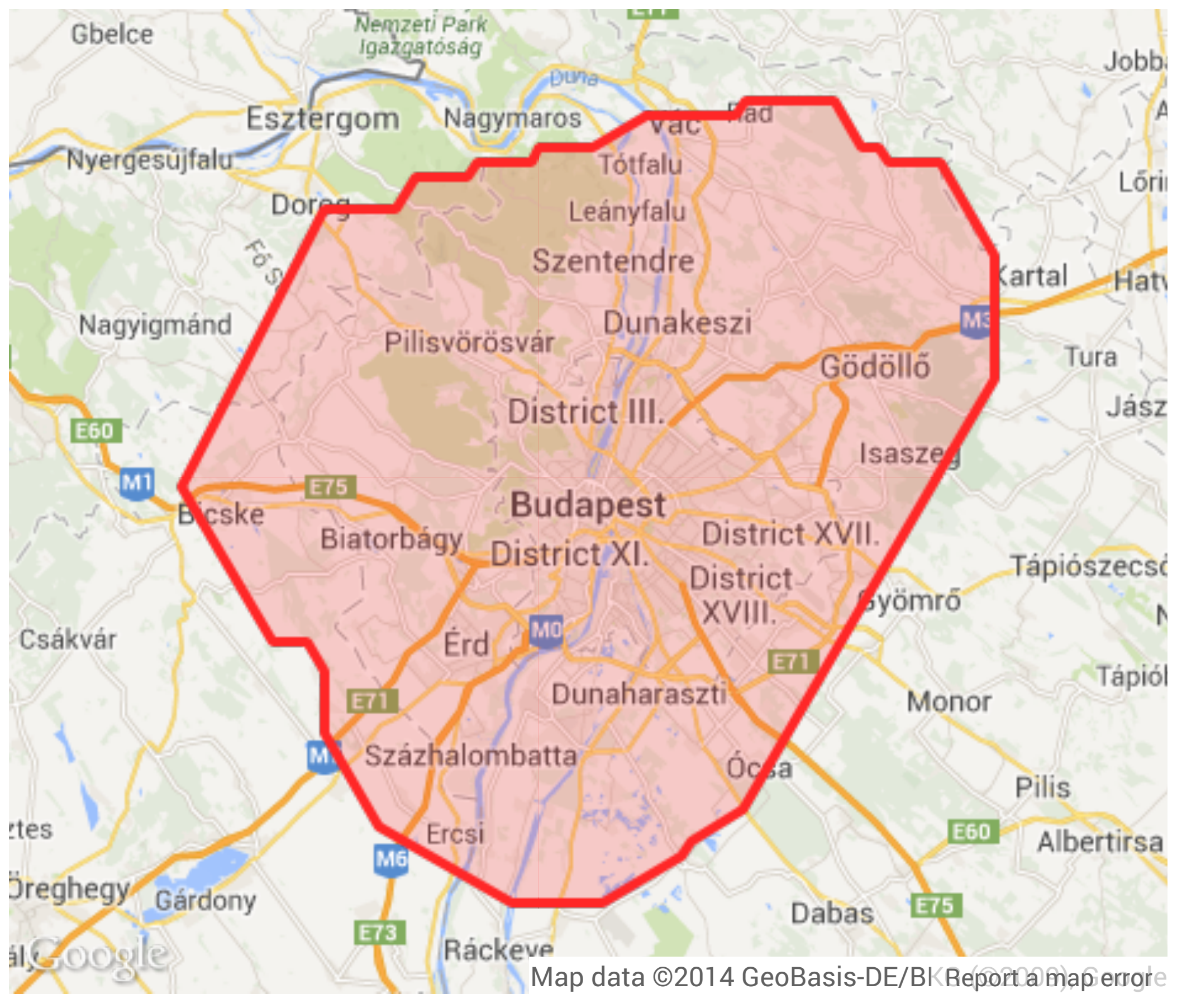




\section{The Pierre Auger Observatory}

(movie credit: Auger collab.)

Jim Cronin

Alan Watson

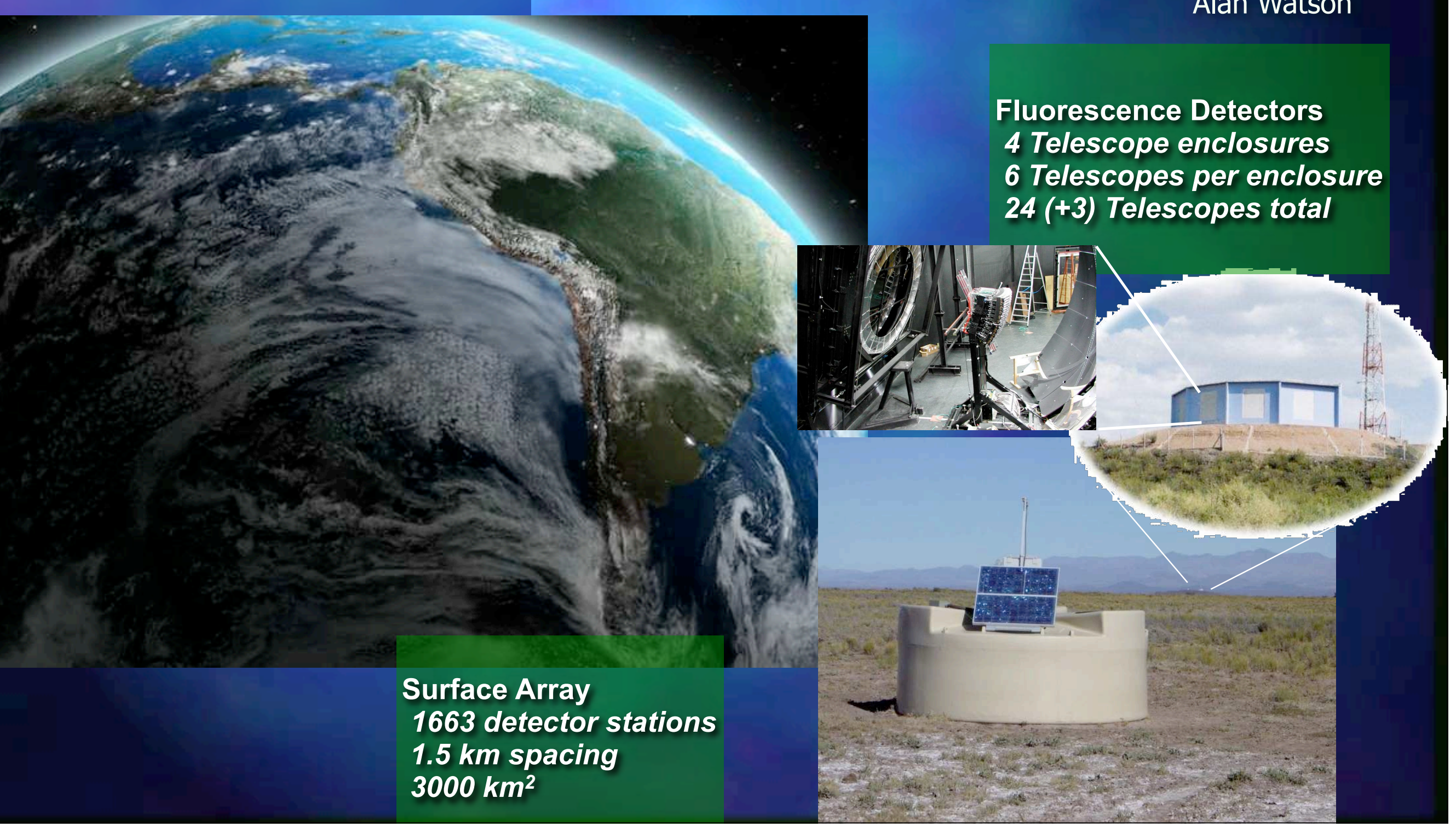




\section{The cosmic ray spectrum}

\section{Cosmic rays:}

- high energy nuclei from $\mathrm{H}$ to $\mathrm{Fe}$; $\sim<10^{9} \mathrm{eV}$ to $>10^{20} \mathrm{eV}$; rates plummet with energy...

11 orders of magnitude in energy; 31 orders of magnitude in intensity...

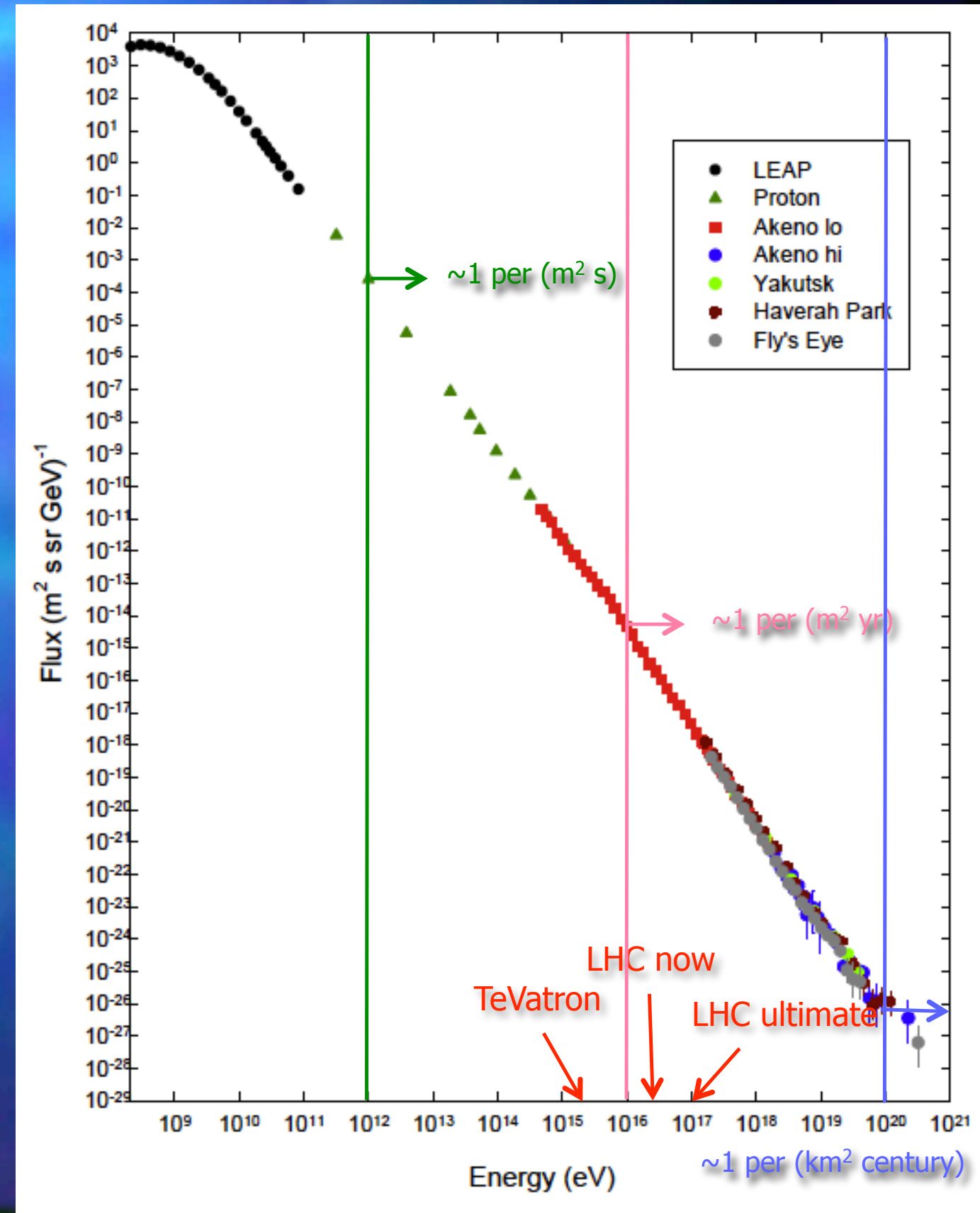




\section{The cosmic ray spectrum}

\section{Cosmic rays:}

- $\quad$ high energy nuclei from $\mathrm{H}$ to $\mathrm{Fe}$; $\sim<10^{9} \mathrm{eV}$ to $>10^{20} \mathrm{eV}$; rates plummet with energy...

11 orders of magnitude in energy; 31 orders of magnitude in intensity...

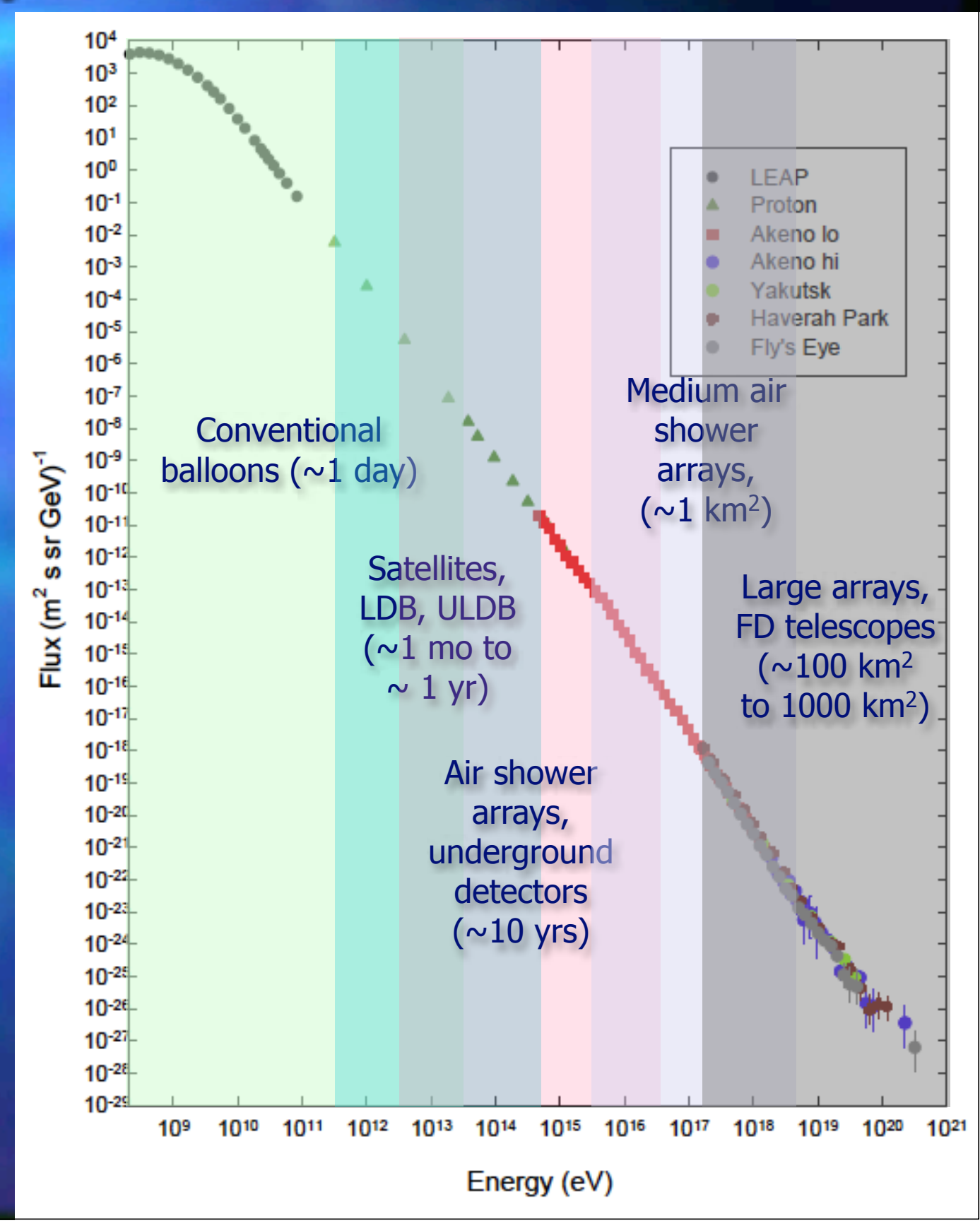




\section{The cosmic ray spectrum}

\section{Cosmic rays:}

- $\quad$ high energy nuclei from $\mathrm{H}$ to Fe; $<10^{9} \mathrm{eV}$ to $>10^{20} \mathrm{eV}$; rates plummet with energy...

11 orders of magnitude in energy; 31 orders of magnitude in intensity.

\section{Fluxes rescaled by $E^{2}$}

The Knee:

Limit to supernova acceleration?

Clue: composition

The Ankle:

Transition to extragalactic sources?

Clue: anisotropies, energy cutoff

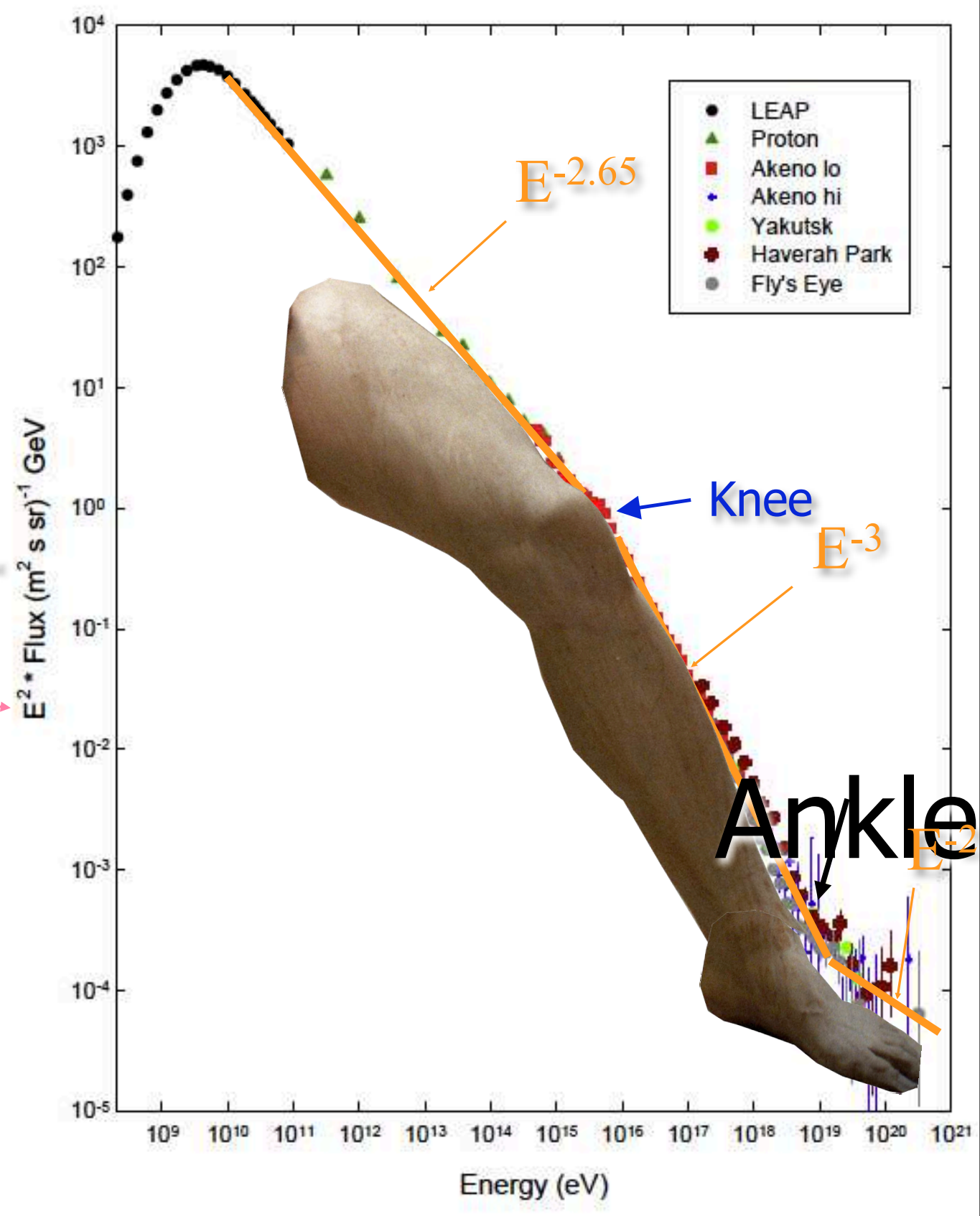




\section{Cosmic ray flux and Composition}

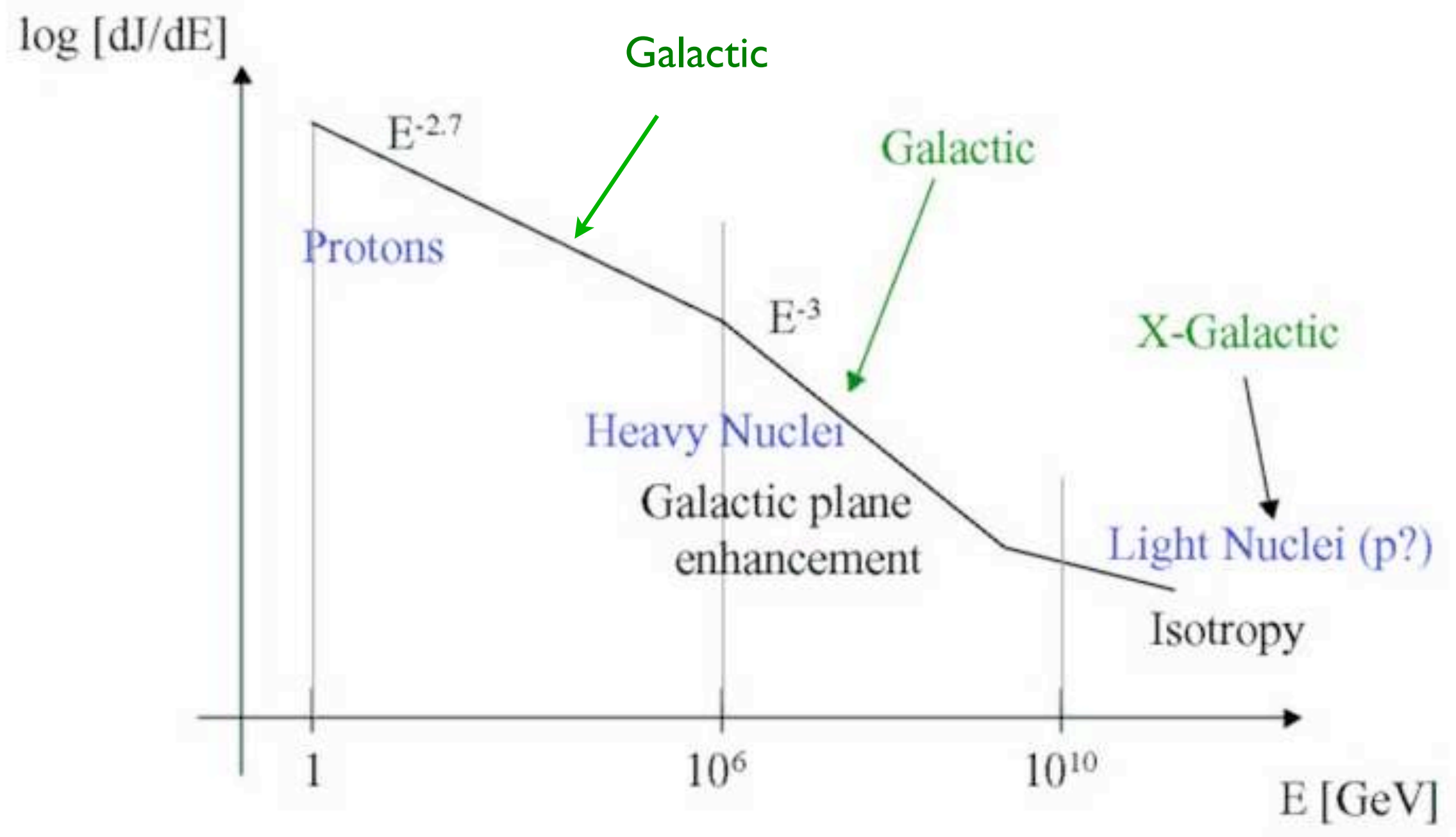

$U_{c r}(1 \mathrm{GeV})=1 \mathrm{eV} / \mathrm{cm}^{3}$ 


\section{UHE spectjum}

\section{Auger 2011}

63,376 SD + 3,660 hybrid events;

Exposure $=20,905 \mathrm{~km}^{2} \mathrm{sr} \mathrm{yr}$

(7×AGASA, 2.6 $\times$ HiRes).

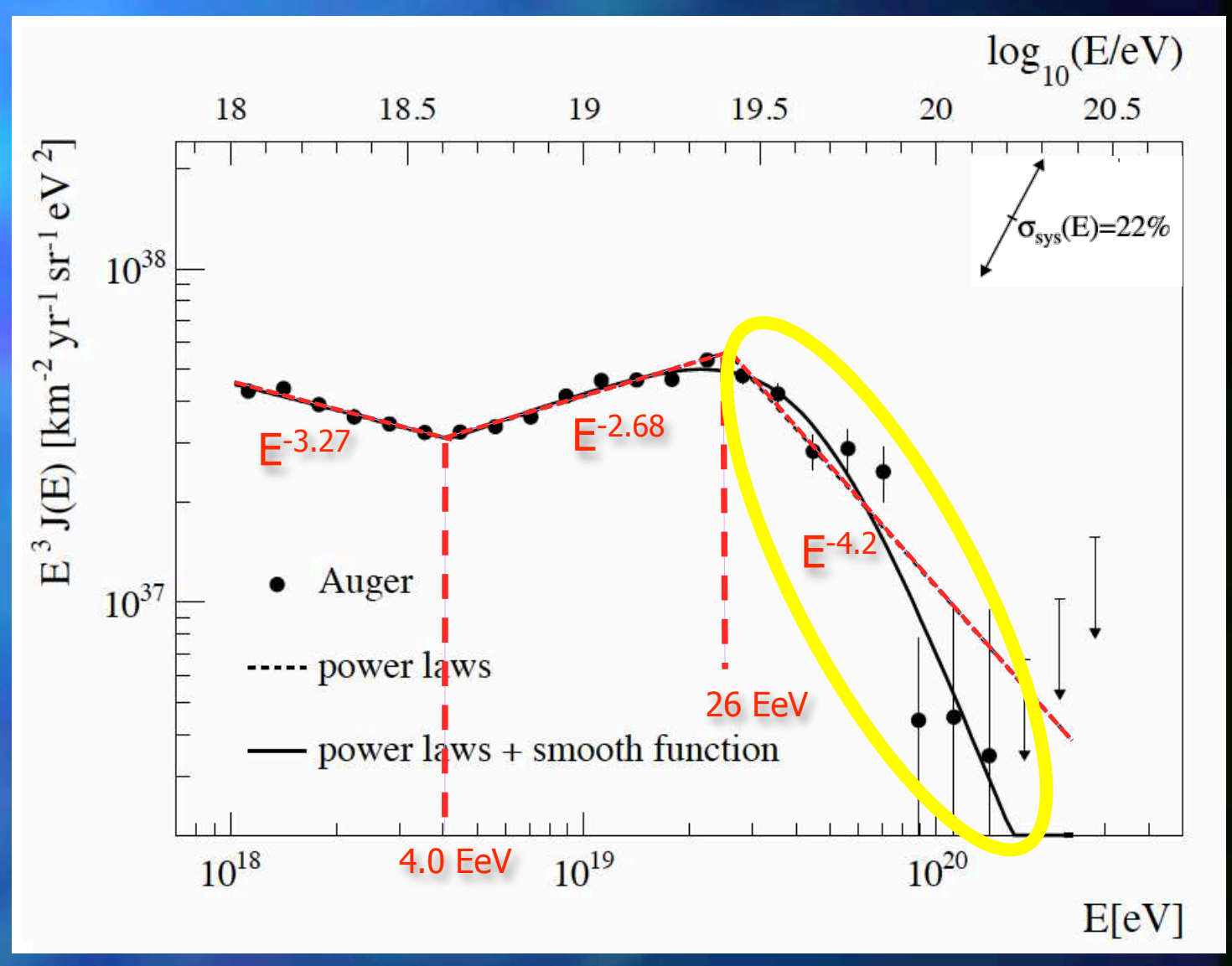

GZK cutoff (or something very like it) definitely seen!

Sources must be within 100 Mpc (300 Mly)... look for anisotropies! 38 


\section{How far do they come from?}

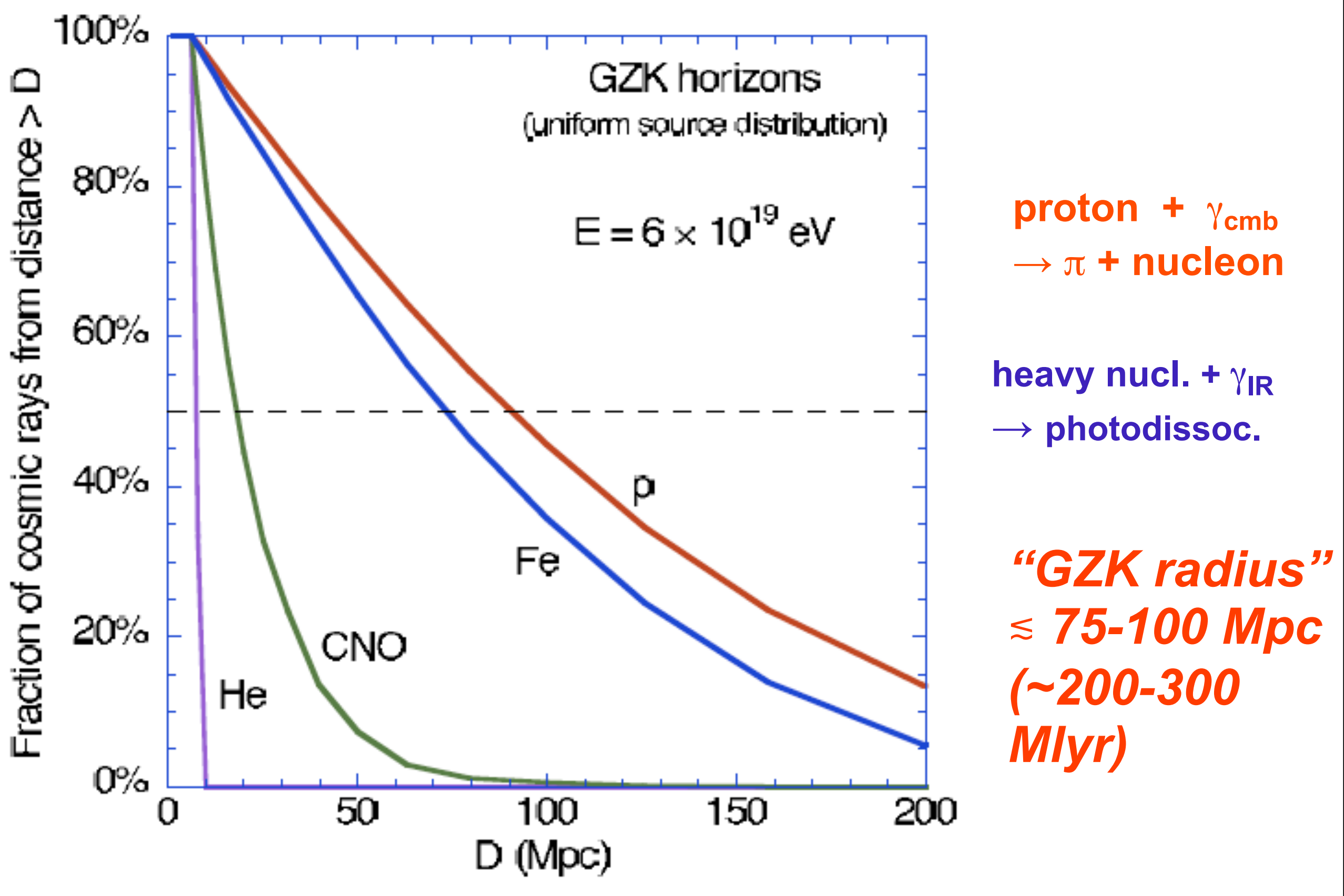




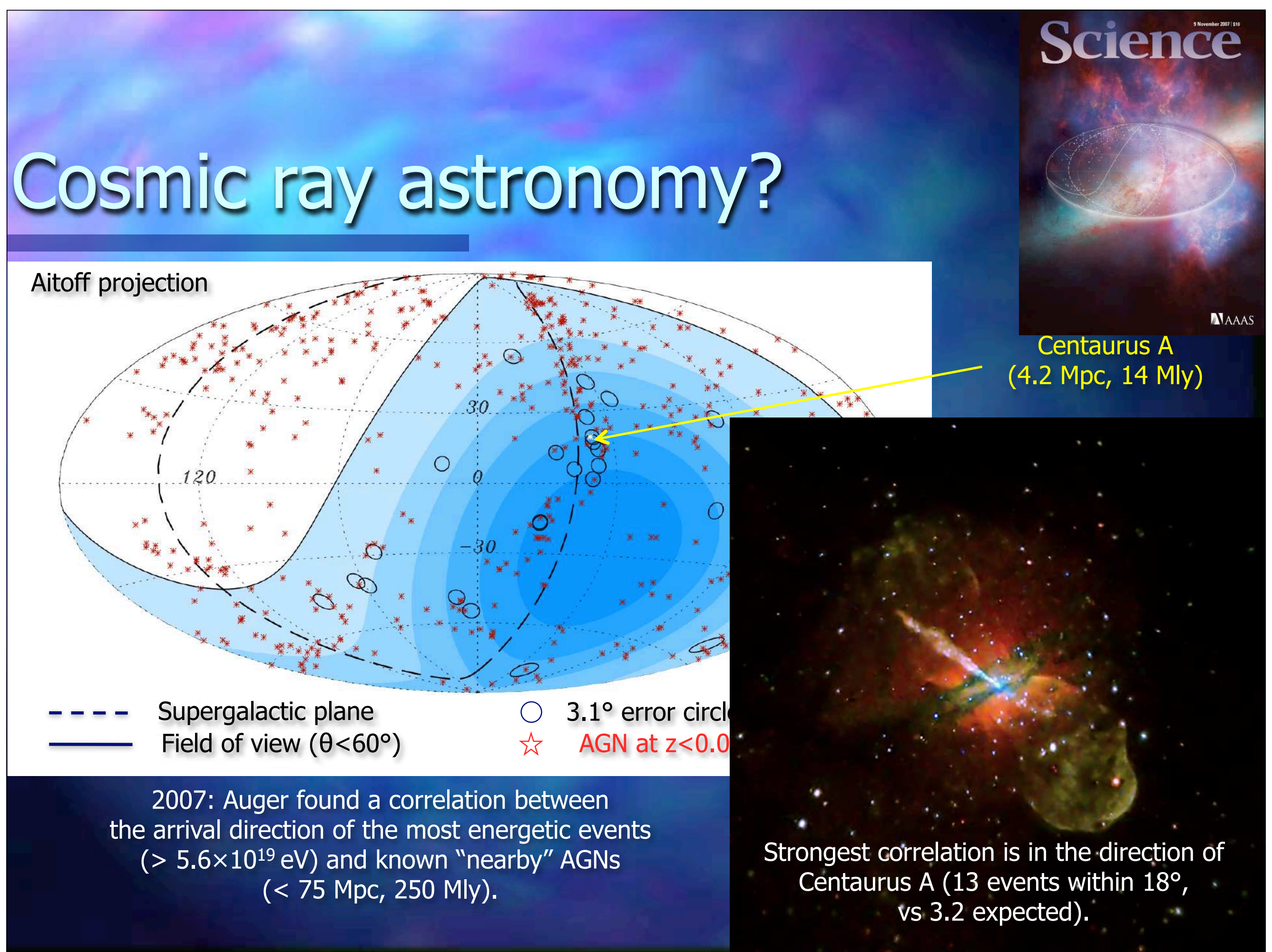




\section{AUGER :}

\section{UHECR spatial correlations with AGNs (or LSS)}

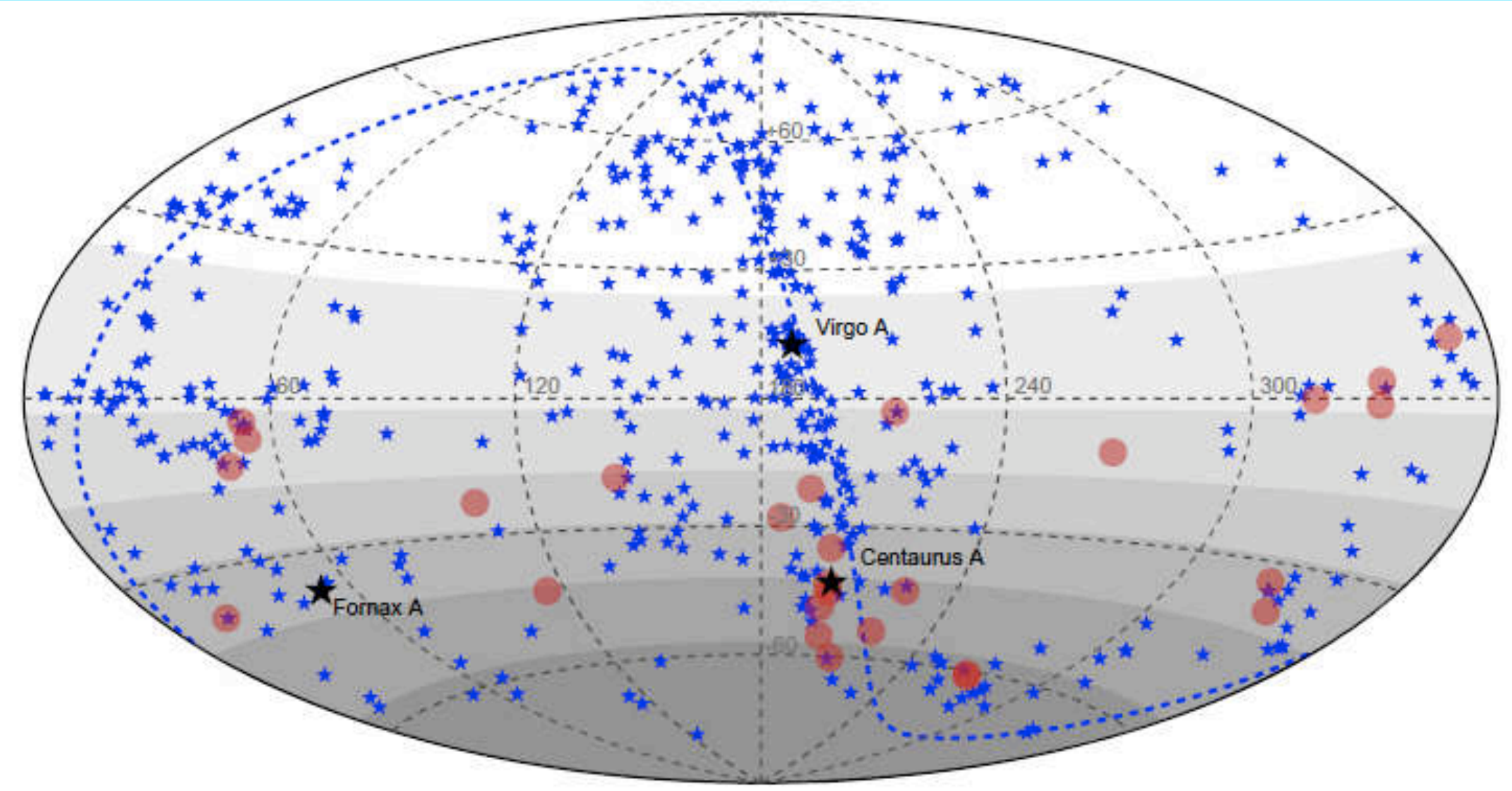

- Dashed line: supergalactic equator

- Circles (proton): Events E $>5 \times 10^{19} \mathrm{eV}, \mathrm{D}<75 \mathrm{Mpc}$

- Asterisks : Veron-Cety catalog AGNs 


\section{Auger spatial correlation}

- Initially found 3- $\sigma$ corr. with VC AGNs within $\theta \leq 3.5^{\circ}$ and $\mathrm{D}<$ $75 \mathrm{Mpc}$, for 27 events $\mathrm{E}>4.5 \times 10^{19} \mathrm{eV}$ (Science, 2007)

- The above correlation would suggest protons

- But: there is even better correlation with "average" galaxies

- If heavy: $r_{L}$ smaller, rms. dev. angle $\theta \sim n^{1 / 2} \theta_{s} \sim\left(r / \lambda_{B}\right)^{1 / 2}\left(\lambda_{B} / r_{L}\right) \sim$ $\left(\mathrm{r} \lambda_{\mathrm{B}}\right)^{1 / 2} / \mathrm{r}_{\mathrm{L}}$ is larger, many more gals. inside error circle

- Also: (arXiv:1009.1855, etc. ): now (>2011) the VCV-AGN significance has weakened to $\leqslant 2 \sigma$ (see Allard talk)

- Low or no VCV AGN corr.: also from HiRes (Sagawa talk)

$\rightarrow$ Could be sources are in galaxies - GRB ? HNs? MGRs? Or in other, less extreme and more common galaxies?

$\rightarrow$ Or could be they are heavy nuclei, larger error cirele? 


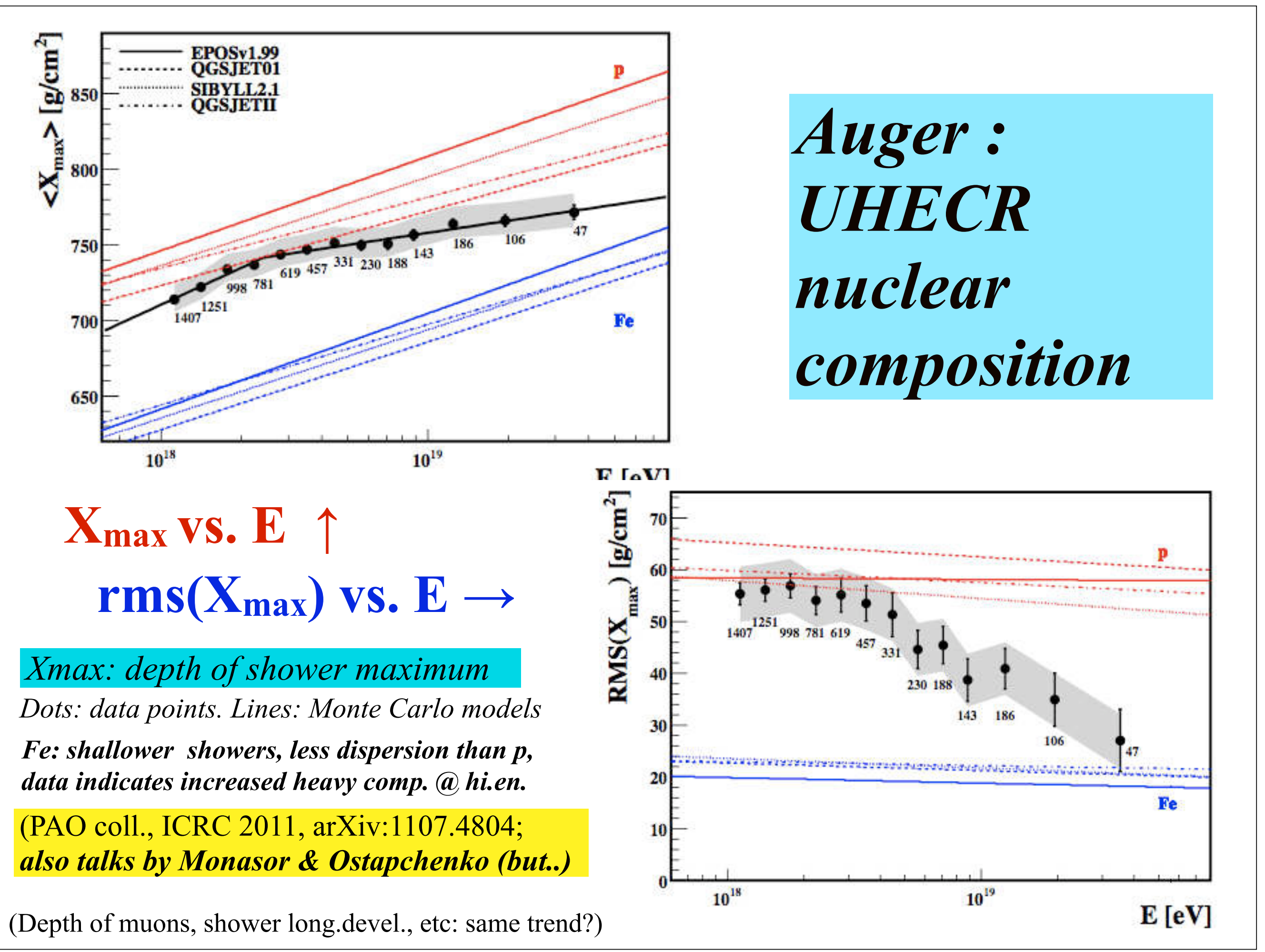




\section{GZK energy}

- "GZK"= Greisen-Zatsepin-Kuzmin (1967)

- "UHECR" = ultra-high energy cosmic ray, roughly $10^{18}-10^{21} \mathrm{eV}=\mathbf{1 0}^{-2}-10 \boldsymbol{E}_{\boldsymbol{G Z K}}$

- $E_{G Z K} \sim 10^{20} \mathrm{eV} \equiv 100 \mathrm{EeV}$ (Exa-electron-Volt) $\approx 1.6 \times 10^{8} \mathrm{erg} \approx 16$ Joule $\approx 4$ calories

- $\boldsymbol{E}_{\boldsymbol{G Z K}} \approx$ fast-serve tennis ball $(\sim 130 \mathrm{~km} / \mathrm{h})$, or $\sim$ the energy of a small caliber pistol bullet

- Significance: $E \gtrsim E_{G K K}$ protons encountering the $\sim 10^{-3} \mathrm{eV}$ cosmic microwave (CMB) photons undergo catastrophic photo-hadronic losses, $p+\gamma \rightarrow \pi+N \rightarrow v, \gamma, e^{+}$ 
Other (northern) UHECR experiment:

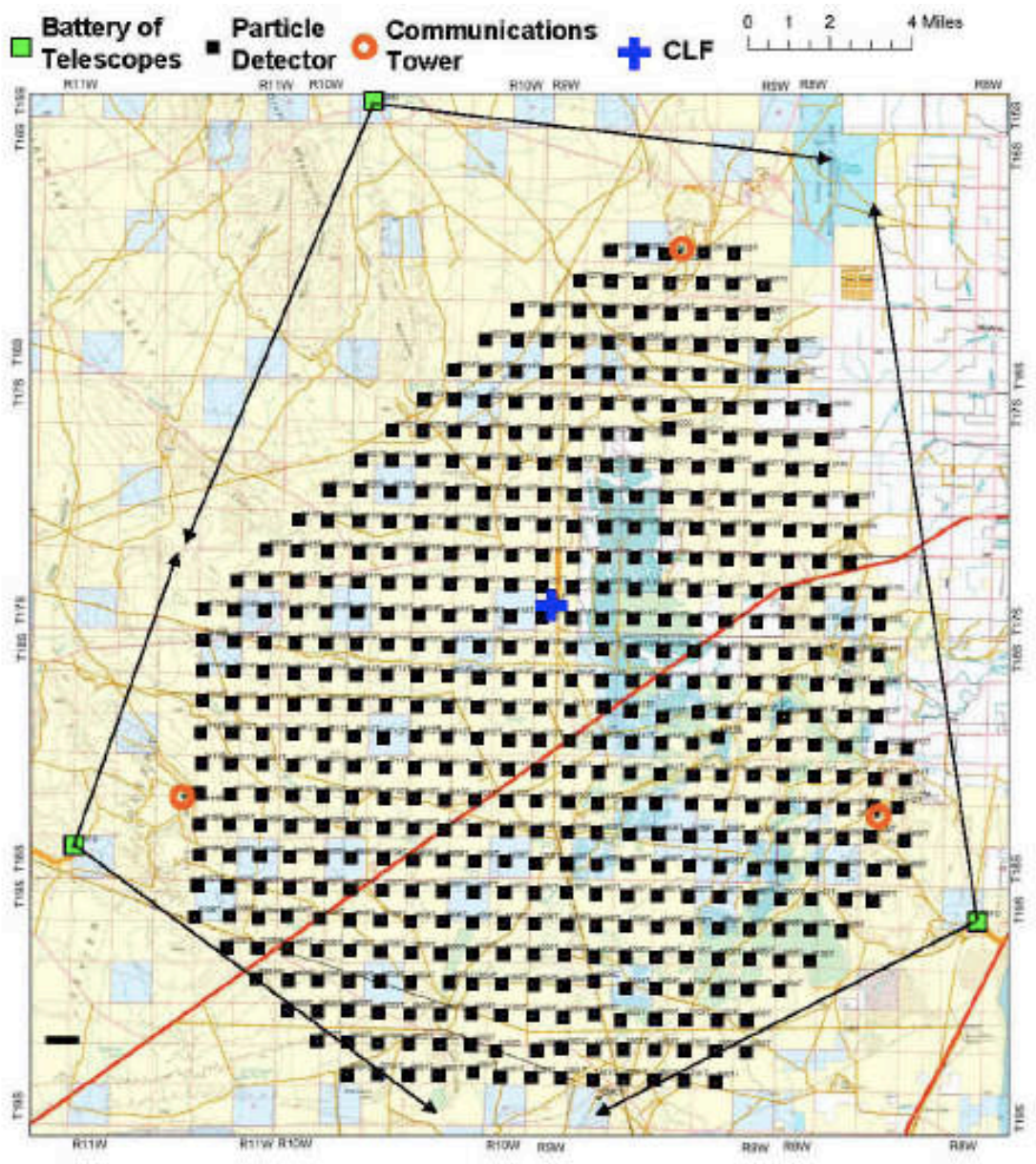

\section{Array (TA)}

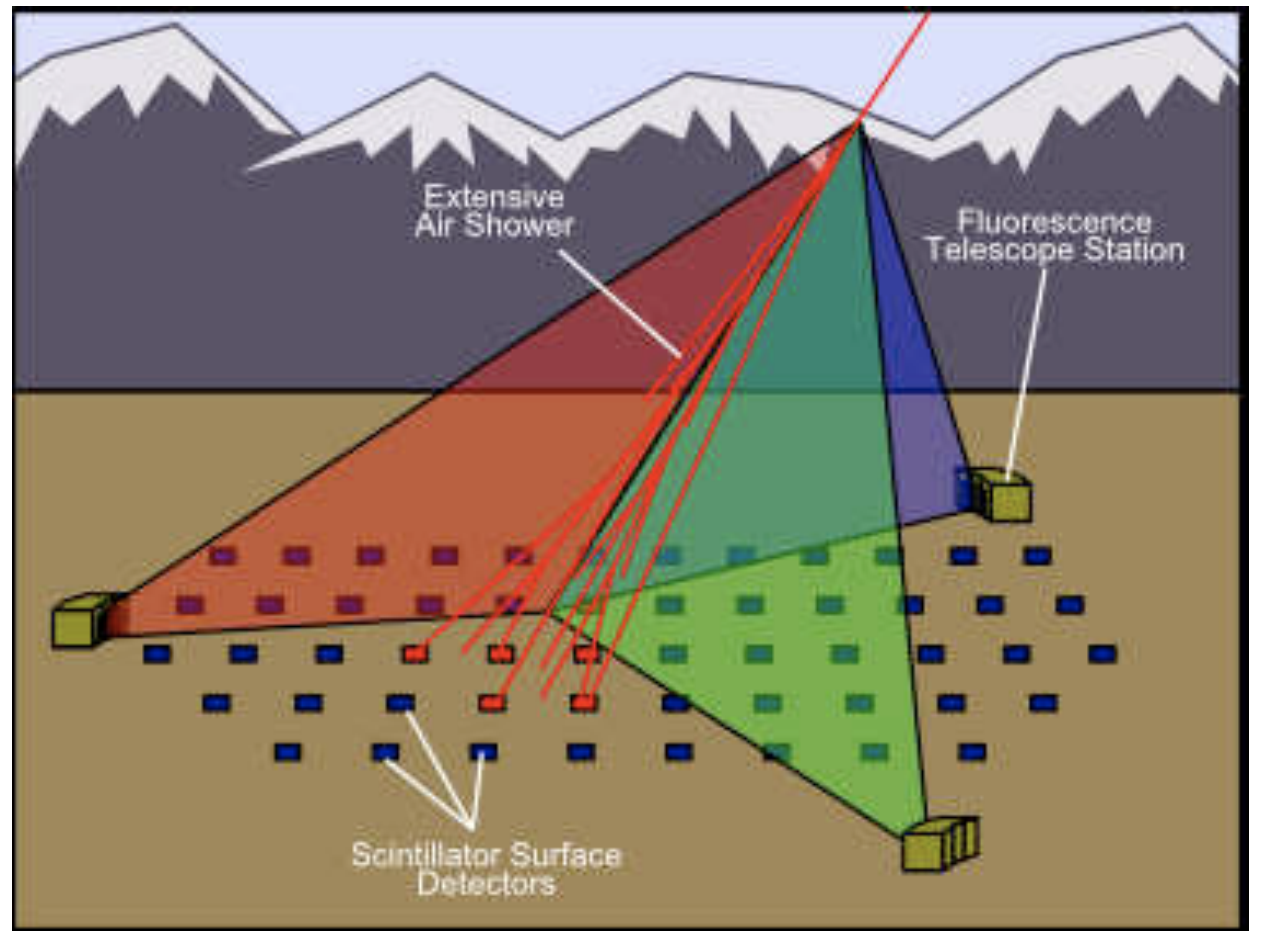

- Location: Utah - $700 \mathrm{~km}^{2}, 500 \mathrm{SD}, 3 \mathrm{FD}, 1.4 \mathrm{~km}$ altitude

- Hybrid designed, based on Akeno SD and HiRes Fly's Eye FD

- In operation: science results compatible $( \pm 1 \sigma)$ with Auger 


\section{UHECR : maximum energy ?}

(Electrical circuit analogy - the real physics boils down to the same)

gyroradius: $\mathrm{r}_{\mathrm{L}} \sim \mathrm{ct}_{\mathrm{gy}} \sim \mathrm{m}_{\mathrm{p}} \mathrm{c}^{2} \gamma / \mathrm{ZeB}=\varepsilon_{\mathrm{p}} / \mathrm{ZeB}<\mathrm{R}$ (size of accel.)

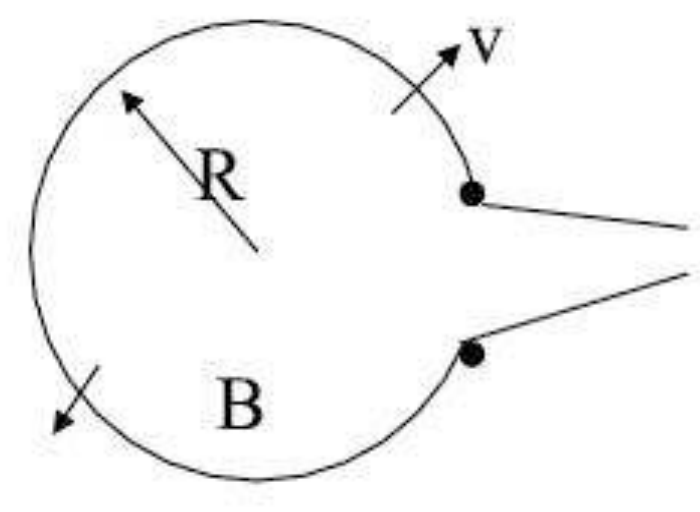

or (EM analog):

$$
V=\frac{1}{c} \dot{\Phi} \sim \frac{1}{c} \frac{B R^{2}}{R / \mathrm{V}}=\beta B R
$$

$$
\longrightarrow \varepsilon_{p}<\beta e B R
$$

$$
\Rightarrow L>4 \pi R^{2} \frac{B^{2}}{8 \pi} v>\frac{1}{2 \beta}\left(\frac{\varepsilon_{p}}{e}\right)^{2} c
$$

But if relativistic expansion, bulk Lorentz factor $\Gamma>>1$, then time ${ }_{\text {obs }} \sim \mathrm{R} / \mathrm{c} \Gamma$, and size $\mathrm{obs}_{\mathrm{o}} \sim \mathrm{R} / \Gamma$, hence need

$$
\Rightarrow L>2 \frac{\Gamma^{2}}{\beta} \varepsilon_{p, 20}^{2} \times 10^{45} \mathrm{erg} / \mathrm{s}
$$

$\Rightarrow$ GRB, AGN..? (only the strongest source types qualify !) 


\section{The Suspects (Hillas plot)}

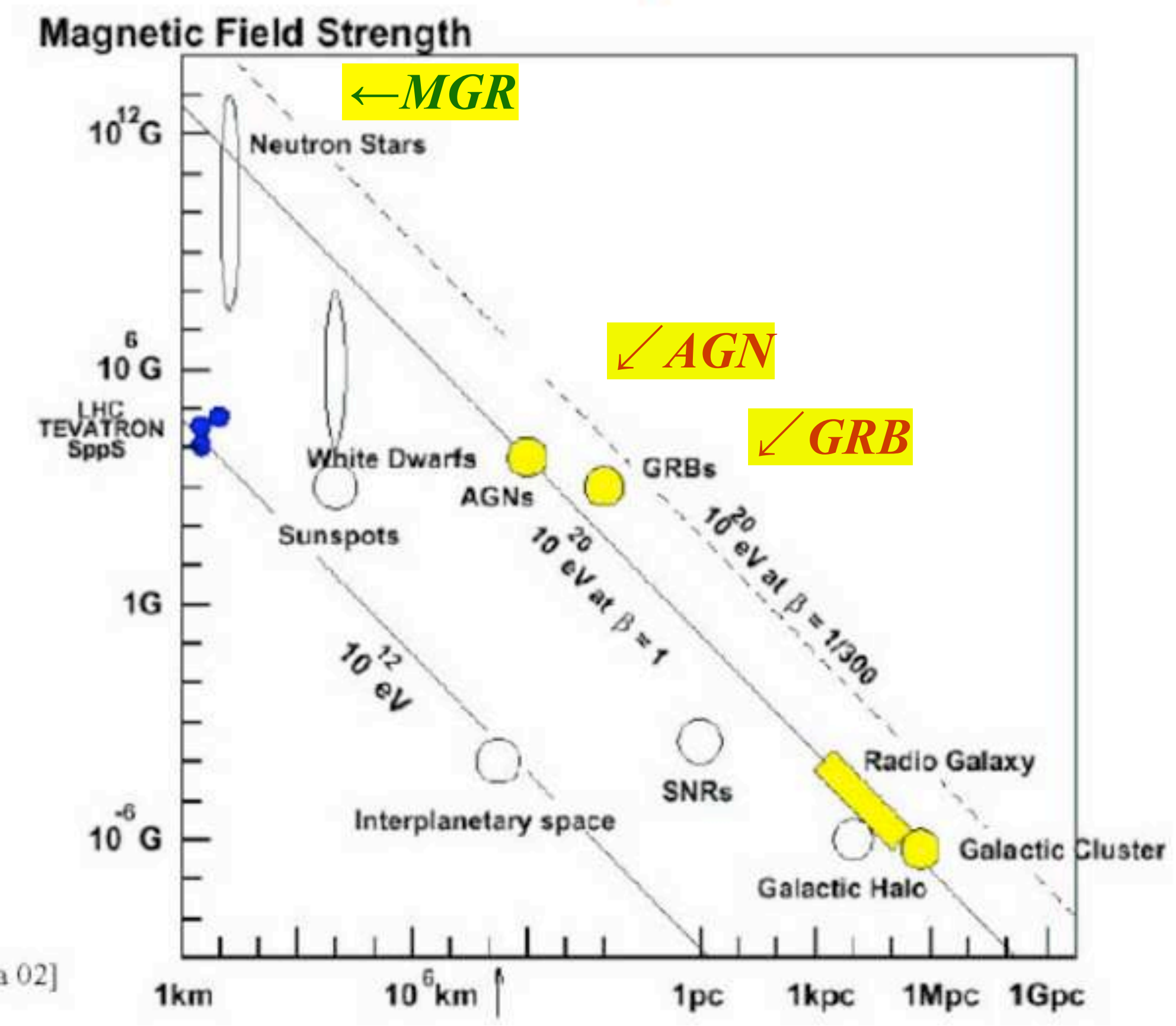




\section{Possible sources of UHECRs (\& Neutrinos) extending to GZK energies}




\section{Astrophysical UHECR Sources?}

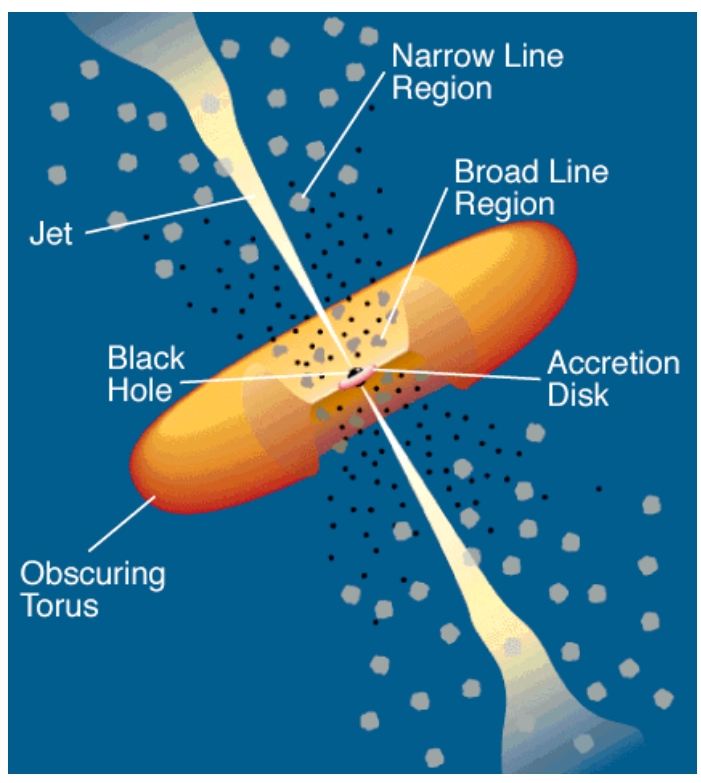

AGN

Active

Galactic

Nucleus

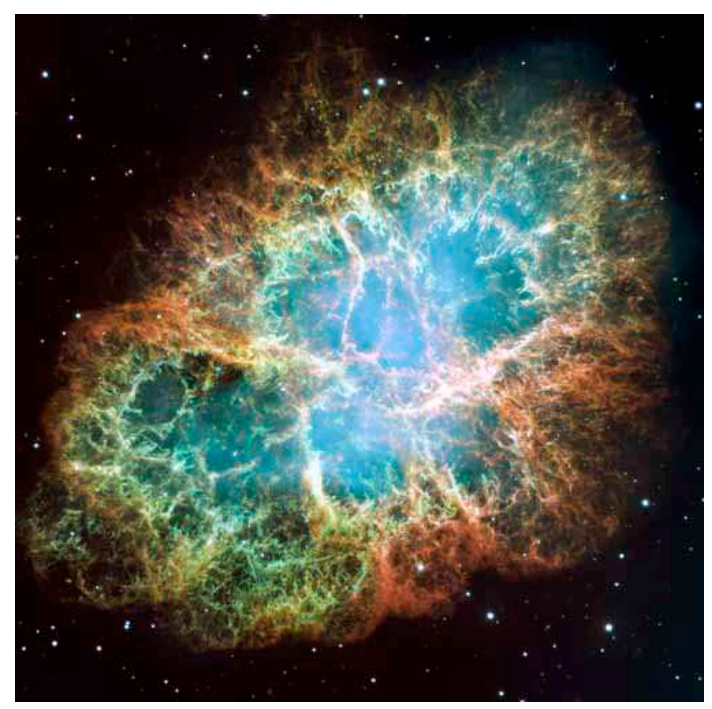

$\mathrm{HN}$

Hypernova

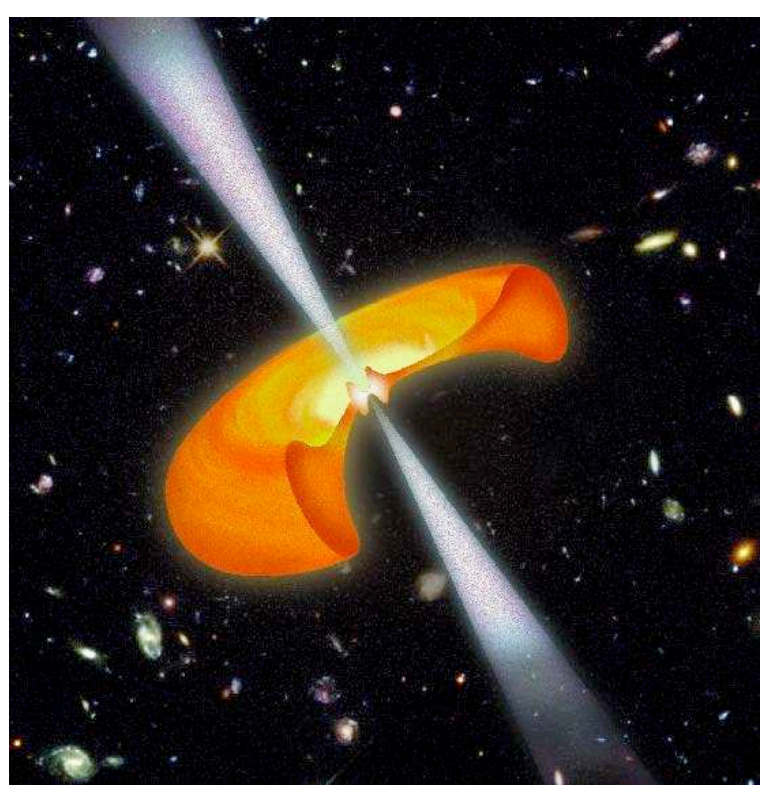

GRB

Gamma

Ray

Burst

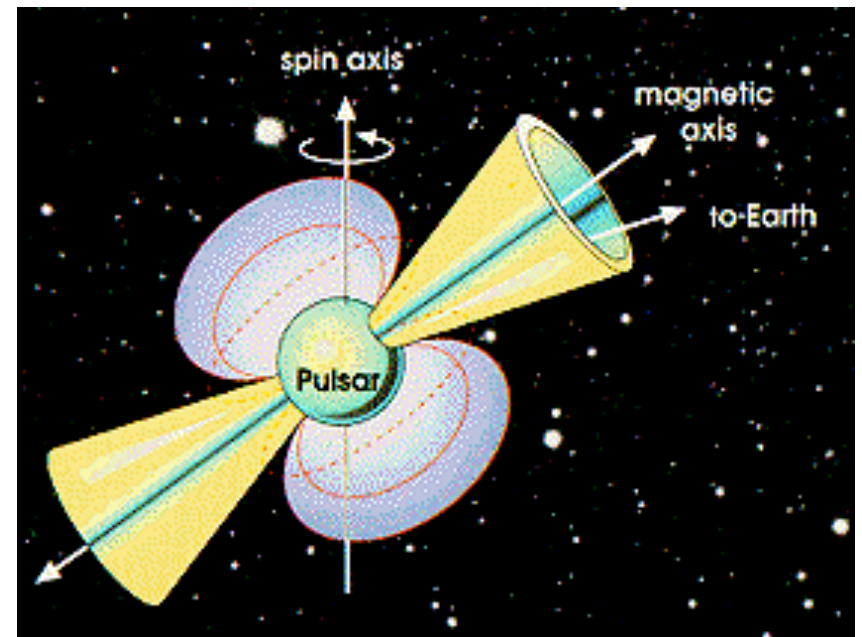

MGR

Magnetar 


\section{Outlook for UHECR}

- The sources of the UHECR are still unknown..!

- They are almost certainly astrophysical sources (not TD)

- $G R B$ remain good candidates, as well as $A G N s, H N e, R Q$, maybe MGRs.

- Will increasingly constrain such possibilities with GeV and $\mathrm{TeV}$ photon observations

- Will learn even more if \& when astrophysical UHENUs are observed from any type of source

- Constraints from diffuse (and intrasource) y-ray emission will also be very useful, and may remain for a long time the main constraint

- Composition and clustering will provide important clues 


\section{What is the Relation} between

\section{UHECRs and UHENUs?}

UHECR = Ultra-High Energy Cosmic Rays UHENU $=$ Ultra-High Energy Neutrinos

$$
\text { define } \quad \begin{aligned}
\mathrm{HE} & \approx 10^{9} \mathrm{eV}(\mathrm{GeV}) \\
\mathrm{VHE} & \approx 10^{12} \mathrm{eV}(\mathrm{TeV}) \\
\mathrm{UHE} & \approx 10^{18} \mathrm{eV}(\mathrm{EeV})
\end{aligned}
$$




\section{TeV Neutrinos}

Observing astrophysical neutrinos allows conclusions about the acceleration mechanism of Cosmic Rays

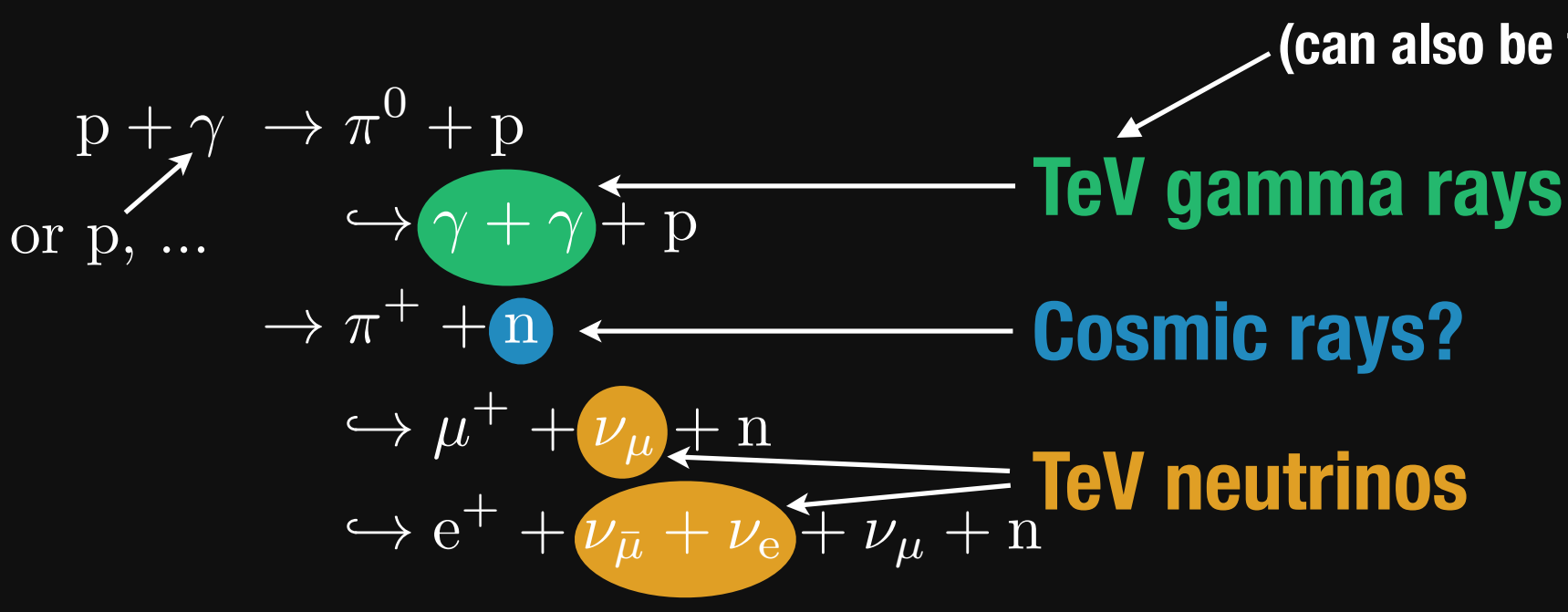

Deutrinos from cosmic ray interactions in:

- Atmosphere

- Cosmic Microwave Background

- Gamma Ray Bursts (Acceleration Sites)

- Active Galactic Nuclei (Acceleration Sites)

- ?

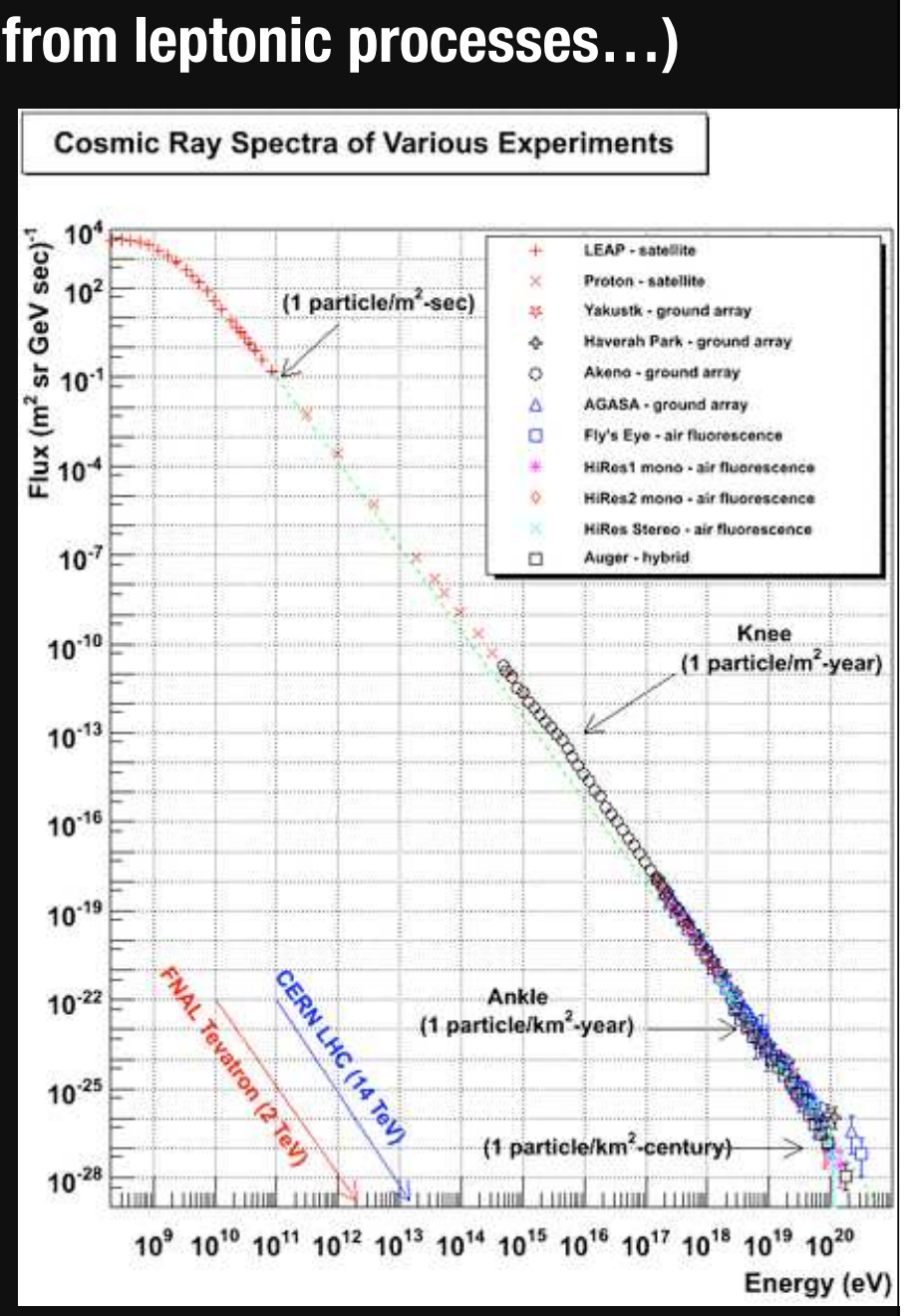

Slides: C. Kopper 14 Moriond 


\section{Astro VHE neutrinos}

At the simplest level:

- Fermi acceleration: particle power law $\mathrm{dN}_{\mathrm{p}, \mathrm{e}} / \mathrm{dE} \sim \mathrm{E}^{-\mathrm{q}}$

- $\mathrm{e}^{ \pm}, \mathrm{B} \rightarrow \gamma \quad(\mathrm{PL} \gamma \mathrm{s}$, act as targets for the accelerated $\mathrm{P})$

- $\mathrm{p}, \mathrm{\gamma} \rightarrow \pi^{ \pm} \rightarrow \mu^{ \pm}, \mathrm{V}_{\mu} \rightarrow \mathrm{e}^{ \pm}, \mathrm{V}_{\mathrm{e}}, \mathrm{V}_{\mu}$

- For PL $d N_{e, p} / d E$ and $d N_{\gamma} / d E \rightarrow d N_{v} / d E$ also $P L$

- Parameters: $\epsilon_{\mathrm{p}}, \epsilon_{\mathrm{e}}, \epsilon_{\mathrm{B}}$ : energy ratios of p,e,B to $E_{\text {tot }}$

- $\mathrm{E}_{\text {tot: }}$ total burst energy, Г: bulk Lorentz factor 


\section{How can one detect UHENUs?}




\section{$\nu$ Interactions}

- Due to SM Weak Interactions

$$
\sigma^{\nu p} \sim 10^{-38} \mathrm{~cm}^{2} \frac{E_{\nu}}{\mathrm{GeV}}
$$

- Let's consider for example atmospheric $\nu^{\prime} s$ ?

$$
\Phi_{\nu}^{\mathrm{ATM}}=1 \nu \text { per } \mathrm{cm}^{2} \text { per second and }\left\langle E_{\nu}\right\rangle=1 \mathrm{GeV}
$$

- How many interact? In a human body:

$$
\begin{aligned}
& N_{\text {int }}=\Phi_{\nu} \times \sigma^{\nu p} \times N_{\text {prot }}^{\text {human }} \times T_{\text {life }}^{\text {human }}(M \times T \equiv \text { Exposure }) \\
& \left.\begin{array}{l}
N_{\text {protons }}^{\text {human }}=\frac{M^{\text {human }}}{g r} \times N_{A}=80 \mathrm{~kg} \times N_{A} \sim 5 \times 10^{28} \text { protons } \\
T^{\text {human }}=80 \text { years }=2 \times 10^{9} \mathrm{sec}
\end{array}\right\} \begin{array}{r}
\text { Exposure }_{\text {human }} \\
\sim \text { Ton } \times \text { year }
\end{array} \\
& N_{\text {int }}=\left(5 \times 10^{28}\right)\left(2 \times 10^{9}\right) \times 10^{-38} \sim 1 \text { interaction per lifetime }
\end{aligned}
$$$$
\Rightarrow \text { Need huge detectors with Exposure } \sim \text { KTon } \times \text { year }
$$ 
Amundsen-Scott South Pole station

\section{Antarctica}

\section{IceCube}

for >TeV v's:

GTon detector

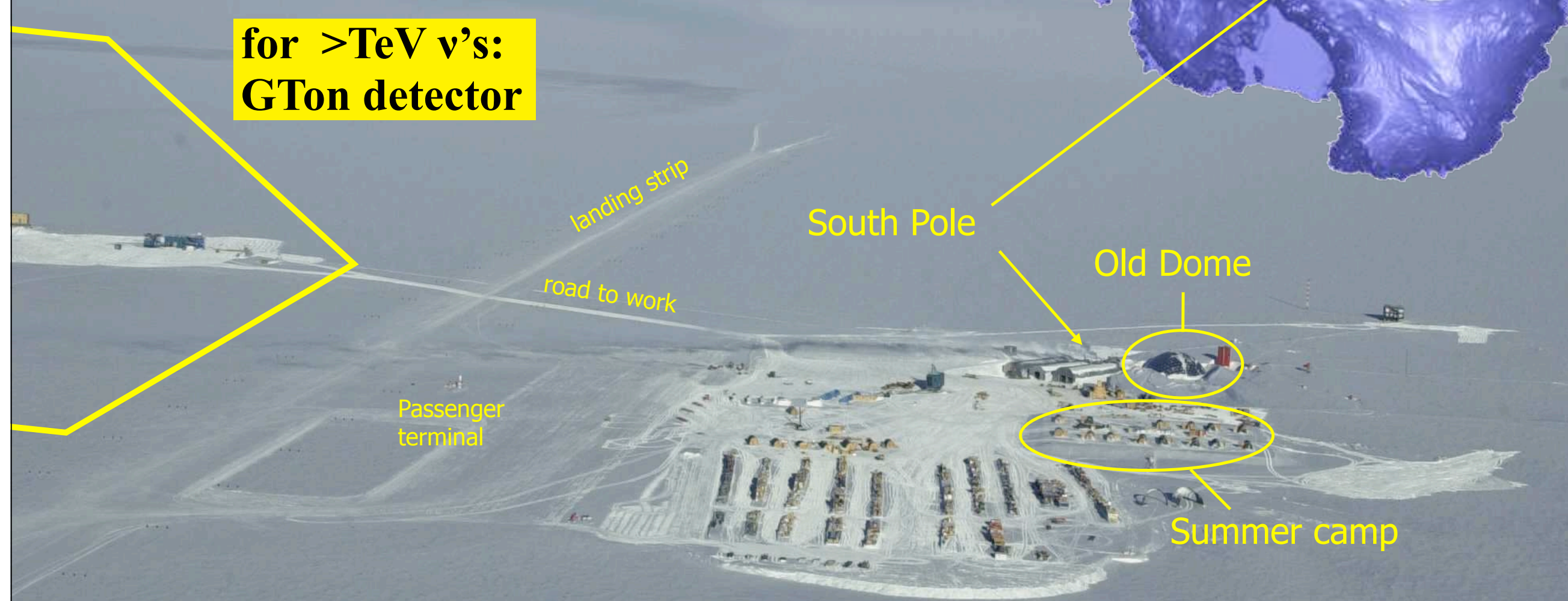

(IceCube slides credit:

IceCube collaboration) 


\section{The IceCube Collaboration}

http://icecube.wisc.edu

\section{6 institutions, 270 members}

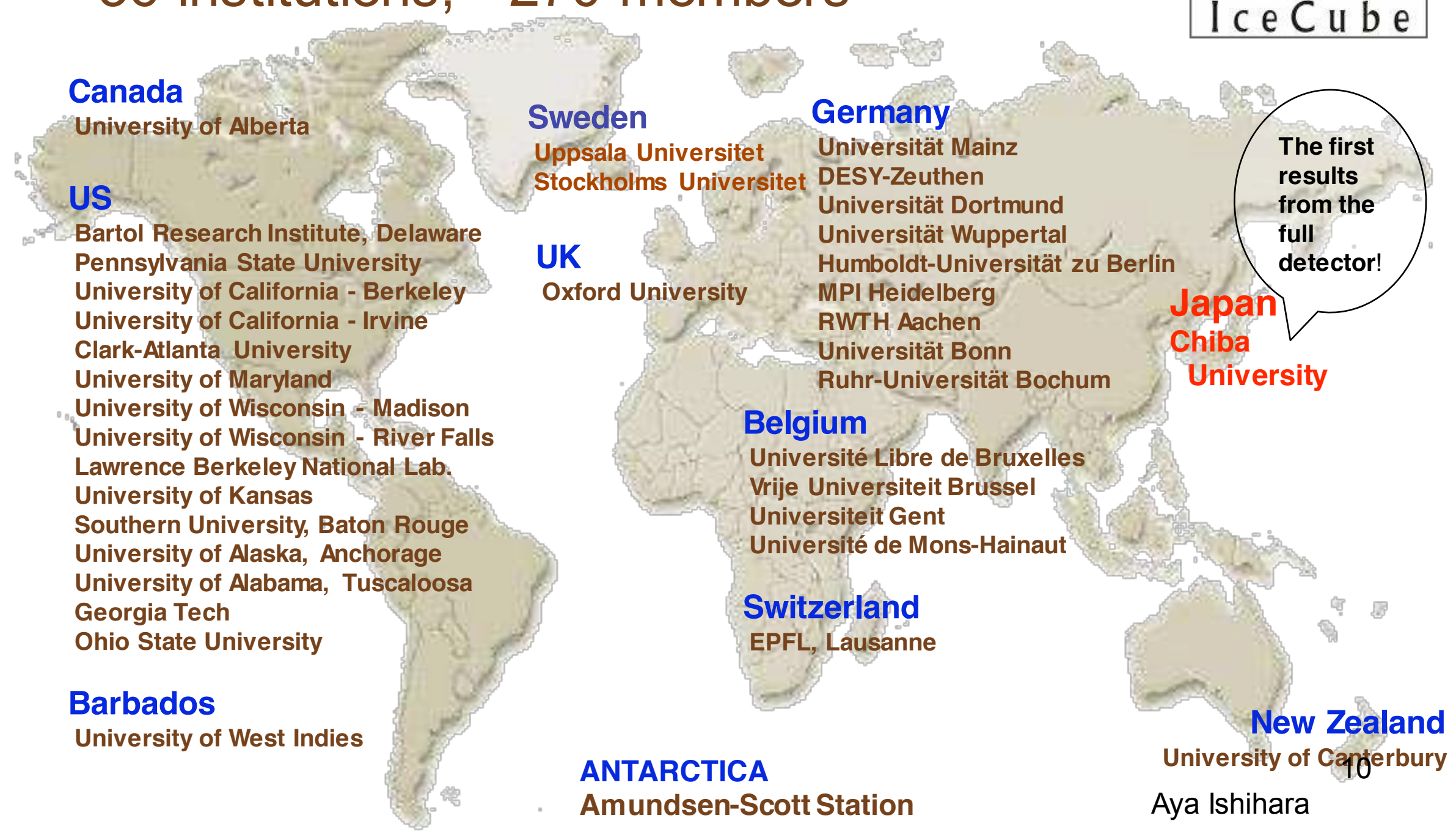




\section{Why The South Pole?}

- Deep $(3 \mathrm{~km})$ clear ice

- on land (not on water as at north pole)

- Excellent infrastructure new south pole station

No distractions, easy to focus on work No polar bears 
36 institutions, $\sim 270$ members, 10 countries:

\section{ICECUBE}

5160 DOMs on 86 strings

160 tank ice-Cherenkov surface air shower array (IceTop) -

Includes DeepCore infill array (sensitivity to lower energies)

i 86 strings - completed in 2010

\section{Digital Optical Module (DOM)}

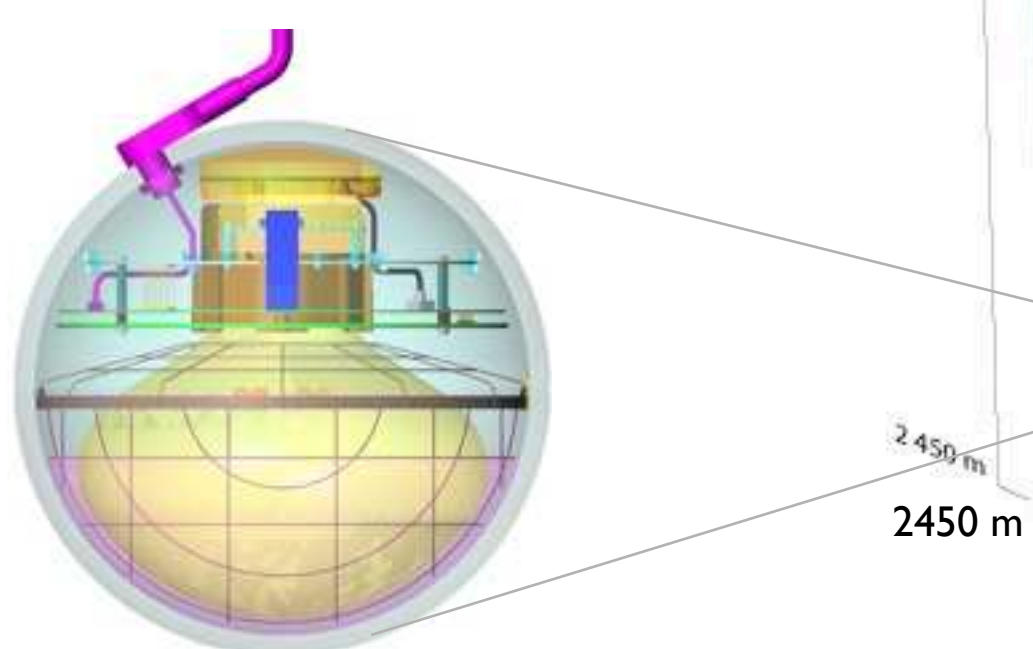

I GTon instrumented volume, USD 300M (30c/Ton= $80 \mathrm{ft} /$ tonna) 


\section{Neutrino Telescopes}

I ce Cube

- Neutrinos interact in or near the detector

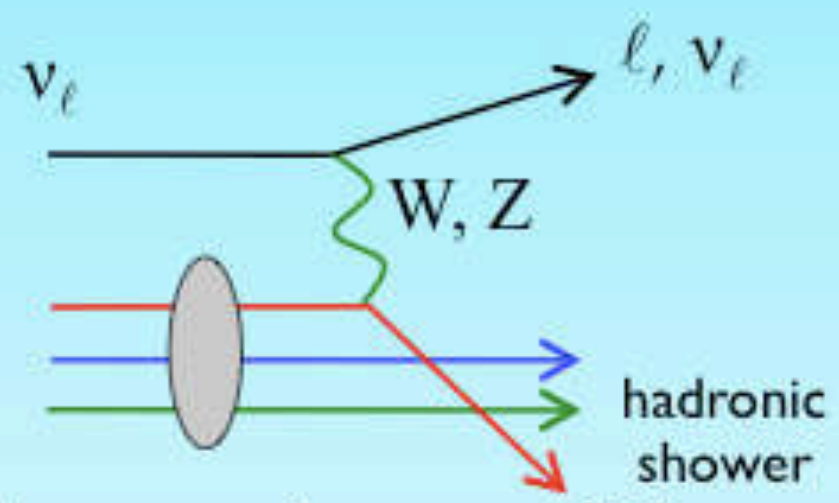

- $\mathcal{O}(\mathrm{km})$ muons from $v_{\mu}(\mathrm{CC})$

- $\mathrm{O}(10 \mathrm{~m})$ particle cascades from $\mathrm{v}_{\mathrm{e}}$, low energy $\mathrm{v}_{\mathrm{T}}$, and $\mathrm{NC}$ interactions

- Cherenkov radiation detected by optical sensors

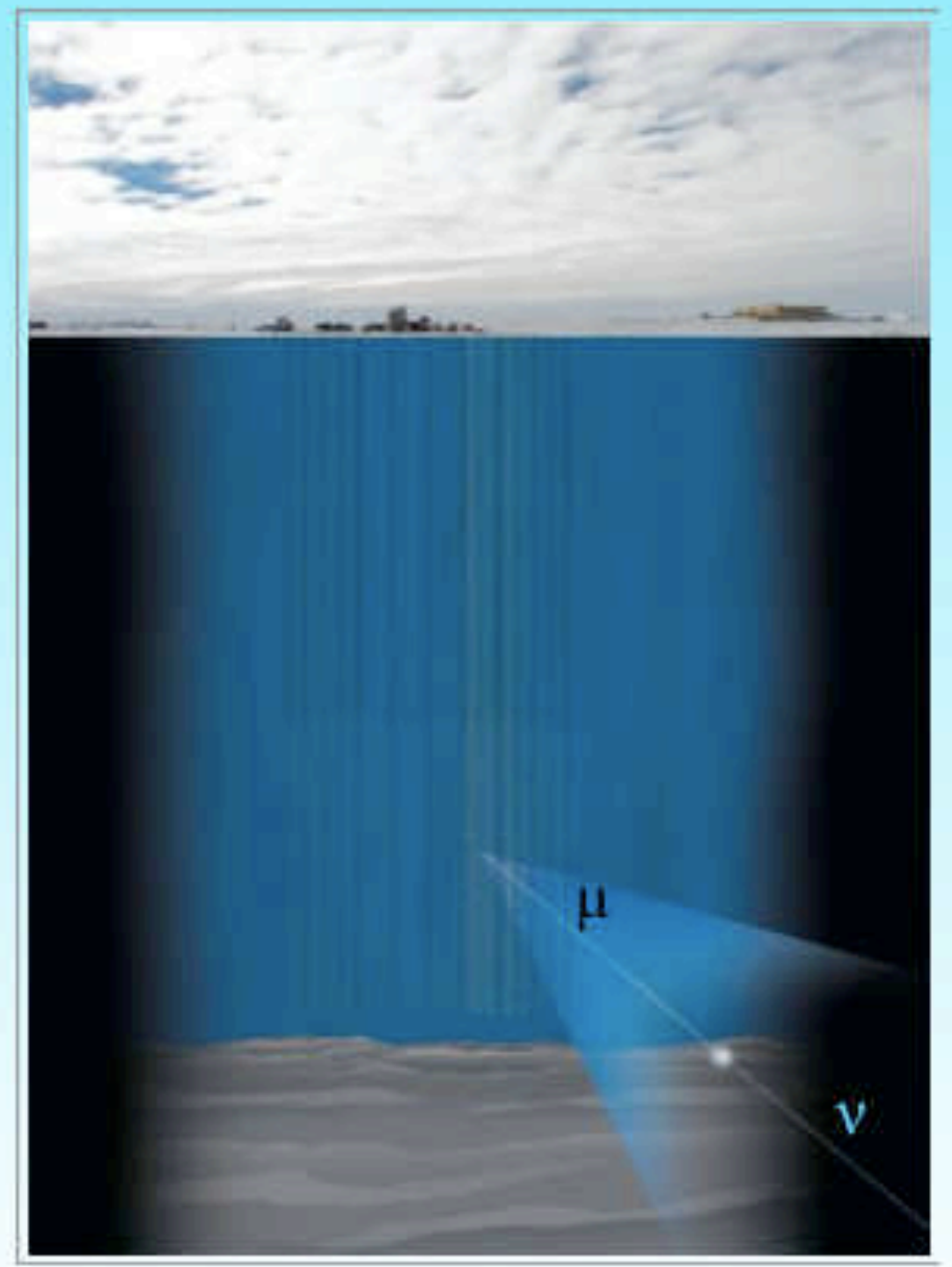




\section{Signals and Backgrounds}

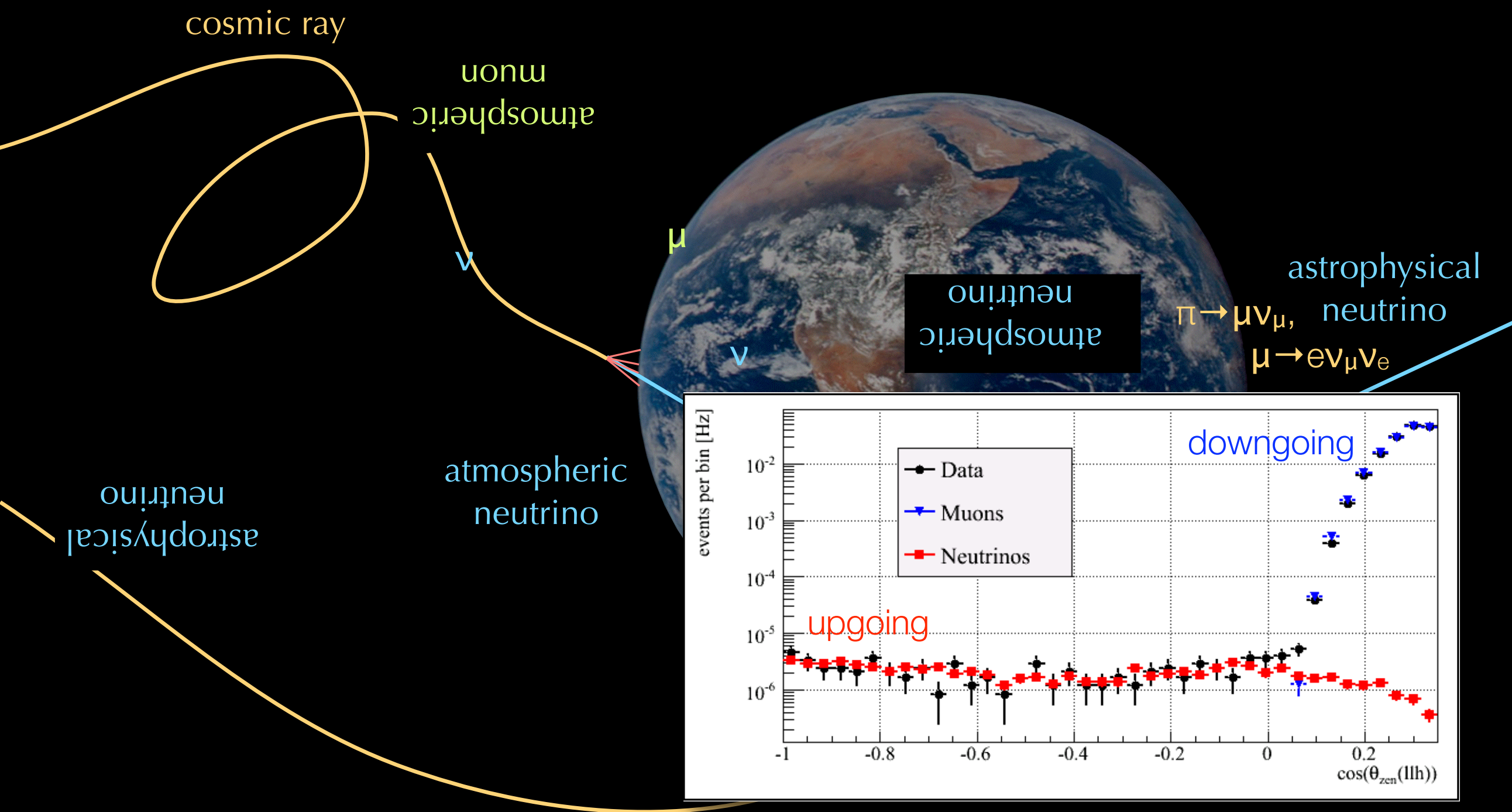




\section{IceCube sees the same Substance from its control room as its Competitor, ANTARES}

BUT: View from ANTARES Control Room

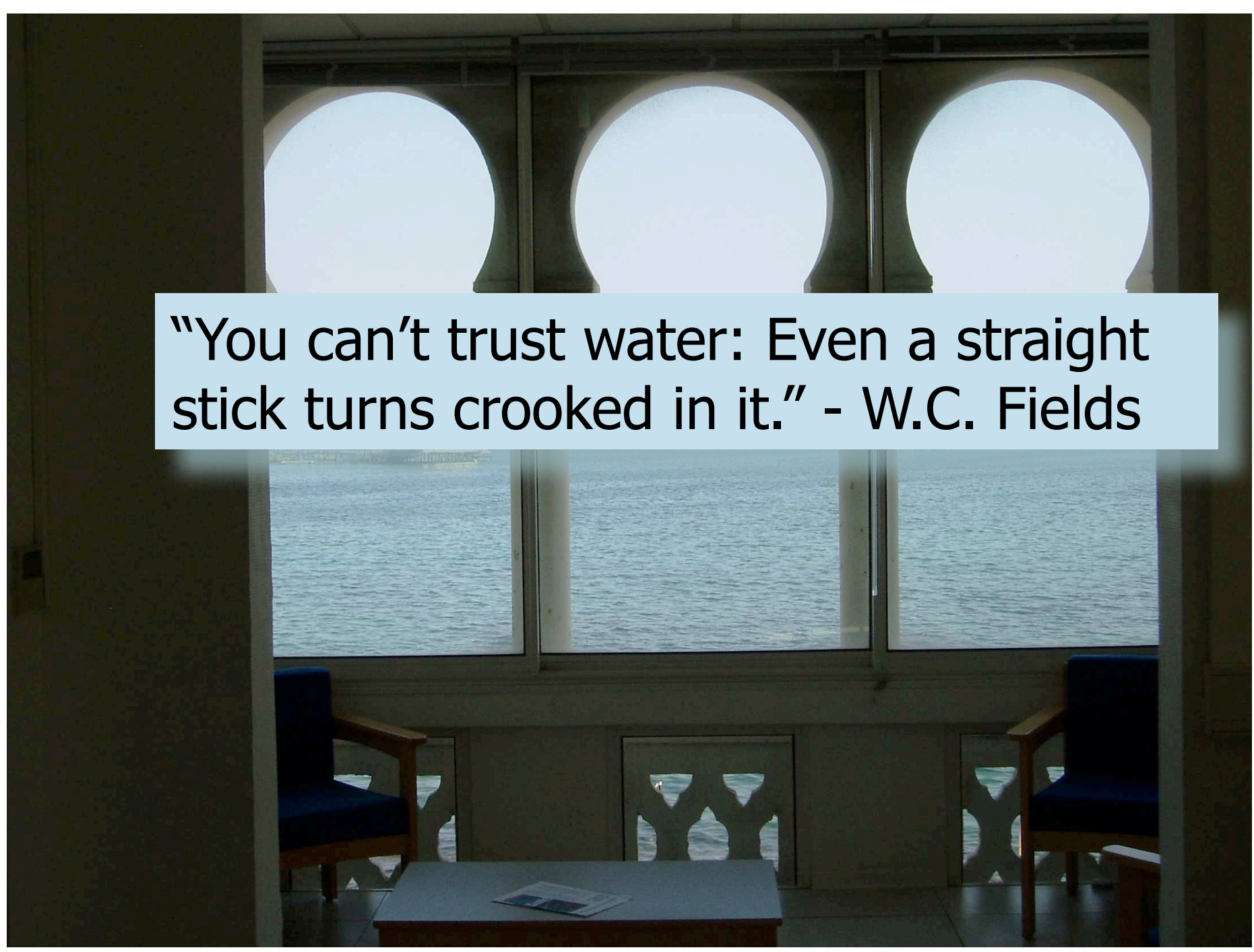

ANTARES (off the Mediterranean coast of southern France- almost Club Med..) successfully built a working 12-line neutrino telescope . Small $\left(0.15 \mathrm{~km}^{3}\right)$ compared to IceCube, but... 


\section{Antares is a prototype for the Next Big EU Nu-detector:}

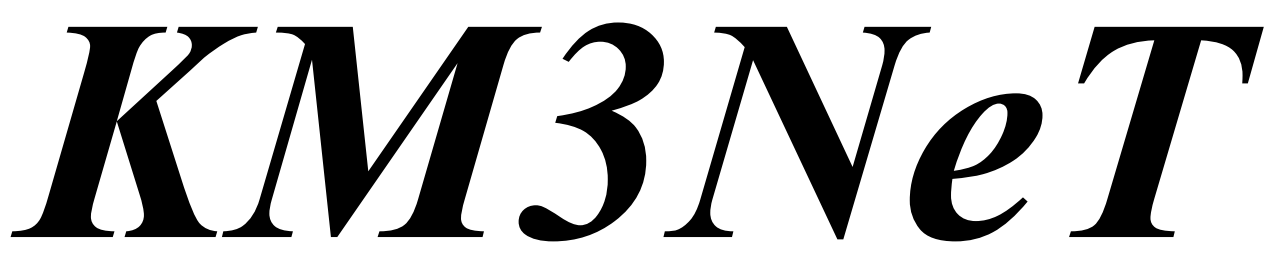




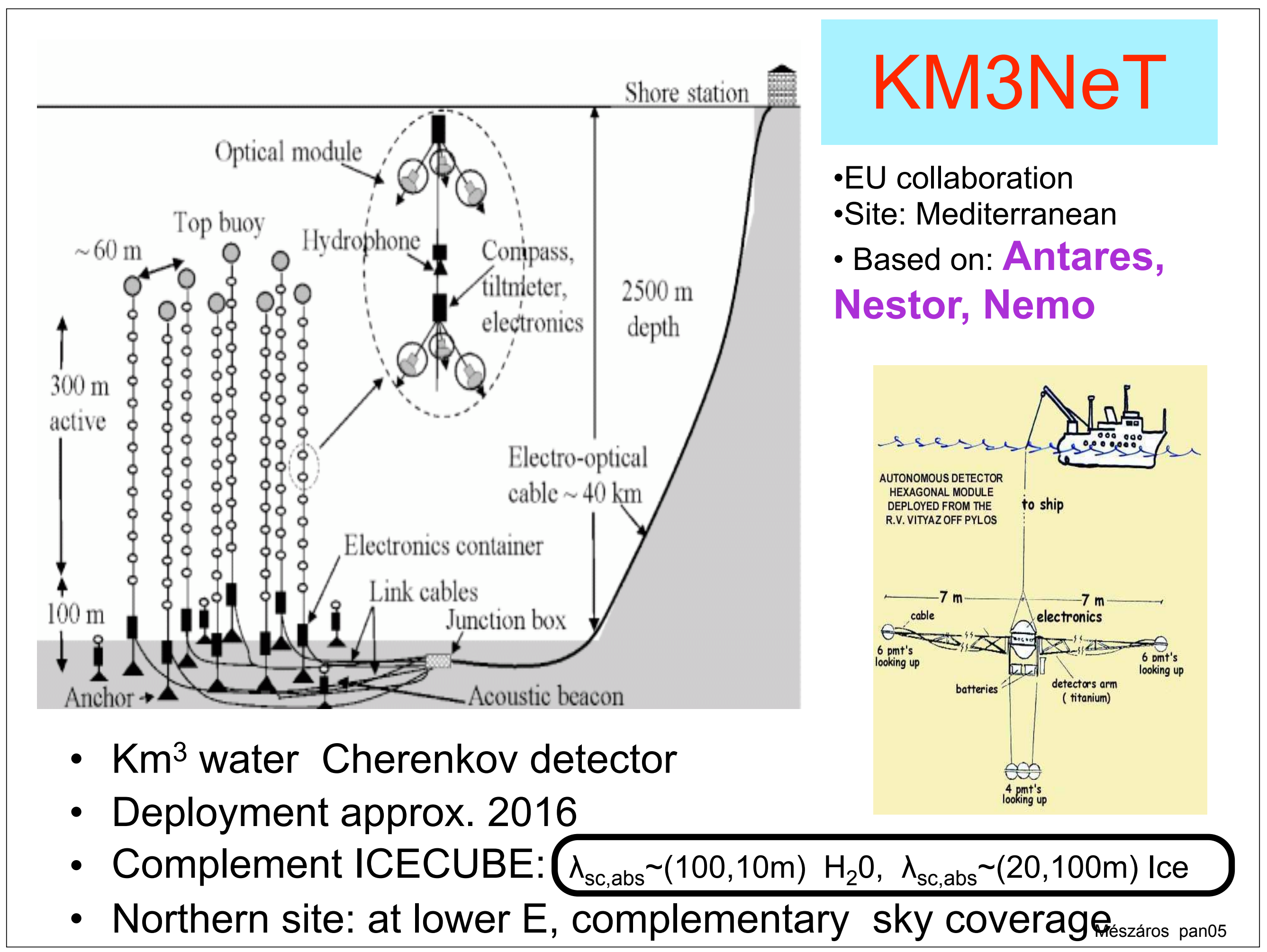




\section{KM3NeT : 2016}

Total volume $\sim 4$ km³ 3 Mediterranean sites: France, Italy, Greece

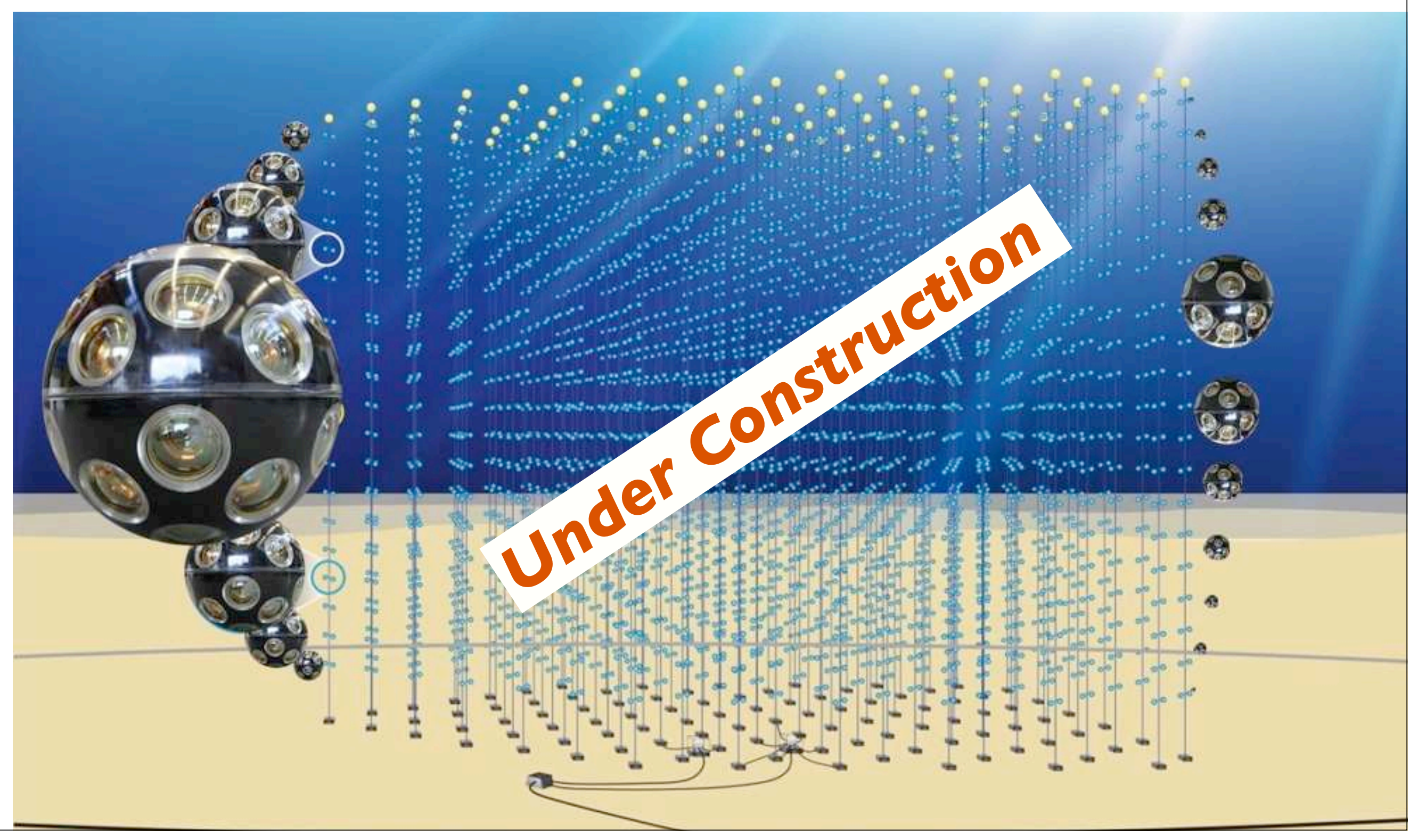




\section{Neutrino Event Signatures}

Signatures of signal events

\section{GG Muon Neutrino}

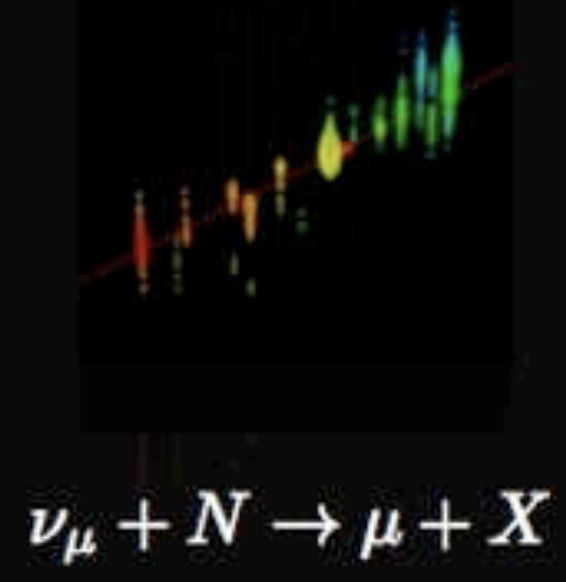

track (data)

factor of $\approx 2$ energy resolution $<1^{\circ}$ angular resolution at high energies

\section{Neutral Gurrent / Electron Neutrino}

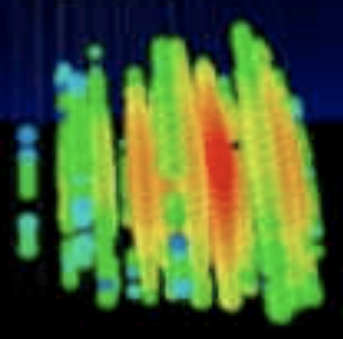

$$
\begin{aligned}
& \nu_{\mathrm{e}}+N \rightarrow \mathrm{e}+X \\
& \nu_{\mathrm{x}}+N \rightarrow \nu_{\mathrm{x}}+X
\end{aligned}
$$

cascade (data)

$\approx \pm 15 \%$ deposited energy resolution

$\approx 10^{\circ}$ angular resolution (at energies ₹ $100 \mathrm{TeV}$ )

\section{GG Tau Neutrino}

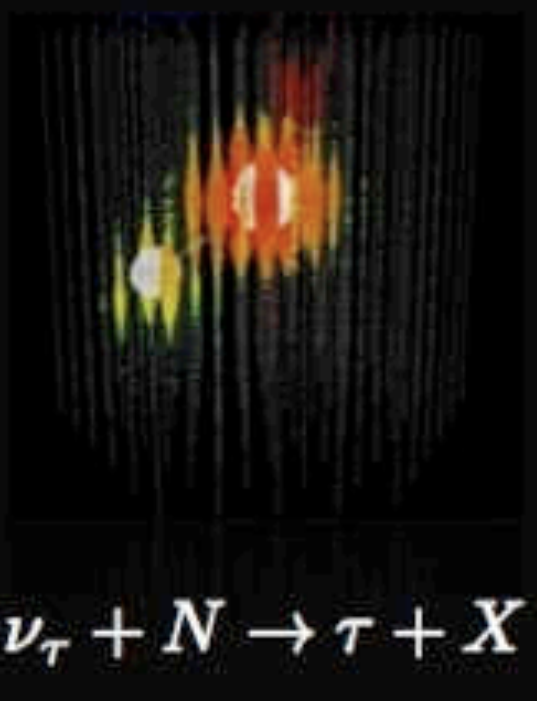

"double-bang" ( $(10 \mathrm{PeV})$ and other signatures (simulation)

(not observed yet) 


\section{Flavor composition at source}

- Pionic:

$p, \gamma(p, p) \rightarrow \pi^{+} \rightarrow \mu^{+}, \nu_{\mu} \rightarrow e^{+}, \bar{\nu}_{\mu}, \nu_{e} \rightarrow[1 ; 2 ; 0]_{s r c}$

- Damped muons :

$\pi^{+} \rightarrow \mu^{+}, \nu_{\mu} \quad(+$ cooled muons $) \rightarrow[0 ; 1 ; 0]_{\text {src }}$

- Prompt :

$\pi^{+}$(dense : interact before decay $) \rightarrow[1 ; 1 ; 0]_{s r c}$

- Beta beam :

(neutron decay) $n \rightarrow p^{+}, e^{-}, \bar{\nu}_{e} \rightarrow[1 ; 0 ; 0]_{\text {src }_{68}}$ 


\section{Flavor oscillations in vacuum}

Vacuum oscillations: $[\mathrm{i}, \mathrm{j}, \mathrm{k}]_{\mathrm{obs}}=\mathrm{P}_{\mathrm{osc}} \cdot[\mathrm{i}, \mathrm{j}, \mathrm{k}]_{\mathrm{src}}$, where "tri-bi-maximal" vac. osc. probability matrix

$$
P_{T B M} \simeq \frac{1}{18}\left[\begin{array}{lll}
10 & 4 & 4 \\
4 & 7 & 7 \\
4 & 7 & 7
\end{array}\right]
$$

Thus, approximate flavor composition observed is:

- Pionic:

$\mathrm{P}_{\mathrm{TBM}}[1,2,0]_{\mathrm{src}}=[1 ; 1 ; 1]_{\mathrm{obs}}$ Damped muons: $\mathrm{P}_{\mathrm{TBM}}[0,1,0]_{\mathrm{src}}=[1 ; 1.8 ; 1.8]_{\mathrm{obs}}$, Prompt (dense): $\quad$ Р $_{\text {твм }}[1,1,0]_{\mathrm{src}}=[1 ; 0.6 ; 0.6]_{\mathrm{obs}}$, Beta beam: $\quad$ Р $_{\text {твм }}[1,0,0]_{\mathrm{src}}=[5 ; 2 ; 2]_{\mathrm{obs}}$ 


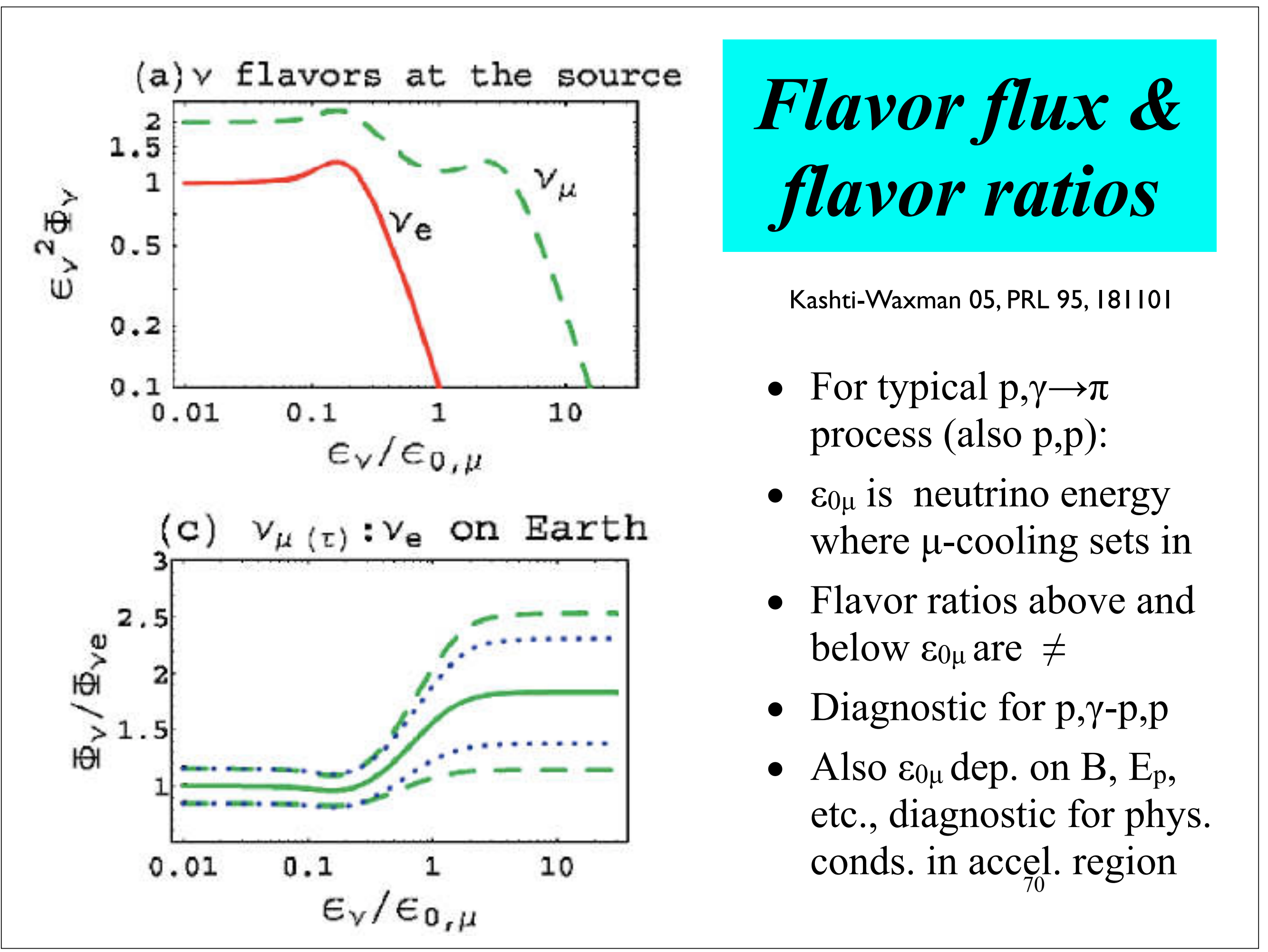




\section{Deep Core : fundamental physics}

\section{Using atmospheric neutrinos}

- muon neutrino disappearance: feasable

- tau neutrino appearance : reasonable

- neutrino mass hierarchy : difficult

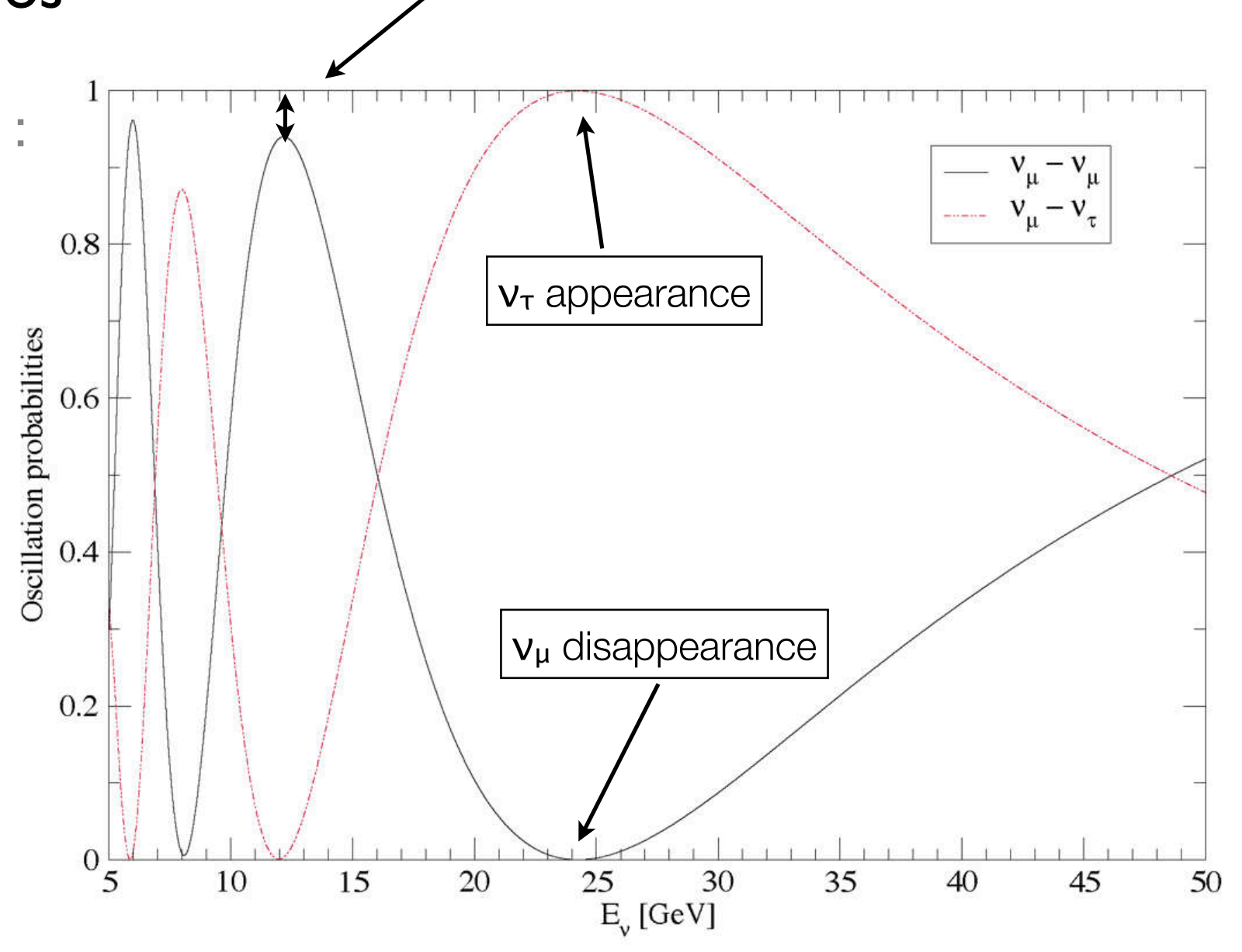

Mena, Mocioiu \& Razzaque 08, PRD 78, 093003

for vertically up-going neutrinos 


\section{and finally .... Science! \\ late 2013: ICECUBE announced}

The first detection of "certified" astrophysical

\section{neutrinos}




\title{
Non-atmospheric PeV nus: extragalactic CR tracers?
}

\author{
IceCube's first PeV events
}
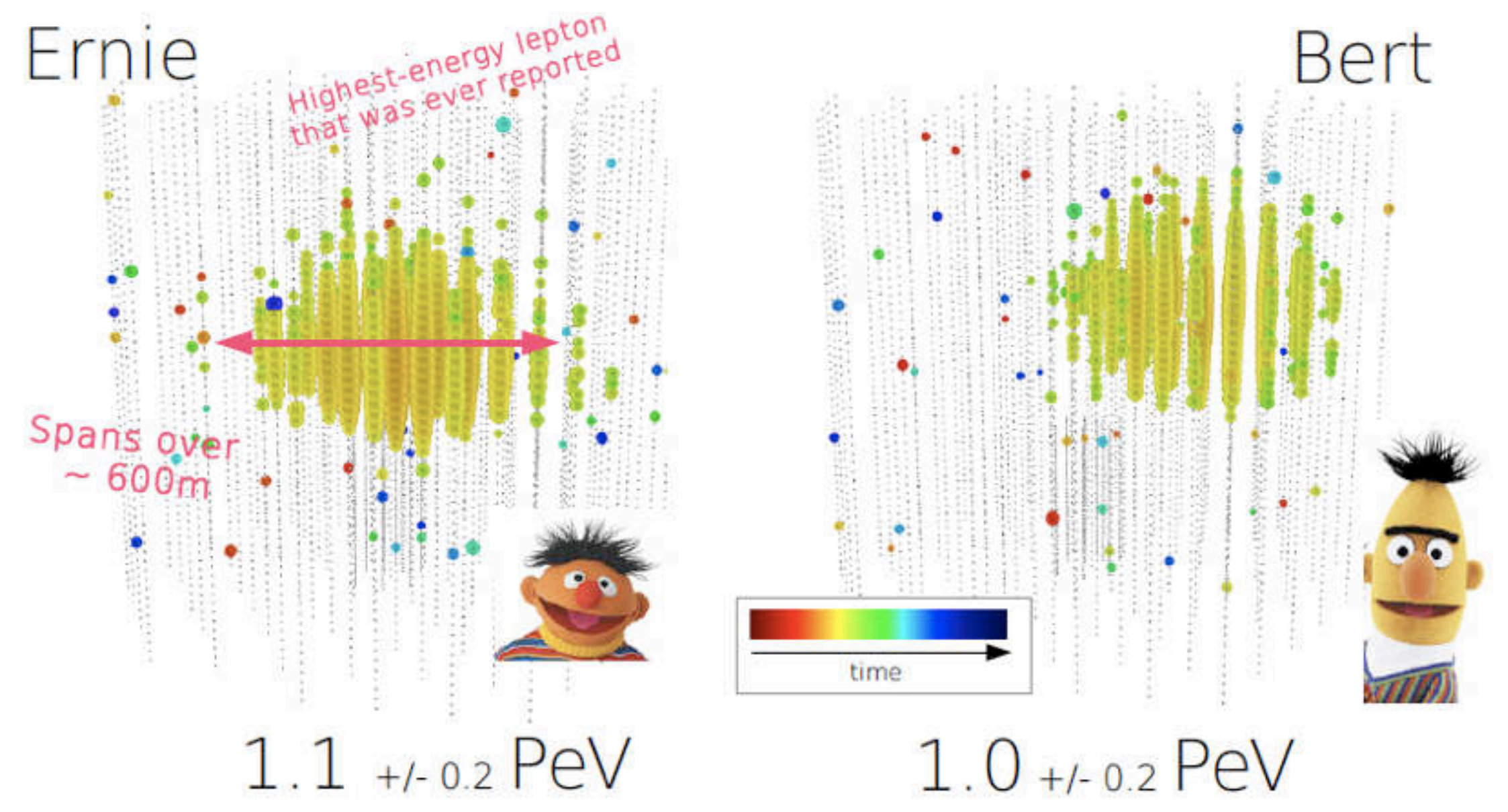


\section{Updated HESE Results (3 Year)}

- 988 day sample

(Sullivan, Beyond IceCube 2014)

- detected 37 events

- expected background of $8.4 \pm 4.2$ cosmic ray muon events and $6.6+5.9$ atmospheric neutrinos.

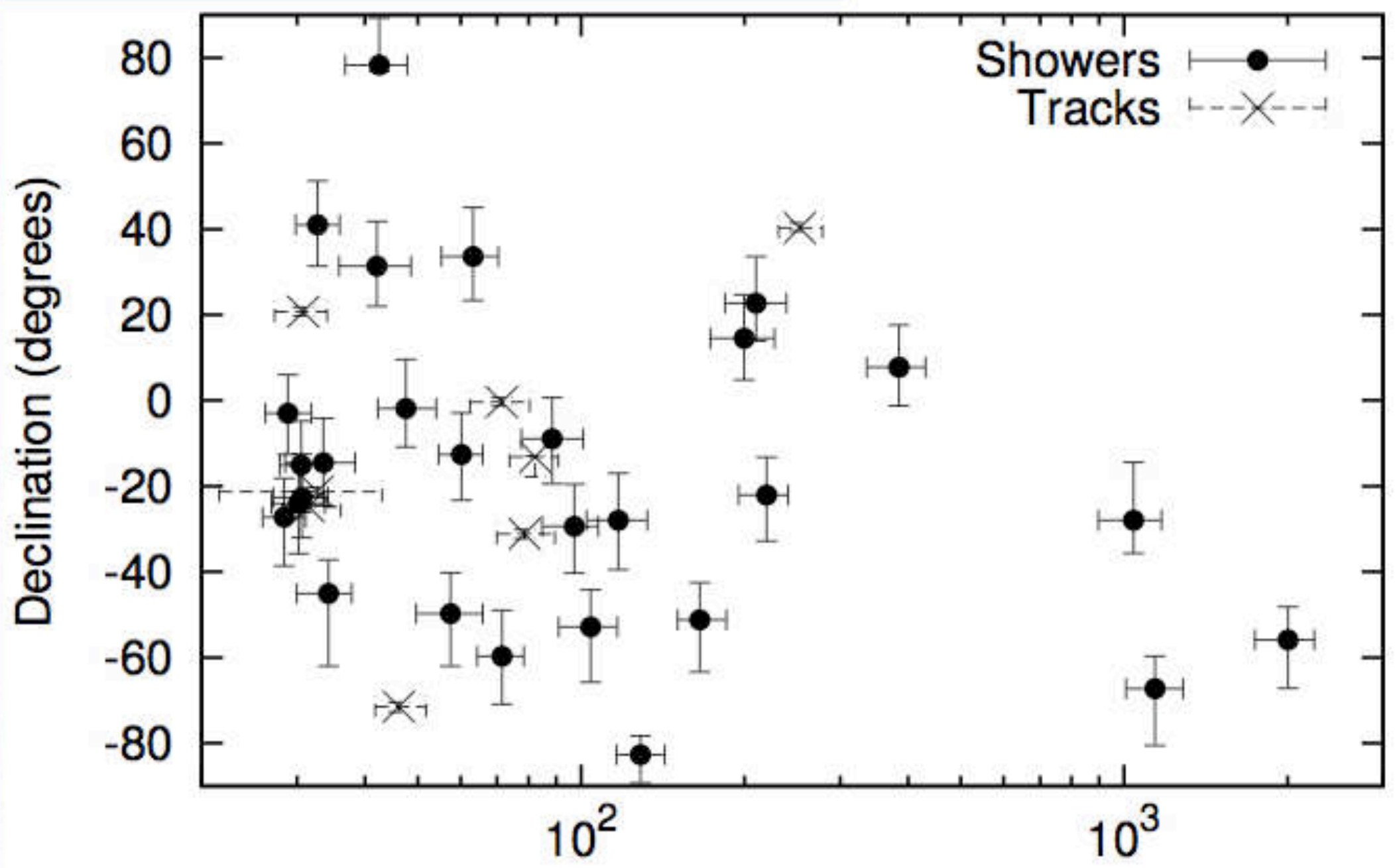

Deposited EM-Equivalent Energy in Detector $(\mathrm{TeV})$ 


\section{Atmospheric neutrino flux and diffuse limit}

- high-energy atmospheric $\nu_{\mu} / \nu_{e}$-spectrum as seen by IC-40 \& IC-79/DC

[IceCube'11,'12]

- predicted prompt atmospheric $\nu$-fluxes (charmed meson decay)

[Enberg et al.'08]

$\rightarrow$ high-energy starting event (HESE) analysis

[IceCube Science'13]

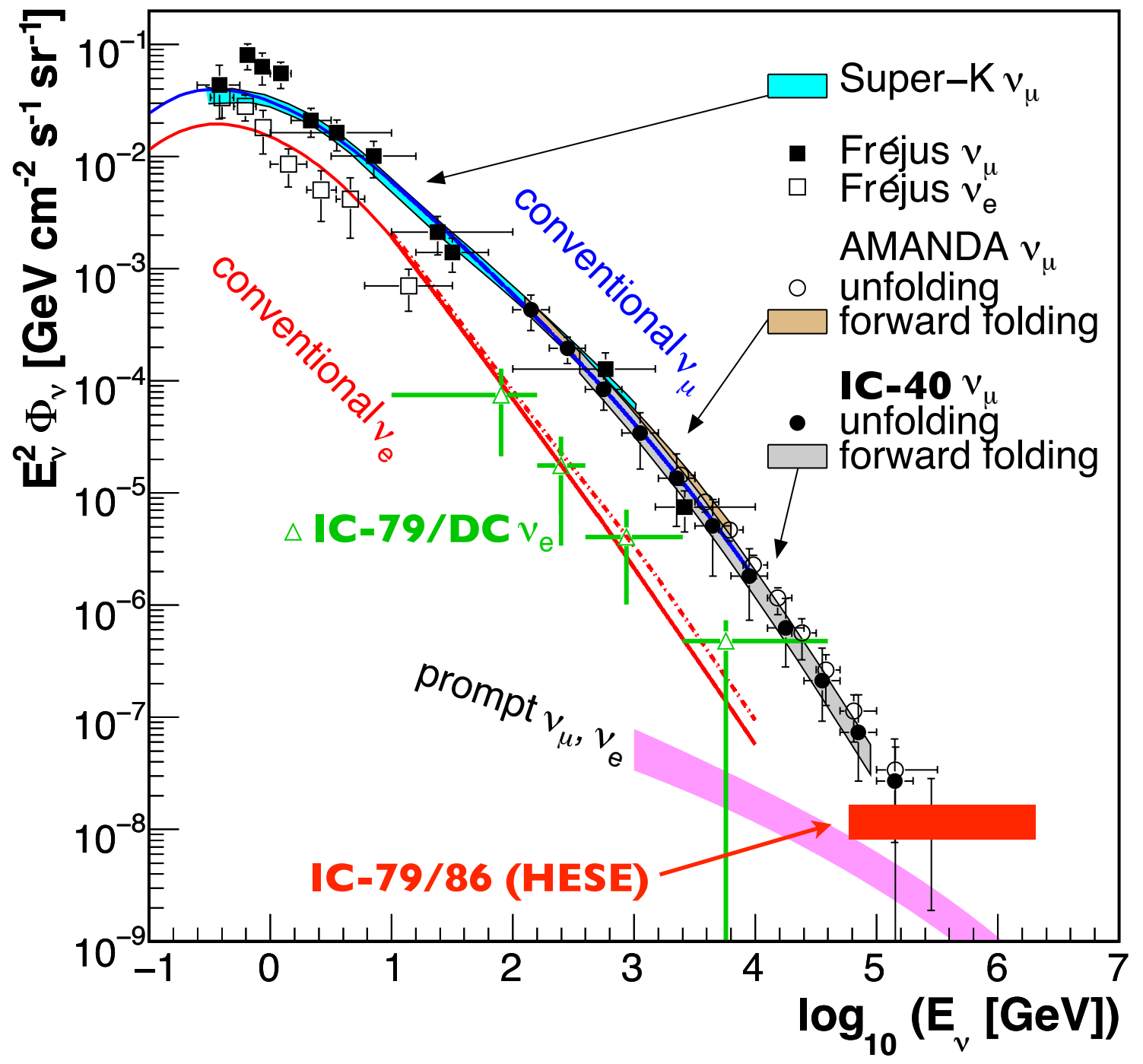




\section{IC79,86- $1 v_{\mu}$ diffuse neutrinos}

(Sullivan, Beyond IceCube 20I4)

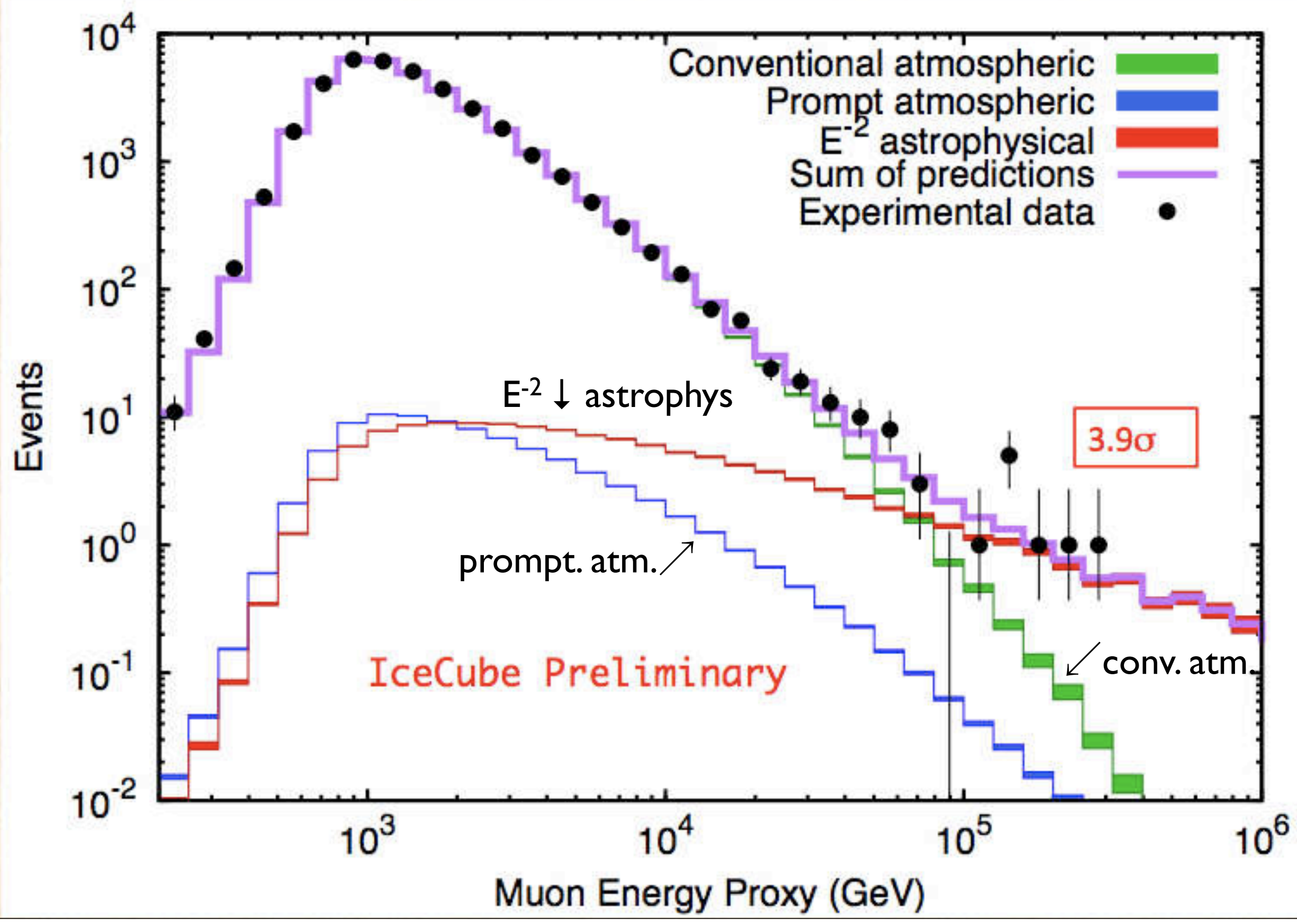




\section{HESE 3 Year Results \\ Any obvious sources?}

Not really...!

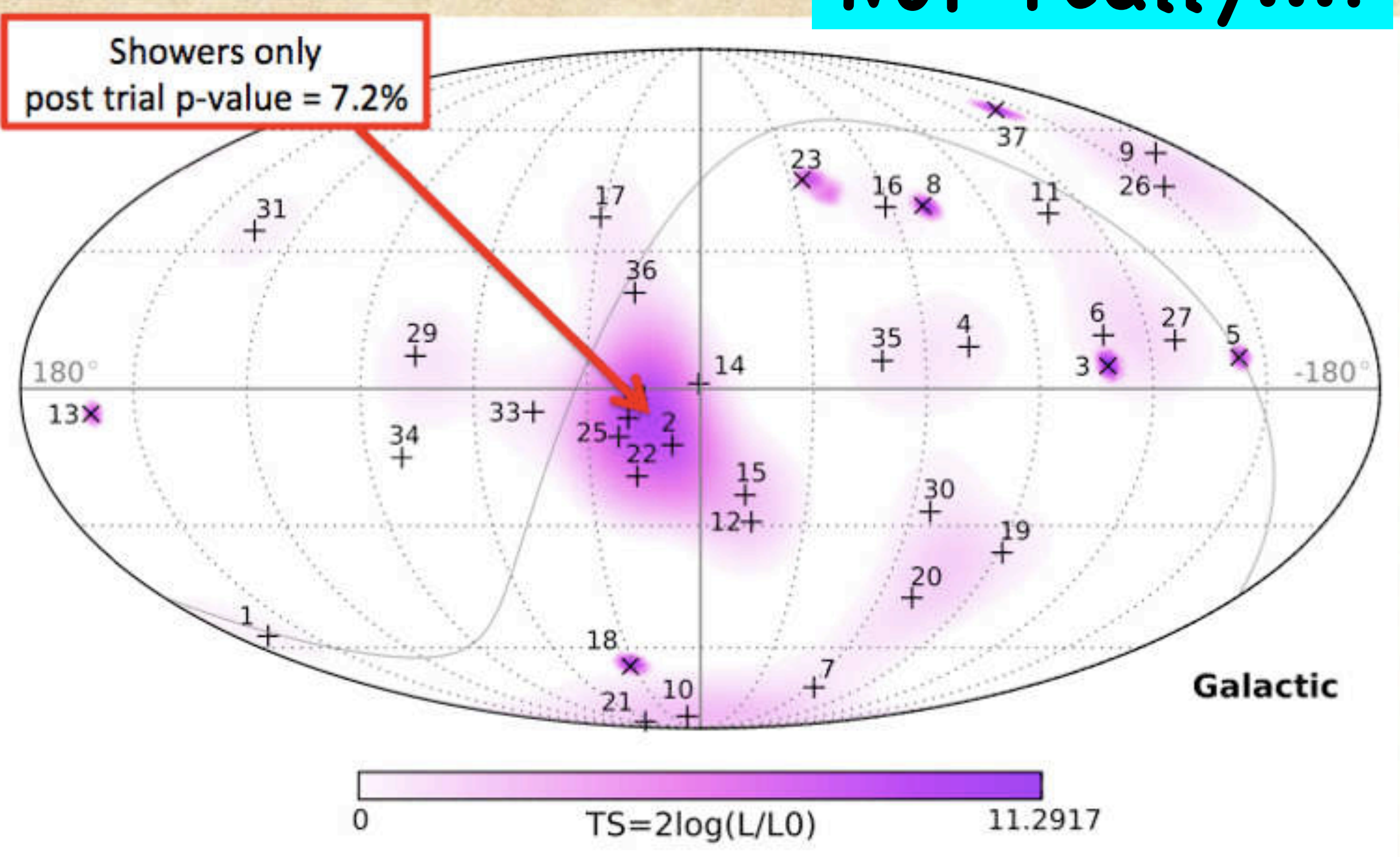




\section{More generally:}

- Could pp sources might explain the PeV nu bkg?

- In a model-indep. way, just assuming spectr. $\mathrm{E}^{-2}$ or $\mathrm{E}^{-2.2}$, the answer is yes

- also suggesting a break@ few PeV

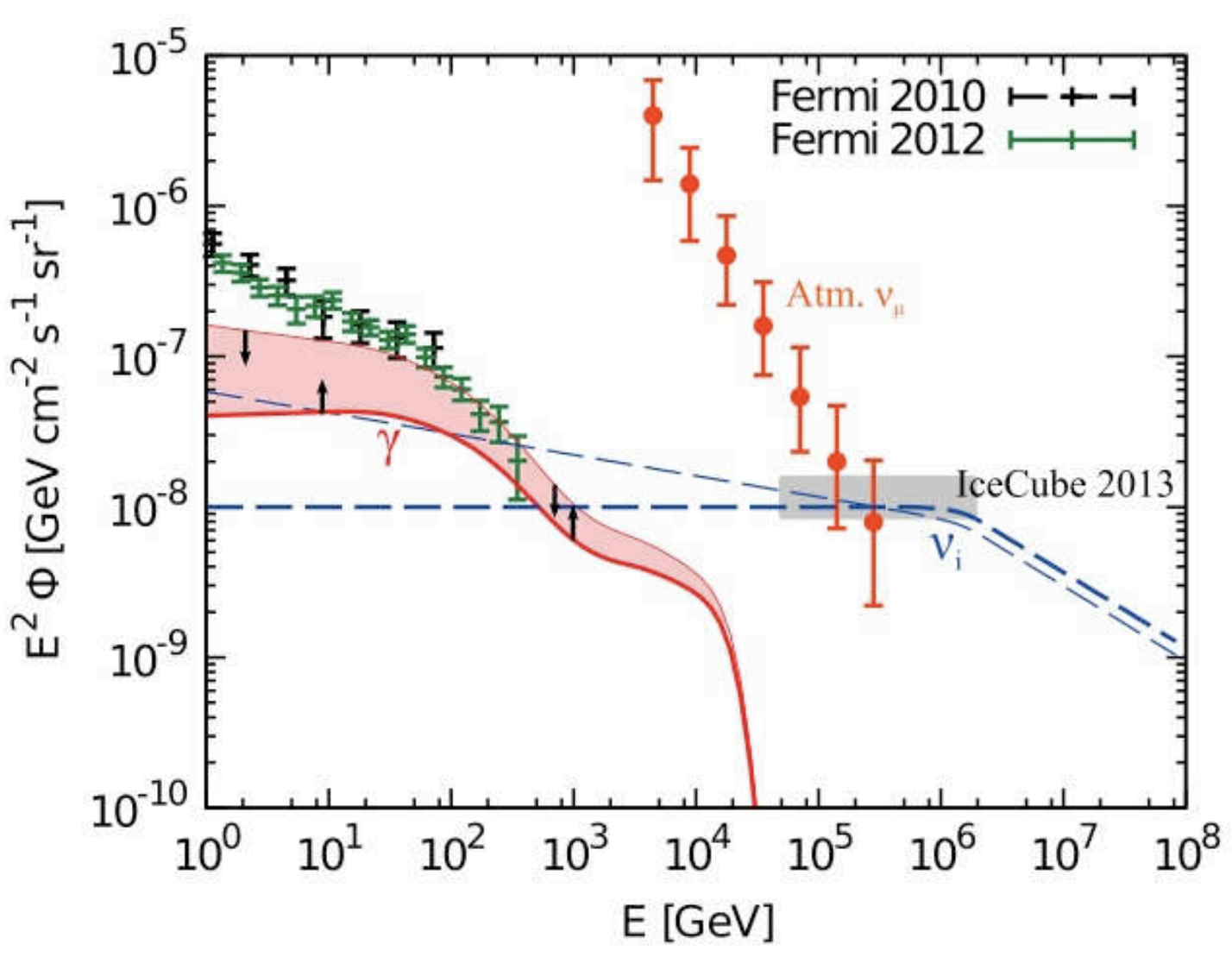




\section{Some specific PP scenarios}

Need: enough CR energy budget, pp efficiency

- Radio Galaxies: CRs I0-100 EeV, escape into cluster IGM, where produce pp nus in the LSS

- IGS (cluster accretion shocks): CRs @ I00PeV, then $\mathbf{p p} \boldsymbol{n u}$ in IGM , \& $\mathrm{t}_{\text {diff }} \sim \mathrm{t}_{\text {inj }} \rightarrow$ sp. break $\checkmark$

- SBGs (starburst gals): may expect higher BISM, both $\mathrm{SNe}, \mathrm{HNe} \rightarrow \mathrm{CRs} @ 100 \mathrm{PeV}, \rightarrow$ pp nus

- HNe (hypernovae) could be candidates (rate?) 


\section{Could these be GRB, or HNe?}

- No "normal" GRB in coincidence with observed PeV events, $\boldsymbol{X}$, (but they could be 'choked' or low Y-luminosity GRBs)

- A small fraction of PeV sources (close to the Galactic Center) might be galactic TeV uni-ID sources - which could be hypernovae (HNe); and CR protons of $10-100 \mathrm{PeV}$, via PP $\rightarrow$ PeV Vs $\mathcal{V}$; BUT: only I/28 best fit - or $3 / 28$ at $90 \% \mathrm{CL}$ (Fox, Kashiyama \& Mészáros, 2013,ApJ, 774:74)

- A plausible guess: the isotropic component may be extragalactic hypernovae (HNe) in ultraluminous IR galaxies (ULIRGs); or

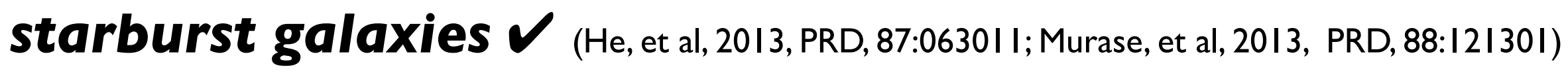

- Note: nu-spectrum must steepen above few PeV, since Glashow resonance [barnue, $\mathrm{e}^{-} \rightarrow(\mathrm{W})$ hadrons] @ 6.3 PeV is not seen $\rightarrow$ corresponding CR spectral slope does not extend to GZK energies $\Rightarrow$ the PeV and GZK CR sources may be different? 


\section{Going above and beyond ...}




\section{Cosmogenic Neutrinos}

$2 \neq \mathrm{CR}$ models

$\downarrow$ same GZK CR fit

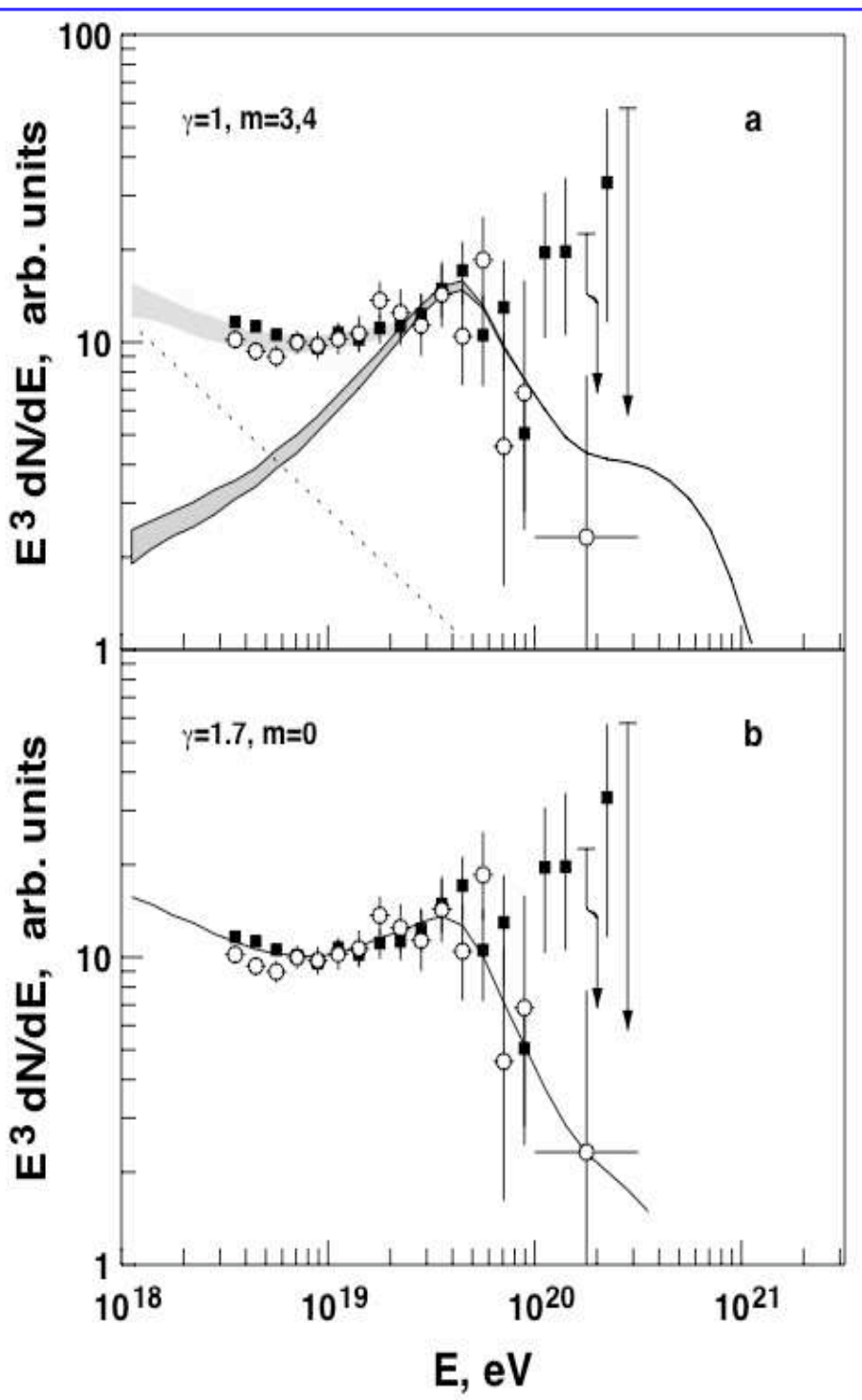

from GZK CRs to GZK vs

get $E_{v} \gtrsim 10^{19} \mathrm{eV}$ ?

But ... lead to $\neq$ GZK $\vee$ flux $\downarrow$

Can infer GZK CR

injection spectrum and/or source cosm. luminosity evolution via their GZK Vs.

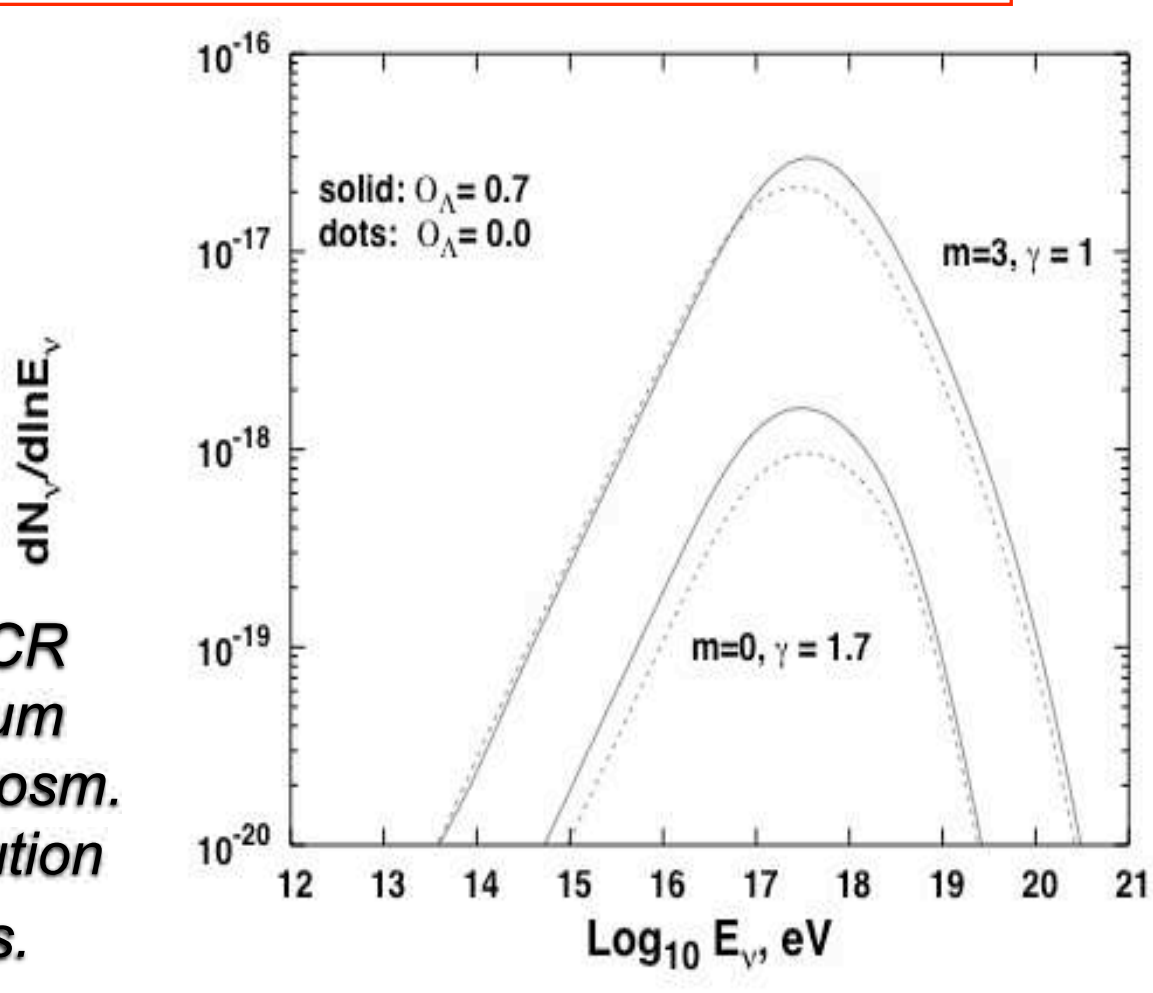

Seckel \& Stanev astroph/050244 


\section{Potential of Cosmogenic $V_{\mathrm{s}}$ for CR Composition}

- If CRs have large fraction of heavies, depending on source distance, photodissociation opt. depth could be $<1 \rightarrow$ only some of them break up into p,n

- Implies smaller fraction contributes to $\pi^{+}$and cosmogenic $\mathrm{V}$ production (Anchordoqui et al 06)

- Cosmogenic $v$ flux vs. CR flux may help resolve discrepancy between Auger $\mathrm{X}_{\max }$ data and apparent correlation with AGN suggesting protons 


\section{ANtarctic Impulsive Transient Antenna}
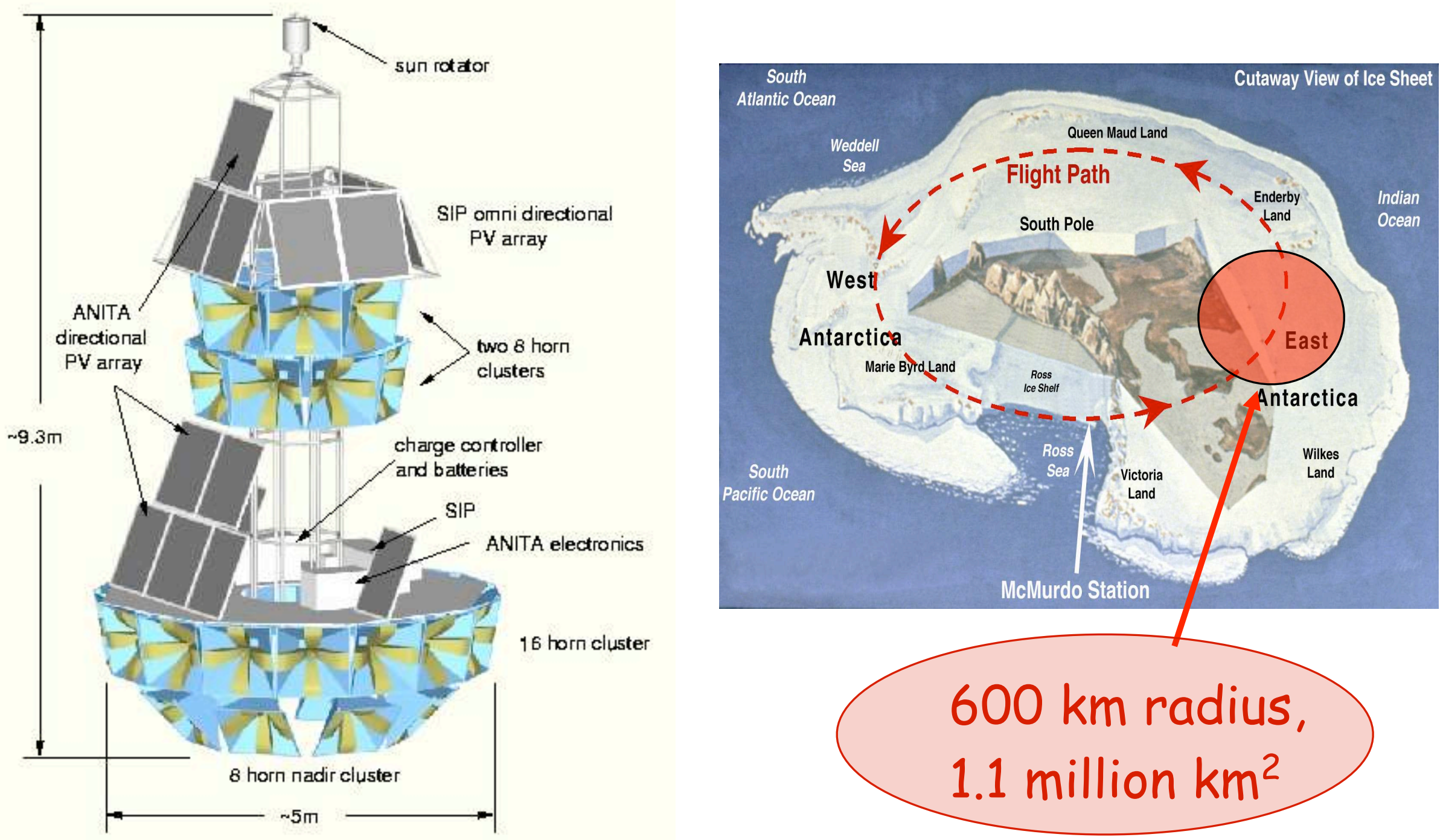

- Launched \& flown 30 days in early 07 - results being analyzed 


\section{ANITA}

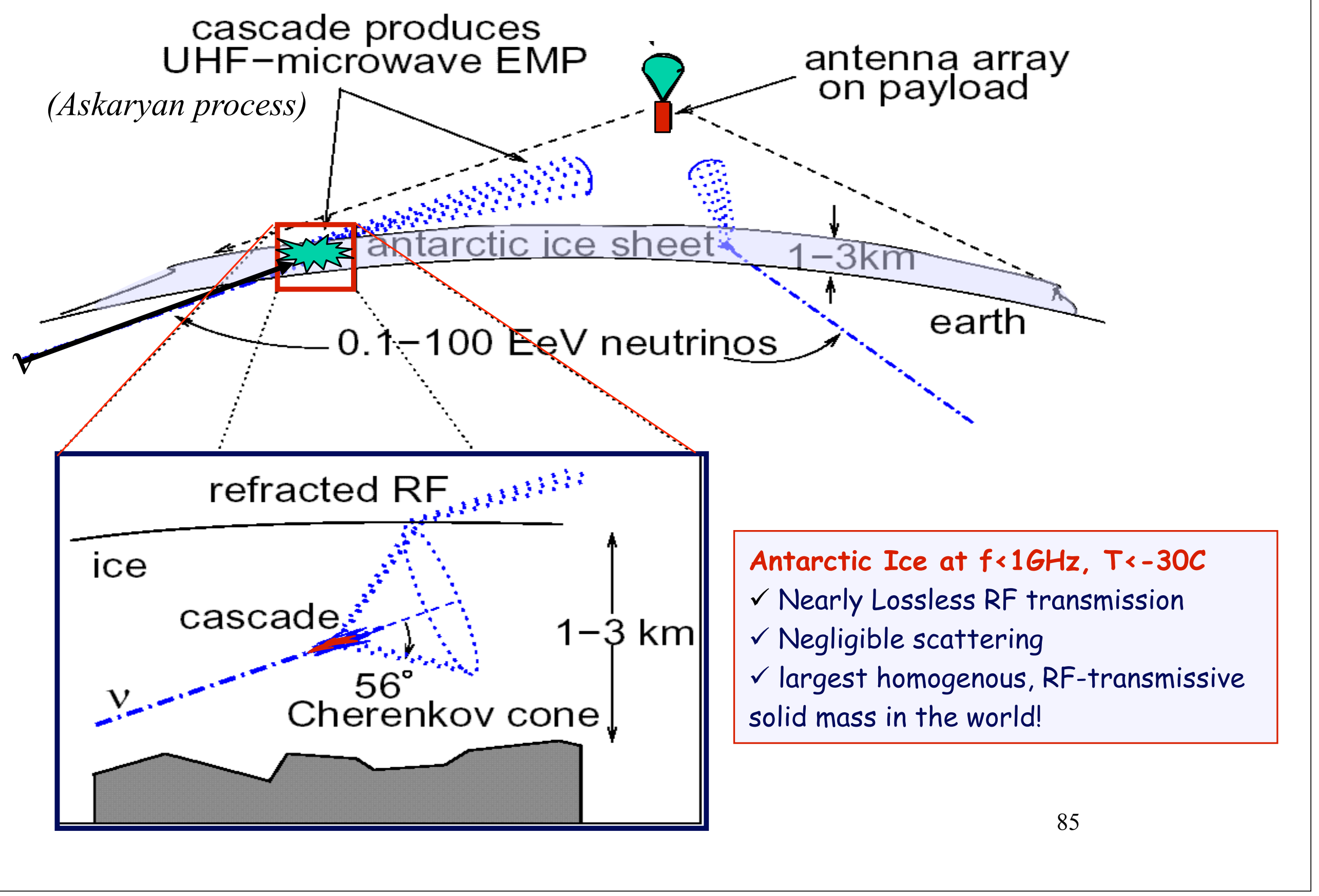




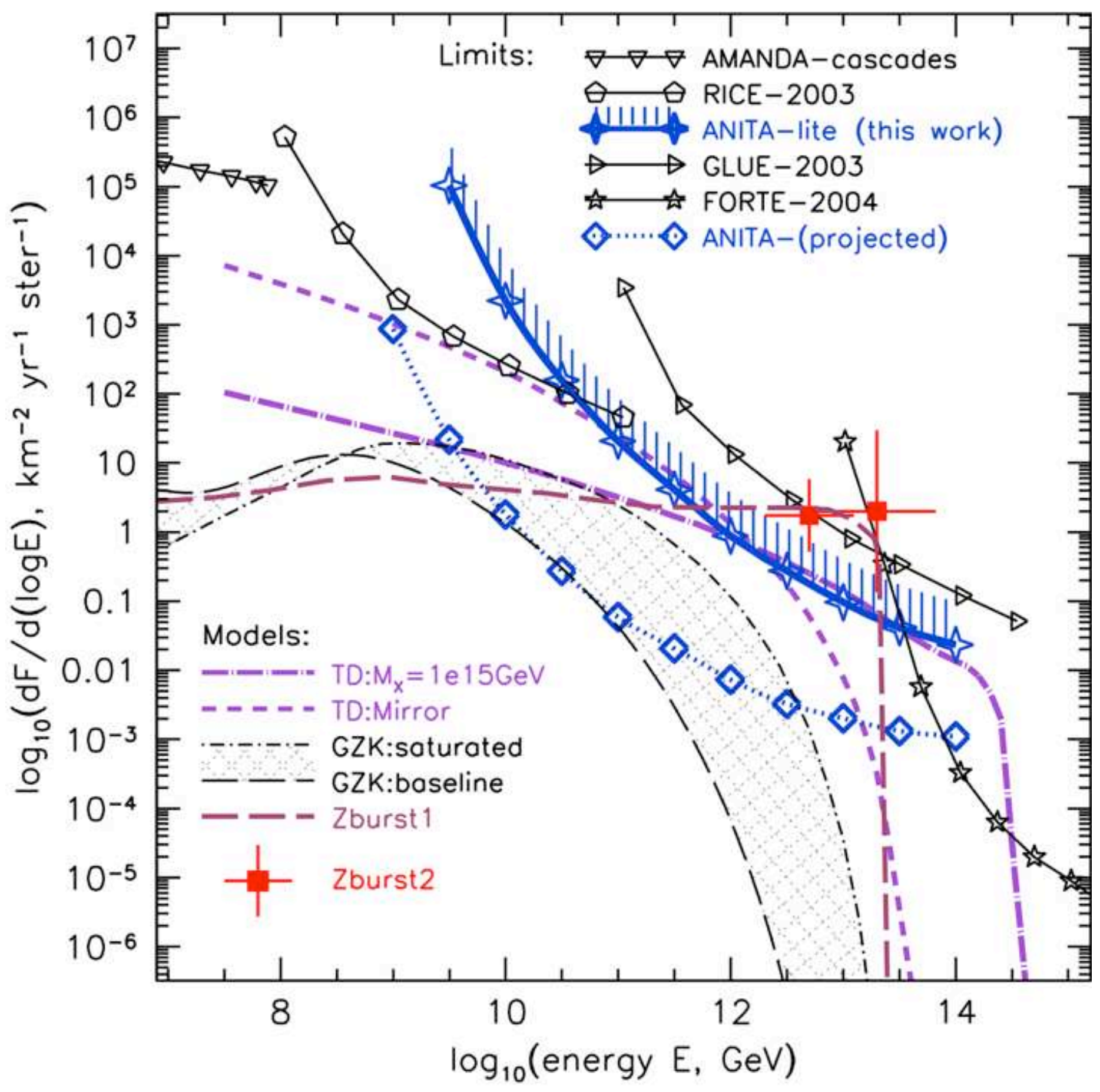

ANITA

GZK

limits

Barwick et al, PRL 96:171101 


\section{Introducing the Askaryan Radio Array (ARA)}

Detect radio emission from Achieve $\mathrm{O}\left(10 \mathrm{~km}^{3}\right)$ detection Use timing and neutrino induced particle volume per station using cascades in Antarctic ice array of antenna clusters polarisation information fo neutrino reconstruction

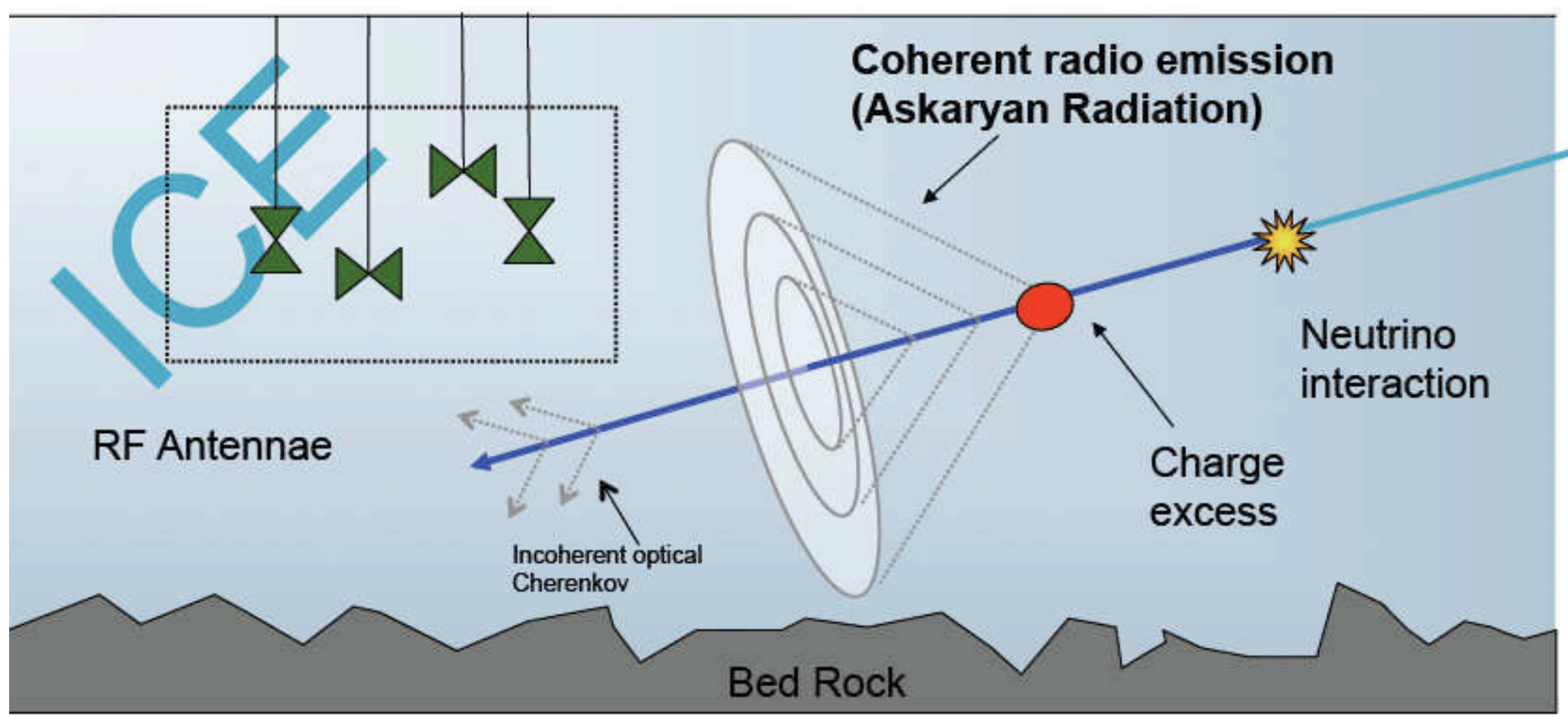

- 100 Gton detector, next to IceCube, sensitive up to $10^{21} \mathrm{eV}_{87}$ (under construction) 


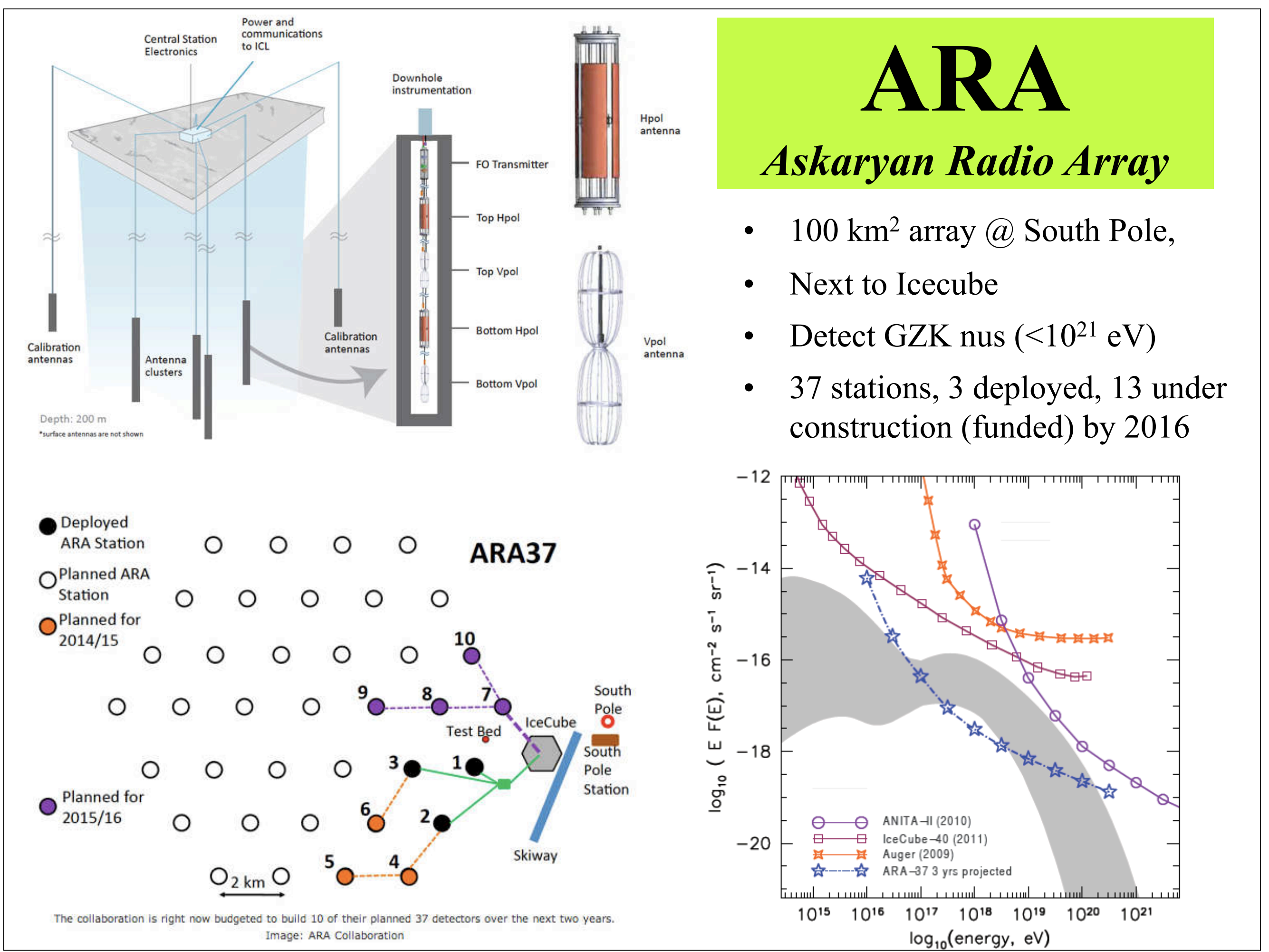




\section{LOFAR : $\quad$ Low Frequency Array}

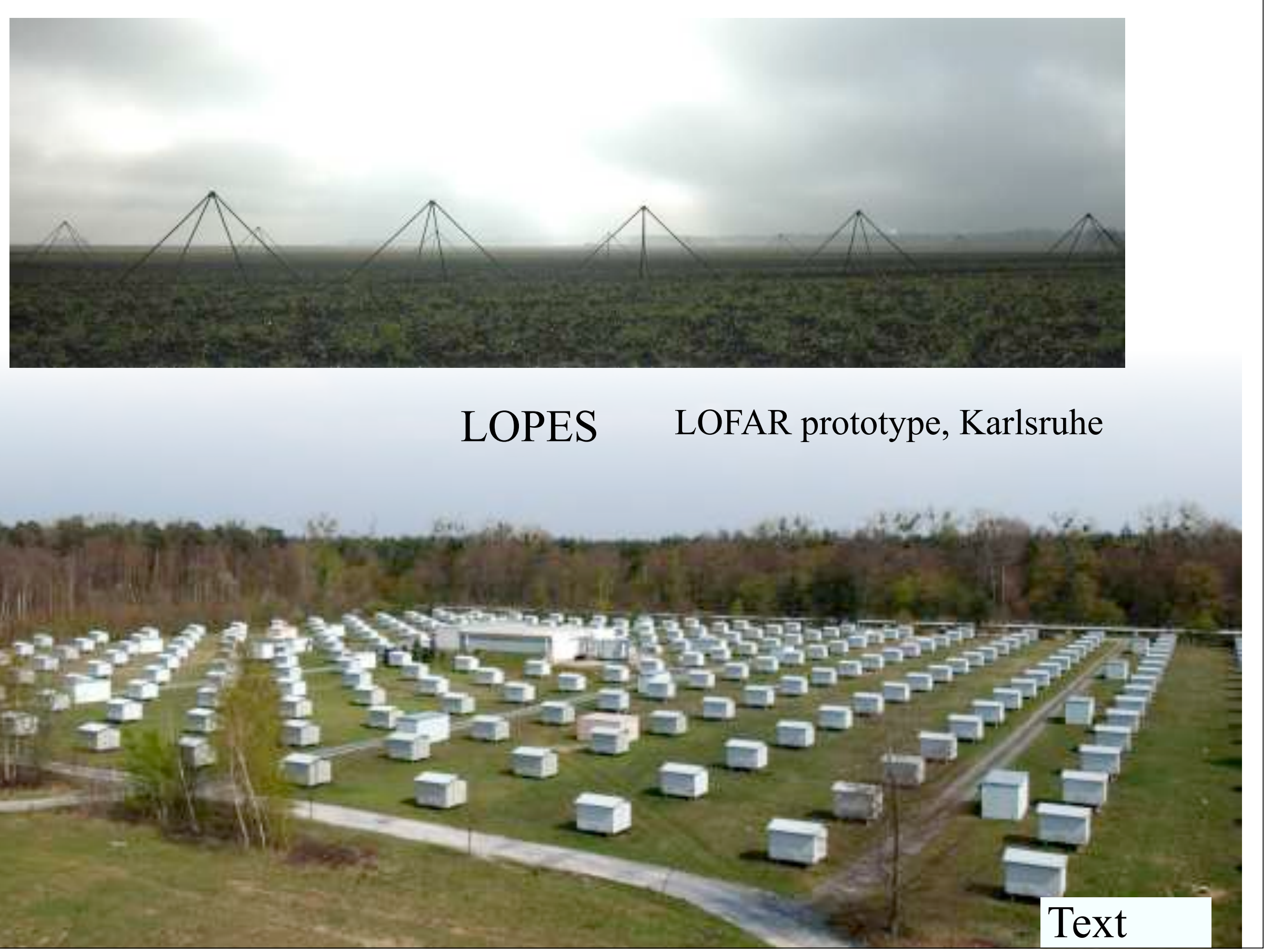




\section{JEM EUSO}

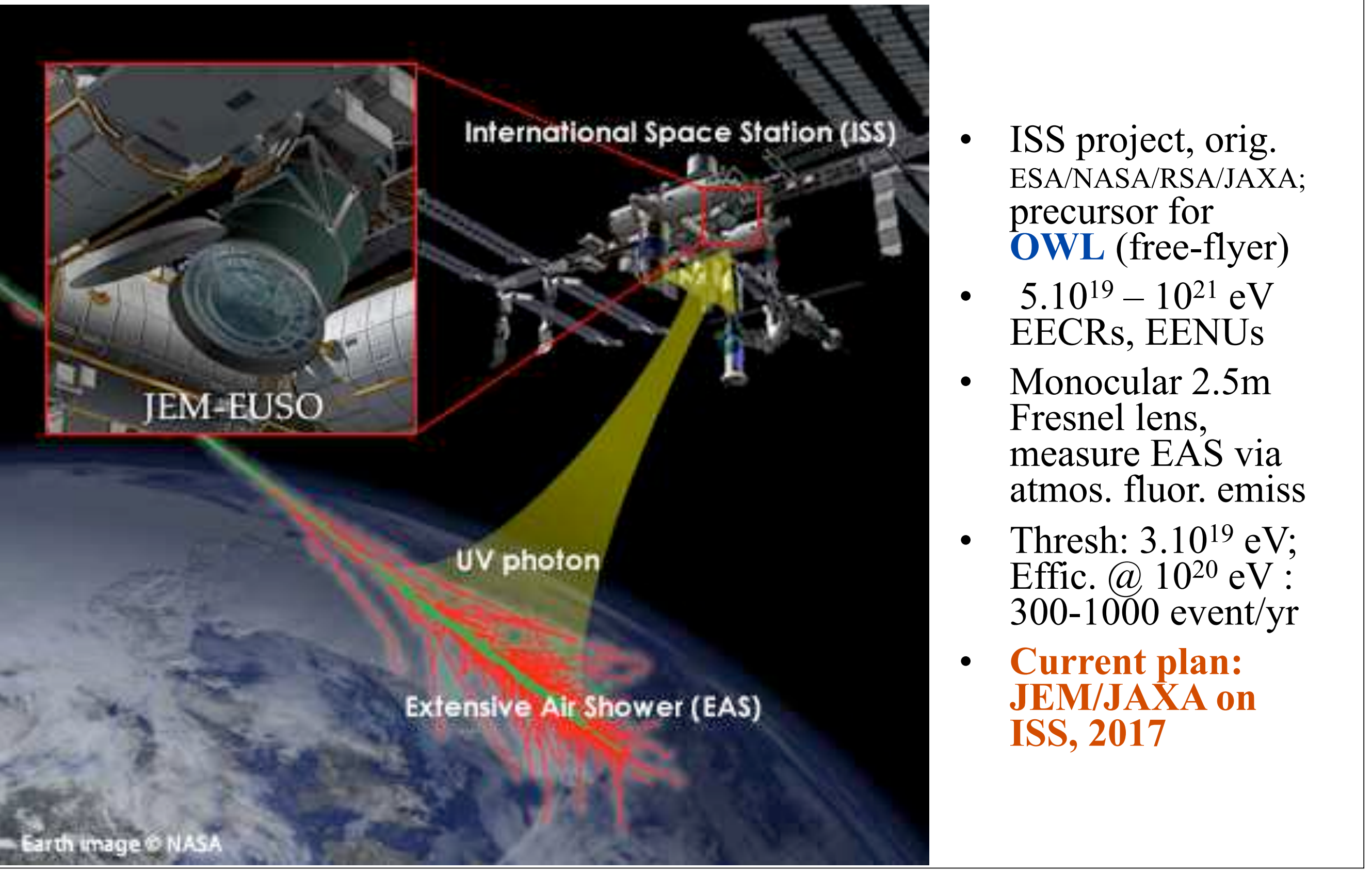




\section{Outlook}

- The sources of UHECR (and of UHENU) are still unknown

- Will learn much about best candidates (GRB, AGN, MGR) from GeV and $\mathrm{TeV}$ photon observations; many with good photon statistics

- Will constrain particle acceleration / shock parameters, compactness of emission region (dimension, mag.field,.)

- UHECR : chemical composition, angular correl.: sources?

- UHE $v$ will allow test of proton content of jets, proton injection fraction, test shock acceleration physics, magn. field

- If UHE v NOT detected in GRB, AGN $\rightarrow$ jets are Poynting dominated!

- Probe $v$ interactions at $\sim$ TeV CM energies

- Constraints on stellar birth \& death rates @ high-z, first structures?

- Cosmogenic nus: probe CR origins, sources 


\section{Outlook:}

- The sources of the UHECR are still unknown..!

- They are almost certainly astrophysical sources (not TD)

- GRB are good candidates, as well as AGNs, HNe, ...

- Will increasingly constrain such possibilities with GeV and TeV photon observations

- Will learn even more if \& when astrophysical UHENUs are observed from any type of identifiable source

- PeV \& sub-PeV neutrinos of astrophysical origin have been (almost certainly) detected

- Current challenge: identify sources of PeV/sub-Pev nus

- Multi-messenger observations, CR composition, clustering will provide important clues 
High Energy Astrophysics

II.

Cosmic Sources of

CRs, Vs, Ys

\& Diffuse Radiation

Péter Mészáros

Pennsylvania State University

NIC20 I 4, Debrecen, 


\section{Who? What?}

\section{(extragalactic)}

- AGNs

- GRBs, $\mathrm{HNe}$

- SFGs, SBGs (star-forming or starburst gals)

- GMSs, GSs (galactic merger or gal. shocks)

- $\Rightarrow$ IGB, INB, ICRB (intergalact. $\gamma$, CR, v bkg)

[ Other (mainly galactic): $\mathrm{SNe}, \mathrm{HNe}, \mathrm{PWNe}$, Binaries ] 
- Photons are the most common and useful cosmic messengers (by far - in number, information content, etc)- so, start with

\section{Extragalactic}

gamma-ray sources 
$100 \mathrm{GeV}$ to $50 \mathrm{TeV}$
HESS VERITAS/MAGIC

Credit TeVCat

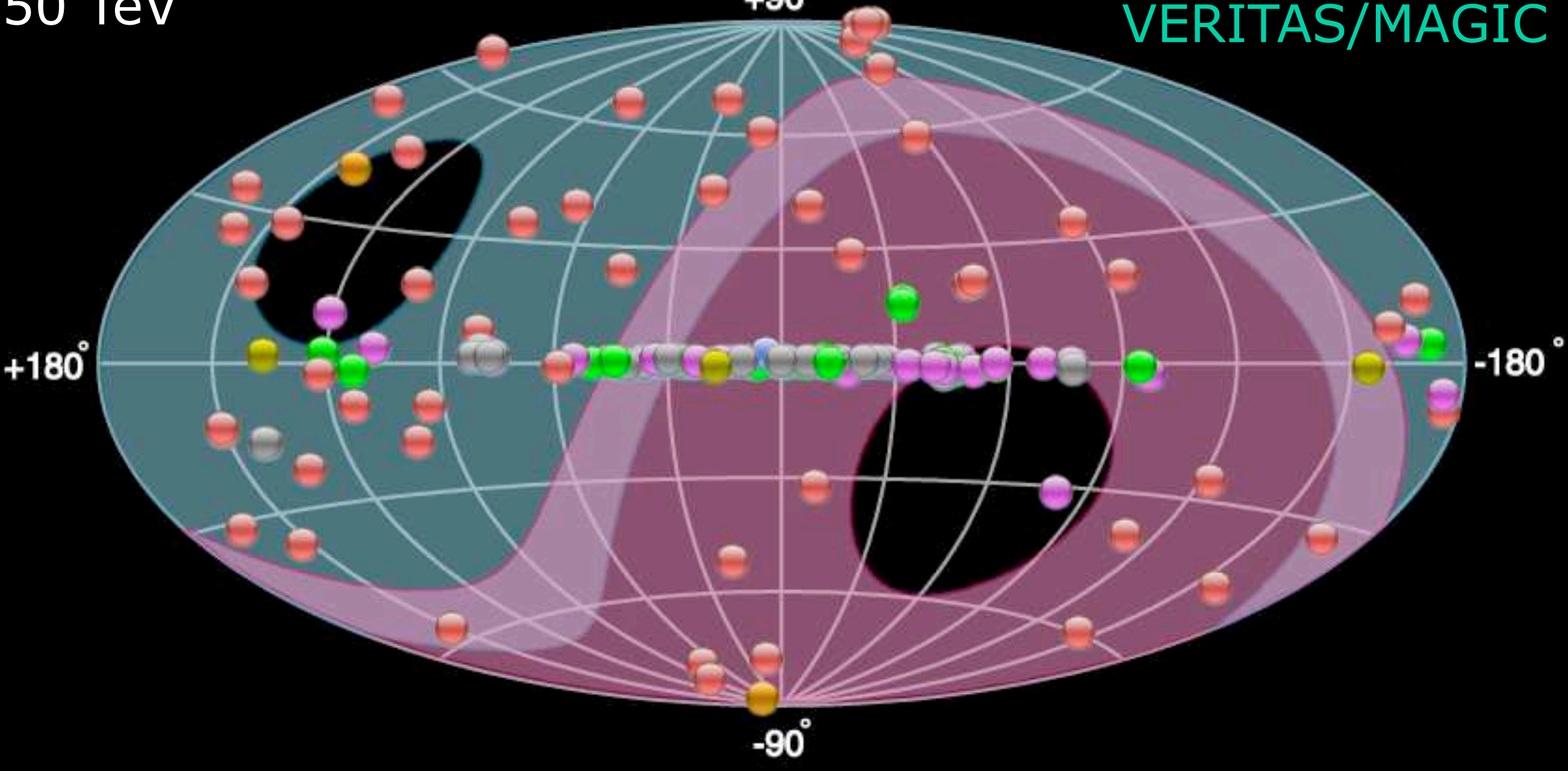

The extragalactic TeV sky is dominated by blazars (mainly BL Lacs) 


\section{AGN as UHE $\gamma$ sources}

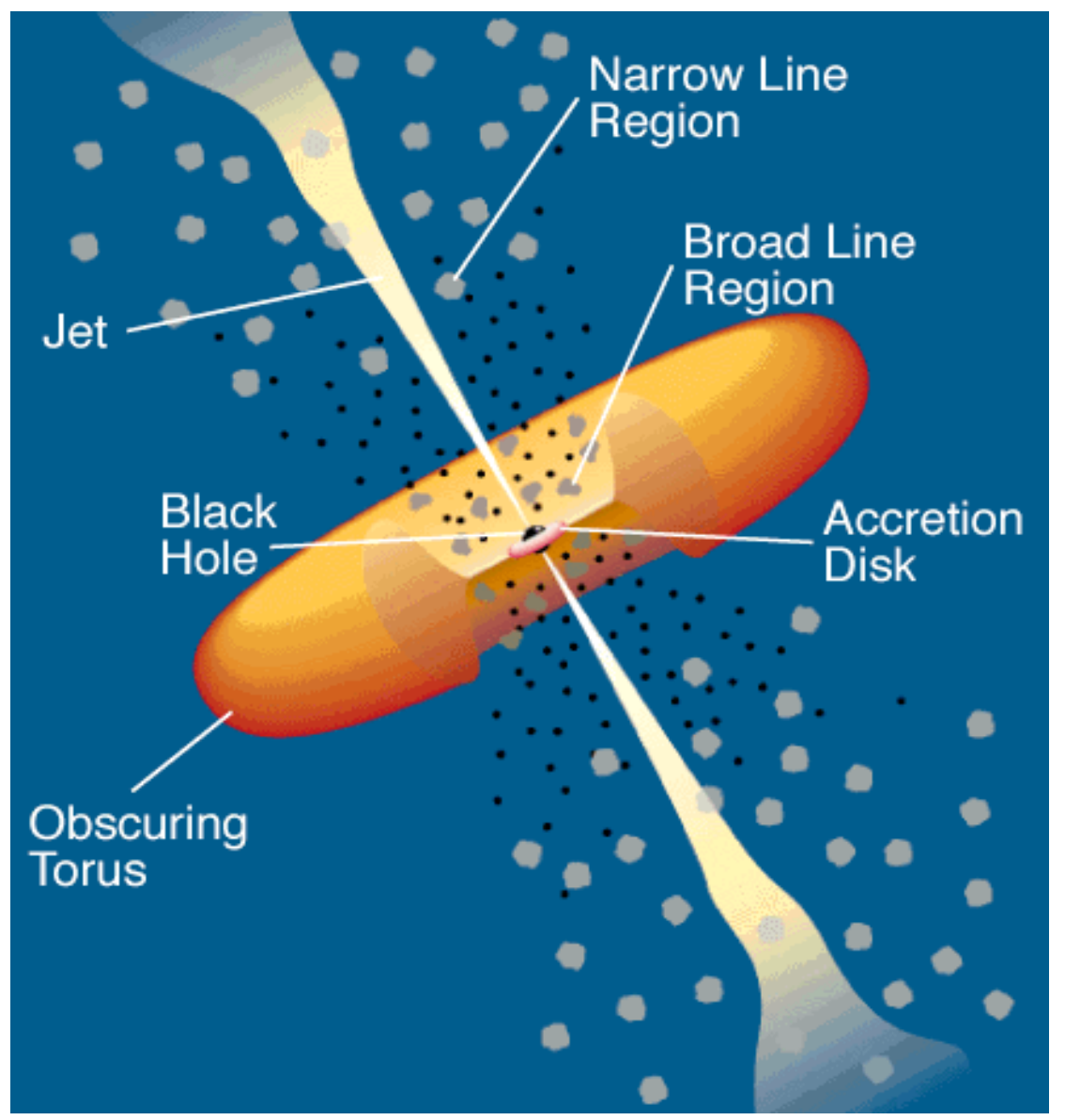

- Massive BH $\left(10^{7}-10^{8} \mathrm{M}_{\text {sun }}\right)$ fed by accretion disk $\rightarrow$ jet

- Lorentz factor $\Gamma_{\mathrm{j}, \text { agn }} \sim 10-30$

- UV target photons from (1) accr. disk, (2) BLR line clouds

- Typical ("leptonic") model: $\mathrm{e}^{ \pm}$accel. in jet shocks, and SSC (sync-self-compton); SEC(sync-exter.compton)

- Typical hadronic model: p accel, in jet shocks, py photomeson interactions, $\rightarrow$ EM cascades 


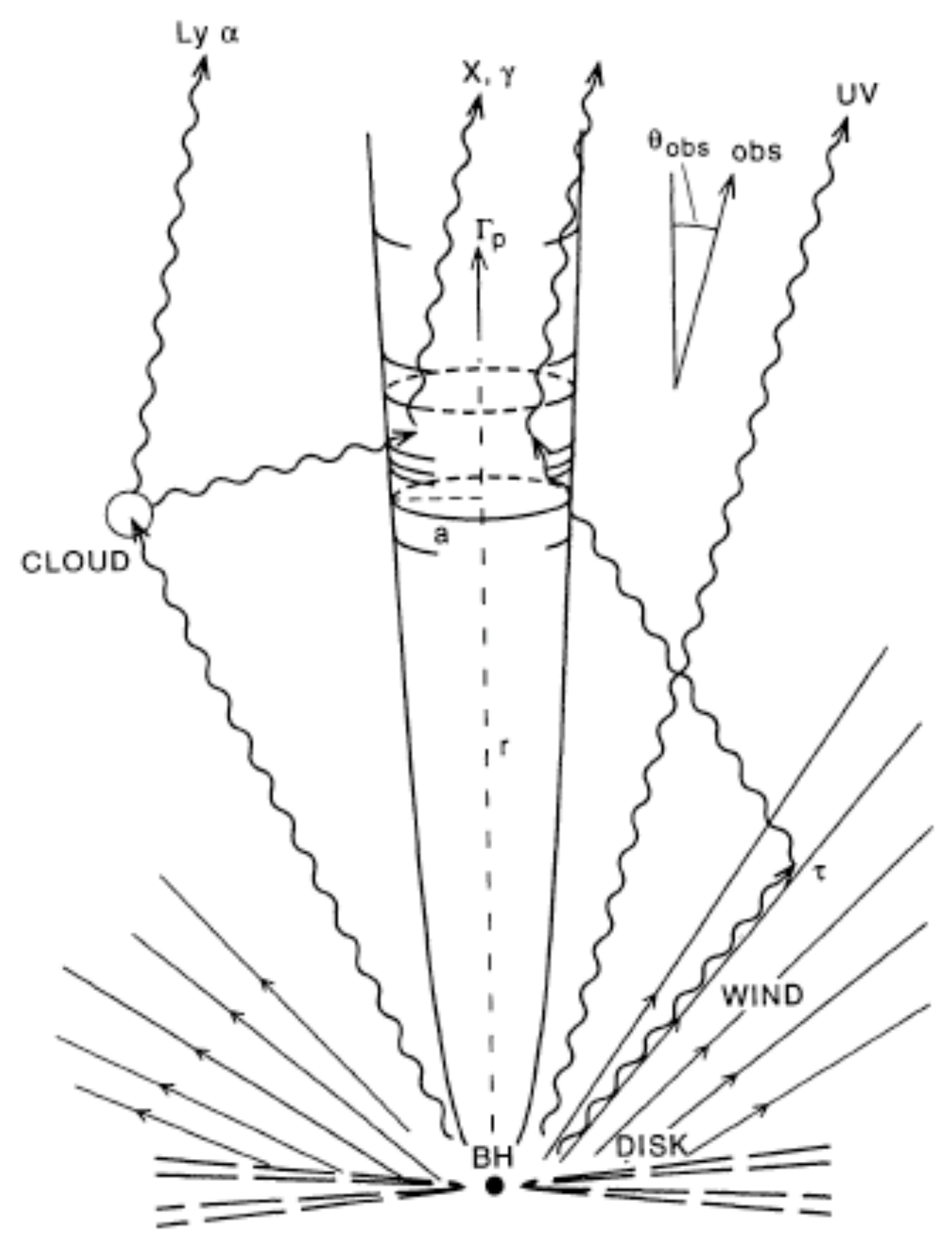

\section{AGN Jet lepto-model}

Sikora et al 

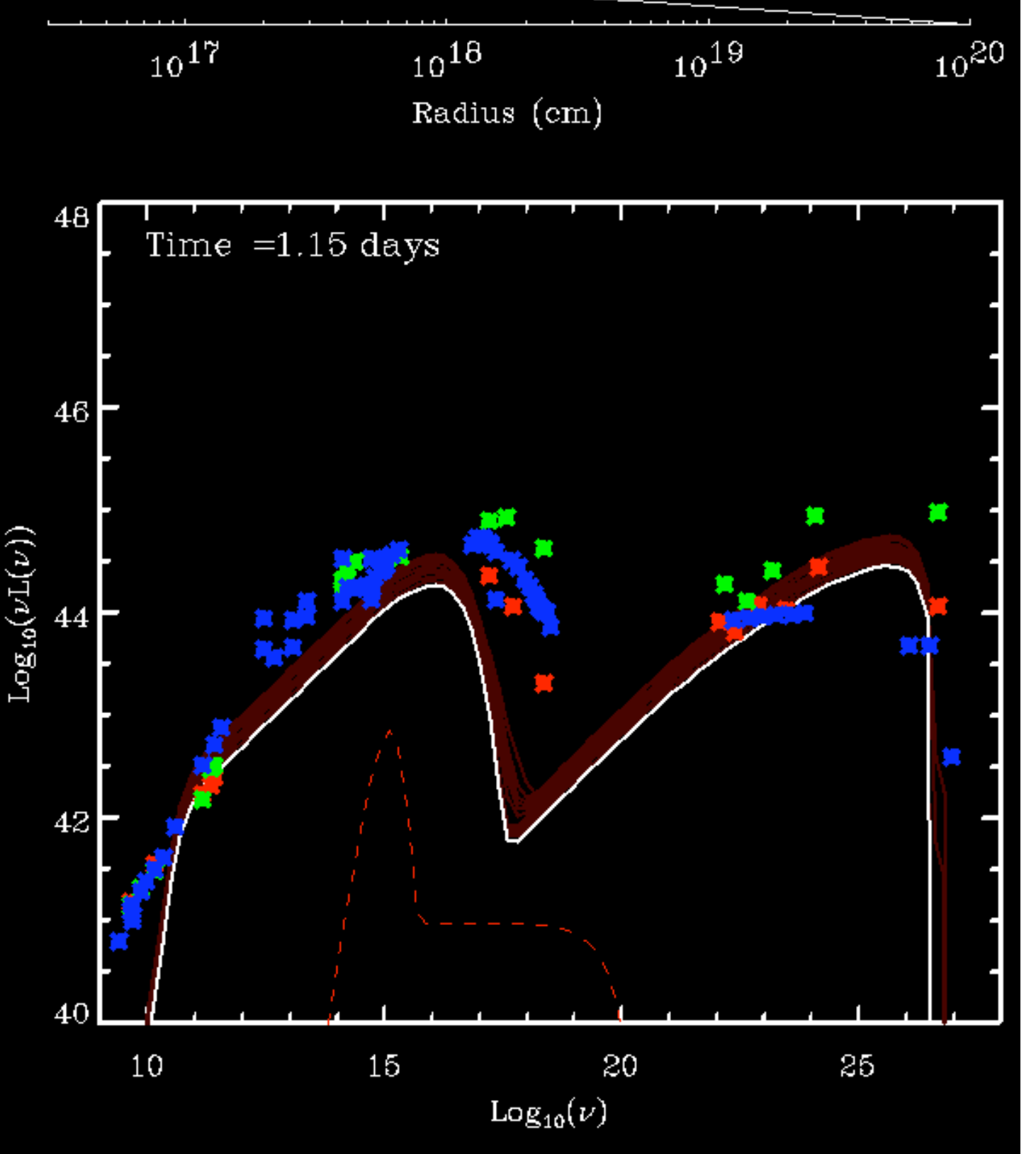

\section{MRK 42I}

Ghisellini et al, Spada et al, leptonic IS model, 2004 


\section{Mrk 42I-leptohadronic}

Dimitrakoudis, Petropoulou,

Mastichiadis 'I4 ApPh 54:61
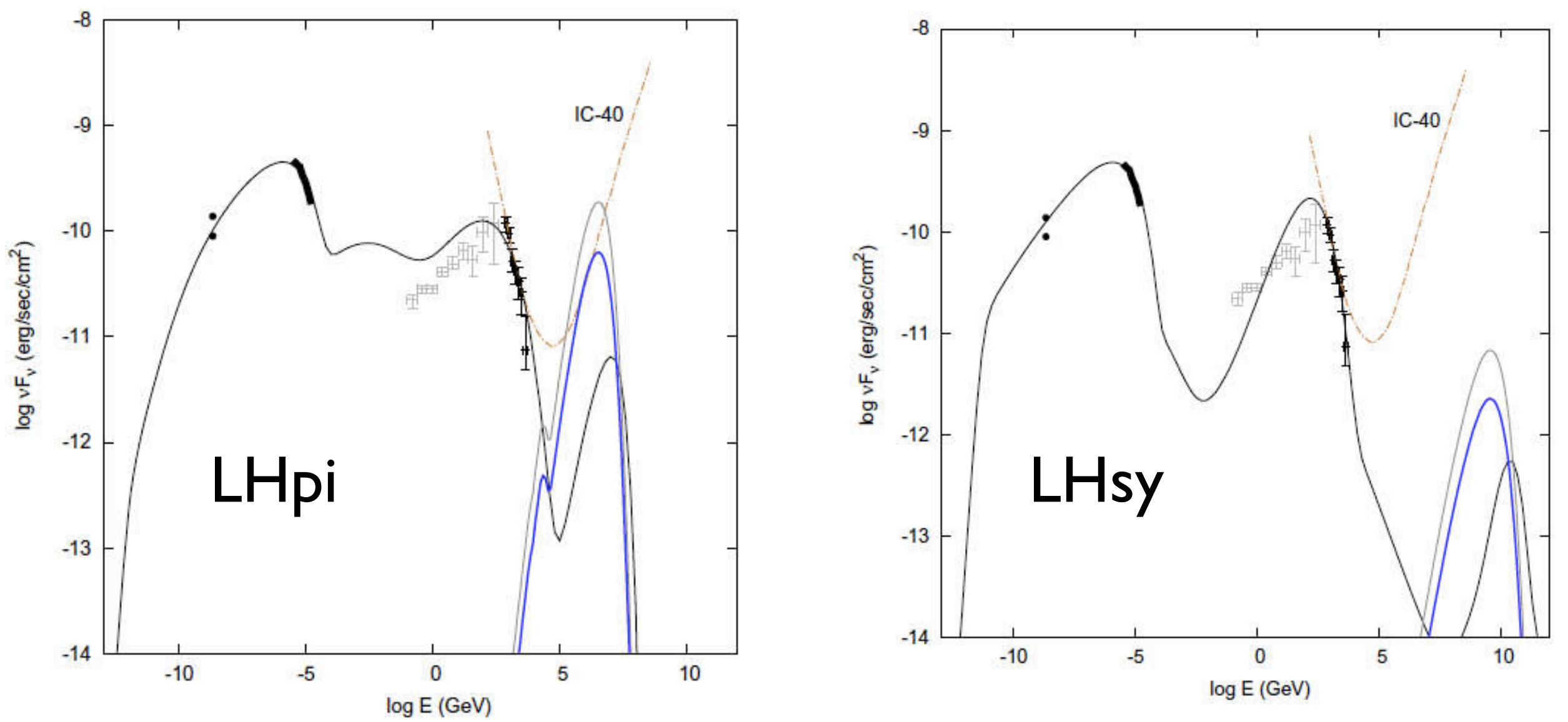

- Two Lepto-Hadronic models: LHpi ( $\gamma$ from pi-decay) and LHsy ( $\gamma$ from p-sync.)

- Use kinetic eqs. for primaries \& decay products, full SOPHIA code for $p, \gamma$

- Fit requires very flat $\Gamma_{\mathrm{p}}, \Gamma_{\mathrm{e}} \sim$ I.2, I.5 (e.g.Niemec-Ostrowski) 


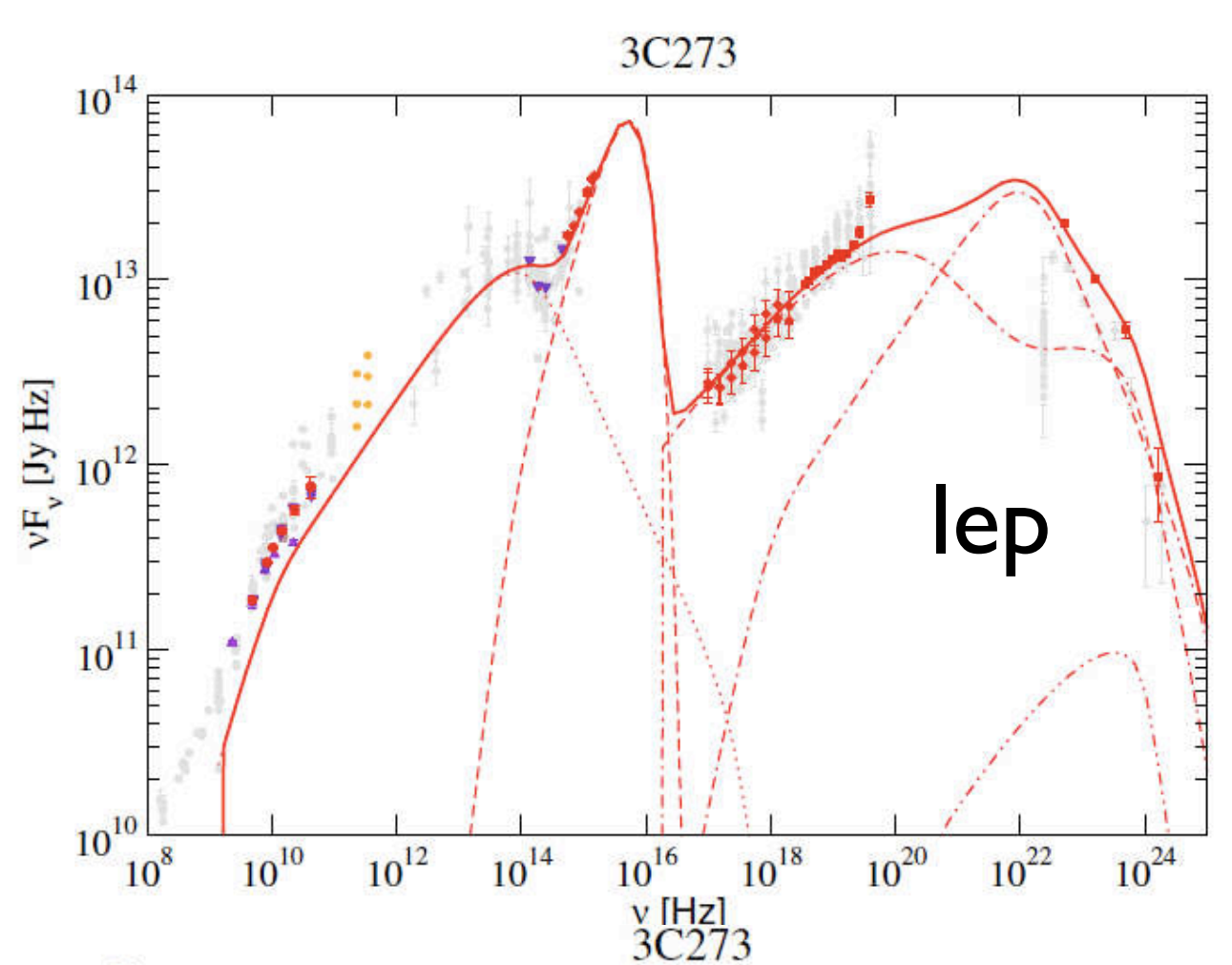

\section{FSRQ 3C273}

Boettcher, Reimer, Sweeney,

Prakash 'I4, apj 768:54

- Compare two models :

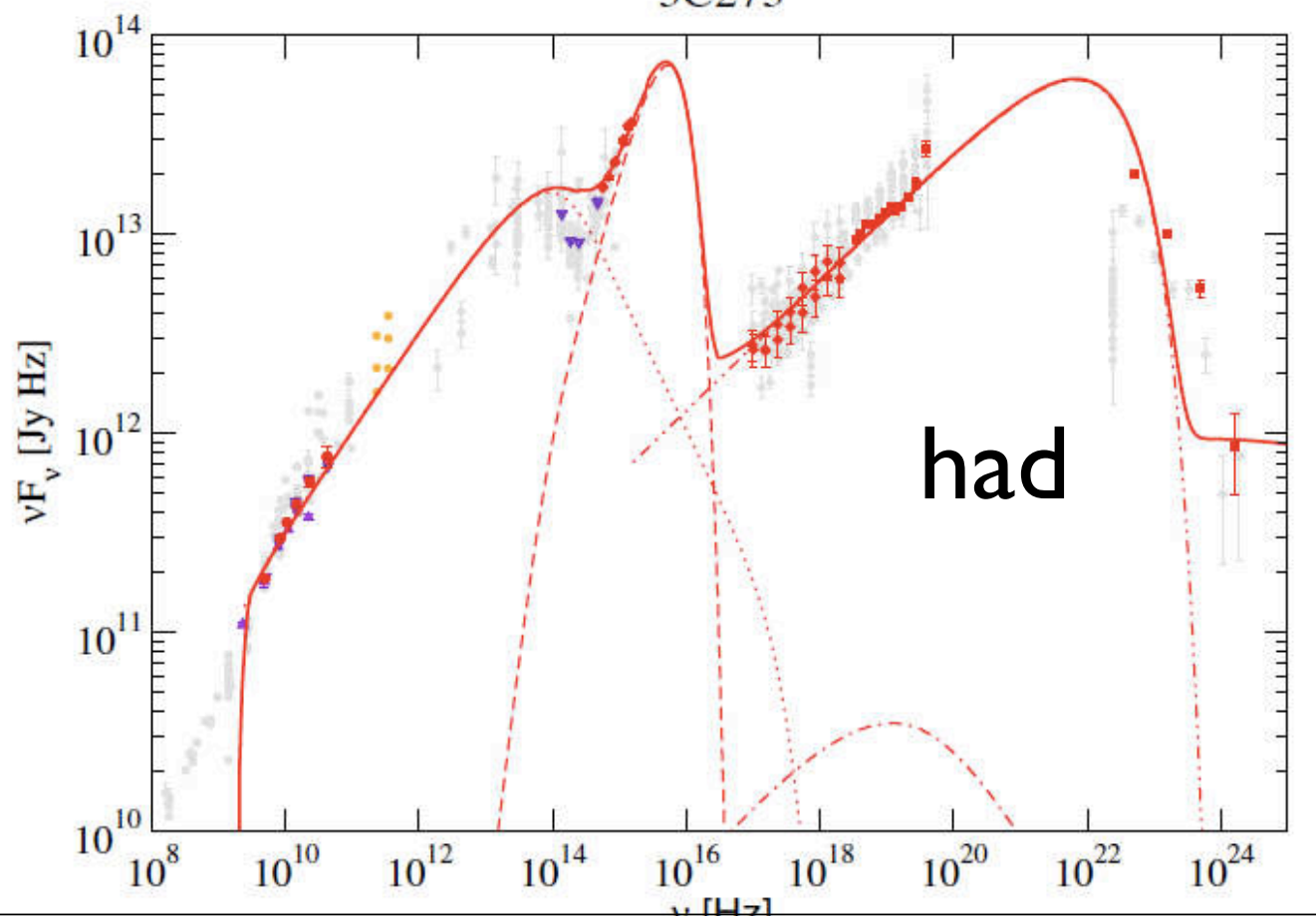

- (I) leptonic SSC, EC

- (2) lepto-hadronic w. semi-analyt. cascades)

- Photon targets from accr. disk, BLR clouds

- Fit 6 FSRQ, 4 LBL, 2 IBL 


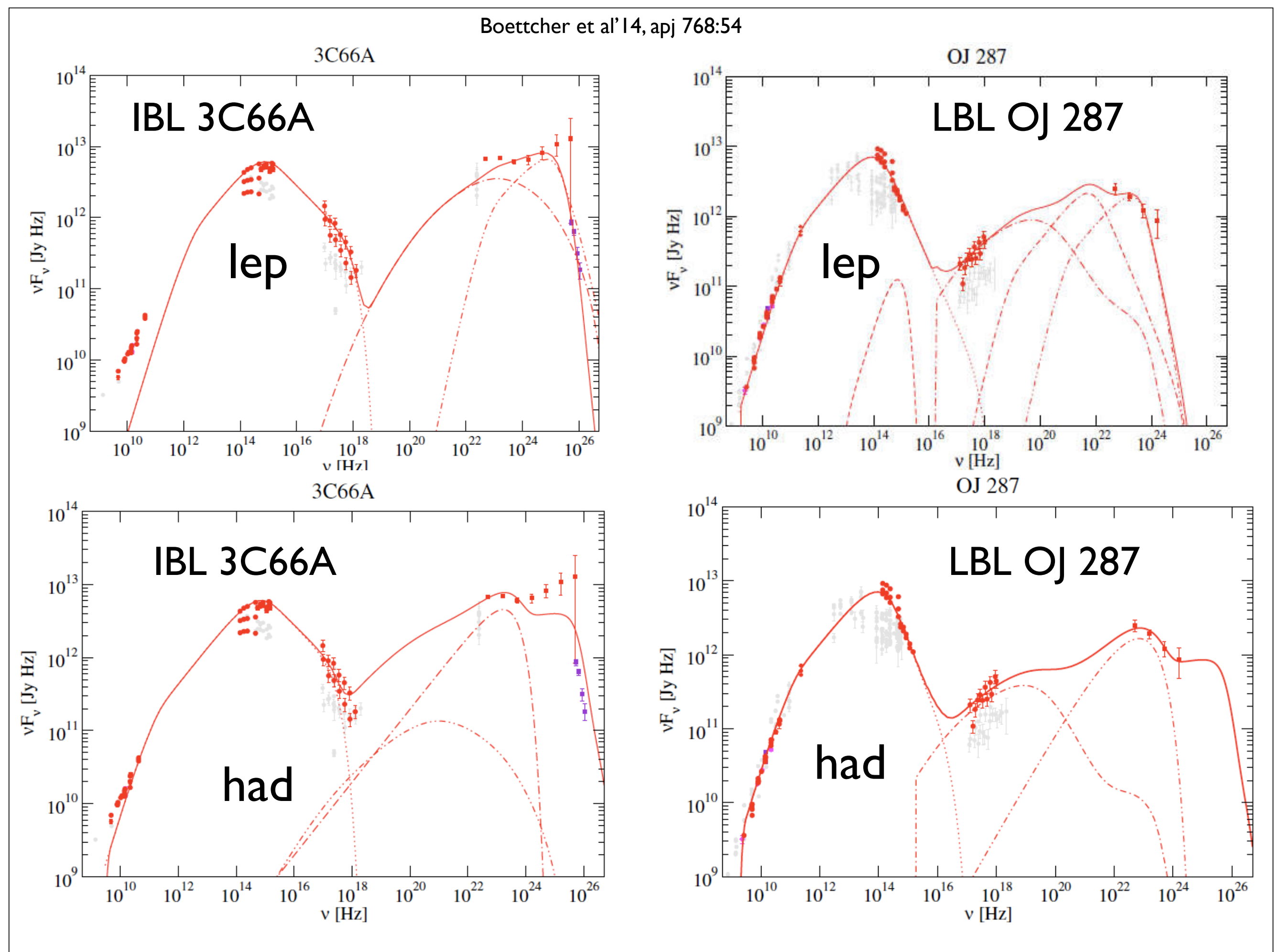




\section{GRBs \& the gamma-ray sky}
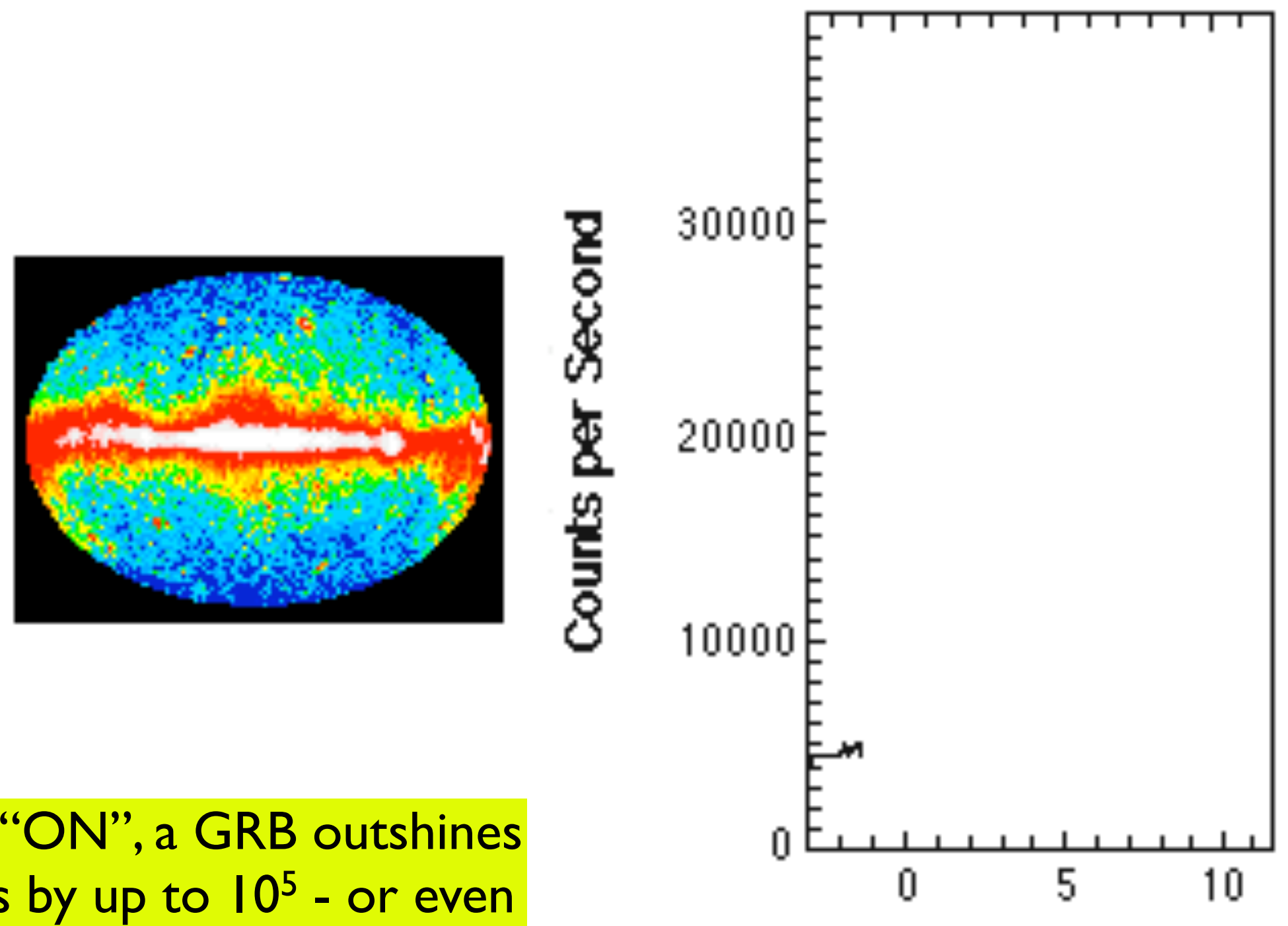

the Sun (in gamma-rays)

Tme in Seconds

When "ON", a GRB outshines blazars by up to $10^{5}$ - or even 


\section{GRB: basic numbers}

- Rate: $~ 1 /$ day inside a Hubble radius

- Distance: $0.1 \leq \mathrm{z} \leq 9.3$ ! $\rightarrow \mathrm{D} \sim 10^{28} \mathrm{~cm}$

- Fluence: $\quad \sim 10^{-4}-10^{-7} \mathrm{erg} / \mathrm{cm}^{2}$

$$
F=\int \text { flux. } d t \sim 1 \mathrm{ph} / \mathrm{cm}^{2} \text { ( } \mathrm{\gamma} \text {-rays !) }
$$

- Energy output: $10^{53}(\Omega / 4 \pi) D^{2}{ }_{28.5} F_{-5}$ erg but, jet: $\left(\Omega_{\mathrm{j}} / 4 \pi\right) \sim 10^{-2} \rightarrow \mathrm{E}_{\mathrm{y}, \mathrm{tot}} \sim 10^{51} \mathrm{erg}$

$\rightarrow E_{y, \text { tot }} \sim L_{\Theta}$ in $10^{10}$ year $\sim L_{\text {gal }}$ in 1 year

- Rate[GRB ( $\gamma$-obs)] $\sim 10^{-6}(2 \pi / \Omega) / y r / g a l ~ \rightarrow 1 /$ day $(z \leq 3)$

but Rate [GRB (uncollimated)] 10-4 /yr/gal, while Rate [SN (core collapse] 10-2/yr/gal, or $10^{7} / \mathrm{yr} \sim 1 / \mathrm{s} \quad(z<3)$ 


\section{GRBs in Cosmological Context}

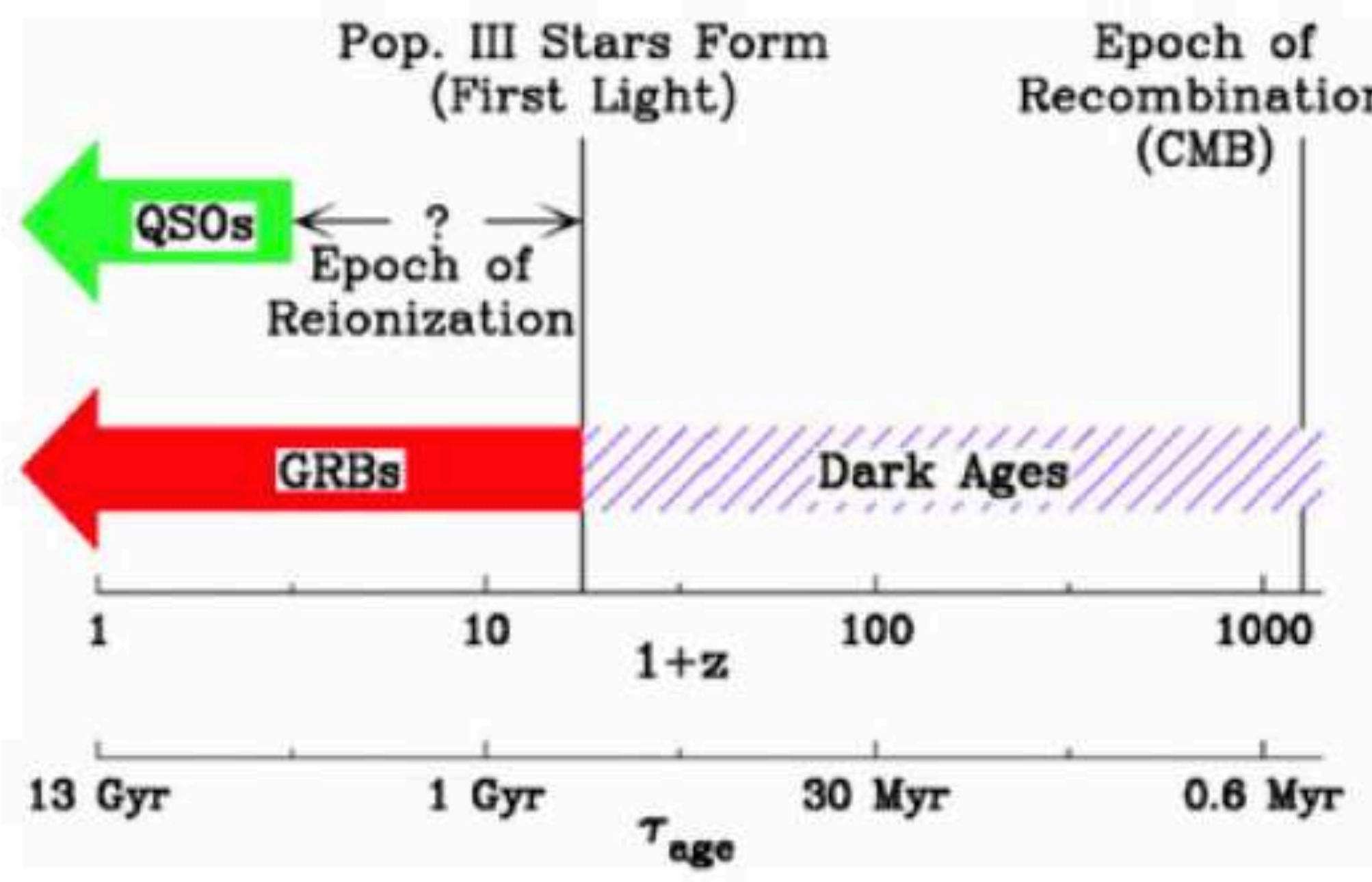




\section{GRB: standard paradigm}

Hyperaccreting Black Holes

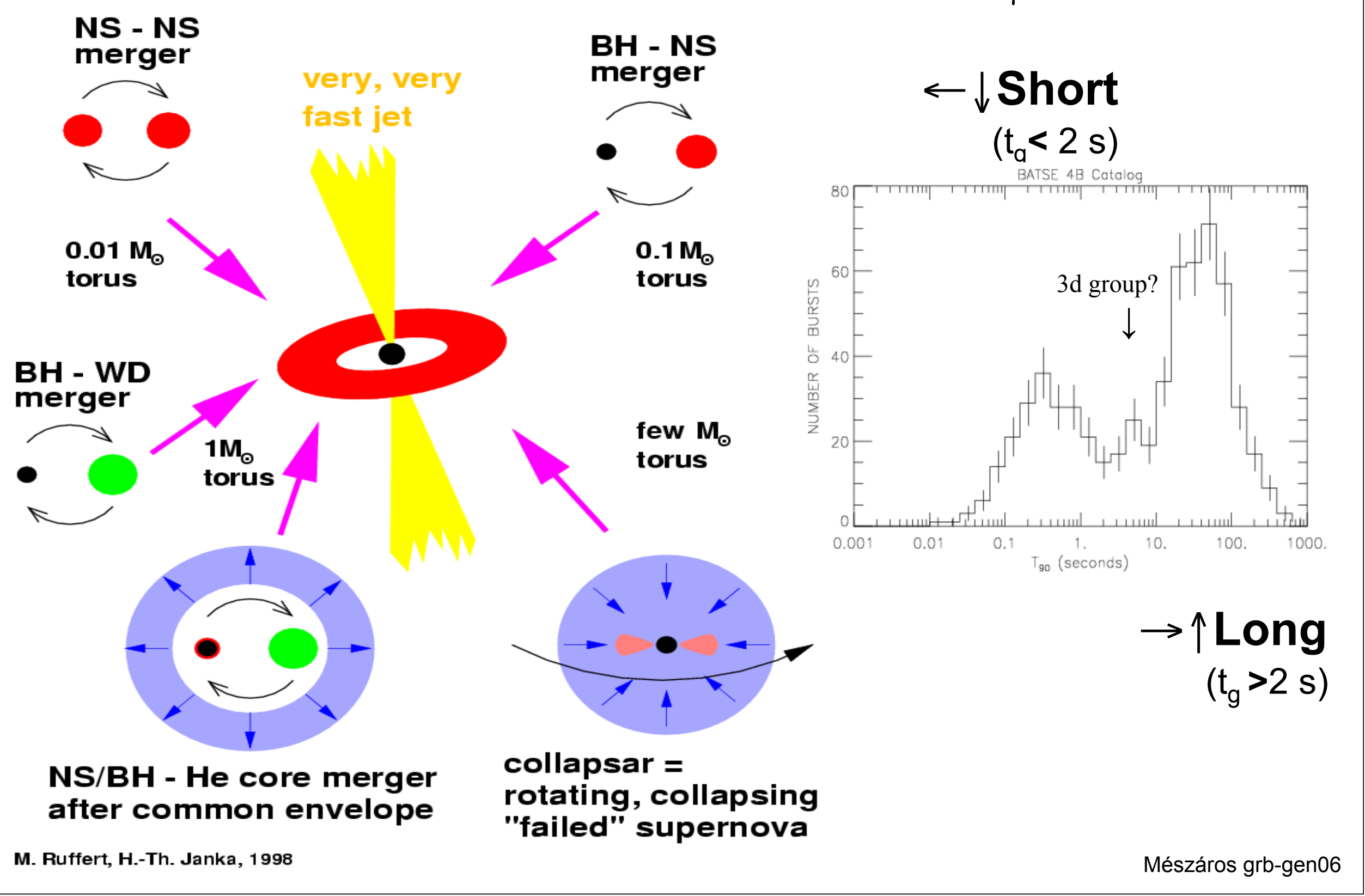




\section{Explosion $\Longrightarrow$ FIREBALL}

- $\mathrm{E}_{\gamma} \sim 10^{51} \Omega_{-2} \mathrm{D}_{28.5}^{2} \mathrm{~F}_{-5}$ erg

- $\mathrm{R}_{0} \sim \mathrm{ct}_{0} \sim 10^{7} \mathrm{t}_{-3} \mathrm{~cm}$

\section{Huge energy in very small volume}

- $\tau_{\gamma \gamma} \sim\left(\mathrm{E}_{\gamma} / \mathrm{R}_{0}^{3} \mathrm{~m}_{\mathrm{e}} \mathrm{c}^{2}\right) \sigma_{\mathrm{T}} \mathrm{R}_{0}>>1$

$\rightarrow$ Fireball: $\boldsymbol{e}^{ \pm}, \gamma, p$ relativistic gas

- $\mathrm{L}_{\gamma} \sim \mathrm{E}_{\gamma} / \mathrm{t}_{0} \gg \mathrm{L}_{\mathrm{Edd}} \rightarrow$ expanding $(\mathrm{v} \sim \mathrm{c})$ fireball

(Cavallo \& Rees, 1978 MN 183:359)

- Observe $\mathrm{E}_{\gamma}>10 \mathrm{GeV}$...but

$\gamma \gamma \rightarrow \mathrm{e}^{ \pm}$, degrade $10 \mathrm{GeV} \rightarrow 0.5 \mathrm{MeV}$ ?

$\mathrm{E}_{\gamma} \mathrm{E}_{\mathrm{t}}>2\left(\mathrm{~m}_{\mathrm{e}} \mathrm{c}^{2}\right)^{2} /(1-\cos \Theta) \sim 4\left(\mathrm{~m}_{\mathrm{e}} \mathrm{c}^{2}\right)^{2} / \Theta^{2}$

\section{Ultrarelativistic flow $\rightarrow \Gamma \geq 0^{-1} \sim 10^{2}$ (bulk Lorentz factor)}

(Fenimore etal 93; Baring \& Harding 94) 


\section{Relativistic Outflows}

- Energy-impulse tensor: $T_{i k}=w_{u_{i}} u_{k}+p g_{i k}$,

$\mathbf{u}^{\mathrm{i}}$ : 4-velocity, $\mathbf{g}_{\mathrm{ik}}=$ metric, $g_{11}=g_{22}=g_{33}=-g_{00}=1$, others 0 ;

ultra-rel. enthalpy: $w=4 p \propto n^{4 / 3} ; w, p, n$ : in comoving-frame

- 1-D motion : $u^{i}=(y, u, 0,0)$, where $\mathbf{u}=\Gamma(\mathbf{v} / \mathbf{c})$,

$\mathbf{v}=3$-velocity, $\quad \mathbf{A}=$ outflow channel cross section :

- Impulse flux energy flux particle number flux

- Isentropic flow : L, J constant $\rightarrow$

$\mathbf{w} \boldsymbol{\Gamma} / \mathbf{n}=$ constant (relativistic Bernoulli equation);

for ultra-rel. equ. of state $p \propto \mathbf{W} \propto n^{4 / 3}$, and cross section $A \propto r^{2}$

$\rightarrow \mathrm{n} \propto 1 / \mathrm{r}^{2} \Gamma$
$\rightarrow \Gamma \propto \mathrm{r}$

- But, eventually saturates, $\Gamma \rightarrow \mathrm{E}_{\mathrm{j}} / \mathrm{M}_{\mathrm{j}} \mathrm{c}^{2} \sim$ constant comoving density drops

"bulk" Lorentz factor initially grows with $r$.

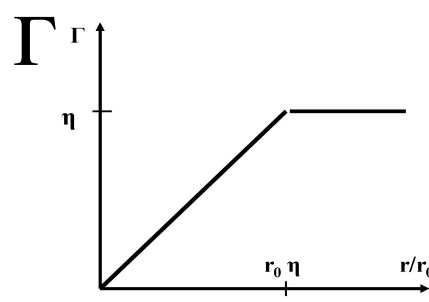

$\Gamma \propto \boldsymbol{r} \rightarrow \Gamma \sim$ const. 


\section{Also expect:}

\section{Internal \& External Shocks} in optically thin medium :

\section{LONG-TERM BEHAVIOR}

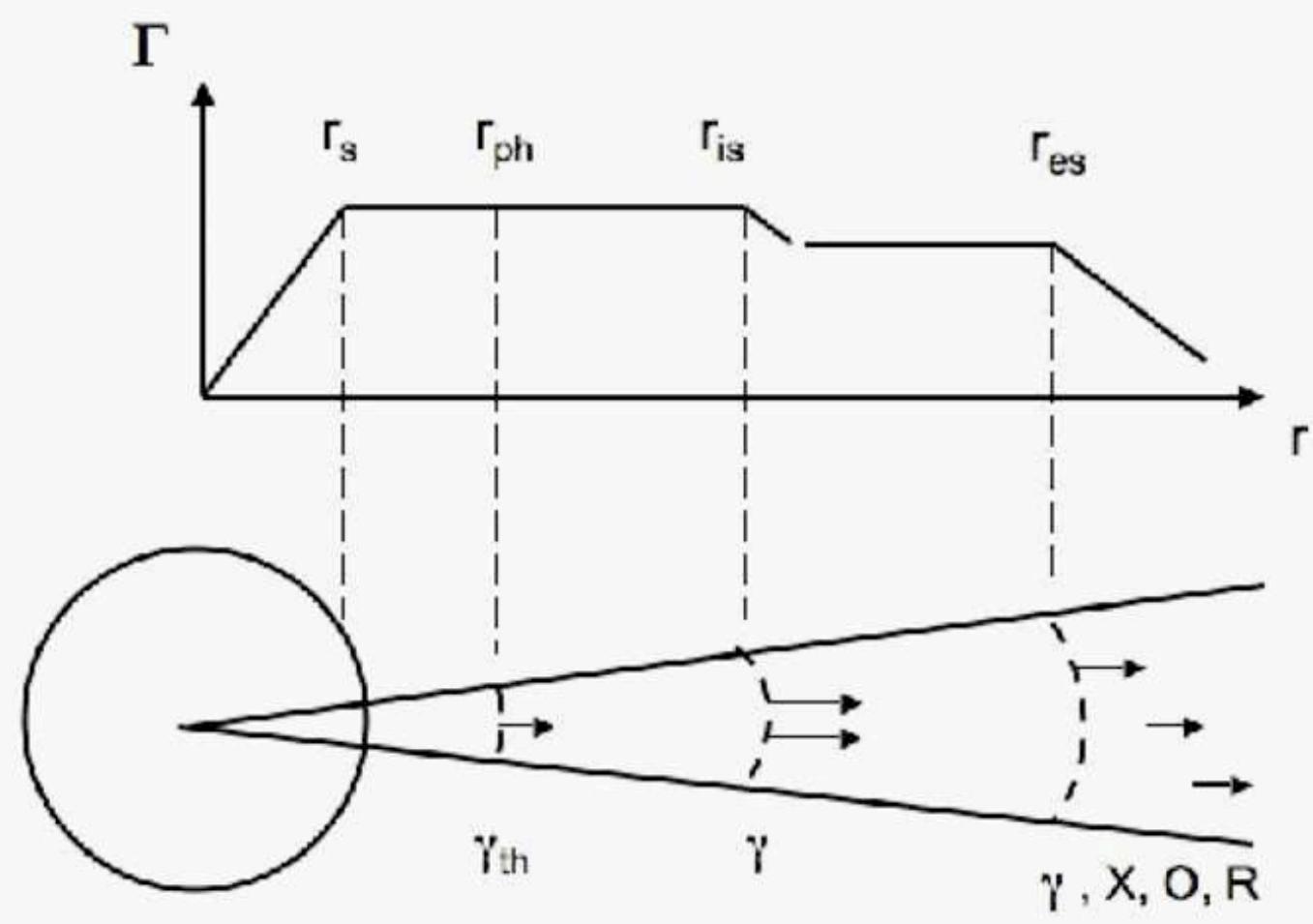

- Internal shocks (or other, e.g. magnetic dissipation) at radius $r_{i} \sim 10^{12} \mathrm{~cm}$

$$
\rightarrow \text { Y-rays (burst, } t_{\gamma} \sim \mathrm{sec} \text { ) }
$$

- $\quad$ External shocks at $r_{e} \sim 10^{16} \mathrm{~cm}$; progressively decelerate, get weaker and redder in time (Rees \& Meszaros 92)

- Decreasing Doppler boost: $\rightarrow$ roughly, expect radio @ 1 week , optical @ 1 day (Paczynski, \& Rhoads 93, Katz 94)

- PREDICTION :

Full quantitative theory of:

- External forward shock spectrum softens in time:

$\mathrm{X}$-ray, optical, radio ... $\rightarrow$ long fading afterglow (t $\sim$ min, hr, day, month)

- External reverse shock (less relativistic, cooler, denser):

\section{Prompt Optical $\rightarrow$ quick fading}

$(\mathrm{t} \sim \operatorname{mins})$

(Meszaros \& Rees 1997 ApJ 476,232) 


\section{Fireball Shock Model of GRBs}

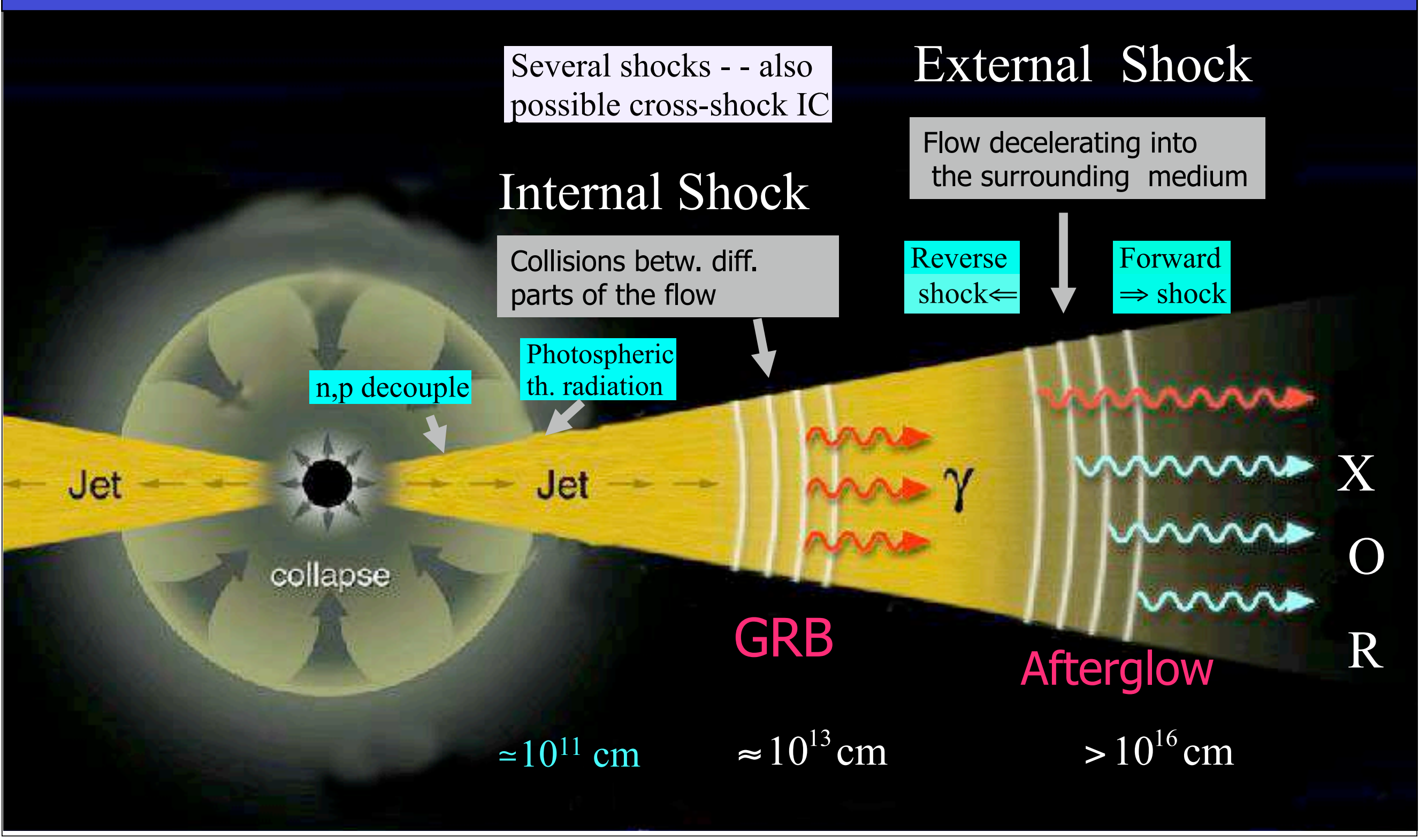




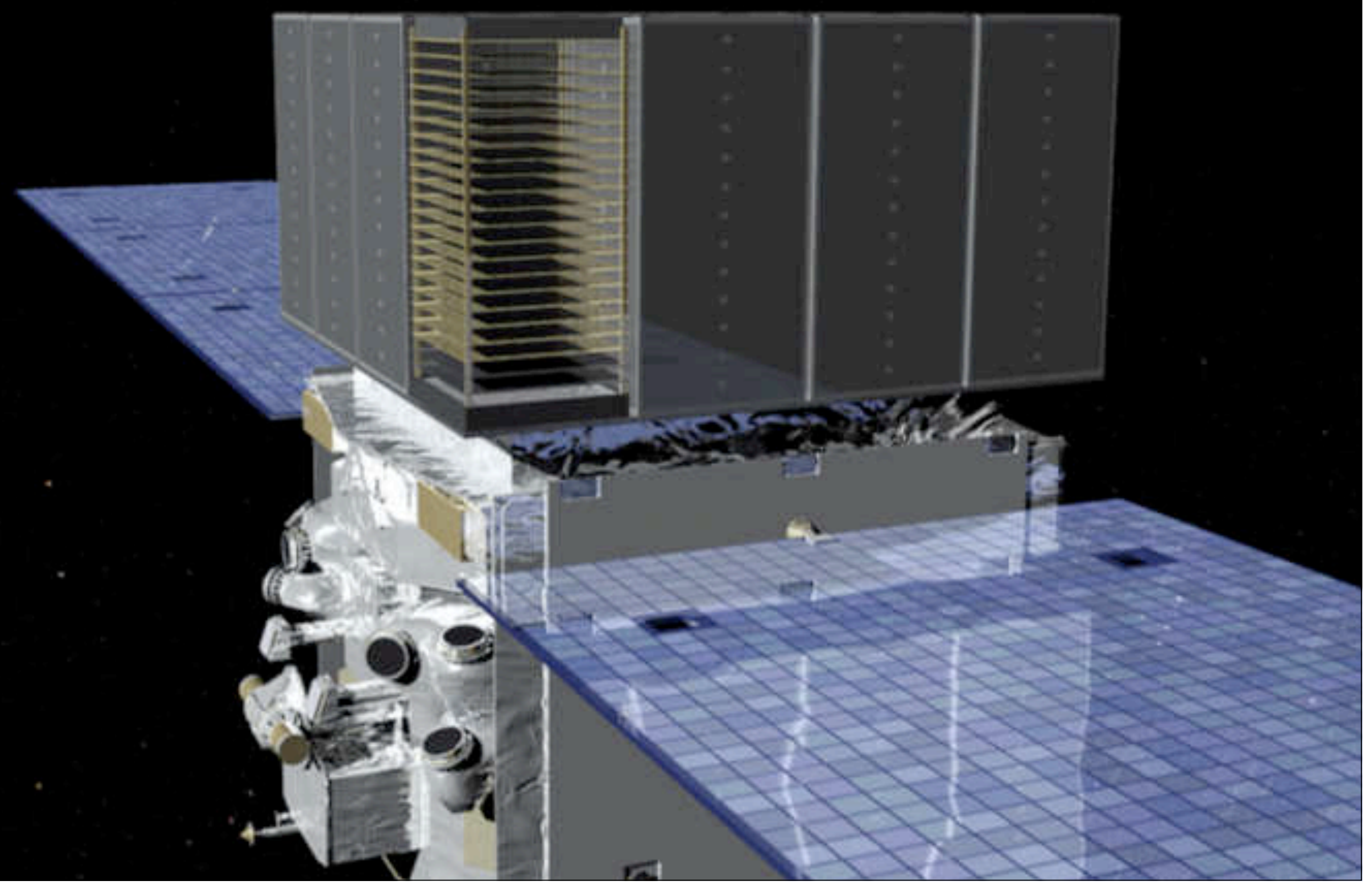




\section{GRB 0809 / 6C \\ Spectrum : up to $\sim 10 \mathrm{GeV}$ (obs.)}

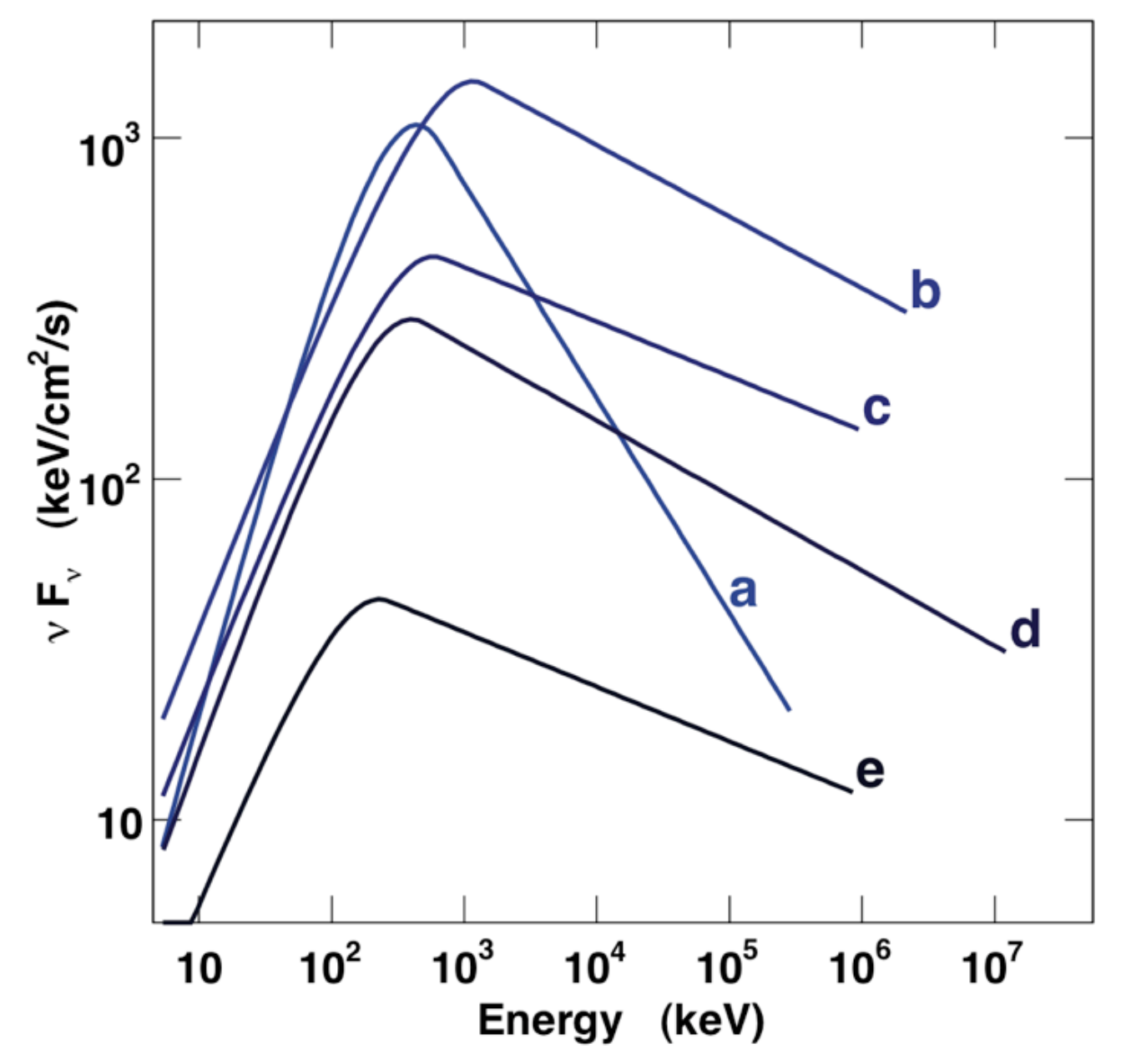

- "Band" (broken power-law) fits, joint GBM/LAT, in all time intervals

- "Soft-to-hard" spectral time evolution

- Long-lived $\left(10^{3} \mathrm{~s}\right)$ $\mathrm{GeV}$ afterglow

- Little evidence for 2nd spectr. comp. (in some cases) 


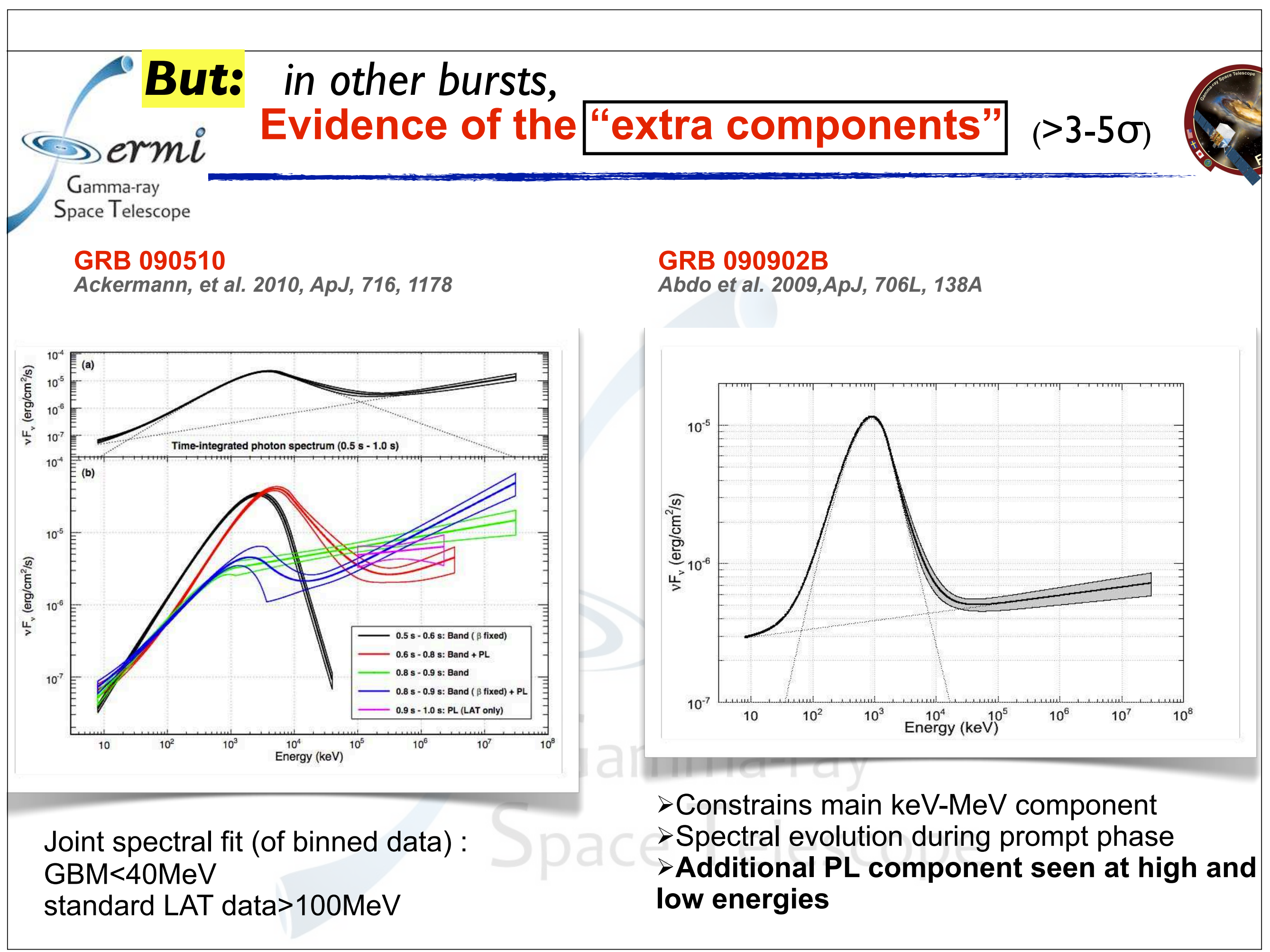




\section{Some observed photon energies and redshifts}

\begin{tabular}{|c|c|}
\hline$E_{\text {obs }}(\mathrm{GeV})$ & $z$ \\
\hline 13.2 & 4.35 \\
\hline 7.5 & 3.57 \\
\hline 5.3 & 0.74 \\
\hline 31.3 & 0.90 \\
\hline 33.4 & 1.82 \\
\hline 19.6 & 2.10 \\
\hline 2.8 & 0.897 \\
\hline 4.3 & 1.37 \\
\hline
\end{tabular}

- Even $z>4$ bursts result in $E_{\text {obs }} \sim 10 \mathrm{GeV}$ photons - Some z l bursts produce $E_{\text {obs }} \geq 30 \mathrm{GeV}$ photons ( I $30 \mathrm{GeV}$ in rest frame!)

- $\Rightarrow$ encouraging

for low $E_{\text {th }} A C T s:$ HAWC, CTA... 


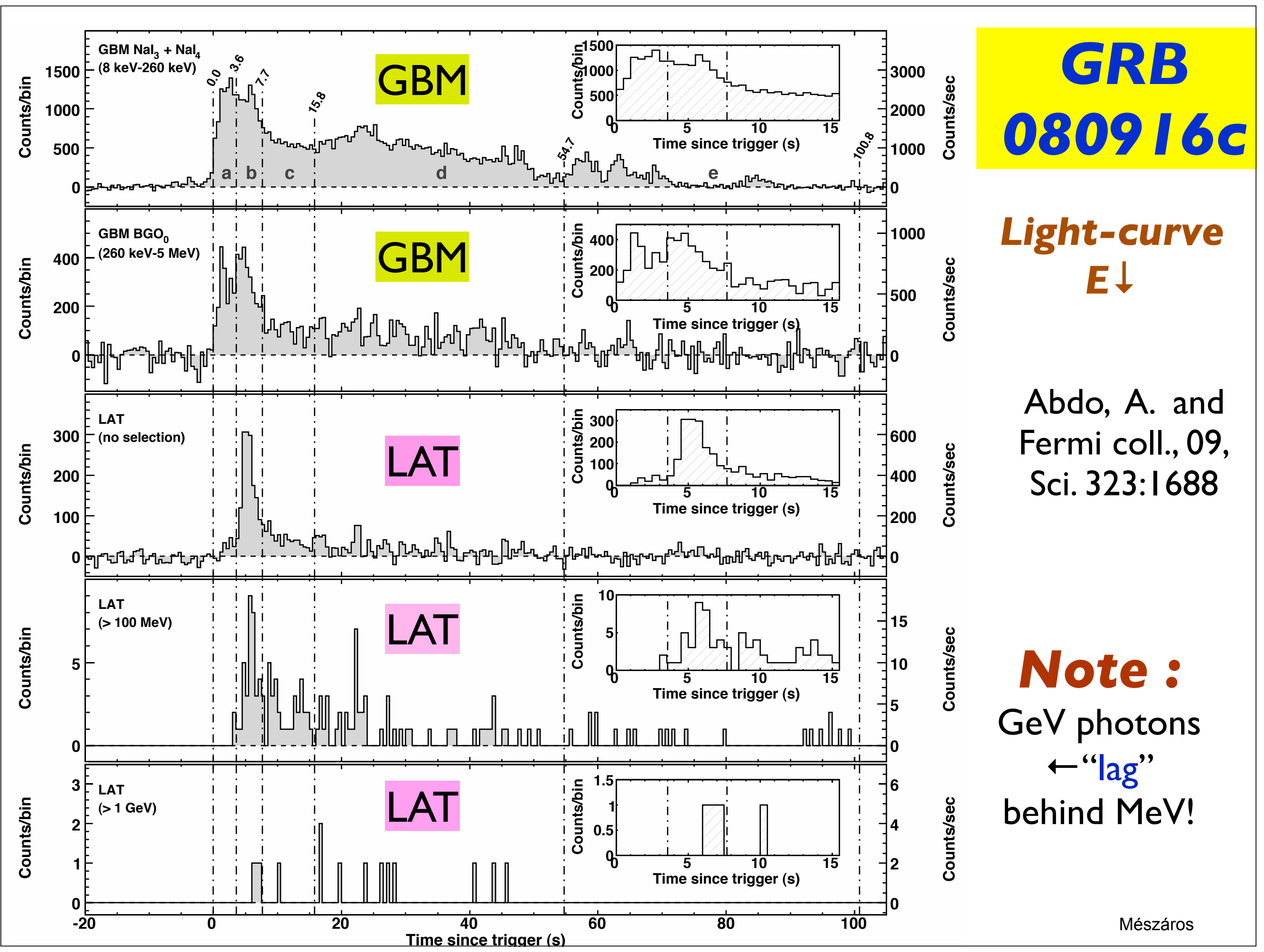




\section{(A) Evolving Fireball paradigm:}

Old paradigm: internal + external shock

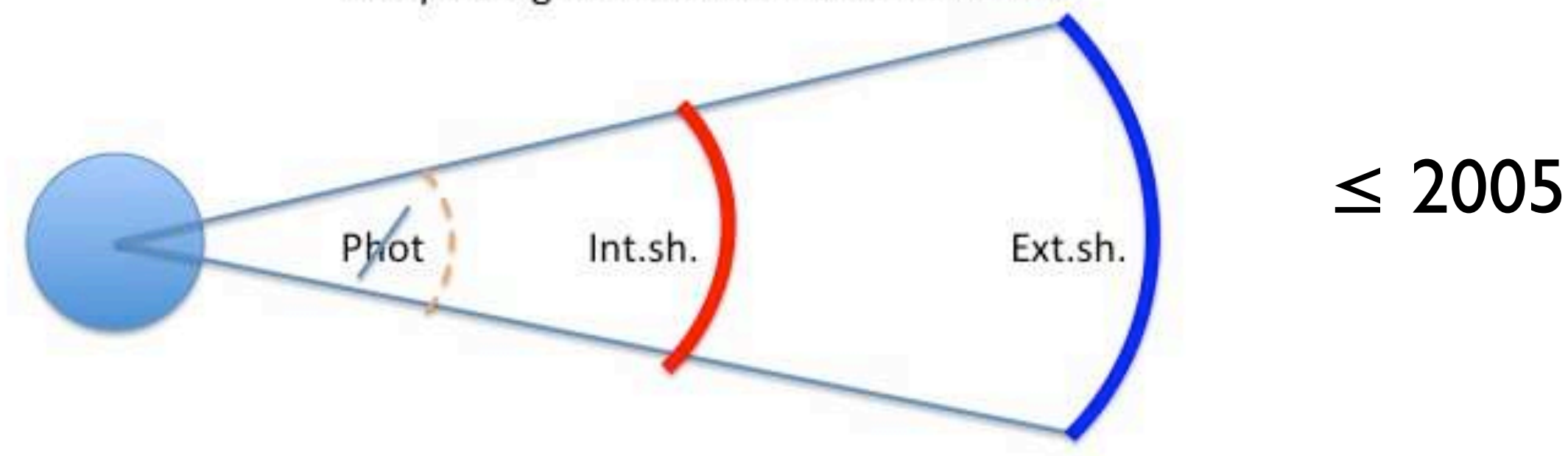

New paradigm: photosphere + external shock

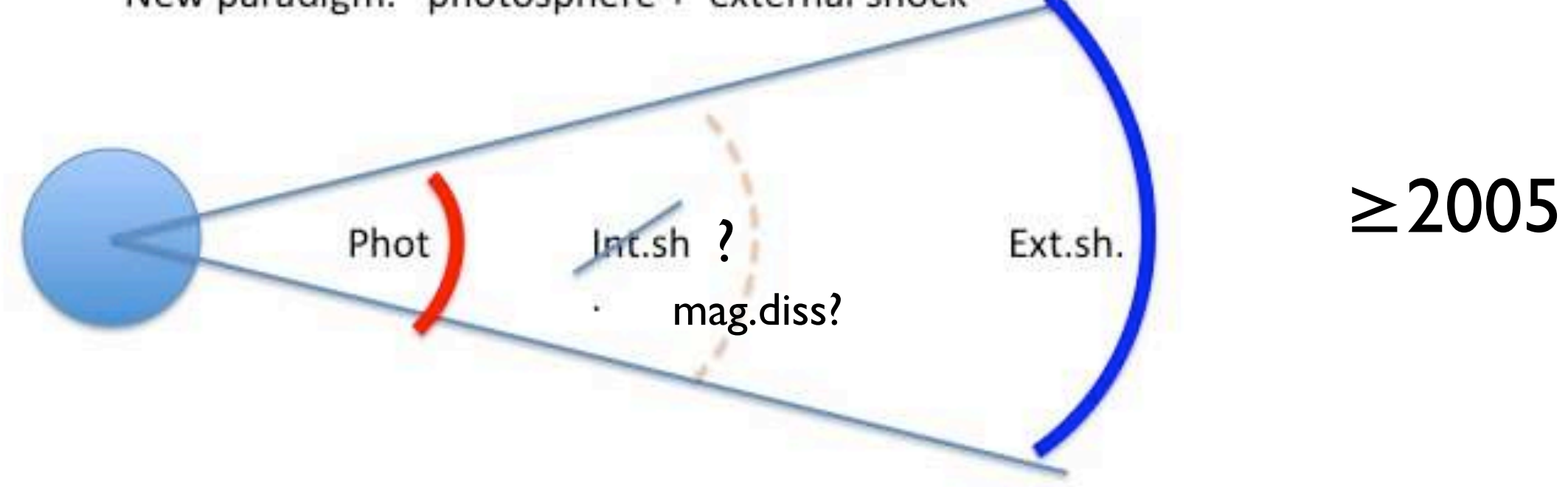




\section{Recent thrusts in exploring the prompt emission:}

A) De-emphasize internal shocks (inefficent)

$\rightarrow$ dissipative photospheric models

or:

B) Modify internal shocks : slow heating,

(i) turbulence behind shocks (Fermi 2nd ord),

(ii) magnetic dissipation (high rad. efficiency),

(iii) hadronic cascades (naturally slower heat'g) 


\section{A "leptonic" model:}

Toma,Wu, Mészáros, 20II, MN 4I5: 1663

\section{Photosphere and internal shock of the GRB jet}

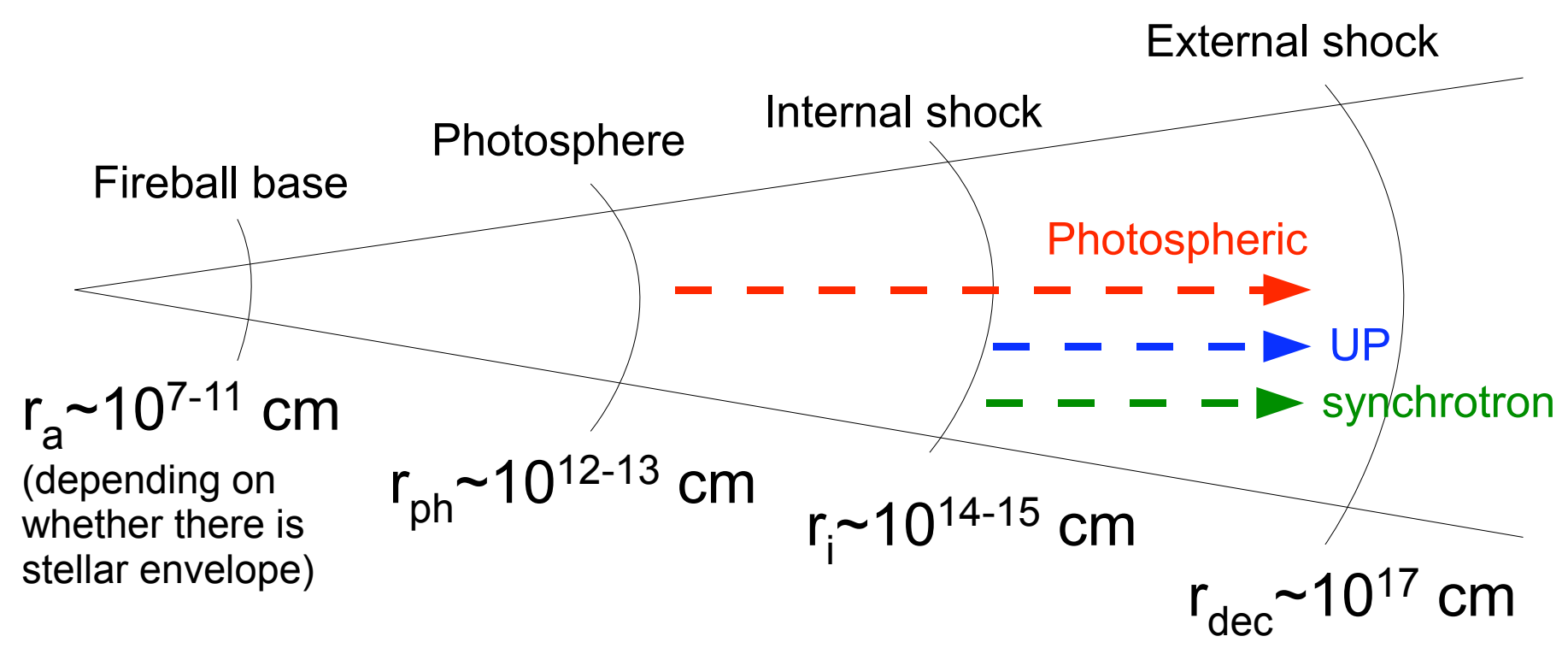

The photospheric emission can naturally provide a high $\gamma$-ray efficiency and the typical photon energy of the Band spectrum, $\sim 1 \mathrm{MeV}$ (Paczynski 86; Goodman 86).

The dissipation below the photosphere could cause the emission to be non-thermal (Meszaros \& Rees 00; Rees \& Meszaros 05; Pe'er et al. 05; Ioka et al. 07; Beloborodov 09) 


\section{p-n coll. $\rightarrow$ e $\pm \rightarrow \gamma$-spectrum}

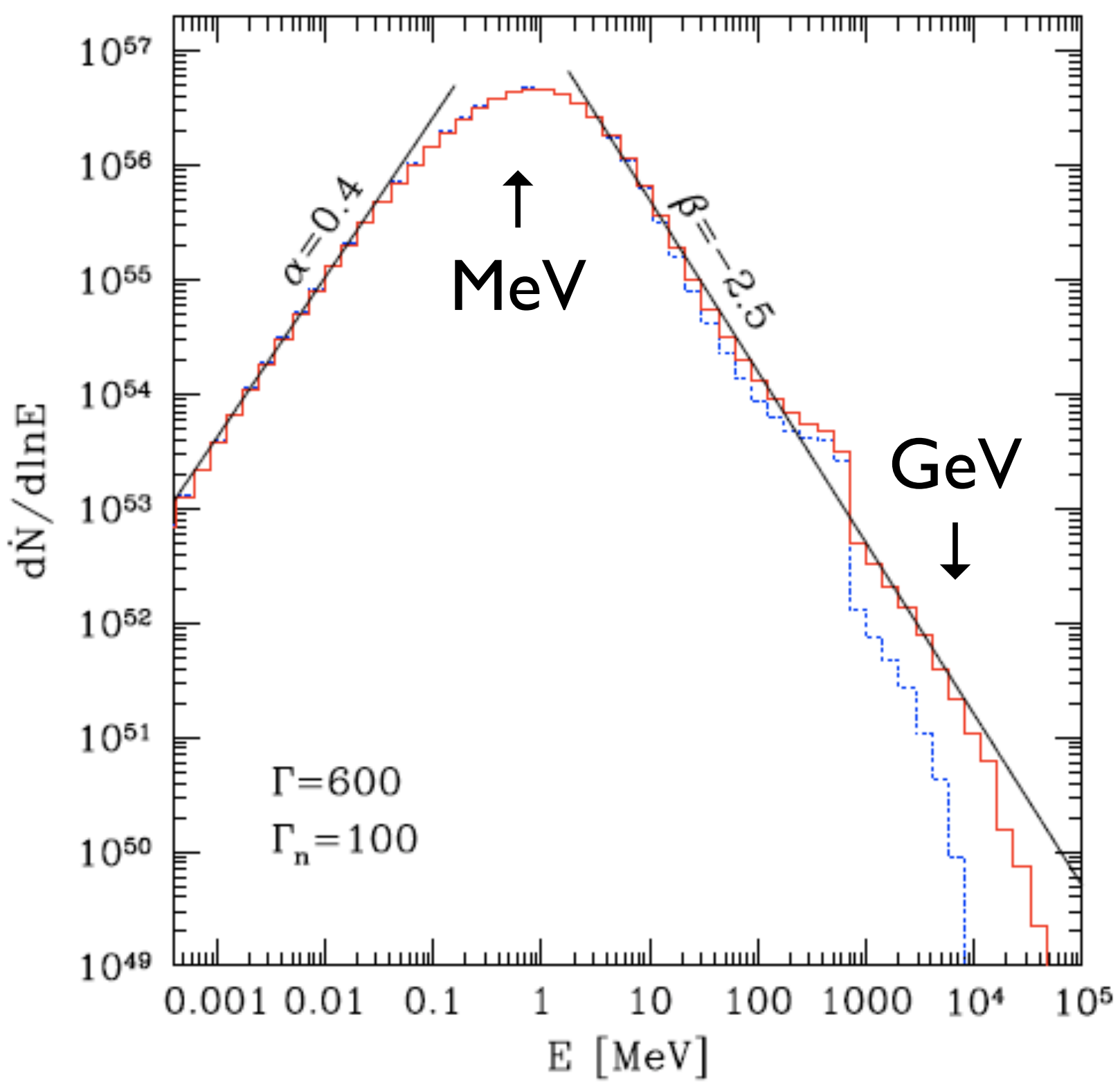

- The result is a thermal peak at the $\sim \mathrm{MeV}$ Band peak, plus

- a high energy tail due to the non-thermal $\mathrm{e}^{ \pm}$, whose slope is comparable to that of the observed Fermi bursts with a "single Band" spectrum

- The "second" higher energy component (when observed) must be explained with something else

(Beloborodov, 20I0) 


\section{Self-consistent hadronic int. shock}

Calculate self-consistent CR proton, photon \& neutrino spectra

- Originally: Waxman \& Bahcall' 97 consider standard int. shock as leptonic for photons,
hadronic for $\mathrm{p}, \gamma \rightarrow v$ leptonic for photons,
hadronic for $\mathrm{p}, \gamma \rightarrow V$ $\measuredangle$ Afterglow

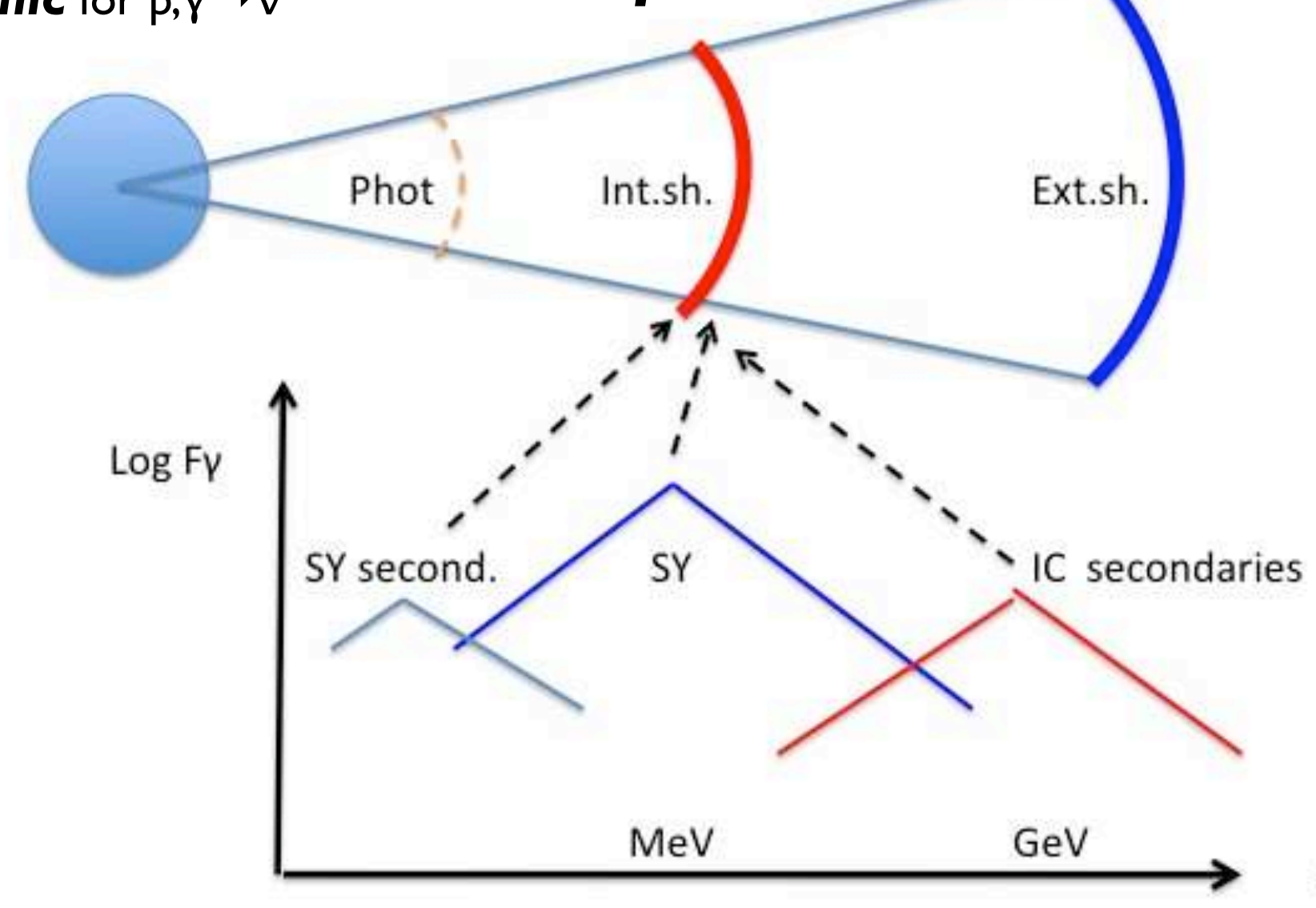

FS: X-ray, etc.; RS: Opt. flash

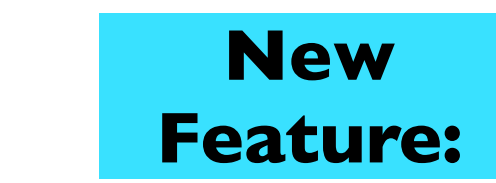

\section{Feature:}

Hadron accel. + photomeson $\rightarrow$ "dissipation" $\rightarrow$ inject copious relativistic sec'y leptons

- $\measuredangle$ Asano \& PM,

09-12 on, calculate second'y photons \& second'y neutrinos from both original \& hadronic sec'y leptons 


\section{IS w. hadronic cascades, $\gamma$}

Murase, Asano, Terasawa \& PM' I 2,ApJ746:I64

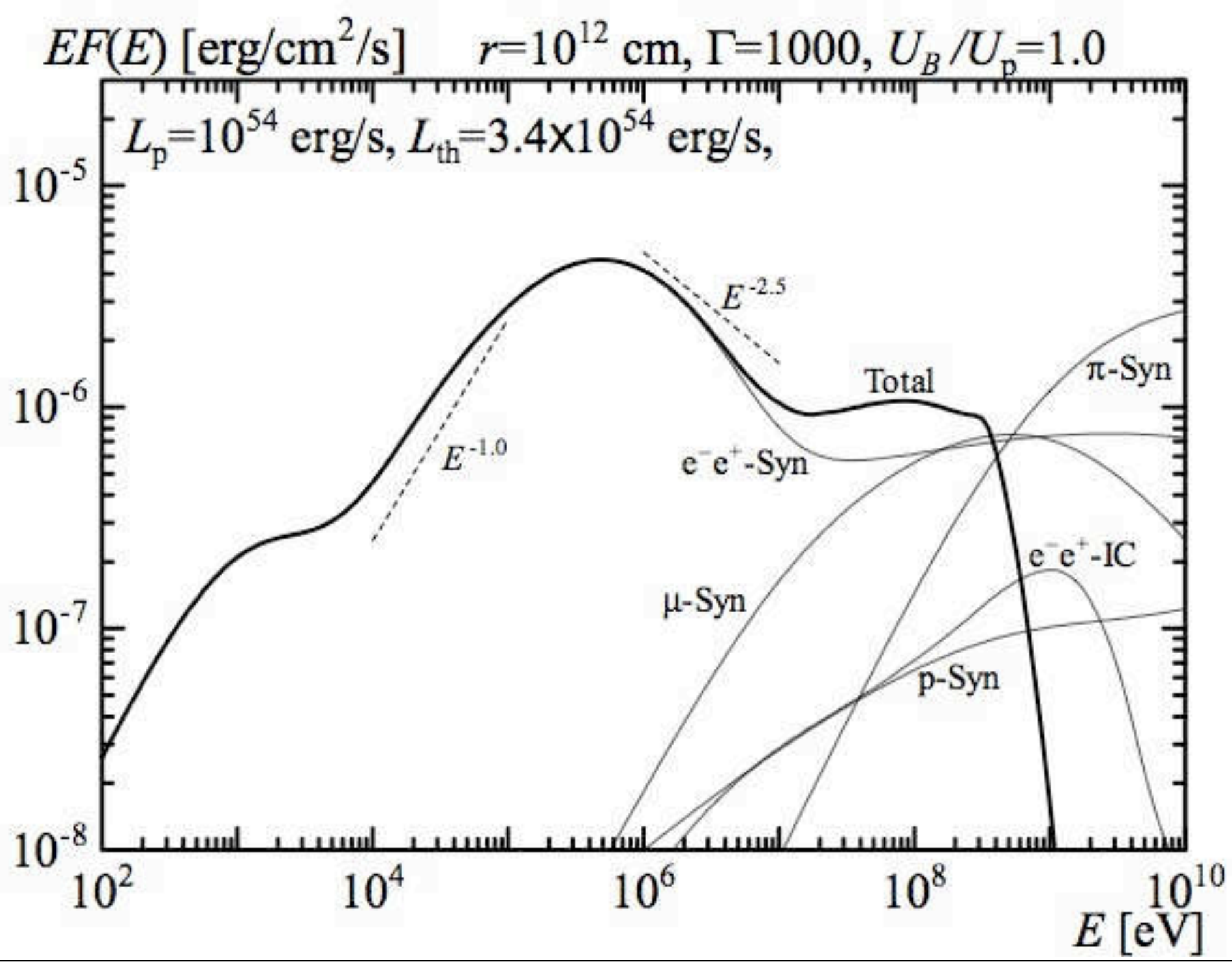




\section{Universal Diffuse Number Flux of Photons}

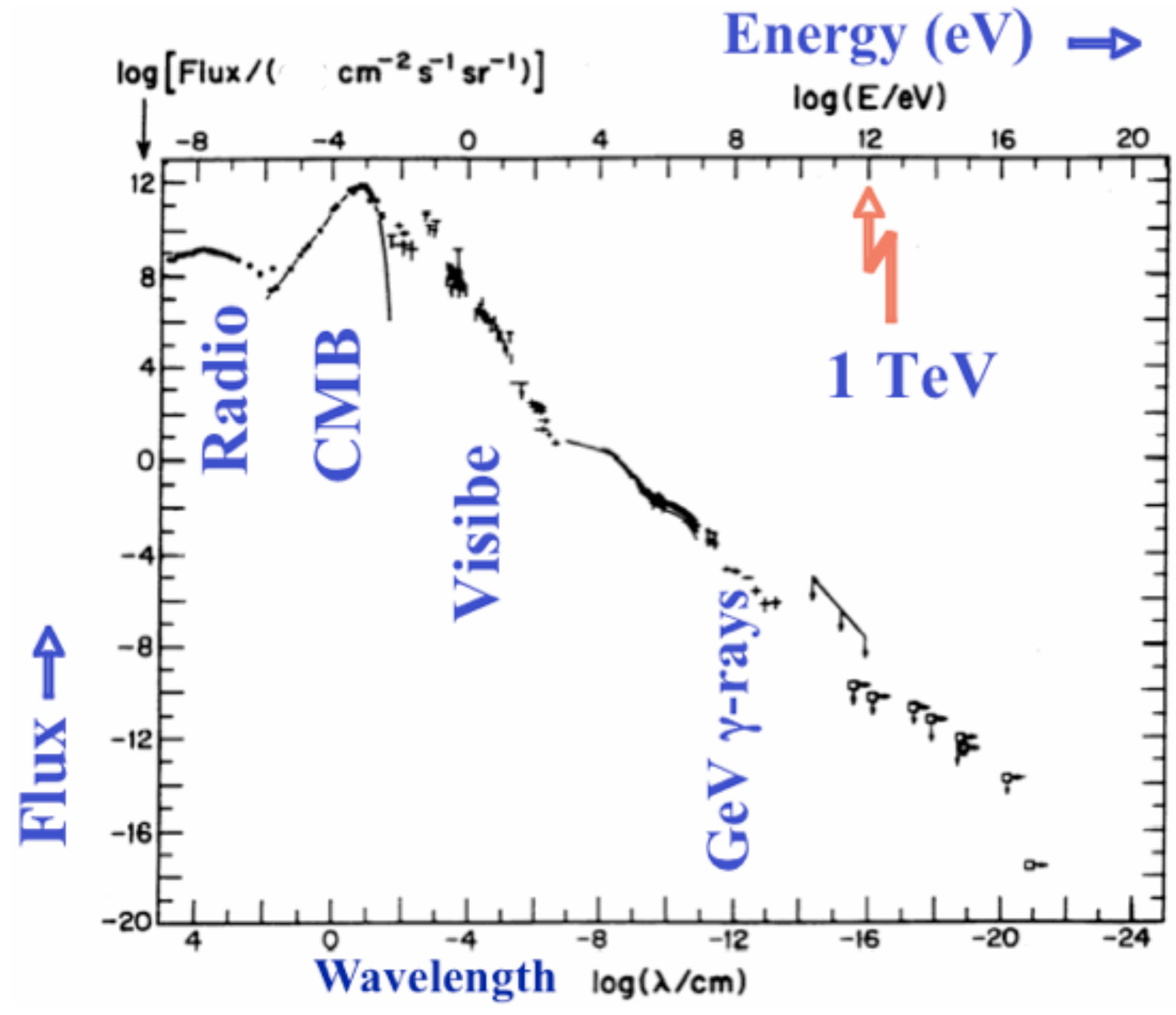




\section{Aggregate of all sources: \\ $\rightarrow$ diffuse radiation background}

IXB = isotropic $(X)$ background where

IGB, INB, ICRB is

Isotropic $\mathrm{Y}, \mathrm{V}, \mathrm{cr} \mathrm{bkg}$. 


\section{Universal Diffuse Number Flux of Photons \& Cosmic Rays}

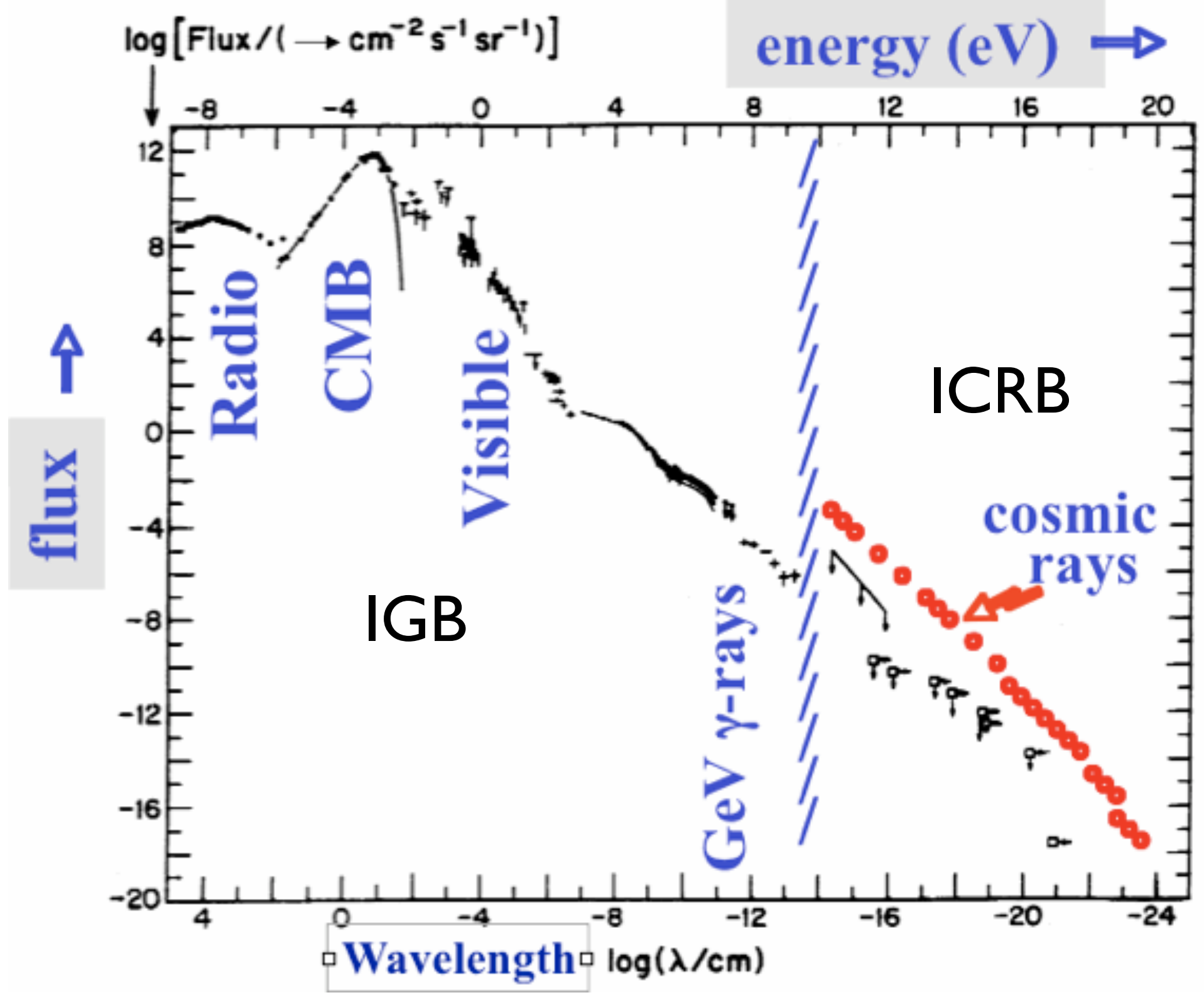




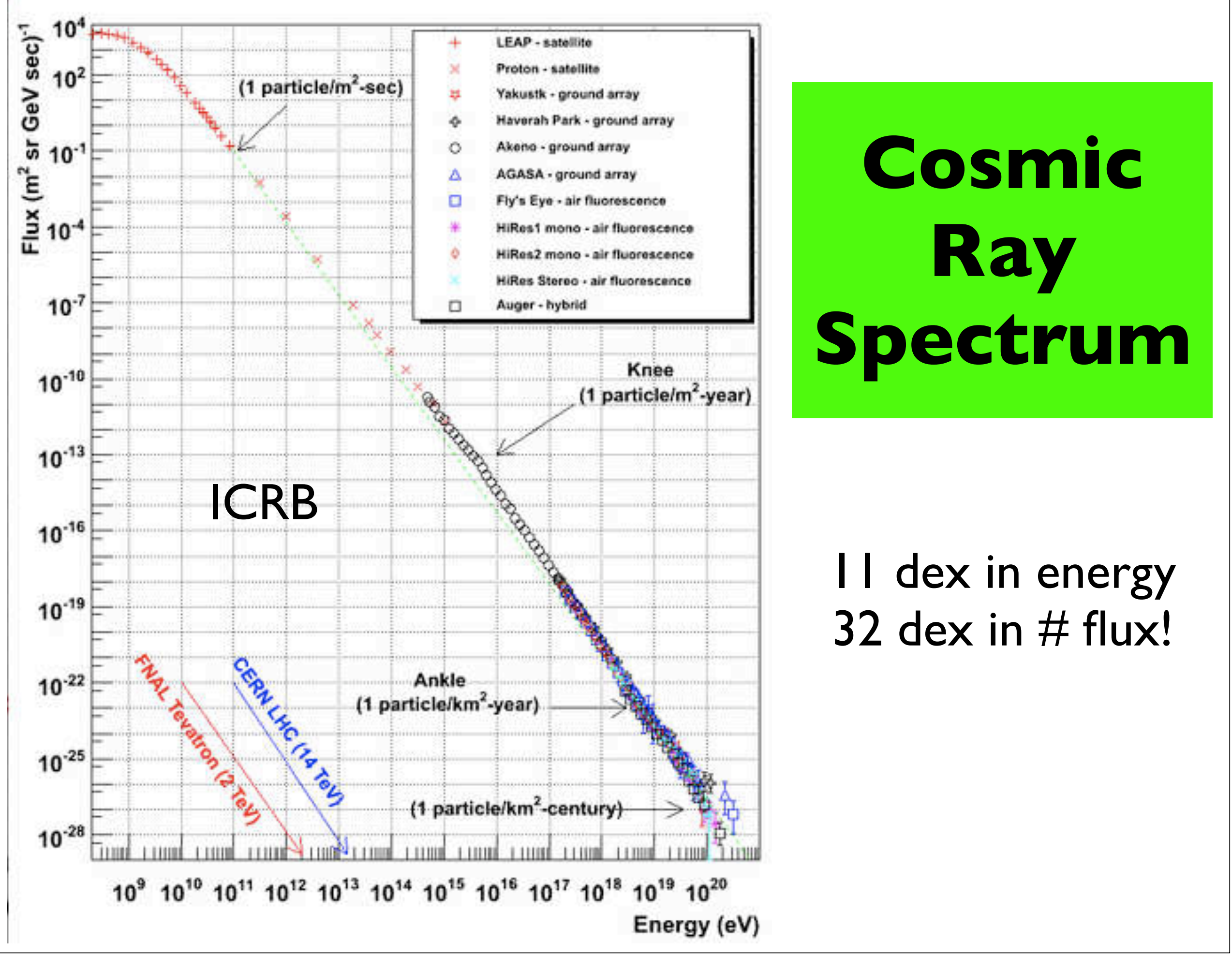




\section{Cosmic ray flux and Composition}

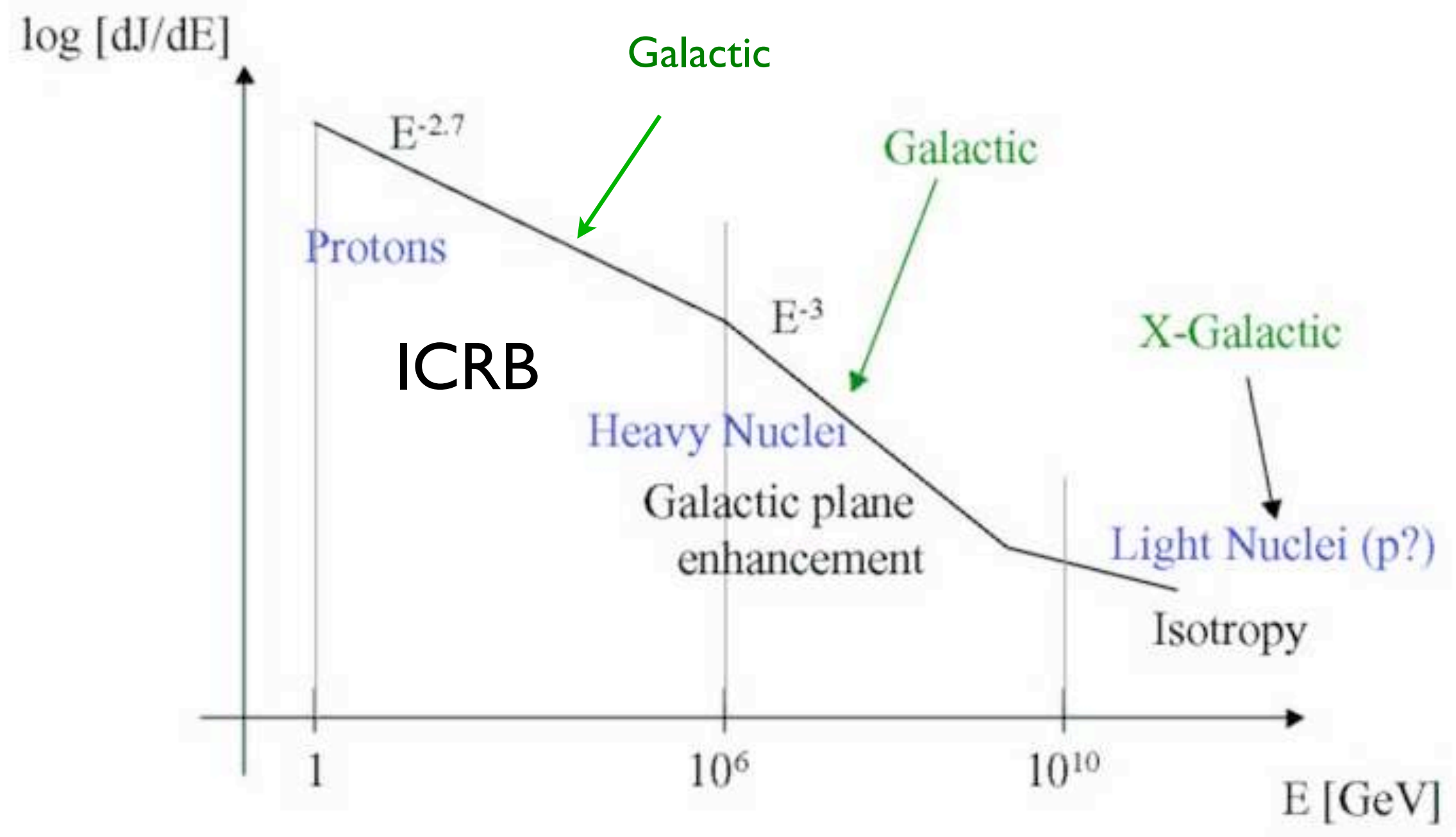

$U_{c r}(1 \mathrm{GeV})=1 \mathrm{eV} / \mathrm{cm}^{3}$ 


\section{Universal Diffuse Number Flux of Neutrinos}

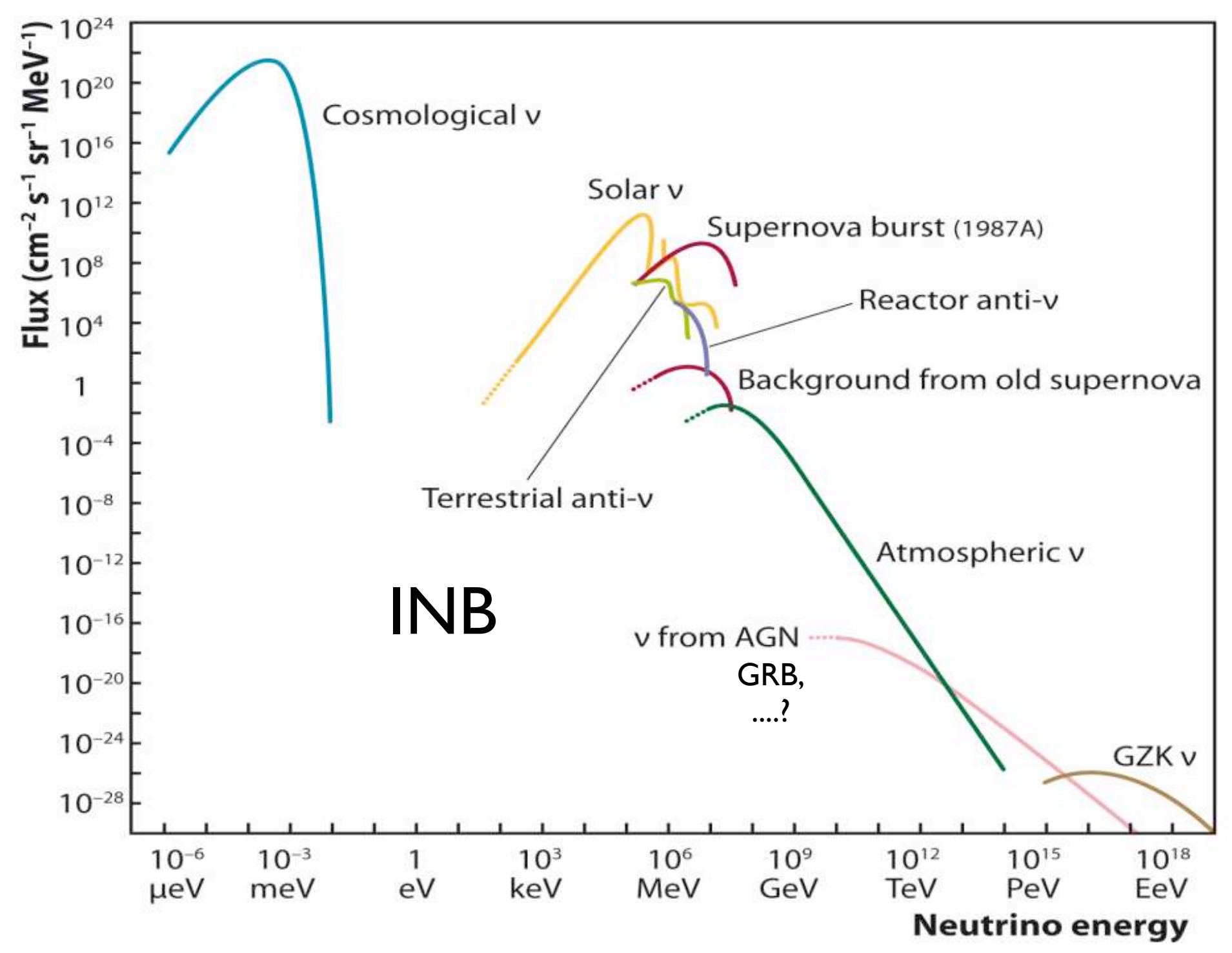




\section{Electromagnetic vs. Neutrino Energy Spectral Domains}

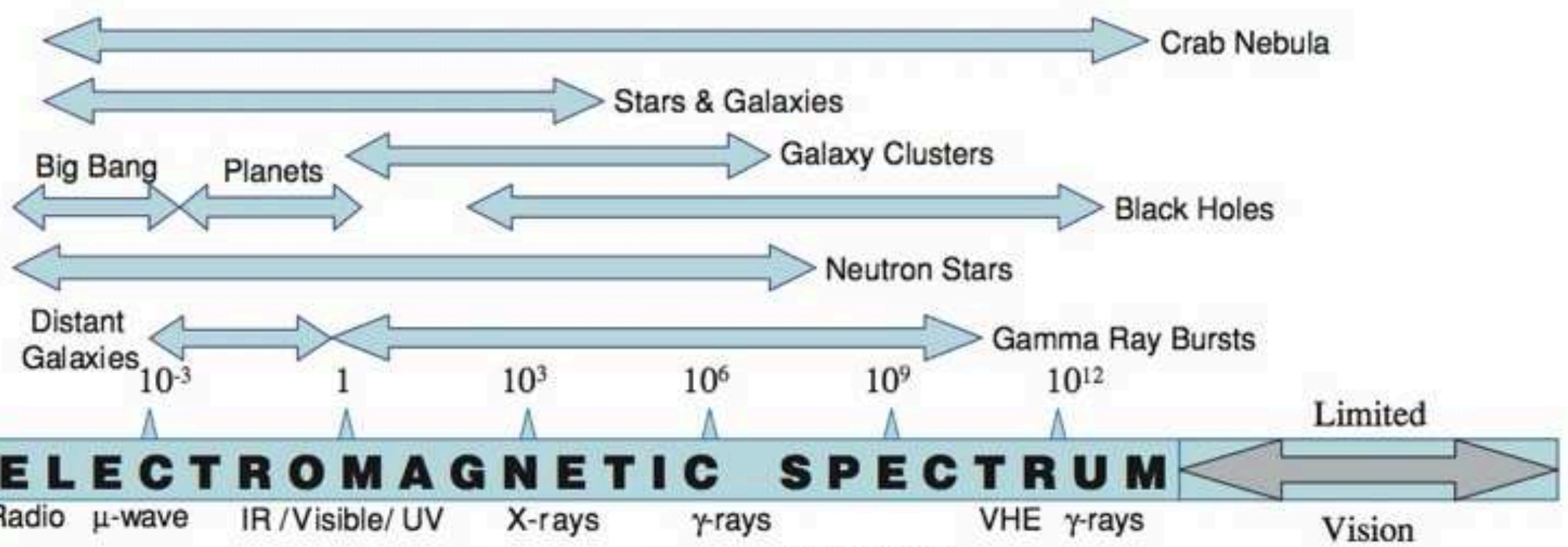

Radio $\mu$-wave

IR / Visible/ UV

X-rays $\quad$-rays

Accelerators

Reactors,

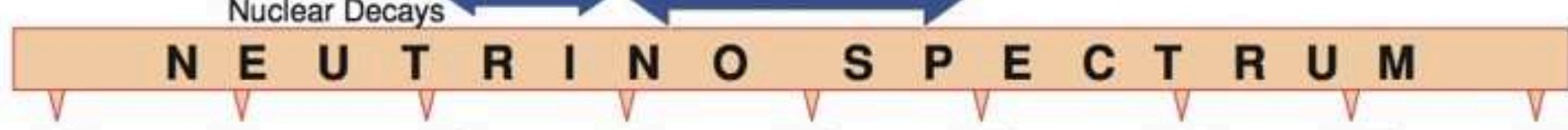

$10^{-3}$

1

$10^{3}$

$10^{6}$

$10^{9}$

$10^{12}$

$10^{15}$

$10^{18}$

$10^{21} \mathrm{eV}$

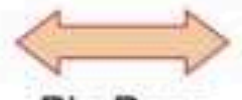

Sun $<$

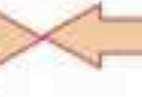

$$
\text { Stars }
$$

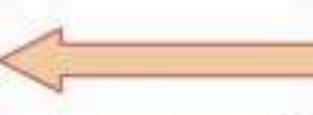

Crab Nebula

Atmospheric Neutrinos
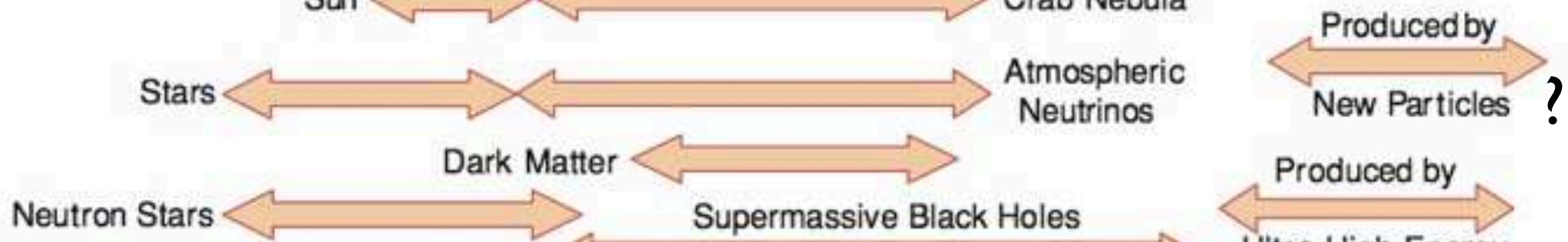

Dark Matter

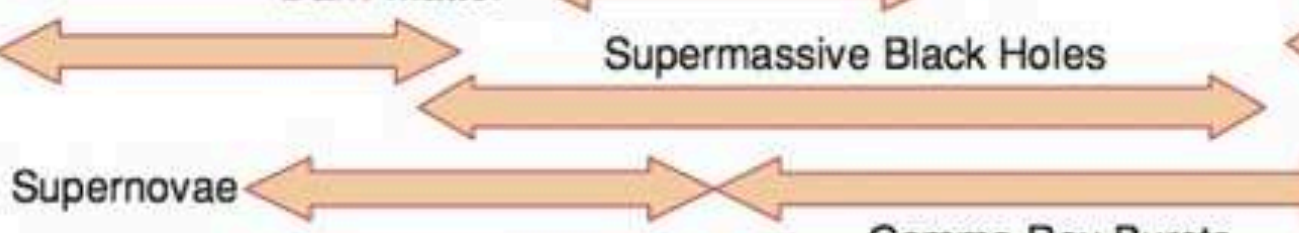
Produced by

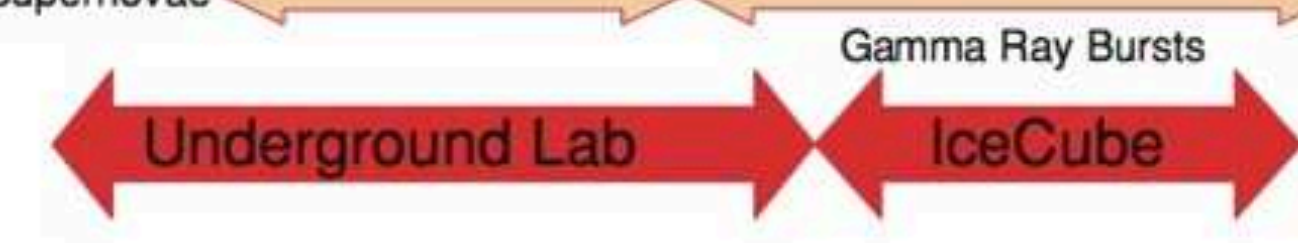




\section{What is the Relation} between

\section{UHECRs and UHENUs?}

UHECR = Ultra-High Energy Cosmic Rays UHENU $=$ Ultra-High Energy Neutrinos

$$
\text { define } \quad \begin{aligned}
\mathrm{HE} & \approx 10^{9} \mathrm{eV}(\mathrm{GeV}) \\
\mathrm{VHE} & \approx 10^{12} \mathrm{eV}(\mathrm{TeV}) \\
\mathrm{UHE} & \approx 10^{18} \mathrm{eV}(\mathrm{EeV})
\end{aligned}
$$




\section{Cosmic Rays and Neutrinos}

Driving theme: Origin of Cosmic Rays

(Sullivan 14)

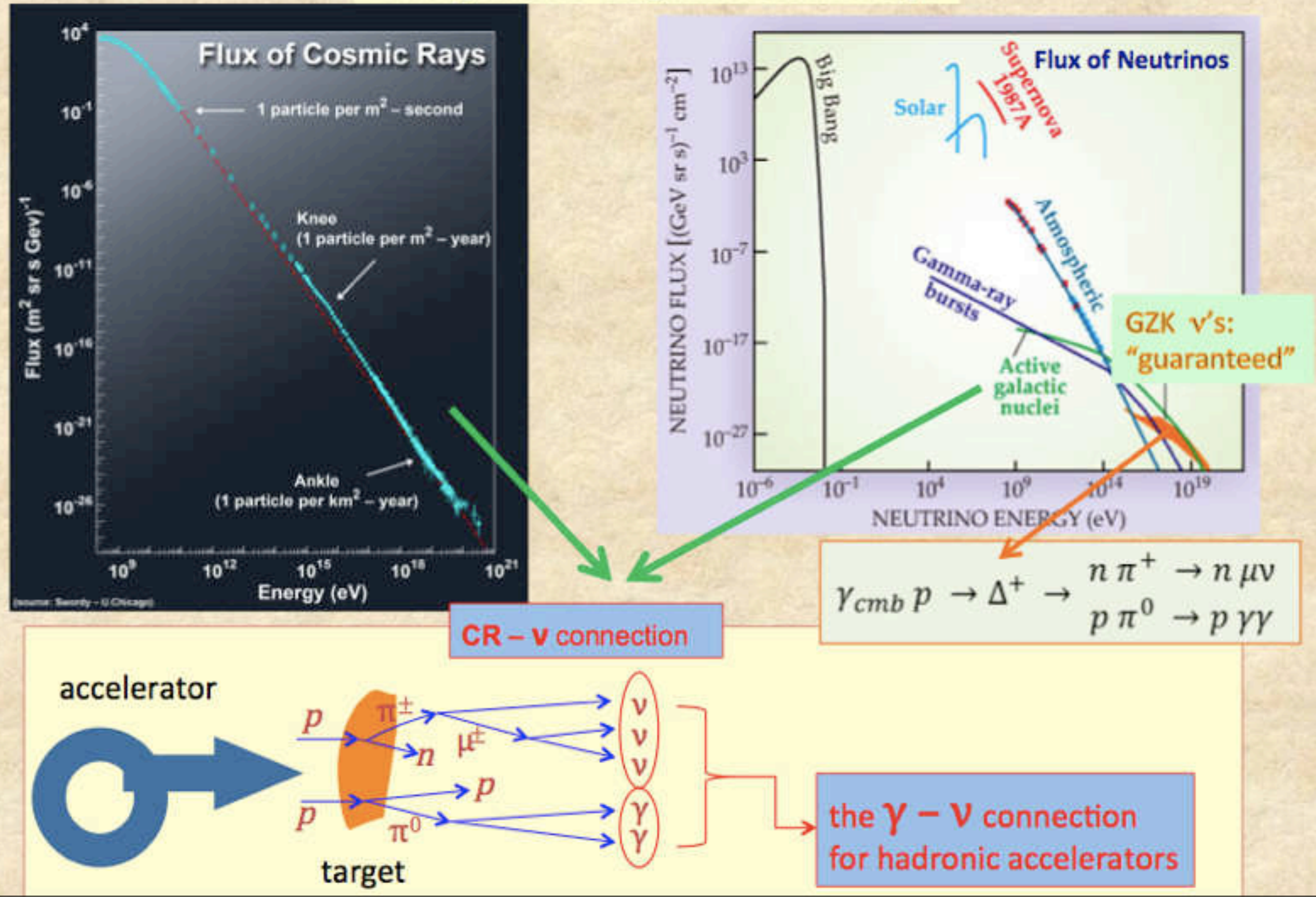




\section{Why Neutrinos?}

Neutrinos are ideal astrophysical messengers

- Travel in straight lines

- Very difficult to absorb in flight

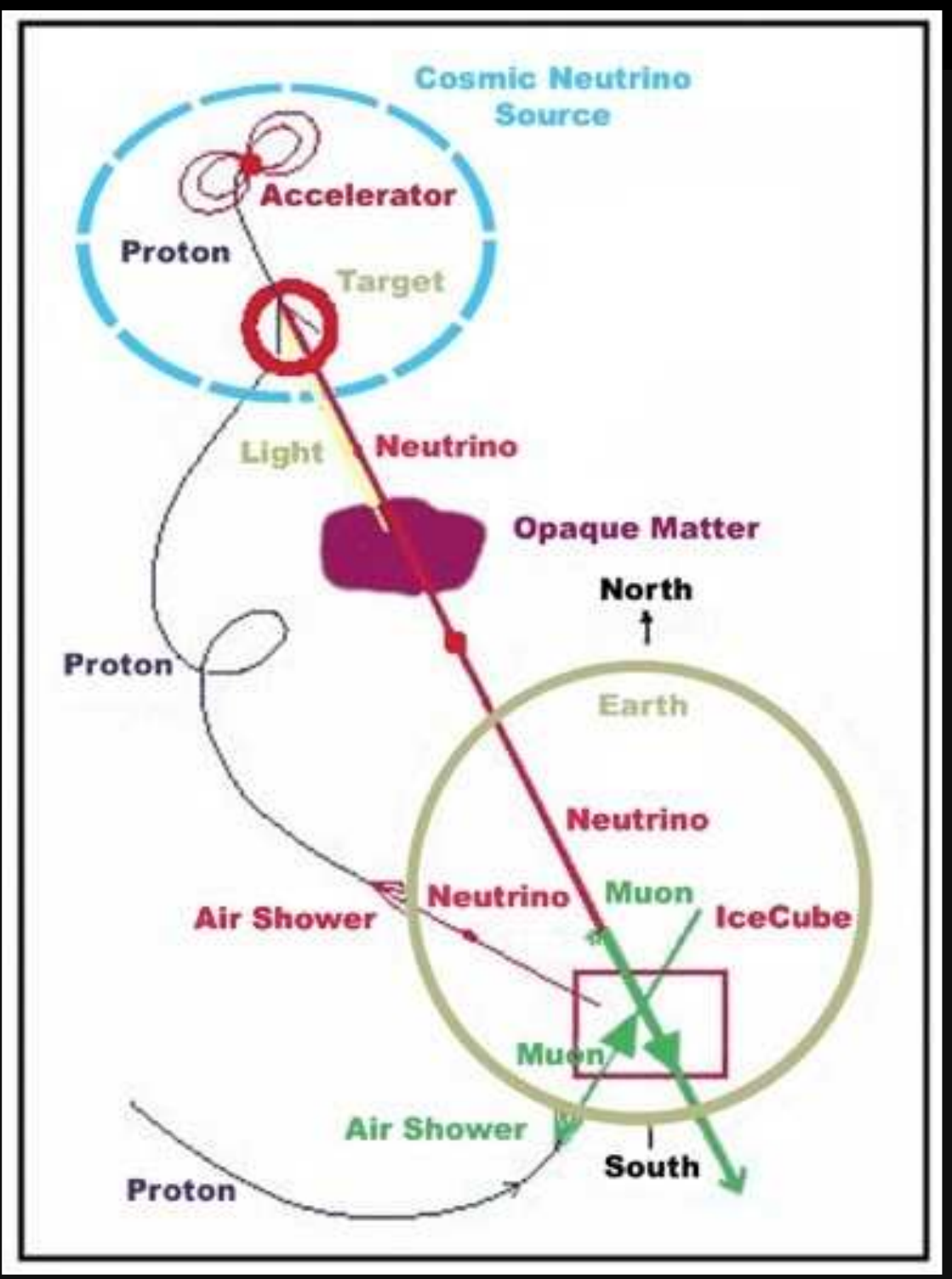




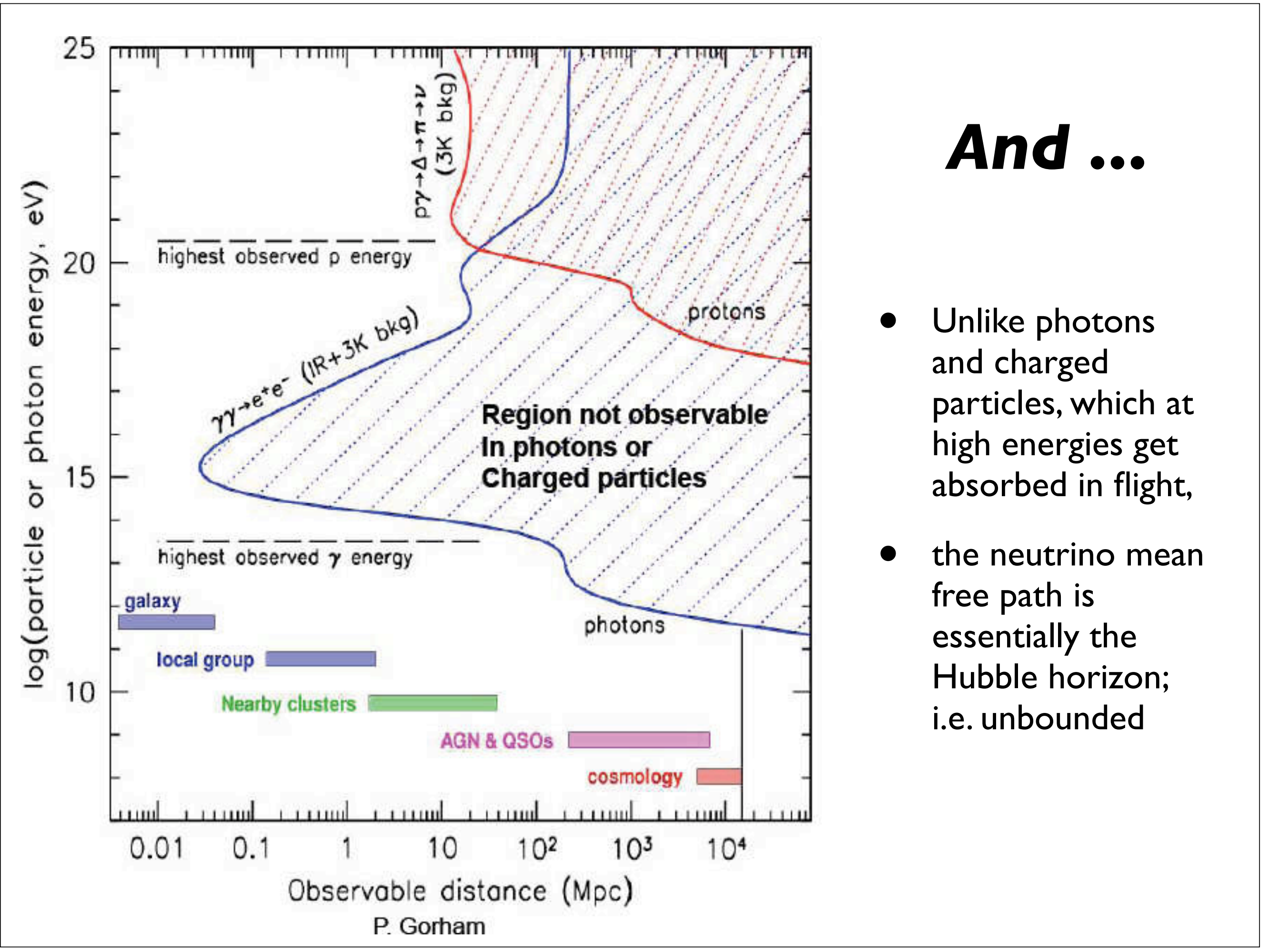




\section{UHECR : maximum energy?}

gyroradius: $\mathrm{r}_{\mathrm{L}} \sim \mathrm{ct}_{\mathrm{gy}} \sim \mathrm{m}_{\mathrm{p}} \mathrm{c}^{2} \gamma / \mathrm{ZeB}=\varepsilon_{\mathrm{p}} / \mathrm{ZeB}<\mathrm{R}$ (size of accel.)

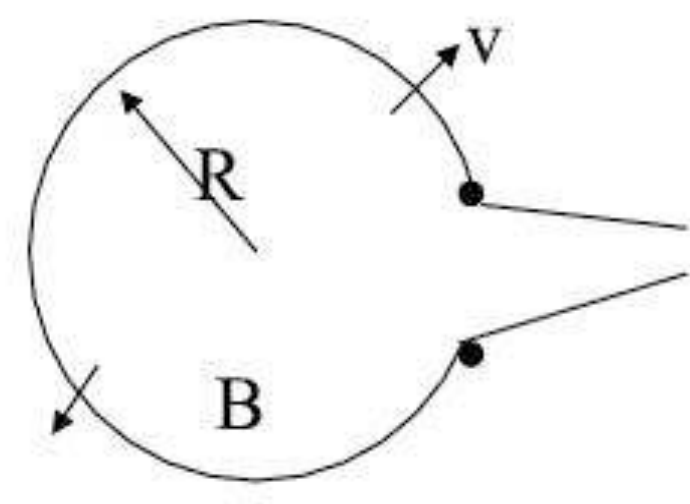

or (EM analog):

$$
V=\frac{1}{c} \dot{\Phi} \sim \frac{1}{c} \frac{B R^{2}}{R / \mathrm{v}}=\beta B R
$$$$
\longrightarrow \varepsilon_{p}<\beta e B R
$$

$$
\Rightarrow L>4 \pi R^{2} \frac{B^{2}}{8 \pi} v>\frac{1}{2 \beta}\left(\frac{\varepsilon_{p}}{e}\right)^{2} c
$$

But if relativistic expansion, bulk Lorentz factor $\Gamma>>1$, then time ${ }_{\text {obs }} \sim \mathrm{R} / \mathrm{c} \Gamma$, and size $\mathrm{obs}_{\mathrm{o}} \sim \mathrm{R} / \Gamma$, hence need

$$
\Rightarrow L>2 \frac{\Gamma^{2}}{\beta} \varepsilon_{p, 20}^{2} \times 10^{45} \mathrm{erg} / \mathrm{s}
$$




\section{Maximum $E_{p}$ for various sources (Hillas plot)}

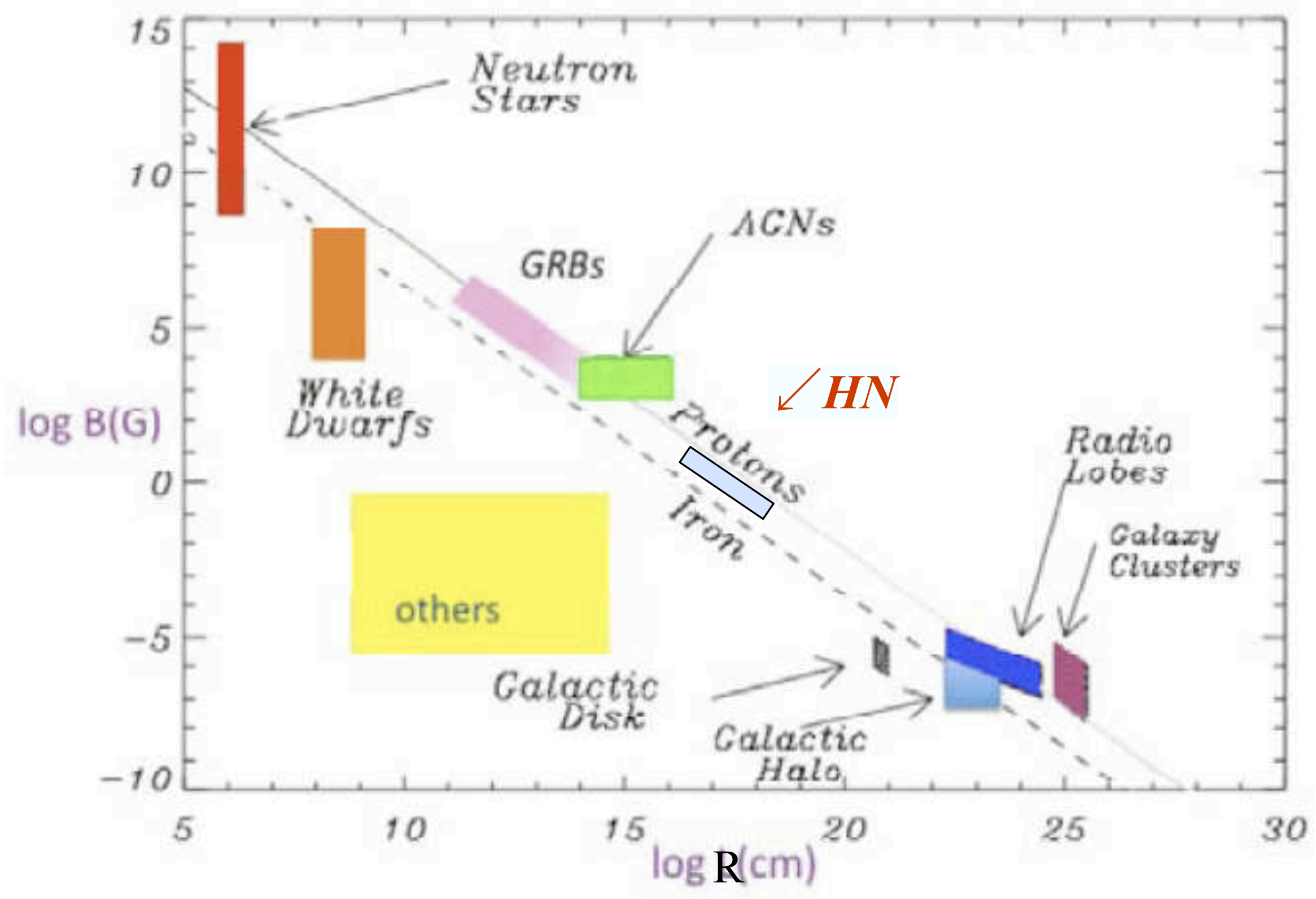




\section{GRB ? $E_{\max }:$}

- Require : $\left.r^{\prime}{ }_{L}=E^{\prime} / Z e B^{\prime} \geq R^{\prime}\right)$

- $\Rightarrow \quad E_{\max } \sim \Gamma Z$ e $B^{\prime} R^{\prime}$

- but, what are R', B' for a GRB?

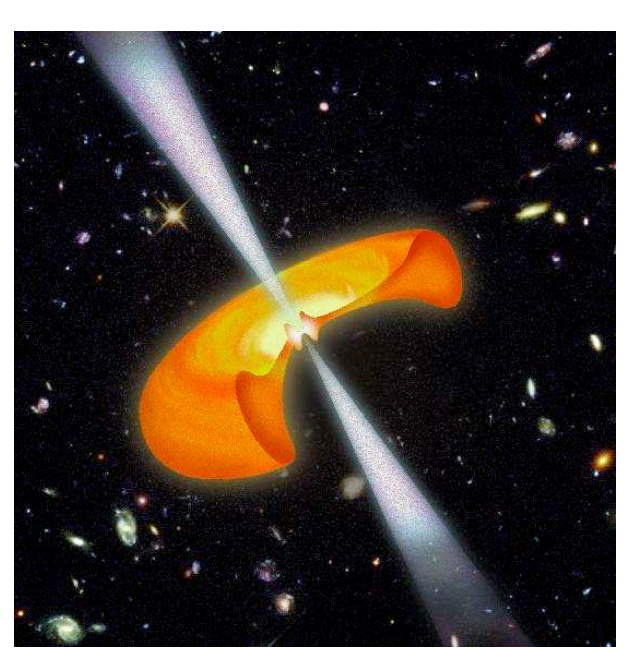

- primed: comoving;

- unprimed : lab frame;

- $\Gamma$ : jet Lorentz factor

- we have $\mathrm{R}^{\prime} \sim \mathrm{R} / \Gamma$; and external shock

occurs at $\mathrm{R}$ where $\mathrm{E}_{0} \sim \mathrm{n} \mathrm{m}_{\mathrm{p}} \mathrm{c}^{2} \mathrm{R}_{\operatorname{dec}^{3}} \Gamma^{2}$

$\rightarrow \mathrm{R} \sim \mathrm{R}_{\mathrm{dec}} \sim\left(\mathrm{E}_{0} / \mathrm{nm}_{\mathrm{p}} \mathrm{c}^{2}\right)^{1 / 3} \Gamma^{-2 / 3}$

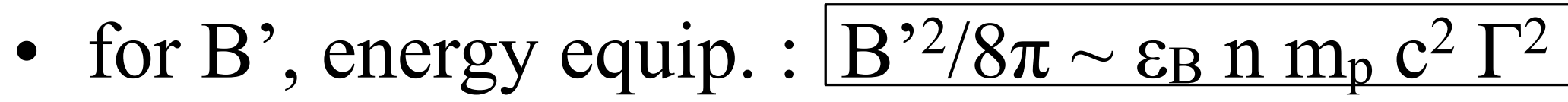

$\rightarrow \mathrm{B}^{\prime} \sim \varepsilon_{\mathrm{B}}{ }^{1 / 2}\left(8 \pi \mathrm{n} \mathrm{m}_{\mathrm{p}} \mathrm{c}^{2}\right)^{1 / 2} \Gamma$, so

- $\mathrm{E}_{\max } \sim \mathrm{Ze}\left(8 \pi \varepsilon_{\mathrm{B}}\right)^{1 / 2} \mathrm{E}_{0}{ }^{1 / 3}\left(\mathrm{n} \mathrm{m}_{\mathrm{p}} \mathrm{c}^{2}\right)^{1 / 6} \Gamma^{1 / 3}$, or

- $E_{\max } \sim 2 x 10^{20} Z E_{53^{1 / 3}} \varepsilon_{B,-2}{ }^{1 / 2} \Gamma_{2}^{1 / 3} n^{1 / 3} e V^{43}$ 


\section{AGN ? two main types ( $\sim 1 \%$ of all galaxies)}

Radio-loud: M87 (jet 10\%) (RL)

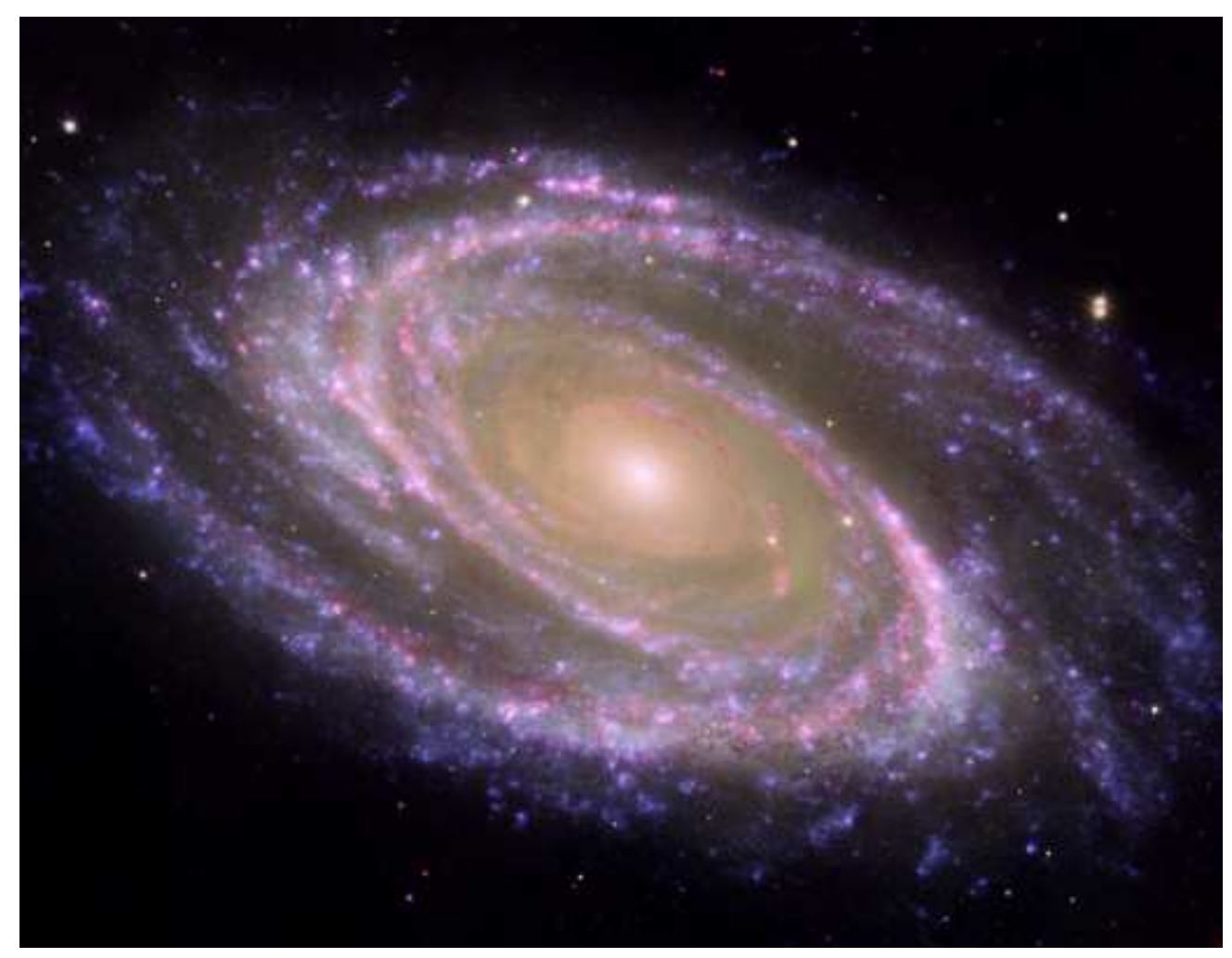

Radio-quiet: M81 (no jet $\underset{44}{\text { jet }}$ 90\%) (RQ) 


\section{RL AGN \\ as UHE $\gamma, C R$, v sources}

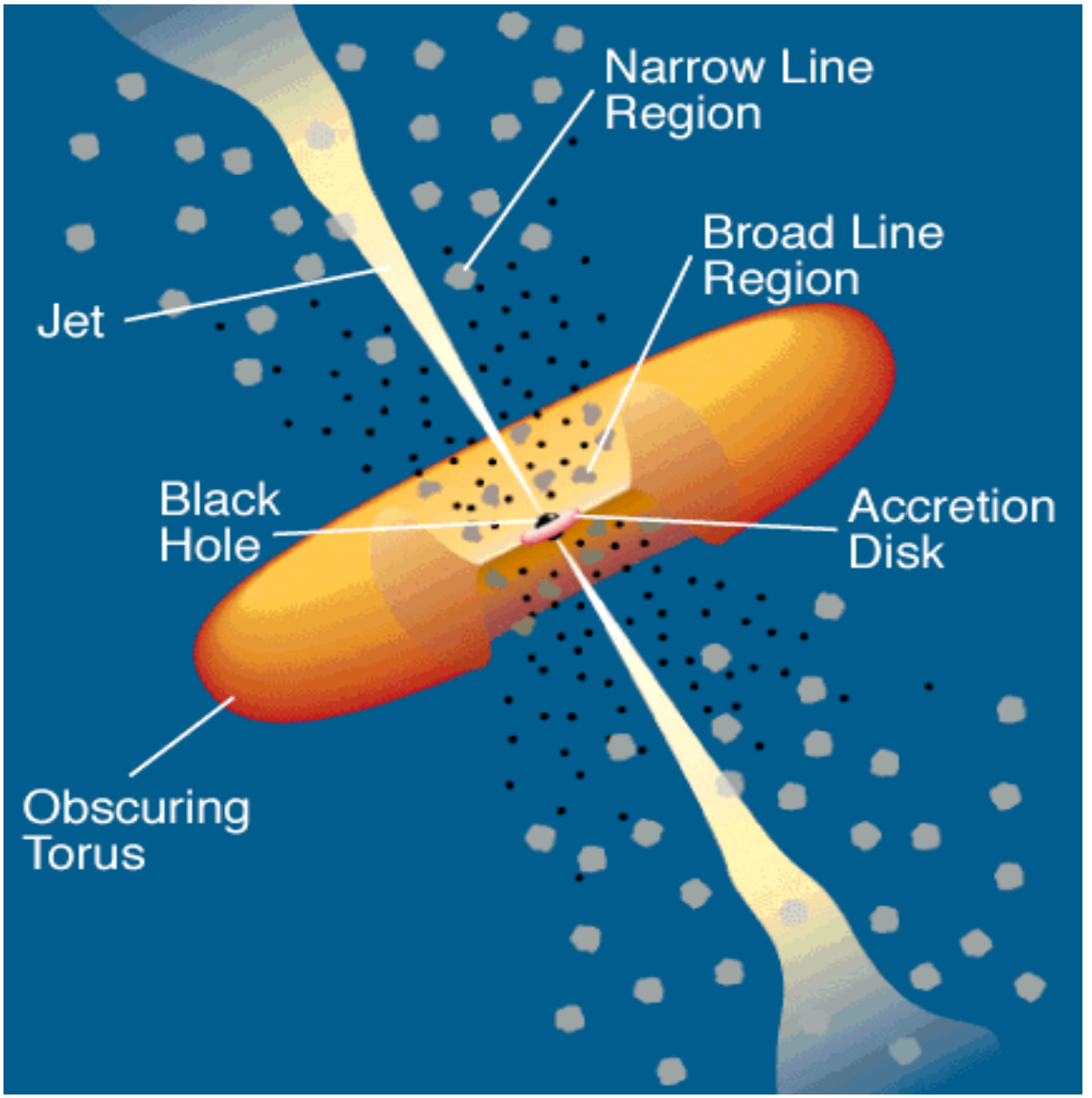

- Big brother of GRB: massive BH $\left(10^{7}-10^{8} \mathrm{M}_{\text {sun }}\right)$ fed by an accretion disk $\rightarrow$ jet

- But, jet $\Gamma_{\mathrm{j}, \mathrm{agn}} \sim 10-30$ ( while $\Gamma_{\mathrm{j}, \text { grb }} \sim 10^{2}-10^{3}$ )

- UV photons from disk; in addition, line clouds provide extra photons (+back-scatter)

- Typical ("leptonic") model: SSC (sync-self-compton); SEC(sync-exter.compton) 


\section{But: are RL (jet) AGNs the UHECR sources?}

- The AGNs in the VC catalog inside $75 \mathrm{Mpc}$ are generally weak, not strong-jet (radio-loud, RL) AGNs - and no longer statistically favored; but...

- There is possible evidence for :

a) large angle deflections (heavy elements); and b) non-jet (radio-quiet) AGNs are abundant...

- Independently, correlation with matter (normal galaxies) is strong 


\section{Alternative UHECR: RQ AGNs}

Pe’er, Murase, Mészáros, 2009, PRD 80, 123018 (arXiv:0911.1776)

- Could be that culprits are radio-quiet (RQ) AGNs

- Enough of them inside GZK radius

- Evidence for small jets in RQ AGNs

- Evidence for heavy CR composition (X $\mathrm{X}_{\max }$ Vs. E)

- Can accelerate heavy elements to right GZK energies, $E_{\max } \sim Z e B R \sim 10^{20} Z_{26} B_{-3} R_{10} e V \quad$ (if $\mathrm{B} \sim 10^{-3} \mathrm{G}, \mathrm{R} \sim 10 \mathrm{pc}$ )

- Can survive photo-dissociation

- Heavy elements have larger rms. deviation angles

- Correlation with matter (gal) distribution is good. 


\section{Another alternative: Hypernovae?}

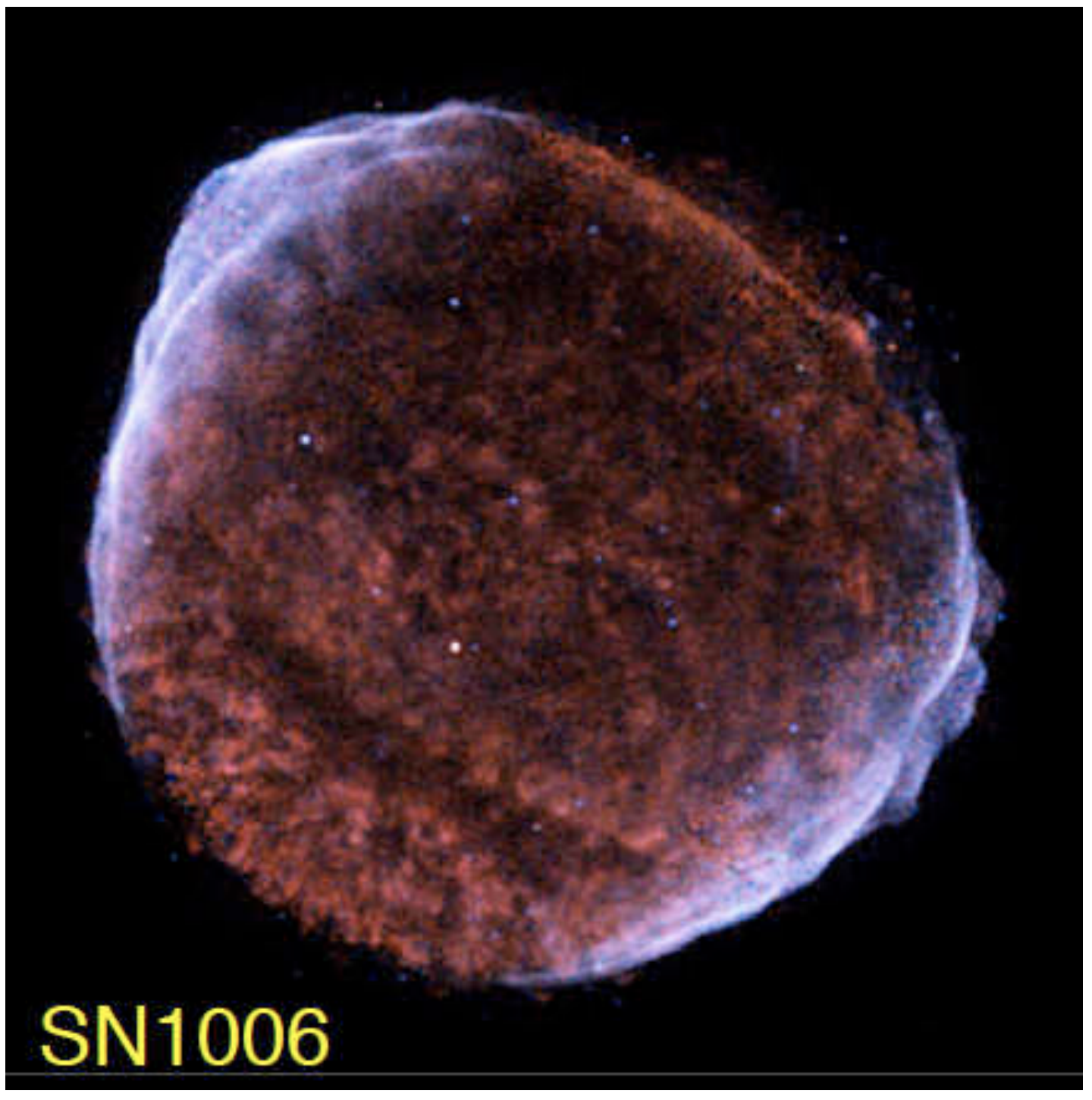

$\leftarrow$ supernova SN 1006 (X-ray)

- Hypernovae: similar but 10-10 ${ }^{2}$ times more energetic; and portion of ejecta reaches $\geq$ semi-relativistic speed, possibly anisotropic

$\sim 500$ times the rate density of GRBs 


\section{Hypernova ejecta as UHECR sources}

(XY Wang et al, 2007, PRD 76:3009; Budnik et al, 2008, ApJ 673:928)

- Type Ib/c but isotropic equiv $\boldsymbol{E}_{\boldsymbol{H}} \sim 3-5 \times 10^{52}$ erg

- 500 times GRB rate, and $10^{-1}-10^{-2}$ usual SNIa rate

- Semi-relativistic ( $v \sim c$, or $\Gamma \beta \geq 1$ ) comp. in outflow (shock accelerates down the envelope gradient)

- Assume shock expands in WR progenitor wind, magnetic field fraction $\varepsilon_{\mathrm{B}}$ of equipartition

$$
B^{2} / 8 \pi=2 \epsilon_{B} \rho_{w}(R) c^{2} \beta^{2} \quad \rho_{w}(R) \propto R^{-2}
$$

Max. CR energy: $\quad \varepsilon_{\max } \simeq Z e B R \beta=4 \times 10^{18} Z$

$$
\times \epsilon_{B,-1}^{1 / 2}\left(\frac{v}{10^{10} \mathrm{cms}^{-1}}\right)^{2}\left(\frac{\dot{M}}{3 \times 10^{-5} \mathrm{M}_{\odot} \mathrm{yr}^{-1}}\right)^{1 / 2} v_{w, 3}^{-1 / 2} \mathrm{eV}
$$


Origin of $10^{19}-10^{21} \mathrm{eV}$ UHECR: may be GRB - but what about $10^{16}-10^{19} \mathrm{eV}$ ?

\section{HYPERNOVAE?}

- Radio, X-ray \& gamma-ray observations of SN1998bw/GRB980425 :

4 sub-energetic GRB - GRB980425: E 1e48 erg ( $d=38$ Mpc)

- Radio afterglow modeling: E>1e49 erg, $\rightarrow$ Gamma 1-2

- X-ray afterglow: E $\sim 5$ e49 erg, $\rightarrow$ beta $=0.8$

\section{$\rightarrow$ Mildily relativistic ejecta component}

$$
\begin{aligned}
& E_{\text {_SN }}=3-5 \mathrm{e} 52 \mathrm{erg} \\
& \mathrm{V}_{\text {avg }}=0.1 \mathrm{C}
\end{aligned}
$$

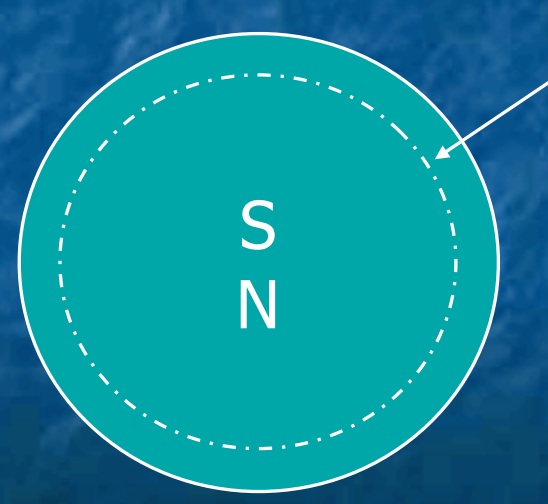

Other SN/GRB w. semi-relativistic ejecta: $\rightarrow$
SN shock acceleration in the Envelope? Tan et al. 01 Woosley et al. 99

- SN2003Iw/GRB031203

- SN2006aj/GRB060218 


\section{Maximum energy of accelerated particles}

1) Type Ib/c hypernovae expanding into stellar wind of WR star

2) equipartition magnetic field $B$, both upstream and downstream

$$
B^{2} / 8 \pi=2 \epsilon_{B} \rho_{w}(R) c^{2} \beta^{2} \quad \rho_{w}(R) \propto R^{-2}
$$

Maximum energy: Hillas $\quad$ Bell \& Lucek

$$
\begin{aligned}
& \varepsilon_{\max } \simeq Z e B R \beta=4 \times 10^{18} \mathrm{Z} \\
& \times \epsilon_{B,-1}^{1 / 2}\left(\frac{v}{10^{10} \mathrm{cms}^{-1}}\right)^{2}\left(\frac{\dot{M}}{3 \times 10^{-5} \mathrm{M}_{\odot} \mathrm{yr}^{-1}}\right)^{1 / 2} v_{w, 3}^{-1 / 2} \mathrm{eV}
\end{aligned}
$$

Protons can be accelerated to $\sim 10^{19} \mathrm{eV}$ Heavy nuclei can be accelerated to $\sim Z^{* 10^{19}} \mathrm{eV}$ 


\section{Flux level--- energetics}

Kinetic energy generation rate:

$\dot{\epsilon}_{k}(z=0)=R_{\mathrm{HN}} E_{k, \mathrm{HN}}$
$=2.5 \times 10^{46}\left(\frac{R_{\mathrm{HN}}}{500 \mathrm{Gpc}^{-3} \mathrm{yr}^{-1}}\right) \mathrm{erg} \mathrm{Mpc}^{-3} \mathrm{yr}^{-1}$

Compare w, normal GRBs

\begin{tabular}{|l|l|l|}
\hline & $\begin{array}{l}\text { Hypernova } \\
(\mathrm{V}=0.1 \mathrm{c})\end{array}$ & Normal GRBs \\
\hline $\begin{array}{l}\text { Rate } \\
(\mathrm{z}=0)\end{array}$ & $\sim 500 \mathrm{Gpc}^{-3} \mathrm{yr}^{-1}$ & $\sim 1 \mathrm{Gpc}^{-3} \mathrm{yr}^{-1}$ \\
\hline $\begin{array}{l}\text { kinetic } \\
\text { energy }\end{array}$ & 3-5e52 erg & 1e53-1e54erg \\
\hline
\end{tabular}

Normal Ib/c SN rate:

The required rate :

$\sim 2-5 \times 10^{4} \mathrm{Gpc}^{-3} \mathrm{yr}^{-1}$

$R_{\mathrm{HN}}=750 Z^{-1.2}\left(f_{z} / 3\right)^{-1} \mathrm{Gpc}^{-3} \mathrm{yr}^{-1}$

sub-energetic GRB rate:

$100-1800 \mathrm{Gpc}^{-3} \mathrm{yr}^{-1}$

Soderberg et al. 06 


\section{Energy distribution with velocity}

Data from Soderberg et al.

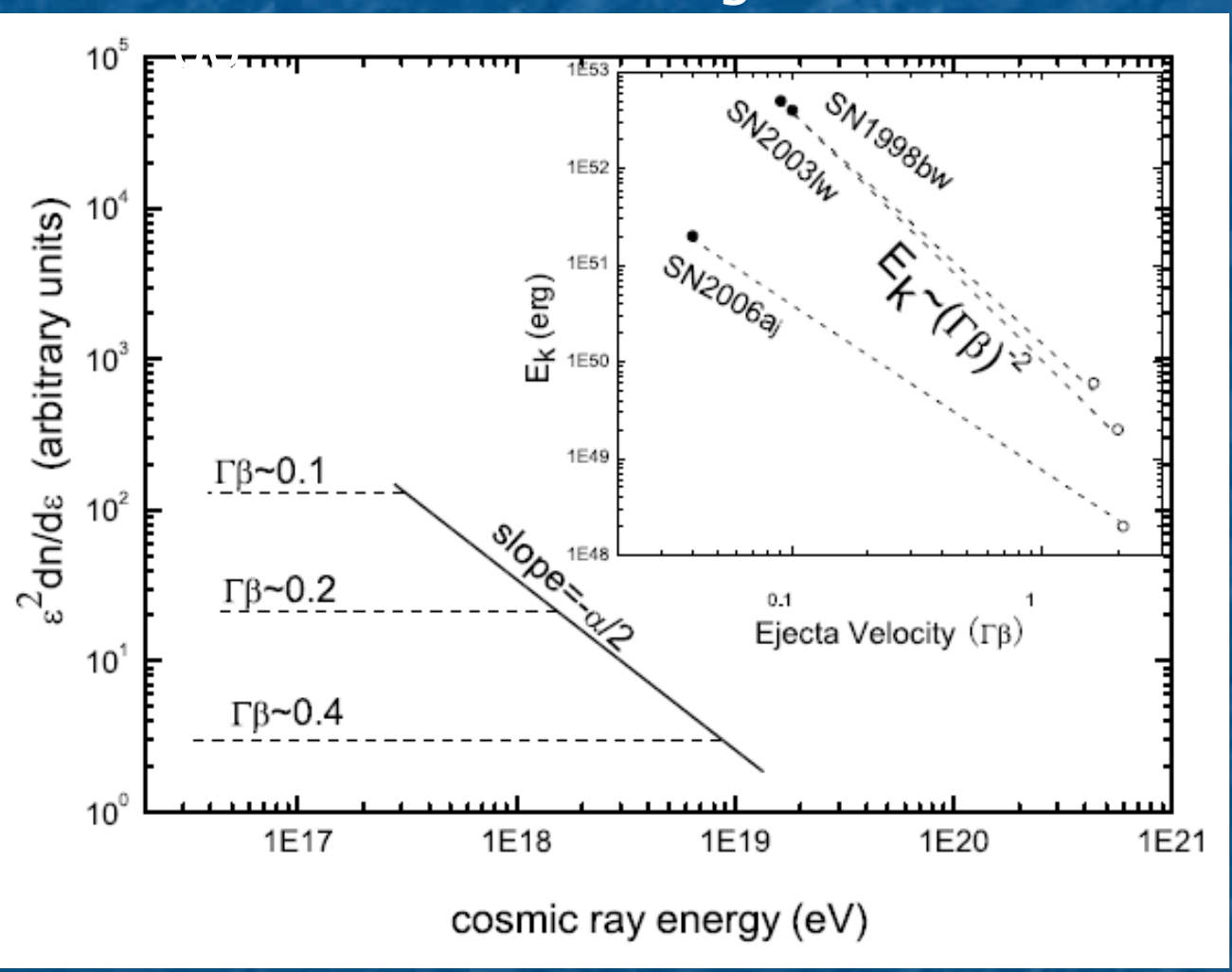

Wang, Razzaque, Meszaros, Dai 07
Normal SN $E_{k} \propto(\Gamma \beta)^{-5}$

Very steep distribution $\rightarrow>$ negligible contribution to high-energy CRs Berezhko \& Volk 04

Semi-relativistic hypernova: high velocity ejecta with significant energy

$E_{k} \sim(\Gamma \beta)^{-2}$

CR spectrum: 


\section{Energy distribution with velocity}

Data from Soderberg et al.

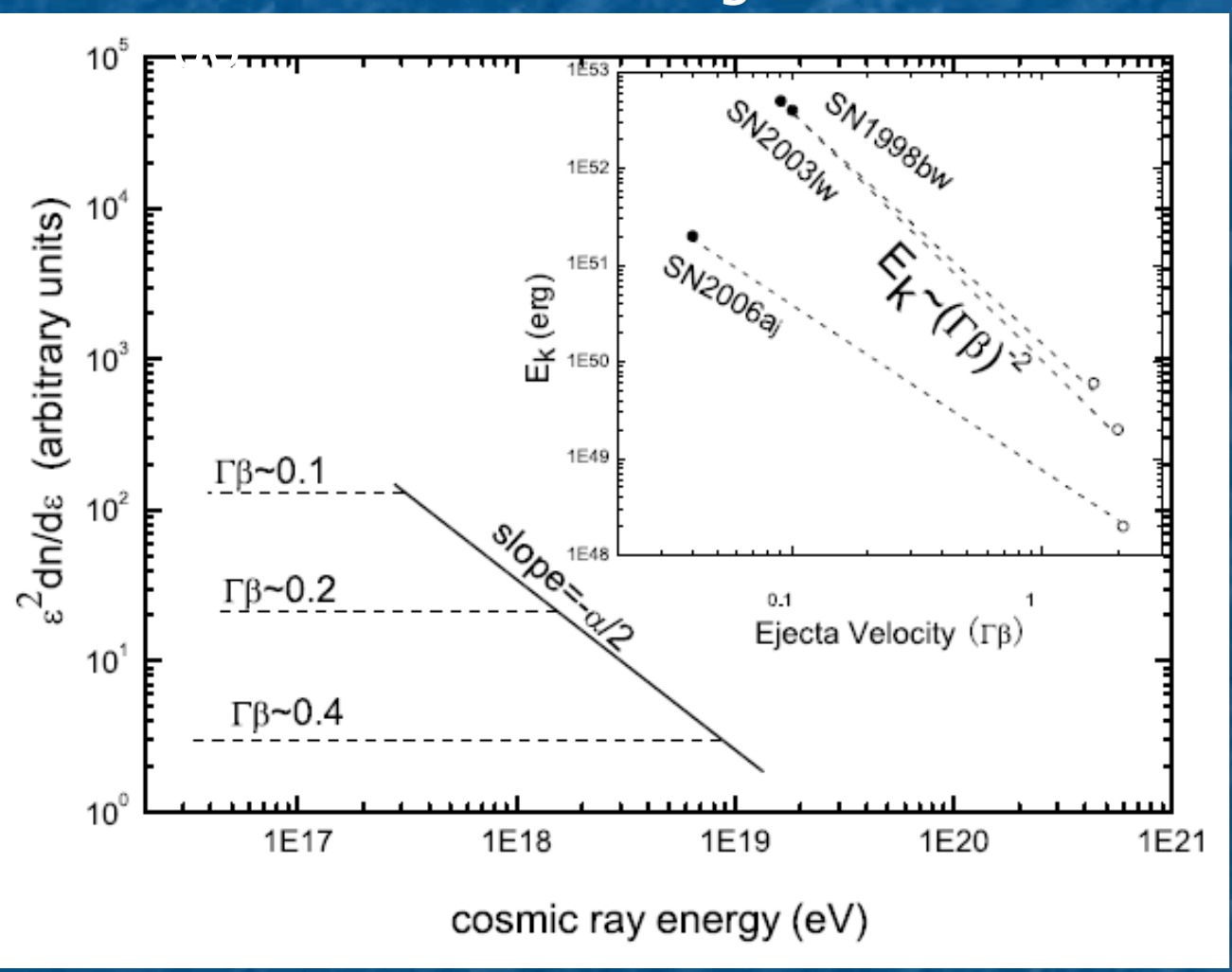

Wang, Razzaque, Meszaros, Dai 07
Normal SN $E_{k} \propto(\Gamma \beta)^{-5}$

Very steep distribution $\rightarrow>$ negligible contribution to high-energy CRs Berezhko \& Volk 04

Semi-relativistic hypernova: high velocity ejecta with significant energy

$E_{k} \sim(\Gamma \beta)^{-2}$

CR spectrum: 


\section{Transition from GCRs to EGCRs}

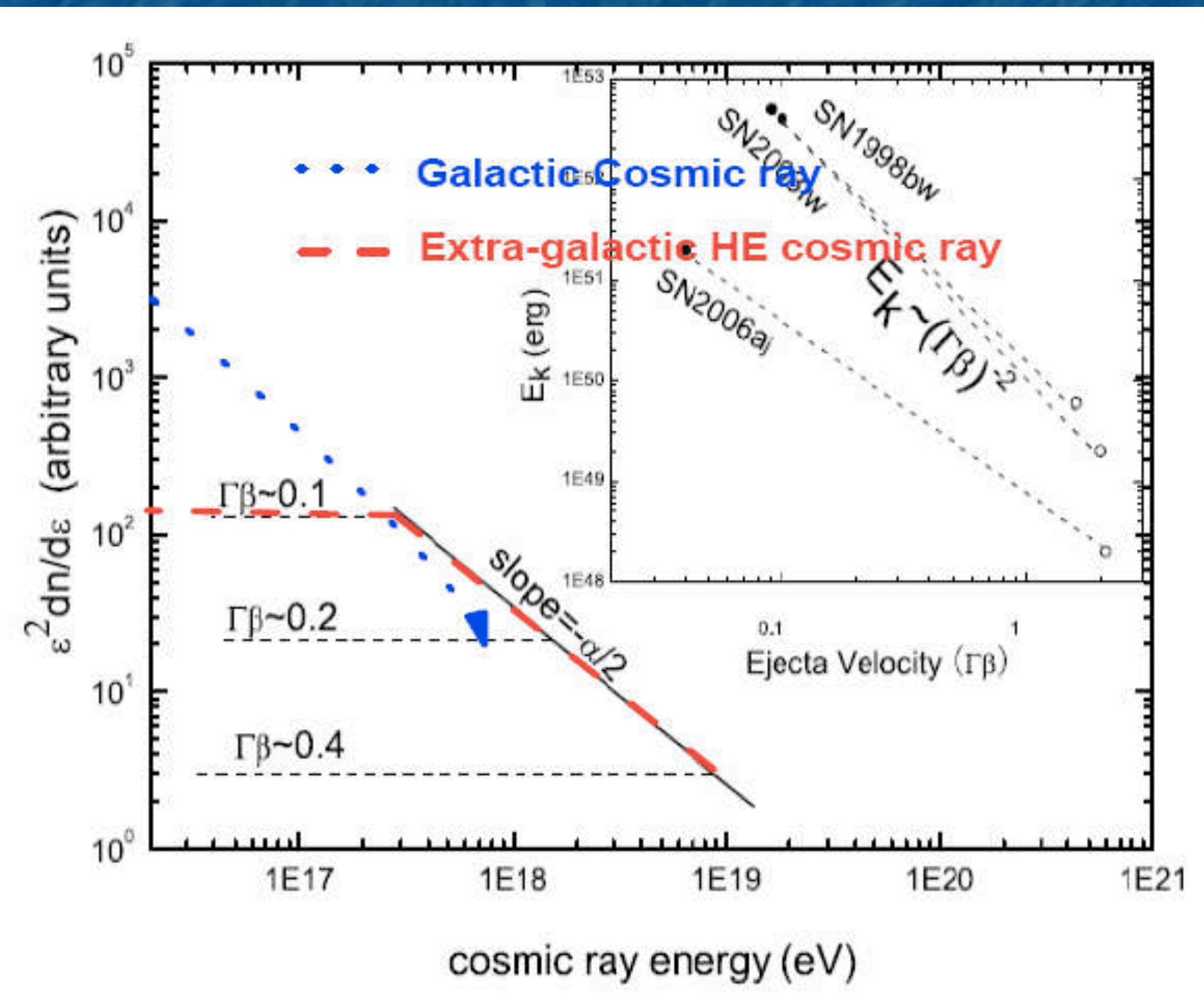




\section{What about Magnetars?}

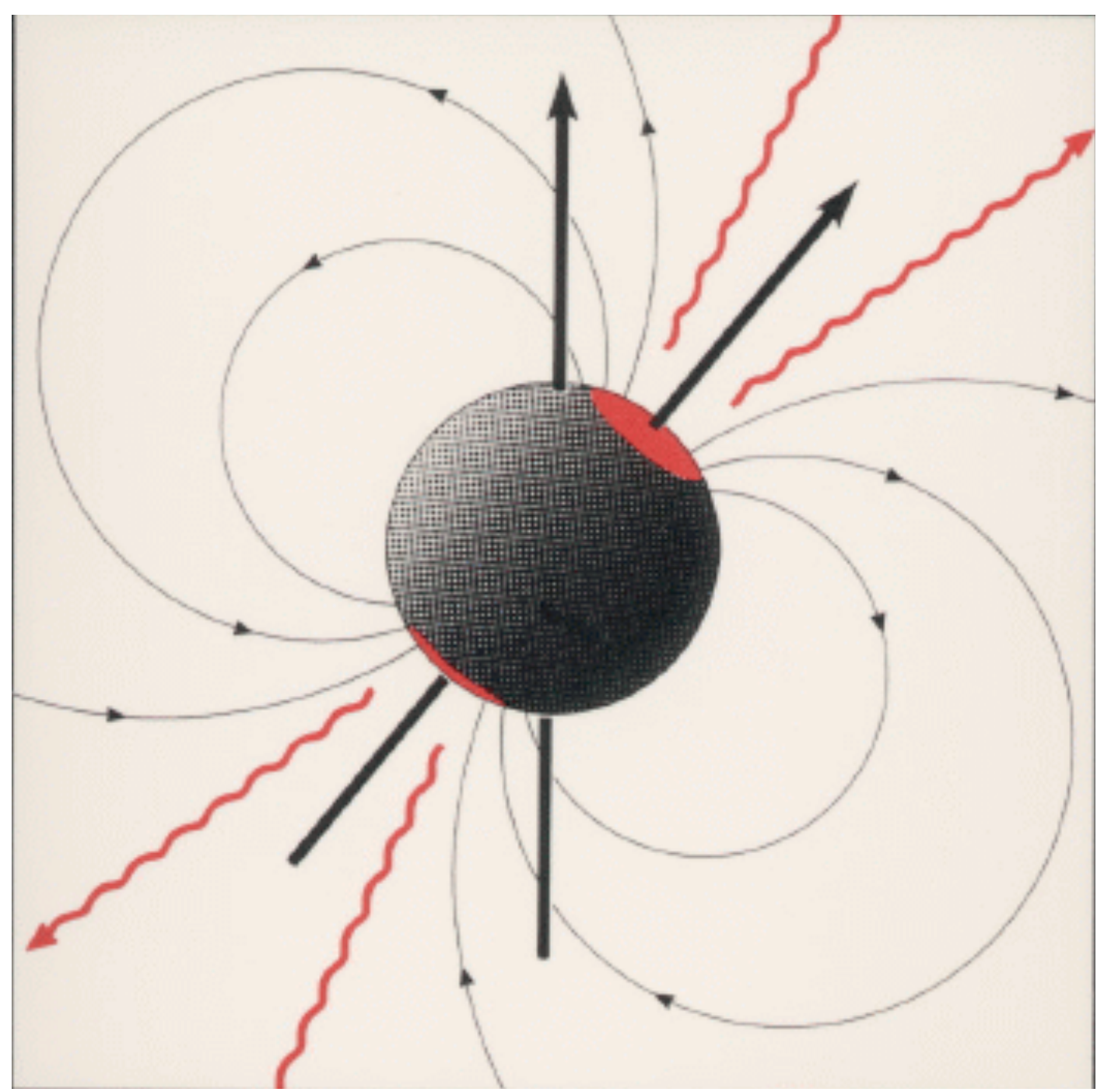

Pulsar: spinning neutron star: $\mathrm{R} \sim 10 \mathrm{~km}$ and magnetic field usually $\mathrm{B} \sim 10^{12} \mathrm{G}$;

but MAGNETAR: $B \sim 10^{14}-10^{15} \mathrm{G}$ (1010-1011 Tesla) 


\section{Magnetars as UHECR sources?}

- Surface magnetic field strengths $\boldsymbol{B}_{s} \sim 10^{14}-10^{15} G$ (whereas "normal" NS have only $\leqslant 10^{12}-10^{13} \mathrm{G}$ )

- Fraction $\leqslant 0.1 \mathrm{CC}$ SNe may result in magnetars

- Newly-born magnetar $\mathrm{R} \sim 10^{6} \mathrm{~cm}, \Omega=2 \pi / \mathrm{P} \sim 10^{4} \mathrm{P}_{-3}{ }^{-1}$

- Light-cylinder $\mathrm{R}_{\mathrm{LC}} \sim \mathrm{c} / \Omega \sim 5 \times 10^{6} \mathrm{P}_{-3} \mathrm{~cm}$ ( $\rightarrow$ accel.)

- $\mathrm{B}_{\mathrm{LC}} \sim \mathrm{B}_{\mathrm{s}}\left(\mathrm{R}_{\mathrm{s}} / \mathrm{R}_{\mathrm{LC}}\right)^{3} \sim 10^{13} \mathrm{P}_{-3}{ }^{-3} \mathrm{G}$

$\rightarrow \mathrm{E}_{\max } \sim \mathrm{ZeBR}(\mathrm{v} / \mathrm{c}) \sim 10^{21} \mathrm{Z} \mathrm{B}_{\mathrm{s}, 15} \mathrm{P}_{-3}{ }^{-2} \mathrm{eV}$

Or: In PNS wind, wake-field acceleration can lead to UHECR energies $E(t) \leqslant 10^{20} \mathrm{eV} \mathrm{Z} \mathrm{\eta -1} \mu_{33^{-1}} \mathrm{t}_{4}{ }^{-1}$ 


\section{One possible type of}

GZK UHECR/UHENU astrophysical source 


\section{Standard(+) Model of GRB}

(as UHECR/NU source)

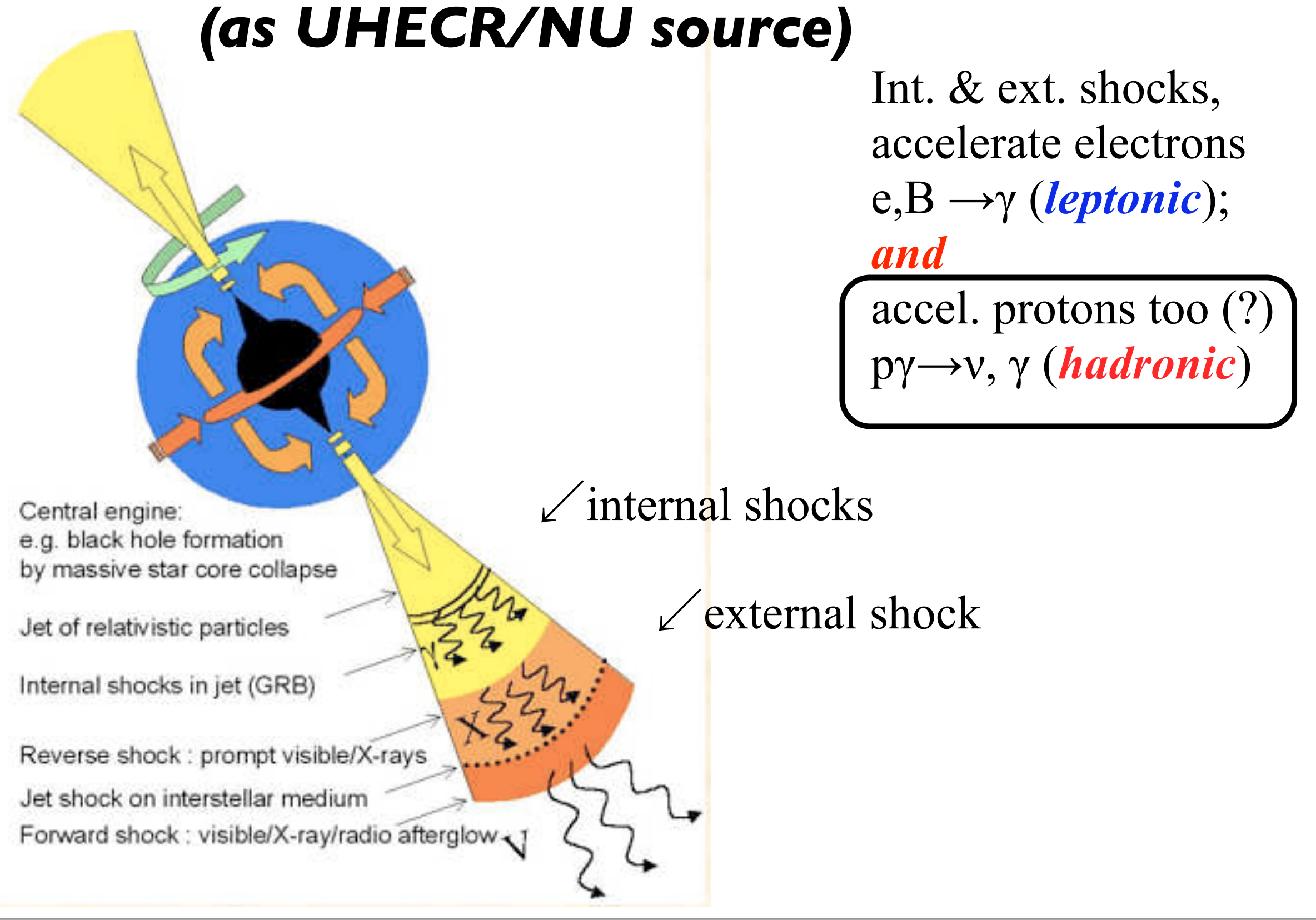




\section{GRB VHE neutrinos}

At the simplest level:

- $R_{d}, R_{p h}$ : dissipation radius where particles accelerated

- Fermi (or mag, reconn.) accel. $d N_{p, e} / d E \sim E^{-q}$

- $\mathrm{e}^{ \pm}, \mathrm{B} \rightarrow \mathrm{\gamma}$

- $\mathrm{P}, \gamma \rightarrow \pi^{ \pm} \rightarrow \mu^{ \pm}, V_{\mu} \rightarrow \mathrm{e}^{ \pm}, \nu_{\mathrm{e}}, \mathrm{V}_{\mu}$

- For PL $\mathrm{dN} N_{e, p} / d E$ and $d N_{\gamma} / d E \rightarrow d N_{v} / d E$ also PL

- Parameters: $\epsilon_{\mathrm{p}}, \epsilon_{\mathrm{e}}, \epsilon_{\mathrm{B}}$ : energy ratios of $\mathrm{p}, \mathrm{e}, \mathrm{B}$ to $E_{\text {tot }}$

- E $E_{\text {tot: }}$ total burst energy, Г: bulk Lorentz factor 


\section{Simple $p, \gamma \rightarrow \Delta \rightarrow \ldots$ model}

$\mathrm{p} \gamma \rightarrow \Delta$ interaction of a Band- $\gamma$ spec. with an $\mathrm{E}^{-2}$ proton spec. is

(WB 97)
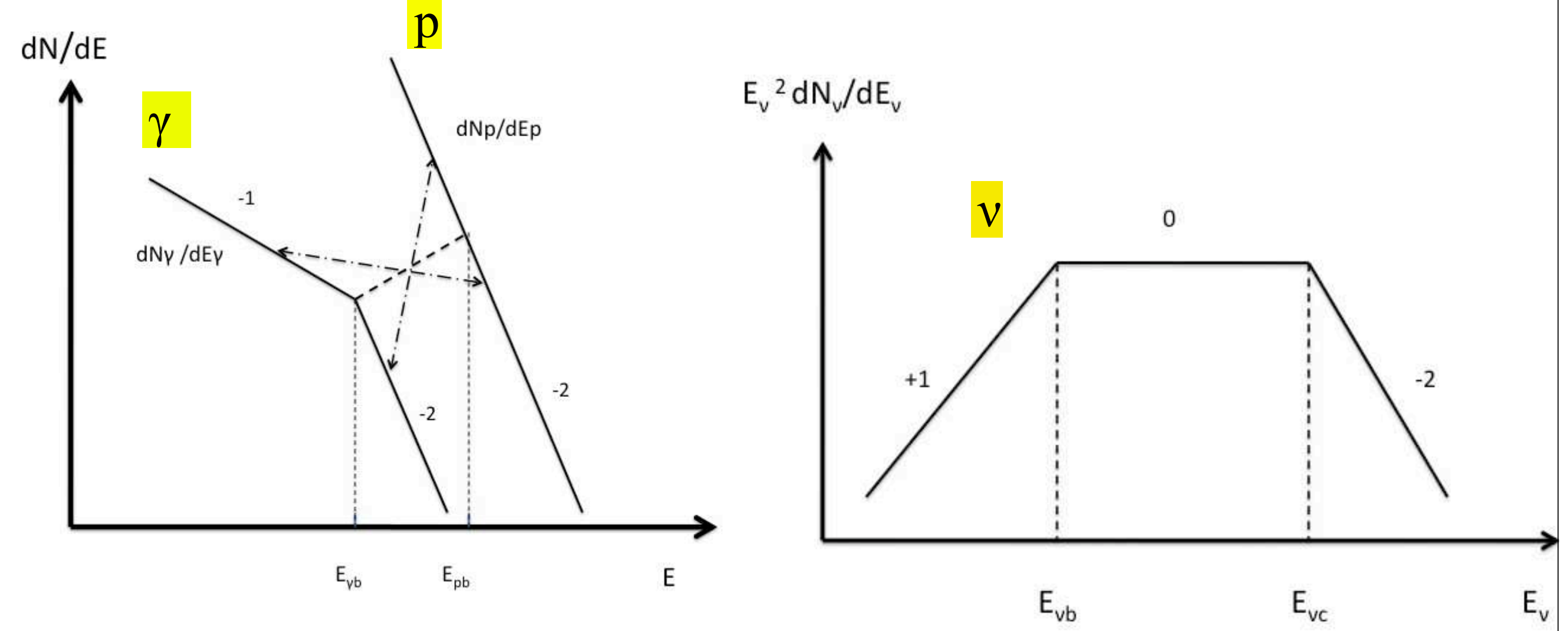


\section{Vs from py from int. \& ext. shocks}

NOTE: internal shock (old paradigm ) + simple $\Delta$-res. approx.

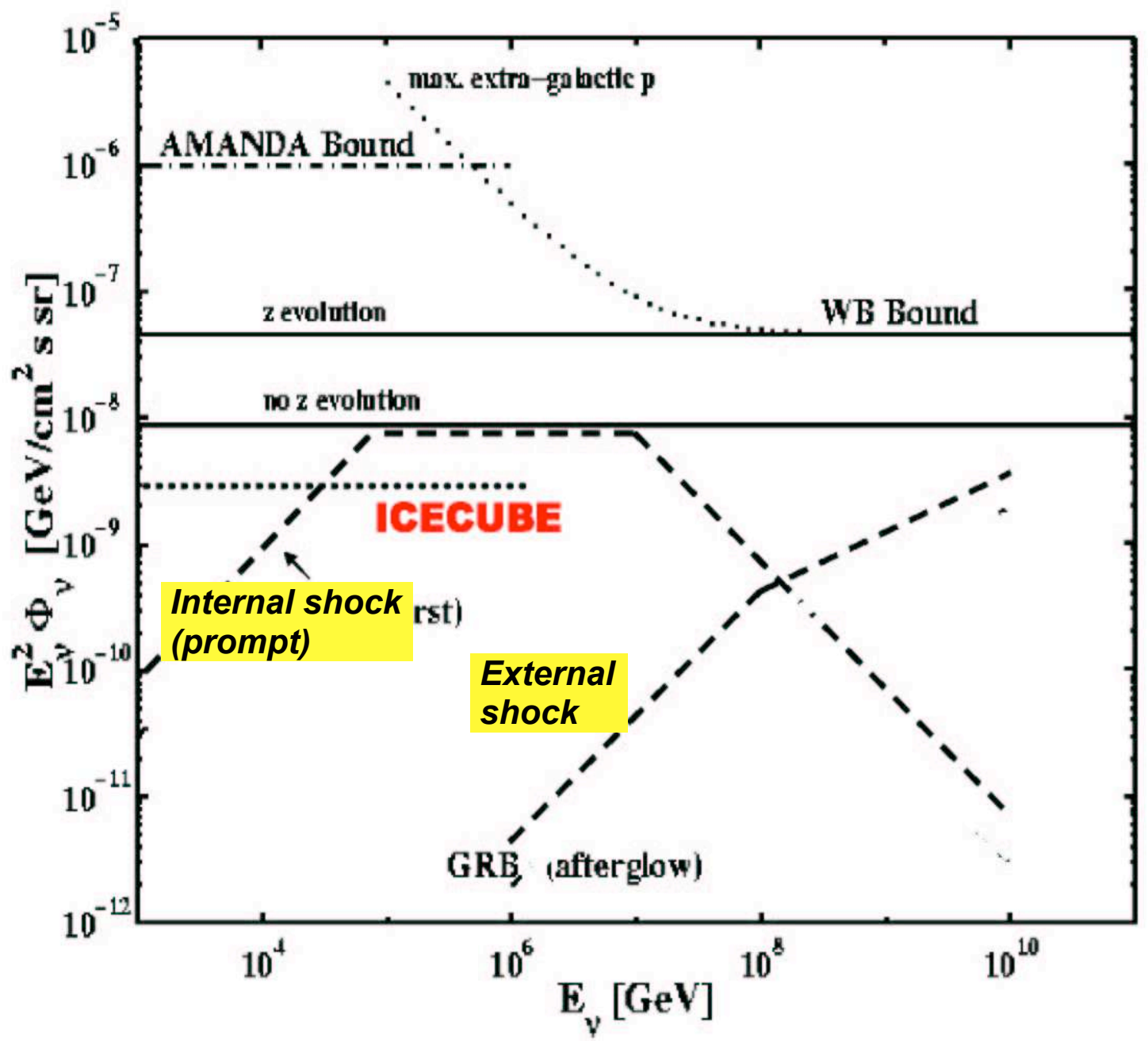

- $\Delta$-res.: $\mathrm{E}_{\mathrm{p}}^{\prime} \mathrm{E}_{\mathrm{\gamma}}^{\prime} \sim 0.3 \mathrm{GeV}^{2}$ in comoving frame, in lab:

$\rightarrow \mathrm{E}_{\mathrm{p}} \geq 3 \times 10^{6} \mathrm{\Gamma}_{2}^{2} \mathrm{GeV}$

$\rightarrow \mathrm{E}_{v} \geq 1.5 \times 10^{2} \Gamma_{2}^{2} \mathrm{TeV}$

- Internal shock $p \gamma_{\mathrm{MeV}}$

$\rightarrow \sim 100 \mathrm{TeV} \nu \mathrm{s}$

- ( External shock pyuv

$\rightarrow \sim 0.1-1 \mathrm{EeV} \nu \mathrm{s}$ )

- Diffuse flux: det.w. $\mathrm{km}^{3}$ 


\section{Data: IC40+59 search for VHE nus from 190 GRB (105 northern)}

Nature 484:351 (2012), the Icecube collab.; Abbasi and 242 others (incl. P.M.)

(A) Model dependent search

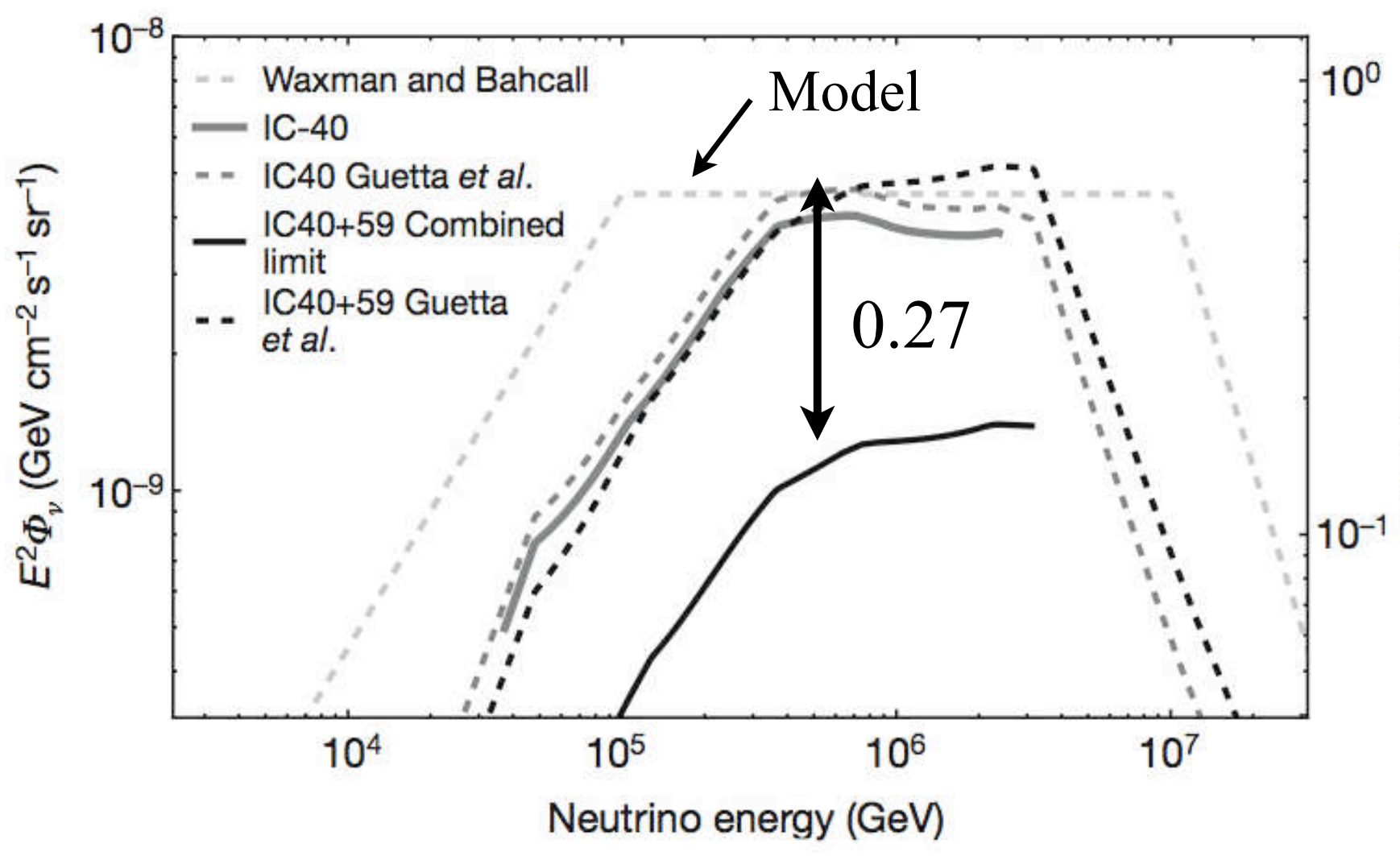

Data is factor 0.27 below model $\mathrm{F}_{v}$ at $90 \% \mathrm{CL}$
- Analyze 190 GRBs localized w. $\gamma$-rays betw, $\mathrm{T}_{\text {start }} \& \mathrm{~T}_{\text {end }}$

- Use the WB'97 and Guetta'04 proton acceleration model in internal shocks, with $\mathrm{E}^{-2} \operatorname{spectr}, \varepsilon_{\mathrm{p}} / \varepsilon_{\mathrm{e}}=10$, and $\mathrm{p} \gamma \rightarrow \Delta$-res $\rightarrow v_{\mu}$

- Nu-flux normalized by obs. $\gamma$-ray flux, get $\mathrm{F}_{v}$ for 190 (right axis), and diffuse flux (all) assuming $677 \mathrm{yr}^{-1}$ 


\section{Model-independent ULs}

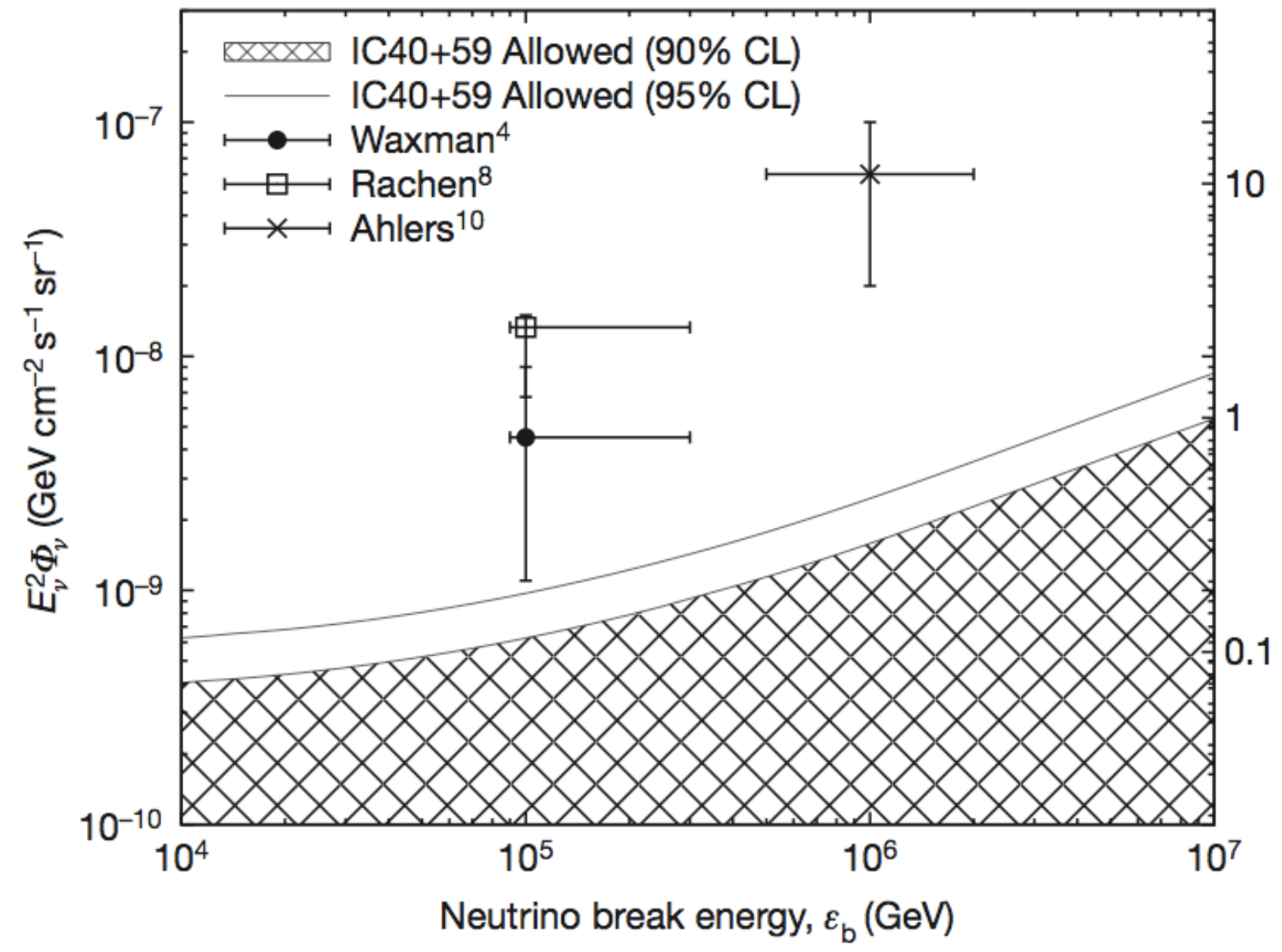

- $\quad$ Take observed $\gamma$-ray flux and $\gamma$-ray spectral break energy

- Use these to infer the $V$ flux and the $v$ spectral break energy

- The V-flux UL implies a UHECR proton flux UL, given by hatched region

- But (internal shock) models capable of explaining observed UHECR flux (data pts) are above this UL 


\section{IC59 2-year conclusions (190 GRBs)}

Nature 484:351 (2012)

- The fireball (more accurately: internal shock) model overpredicts the TeV-PeV nu-flux by a factor 3.7, (asuming $\mathrm{L}_{\mathrm{p}} / \mathrm{L}_{\mathrm{e}}=10, \Delta$-res only, Lorentz $\Gamma \sim 300-600$ )

- For a model independent fit, the 95\% CL nu-flux UL is $2-3 \sigma$ below what would be expected if the $G R B S$ contribute most of $G Z K$ UHECR flux.

- In these models, either $\boldsymbol{L}_{\boldsymbol{p}} / \boldsymbol{L}_{\boldsymbol{e}}$ must be substantially below factor 3-10 assumed here, or the production efficiency of neutrinos is lower than was assumed. 


\section{A significant achievement:}

- These are conservatively stated conclusions

- The first time VHE nus have put a significant constraint on a well-calculable astrophysical UHECR-UHENU model, at 90-95\% $\boldsymbol{C L}$

- This is very valuable

- Icecube is doing exciting astrophysics

- A significant first step towards $G Z K$ Khysics 


\section{But, on closer look at IC3 analysis...}

(Li '12, PRD 85:027301)

- IC22-59 analysis used a simplified version of WB97, which results in overestimated model nu-fluxes

- Assumed $\mathrm{F}_{v}{ }^{\mathrm{IC}} / \mathrm{F}_{\mathrm{p}}=(1 / 8) \mathrm{f}_{\pi, \mathrm{b}}$, where $1 / 8$ because $1 / 2 \mathrm{p} \gamma$ lead to $\pi^{+}$and each $v_{\mu}$ carries $1 / 4 E_{\pi}$, and $f_{\pi, b}=f_{\pi}\left(E=E_{b}\right)$

- But $\mathrm{f}_{\pi}(\mathrm{E})=\mathrm{f}_{\pi, b}$ is OK only for $\mathrm{E}_{\mathrm{b}}<\mathrm{E}<\mathrm{E}_{\pi, \text { cool }}$;

- Also for $\mathrm{E}<\mathrm{E}_{b}$, have $\mathrm{f}_{\pi} \propto \mathrm{E}$, because of decr. \# of photons

- Result: model $v_{\mu}$ flux overestimated by factor $\sim 5$ (at least)

- In addition, ignored multipion, Kaon decay, etc. 


\section{In greater detail..}

Even using the (old paradigm) int. shock model, the simplifications have an effect

(Hümmer et al '11, PRL 108:231101)
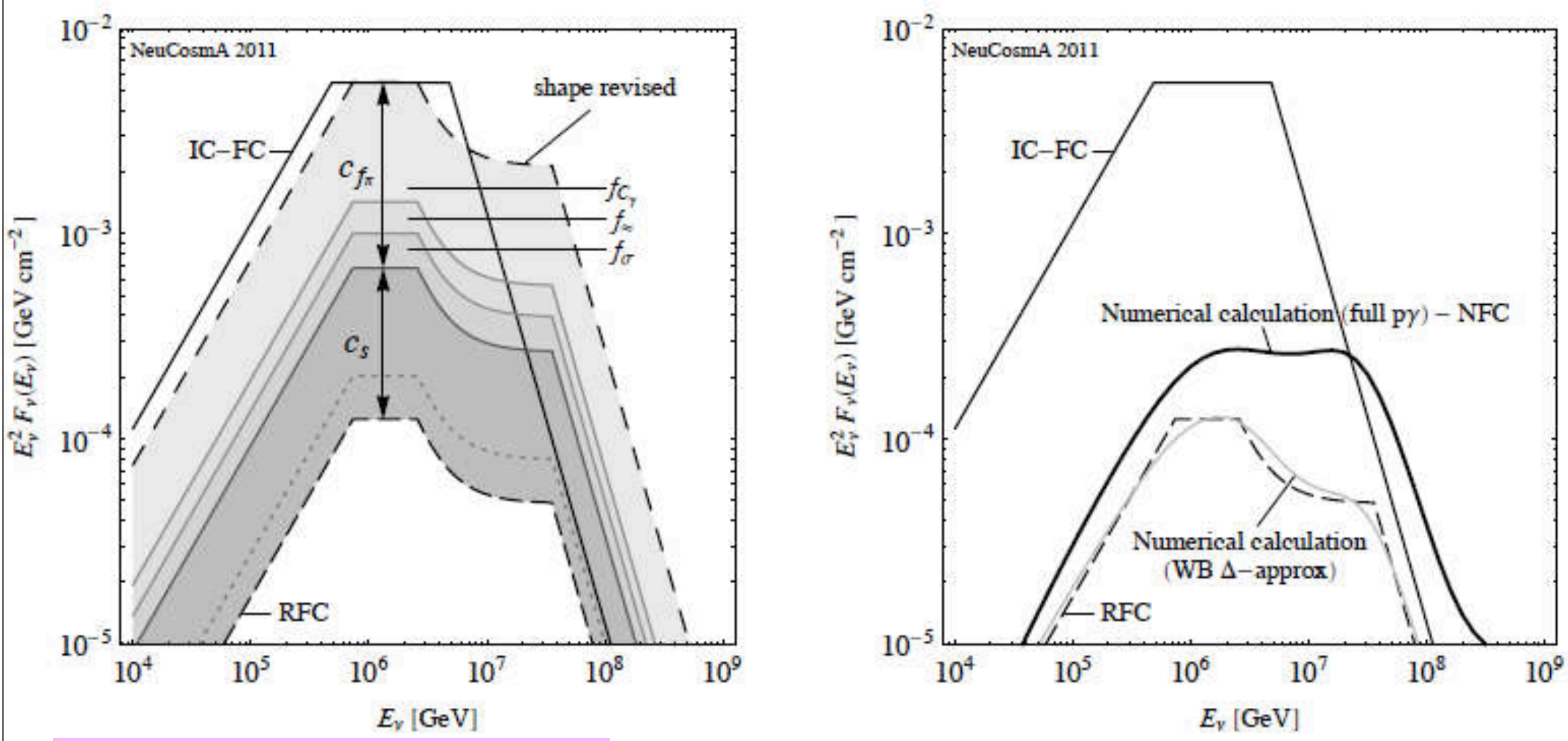

- IC-FC: IceCube Fball. Calc.

- $\mathrm{f}_{\mathrm{C}}$ : energy of all photons approx. by break energy

- RFC: "Revised" Fball.Calc. $\Rightarrow$

- $\mathrm{f}_{\approx}: 0.7$ (rounding error in one eq.)

- NFC: Numerical Fball.Calc.

- $\mathrm{f}_{\sigma}: 2 / 3$ (negelected width of $\Delta$-res.)

- $\mathrm{C}_{\mathrm{s}}$ : correct en. loss of second's \& E-dep of proton mfp 


\section{So for stand. IS model ....}

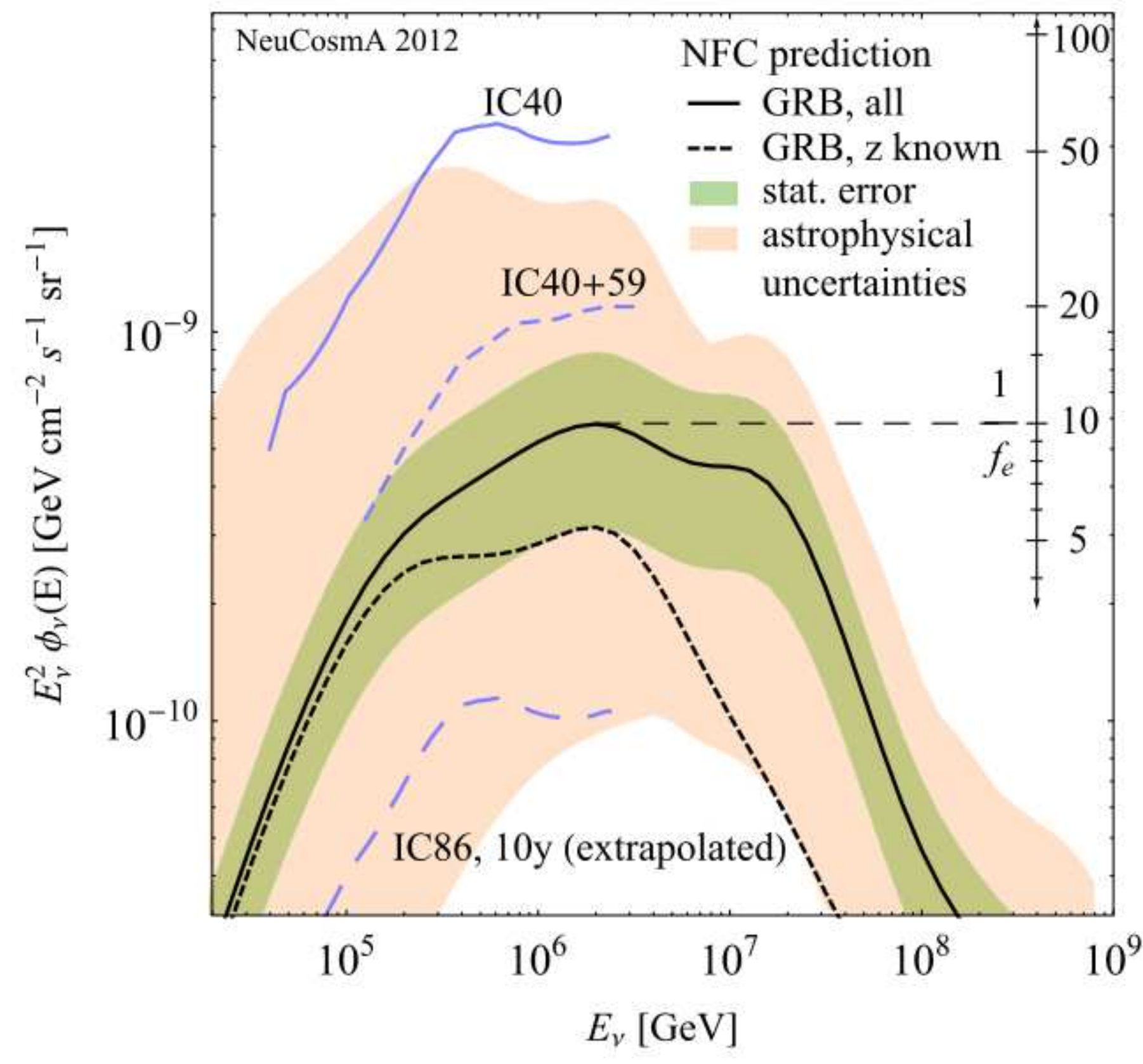

- Even using the old standard paradigm of internal shock, but with more detailed physics (incl. multipion and Kaon production, $\neq$ cool'g. break for $\pi$, $\mu$, and numerical as opposed to analytical calcul.)

- $\Rightarrow F_{v}$ predictions below IC40+59!

( $\leftarrow$ Hümmer et al, '11, PRL 108:231101)

(Also:

Li, '12, PRD 85:027301 ; He, et al '12, ApJ 752:29)

69 


\section{Furthermore, note that .....}

- Internal shock model is expedient: it is the best documented so far, and easy to calculate $\Rightarrow$ its use is widespread

- But ... int. shocks known (past 10 years) to have difficulties for gamma-ray phenomenology (efficiency, spectrum, etc)

- And, acceleration rate of protons vs. electrons is unknown; are protons injected into accel. process at $=$ or $\neq$ rate as electrons? Only energy restriction on model is $\boldsymbol{L}_{p} / \boldsymbol{L}_{\boldsymbol{e}} \leqslant \mathbf{1 0}$.

- Even if GRB are not GZK sources, model indep. searches leave the interesting possibility of lower, but observable neutrino fluxes from GRB

- AND: Alternatives to int. shock are being investigated (less easy to calculate; e.g. photospheric \& hadronic models) 


\section{Photospheric \& internal shock GRB models}

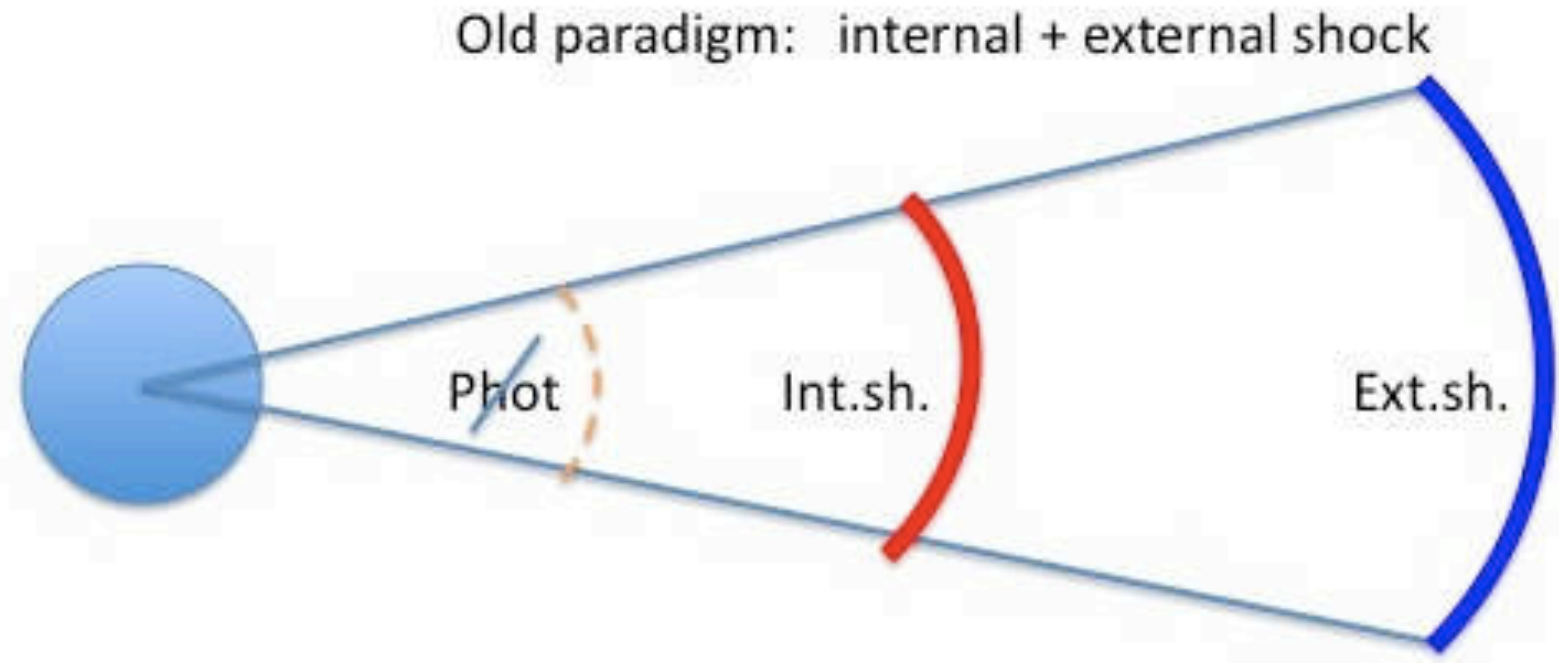

New paradigm: photosphere + external shock

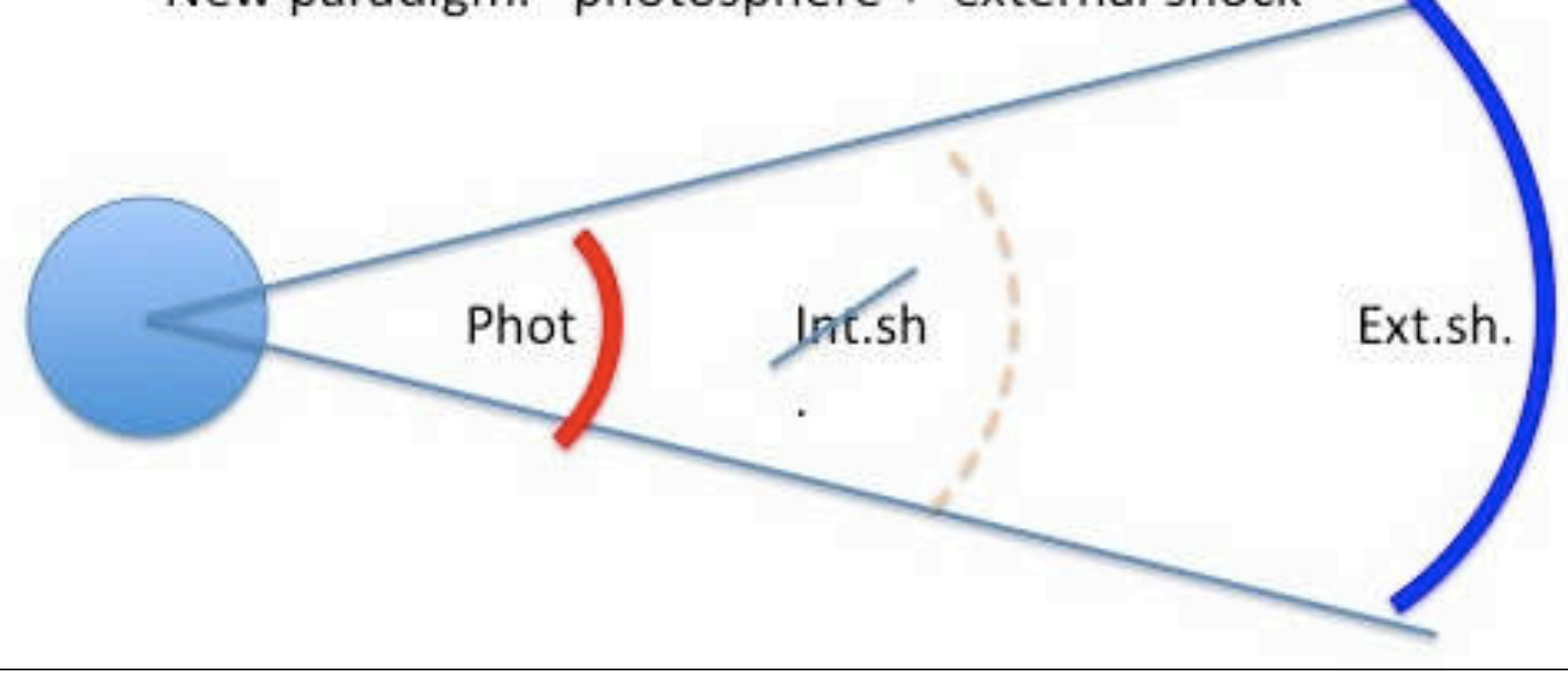




\section{Other GRB dynamics subtlety:}

$\Gamma(\mathrm{R})$
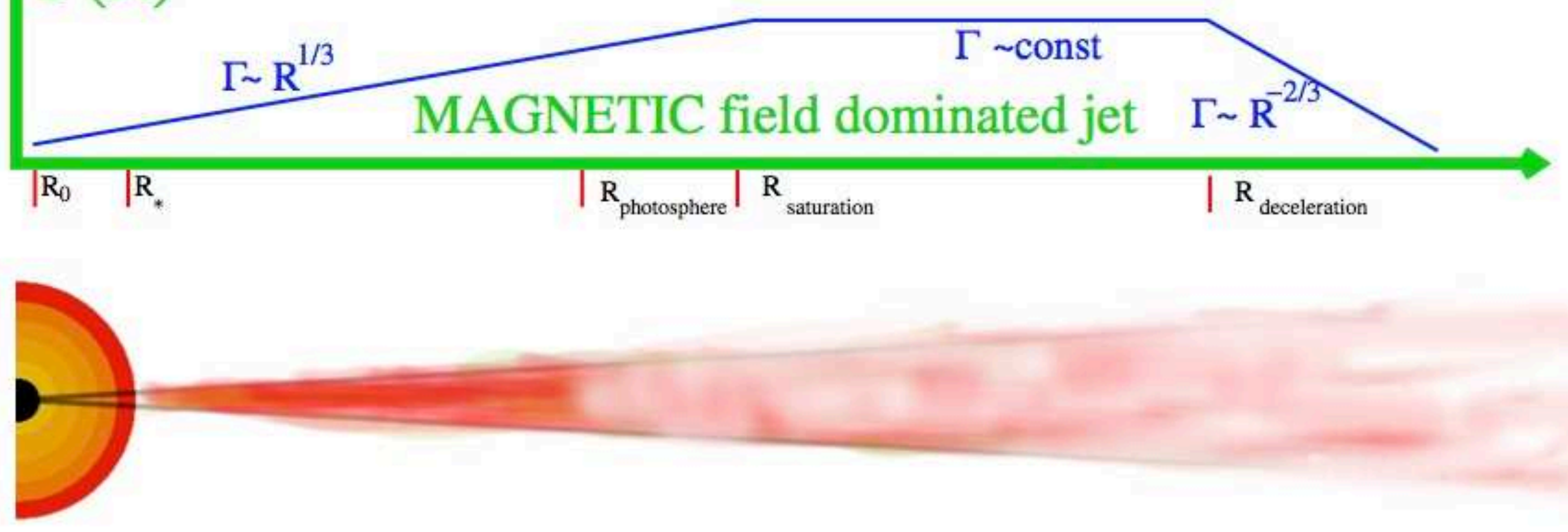

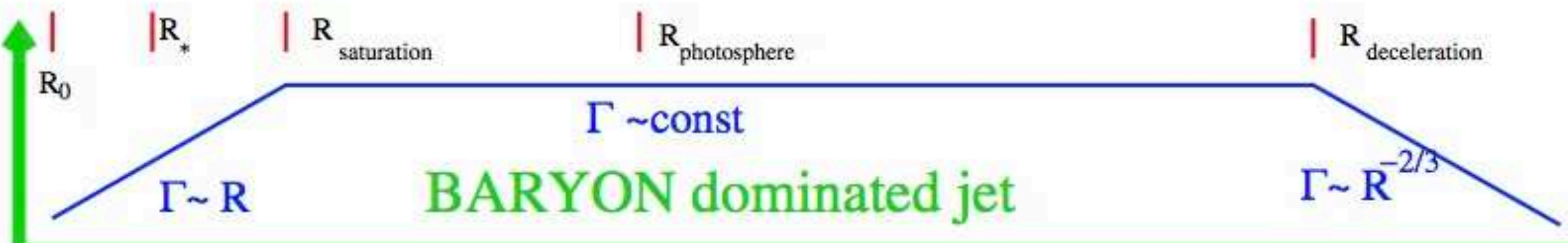




\section{$v_{\mu}$ from magn.dissip.phot. + ext.sh.}

Gao, Asano, Mészáros '12, JCAP 11:058

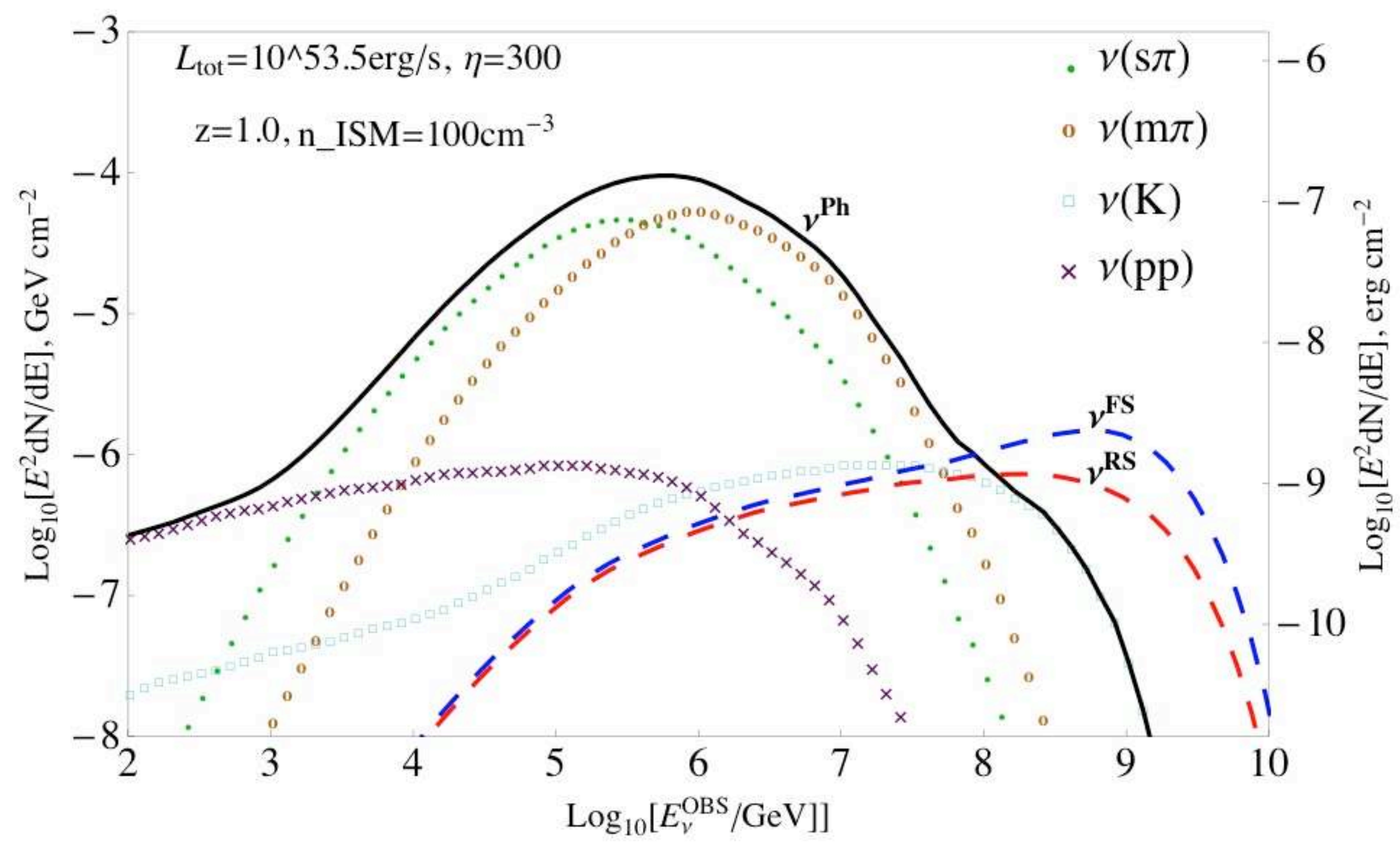




\section{Diffuse nu from MPh, Bph, IS, $\eta=300 \&$ IC3 lim.}

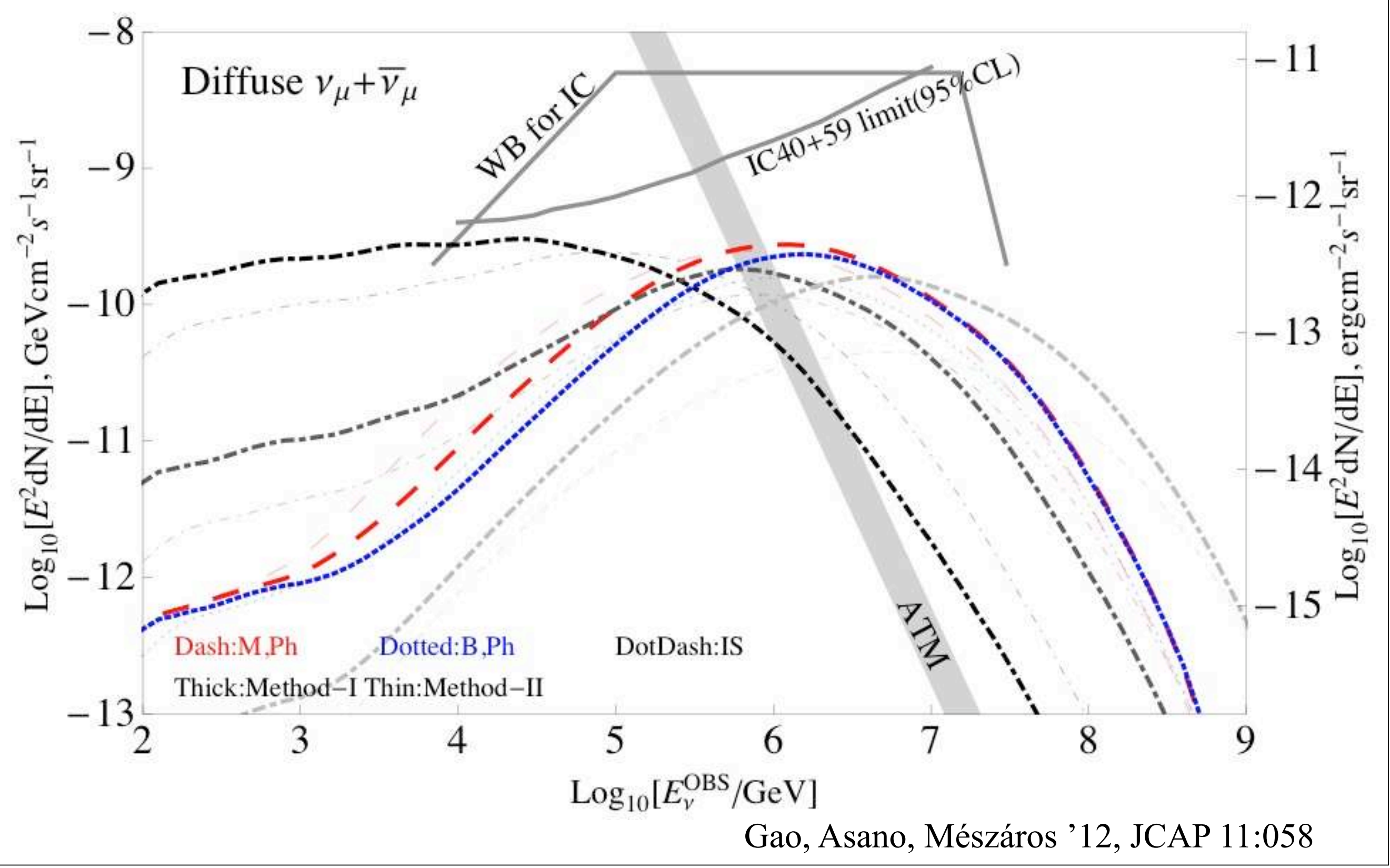




\section{Recent IC3 results on IS\& Phot. GRB}

33RD INTERNATIONAL COSMIC RAY CONFERENCE, RIO DE JANEIRO 2013

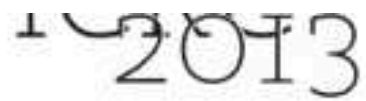

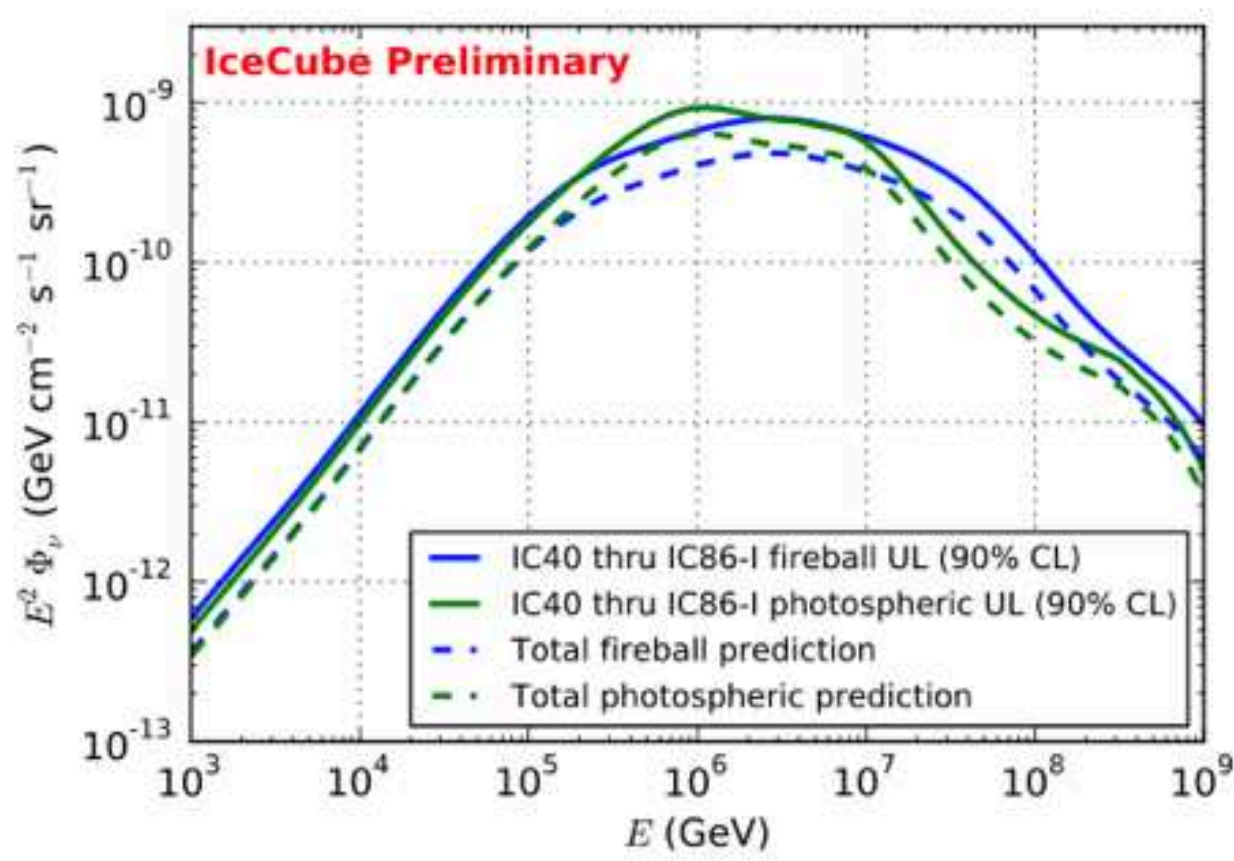

Figure 3: Fireball and photospheric model quasi-diffuse flux predictions and $90 \%$ CL upper limits from the combined analysis of four years of IceCube data. Full systematic treatment is deferred to a later publication, so these limits include an assumed $6 \%$ systematic uncertainty, which is the estimated uncertainty in the most recently published analysis. The fireball and photospheric model limits are 1.72 and 1.47 times the model predictions, respectively, so these models are not yet constrained by our results.

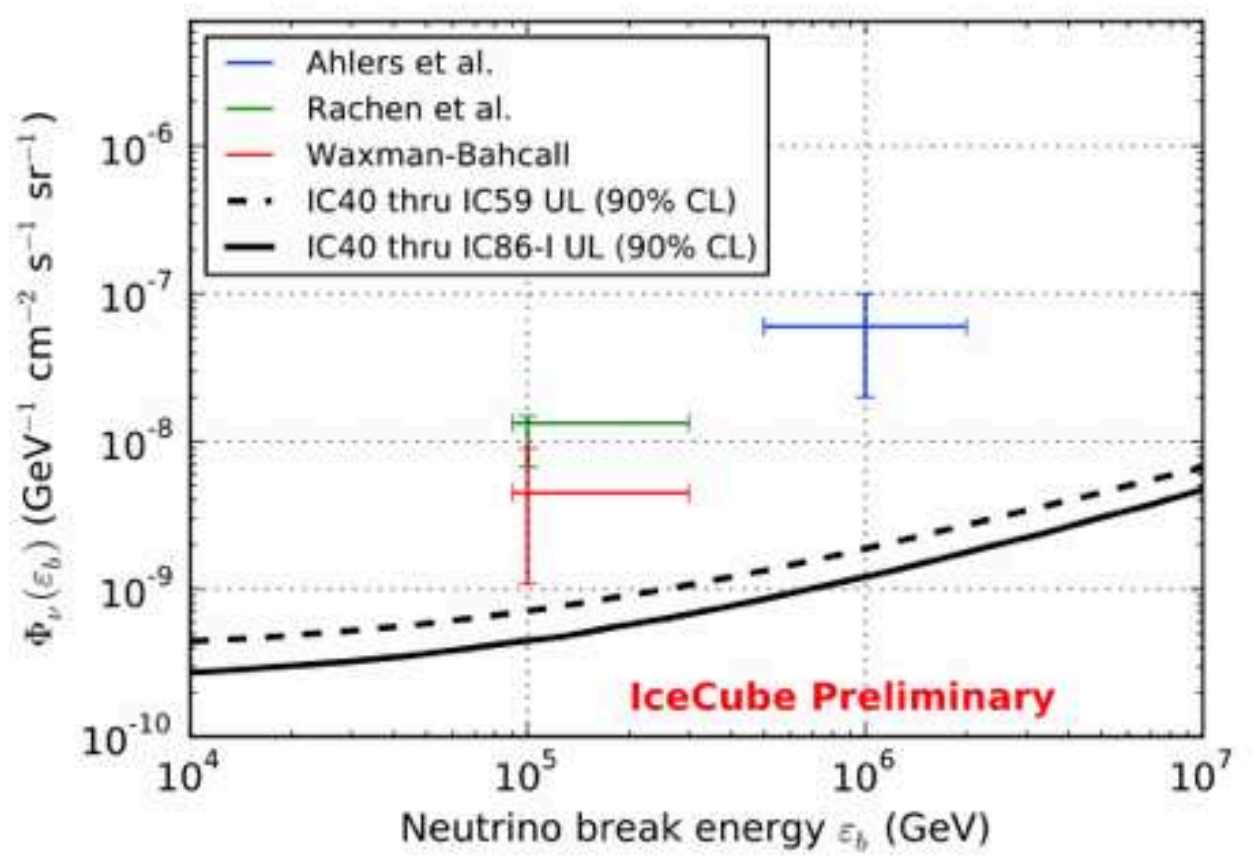

Figure 4: Compatibility of some models [20, 21, 22] of cosmic-ray-normalized neutrino fluxes with observations. The 90\% CL upper limits from the published IC40+IC59 analysis [2] as well as the new four year analysis are shown in comparison with some model predictions indicated as points with error bars. Without modification, these models are excluded by our results. 


\section{What about nus from "nearby monster" GRB I30427A?}

- At $\mathrm{z}=0.34$ and $\mathrm{L}_{\text {iso }} \sim 2 \times 10^{54} \mathrm{erg}, \mathbf{G R B} / \mathbf{3 0 4 2 7 A}$ was expected to be best candidate for nu-detection

- But, IceCube : No Detection! (GCN I4520)

- Is this suprising?

- At least 2 reasons why not:

I. Expect no V-detection in standard int. shock for this GRB (in IC40+59)

2. Expect no $V$-detection in one out of two other 'non-standard' models for this GRB (in IC40+59) 


\section{Back to GRB I 30427A: nus?}

\section{("Best", brigtest, nearest single candidate)}

Gao, Kashiyama, Mészáros 2013,ApJ, 772:L4
Calc. ULs using all recent corrections to physics
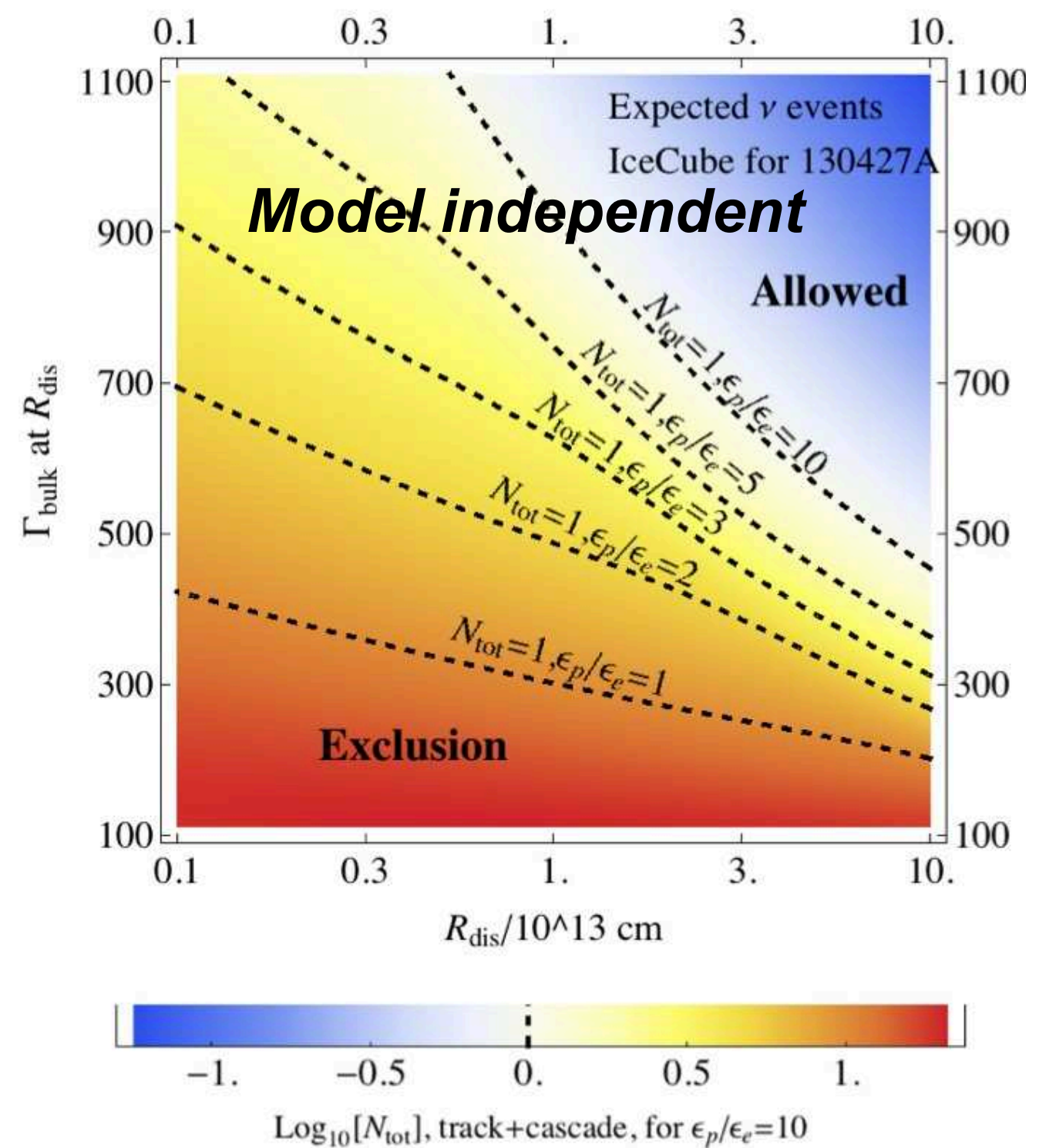

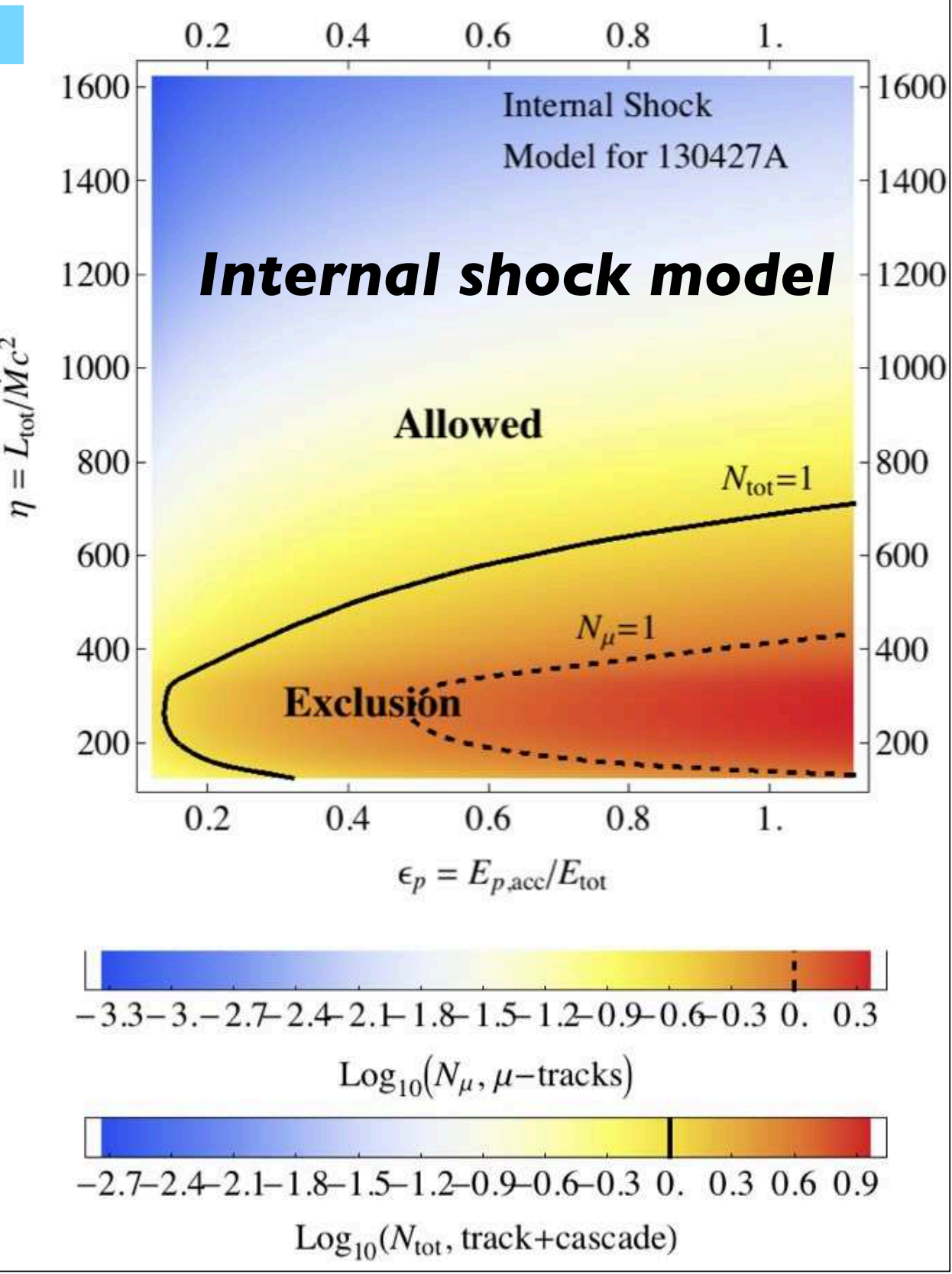




\section{30427A baryonic \& magnetic photosph. model}

Gao, Kashiyama, Mészáros 2013,ApJ, 772:L4
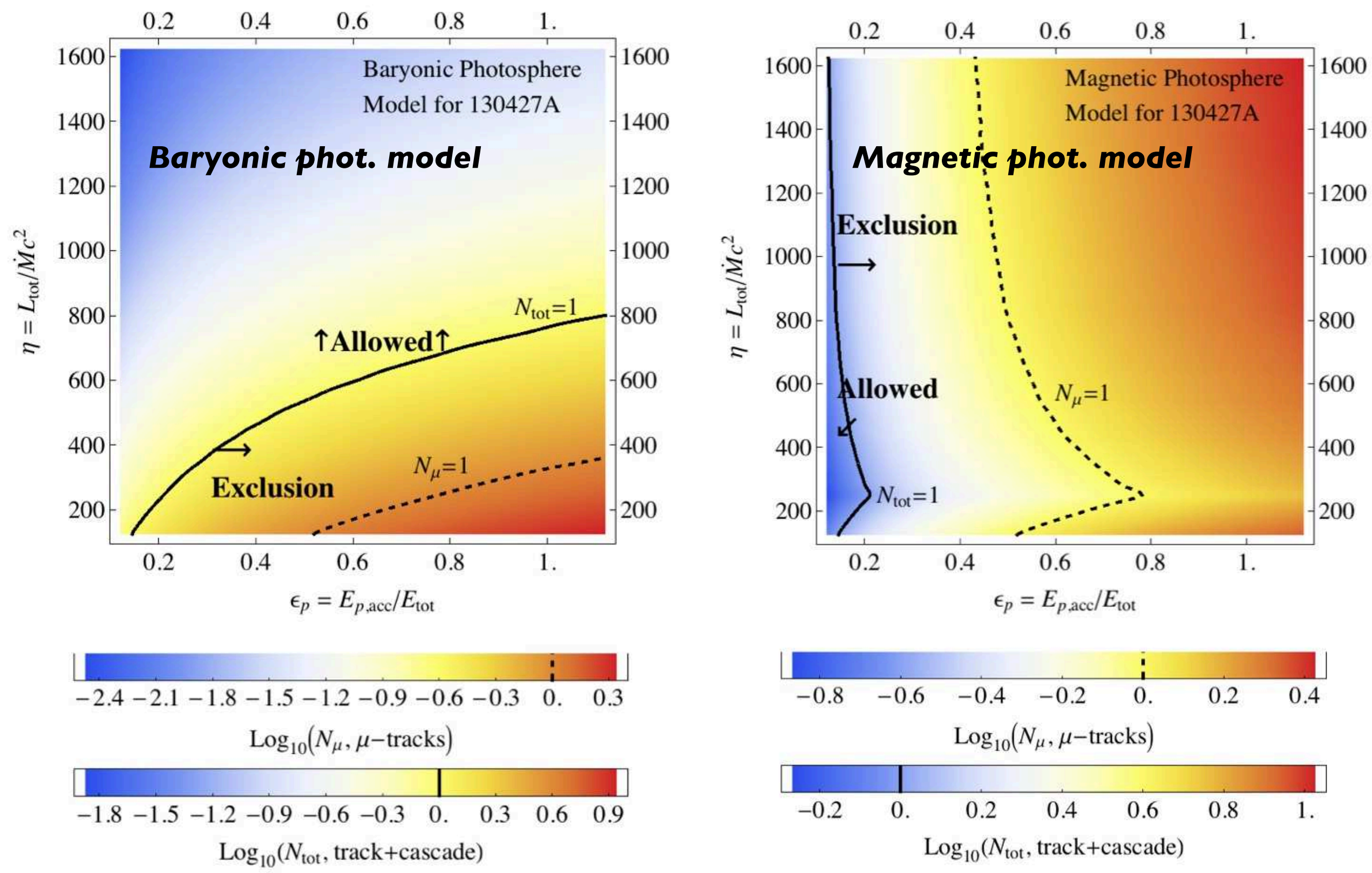


\section{That is:}

- IceCube neutrino ULs on the diffuse nu-bkg do not constrain the (fixed radius, i.e. steady state) IS model so far; will need several more years to get near the ULs

- IceCube ULs on GRBI30427A do not constrain either the IS or baryonic phot. models, but do constrain mag. phot. model

- Will need consider better, time-dep models incl. effects of pair formation for model fits 


\section{A more accurate \\ Internal Shock GRB CR/nu calculation:}

- All previous GRB neutrino/CR calculations were time-independent (steady-state)

- Now can go one better on that, and do time-dependent 


\section{Time-dependent CR escape,}

gamma-rays \& neutrinos

Asano \& Mészáros, ApJ acc., arX: 1402.6057

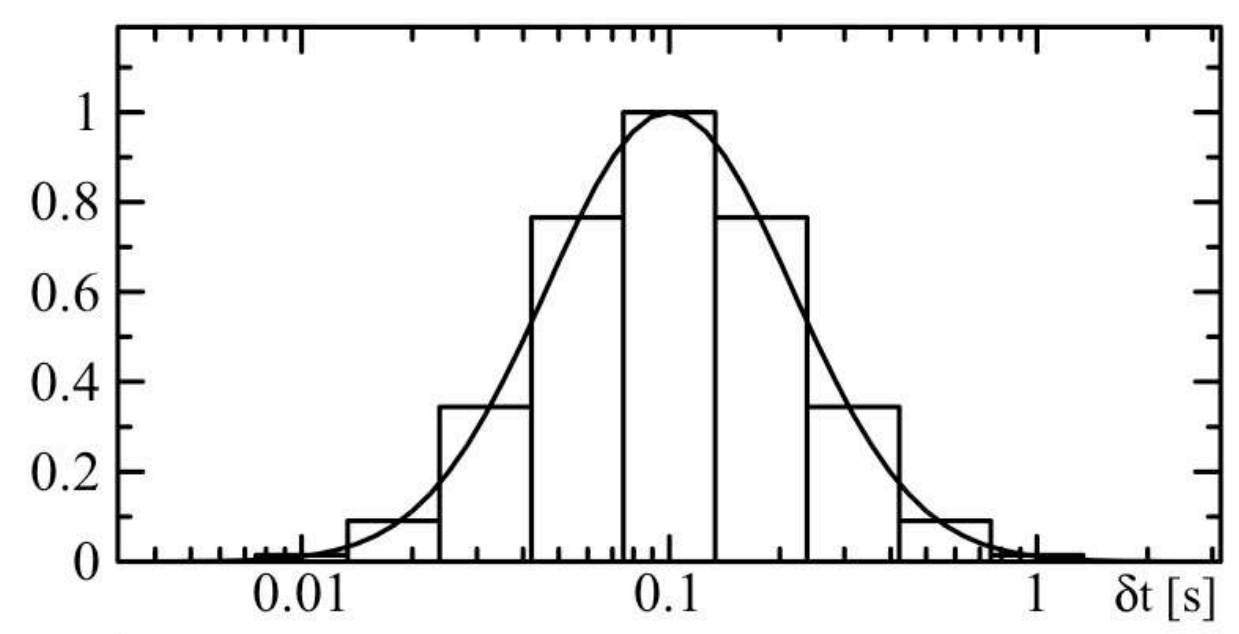

- Assume Internal Shock model but allow for shell motion and expansion, over $R_{0}-30 R_{0}$.

- Use Nakar-Piran'02 variab. time distrib, and WandermanPiran' I0 Lum. distrib., $z<6$

- Initial radius $\mathrm{R} 0$ dep. on $\delta \mathrm{t} ; \mathrm{ph}$. sp. assumed Band, obeying $E_{p}-$ $L_{\text {iso }}$ Yonetoku-Nava relatn.

- Inject protons $\mathrm{E}^{-2}$ spectrum, $\Gamma=300, f_{p}=10$ as benchmark

- MC cascades, 2 cases: neutron conversion and sudden release 


\section{Spectra at source}

Asano \& Mészáros, ApJ acc., arX:I 402.6057

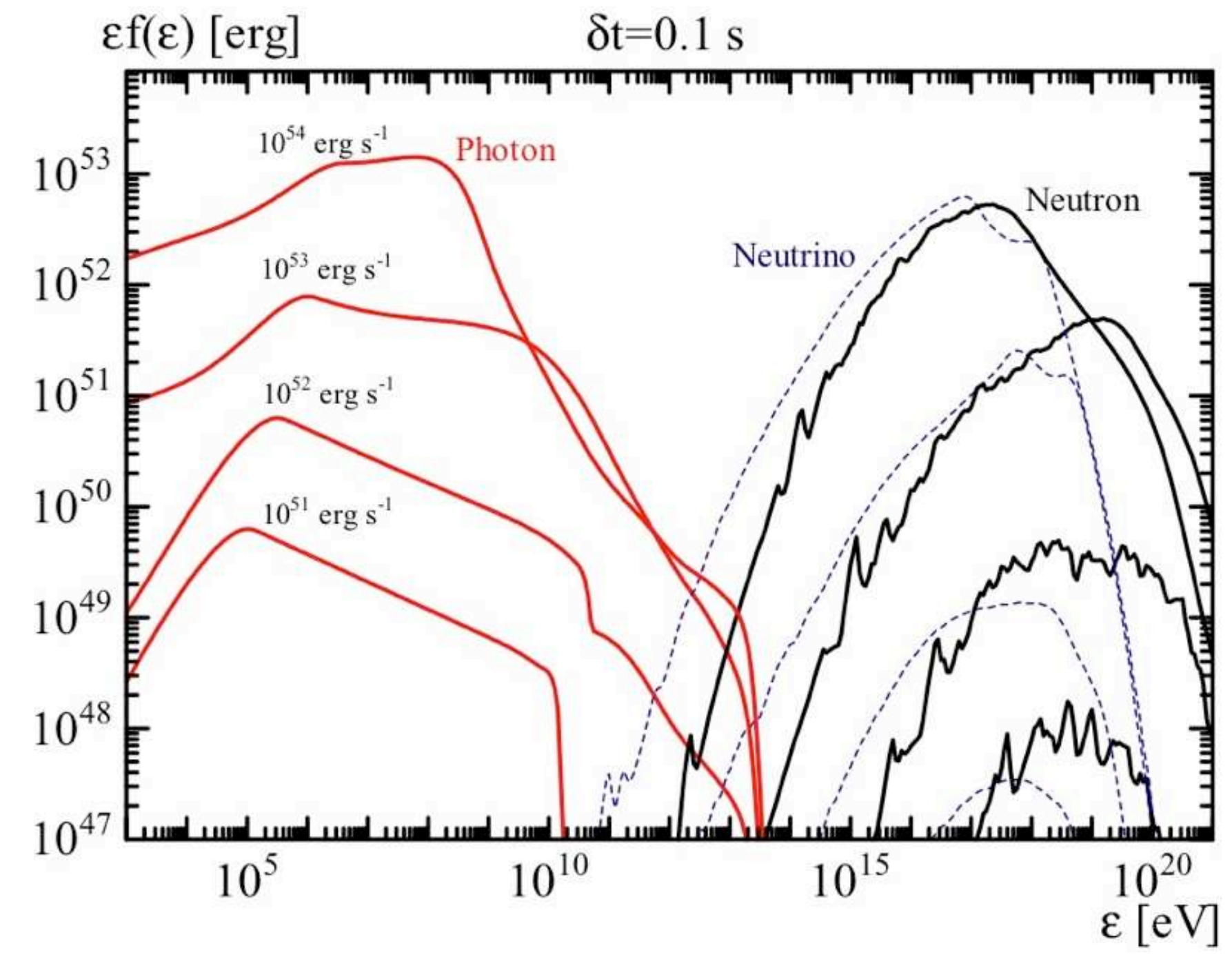

- Time integrated spectra from one shell (at source)

- Shown for $\delta \mathrm{t}=0 . \mathrm{I} \mathrm{s}$ and various lumin, for theneutron conversion model

- Blue is $v_{\mu}+v_{e}$ and their anti-flavors, before oscillation 


\section{CR + nu Diffuse Bkg}

Asano \& Mészáros, ApJ acc., arX:| 402.6057

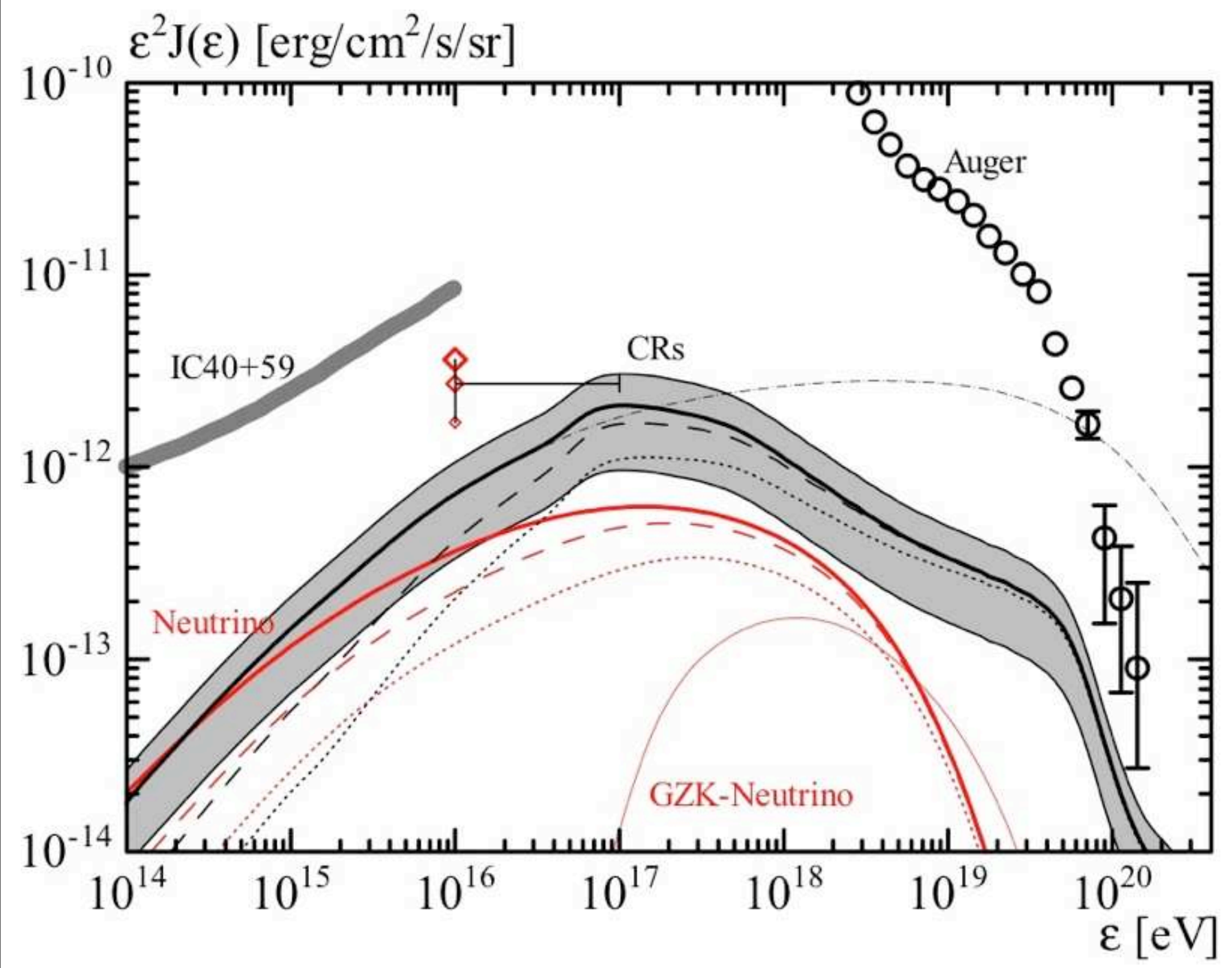

- Diffuse CR (black) and $v_{\mu}+a n t i-v_{\mu}$ (red, after osc) for neutron conversion model.

- Thin dash-dot: $\mathrm{CR}$ w/o photomeson \& BetheH ; Dash(dot): CR/V w/o GRB L > $10^{54}\left(10^{53.5}\right) \mathrm{erg} / \mathrm{s}$

- Thin red: cosmogenic nu

- Gray thick: IC3 40+59 diffuse UL for brokenPL

- Diamond: our integrated flux assuming broken PL \& uncert. break range 


\section{CR + nu Diffuse Bkg}

Asano \& Mészáros, ApJ acc., arX:| 402.6057

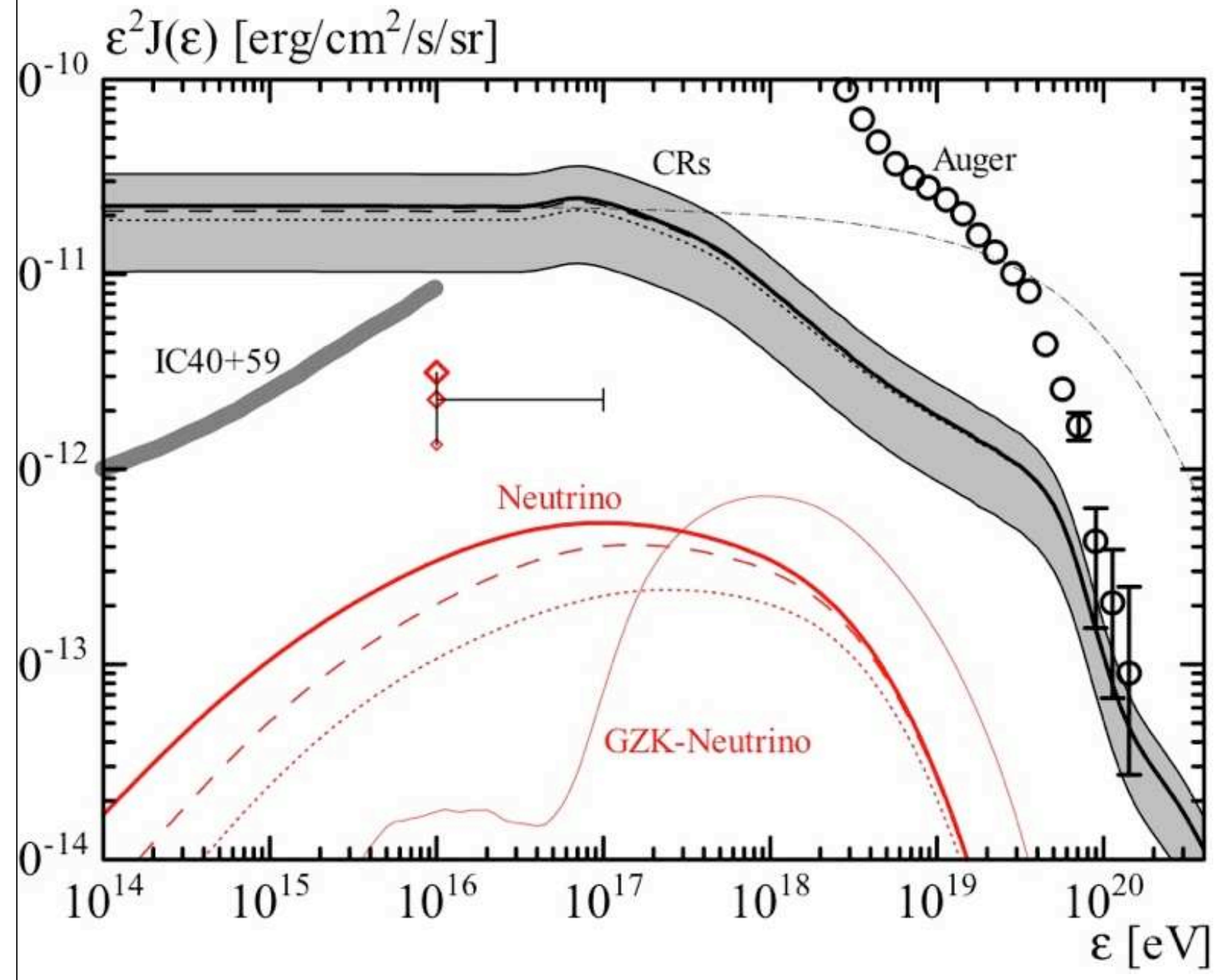

- Diffuse CR (black) and $v_{\mu}+a n t i-v_{\mu}$ (red, after osc) for sudden release model.

- Thin dash-dot: CR w/o photomeson and $\mathrm{BH}$; Dash(dot): $C R / V$ w/o GRB L > $10^{54}\left(10^{53.5}\right) \mathrm{erg} / \mathrm{s}$

- Thin red: cosmogenic nu

- Gray thick: IC3 40+59 diffuse UL for brokenPL

- Diamond: our integrated flux assuming broken PL \& uncert. break range 


\section{In other words:}

- IceCube neutrino ULs on the diffuse nu-bkg do not constrain the GRB IS model so far; will need several years to get near the ULs

- IceCube does not constrain the GRB IS model's ability to produce $10^{19}-10^{21} \mathrm{eV}$ UHECR

- Even moderate $L \sim 10^{53} \mathrm{erg} / \mathrm{s}$ GRBs with $\mathrm{f}_{\mathrm{p}} \sim 10$ are able to explain GZK CRs (but below the ankle need other sources- known this for long)

- Thus, GRBs do not explain the diffuse PeV nus

- But GRBs may contribute significantly to the observed flux of $10^{19}-10^{21}$ eV UHECR 


\section{More recently, late 2013: ICECUBE announced}

The first detection of "certified" astrophysical neutrinos 


\section{The PeV INB-IGB Connection: GRBs? AGNs? SFGs? HNe? GMSs?}

- PeV nu INB obs. by IC3 is $\sim 10^{-8} \mathrm{GeV} / \mathrm{cm}^{2} / \mathrm{s} / \mathrm{sr}$, but IC3 limit on GRB nus is factor $\sim 10$ below ("standard" IS or photosphere- ICRCI3) $\rightarrow$ could be EM dim/nu-bright GRBs? ( Liu \&Wang I3, ApJ 766:73, Murase \& loka, I3, PRL III:121102)

- PeV nu INB from hadronic low lum. AGNs: scaling $L_{p}$ from $L_{e}$ via $\mathrm{L}_{\text {phot, }}$, argue that $\boldsymbol{F R I} \mathbf{R G s}$ (higher density knots) reproduce via $\mathbf{p} \boldsymbol{p}$ the PeV nu bkg (Becker Tjust, arXiv:I406.0506) $\rightarrow$ also IGB?

- PeV nus from individual bright radio-gamma AGNs (blazars in TANAMI sample), interpreting $X-\gamma$ flux as due to $P \gamma$ photohadronic interactions, conclude that 6 of these blazars within I $\sigma$ error box of the three PeV events could account for the INB (Krauss, et al, I406.0645) $\rightarrow$ IGB?

- Starburst galaxies (SBGs), if responsible for PeV nu INB via Pp, can contribute $\sim \mathbf{2 0} \%$ of the gamma background (IGB) (Chang et al, 1406.1099) 


\section{IGB (Fermi) \& resolved sources}

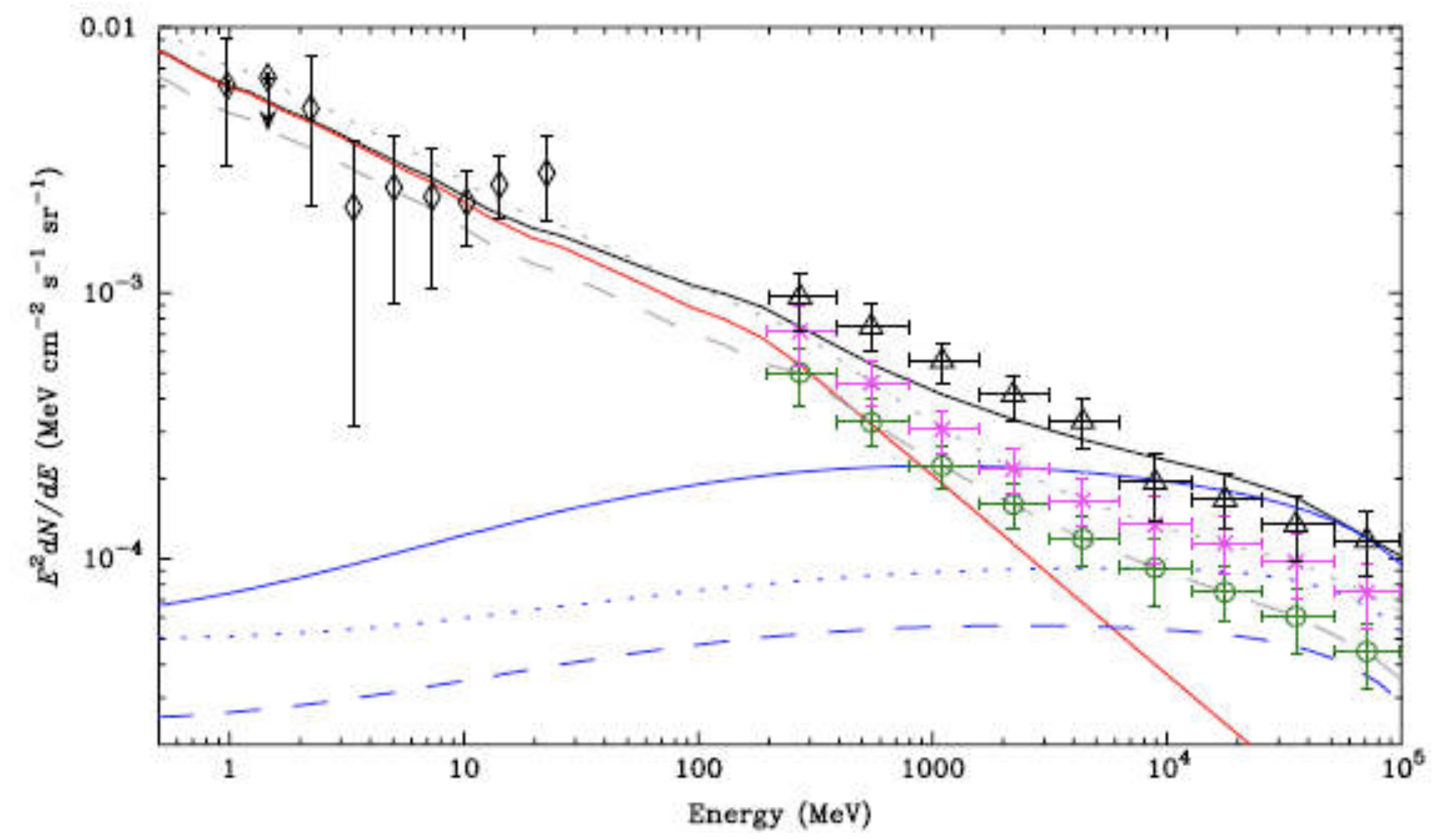

Abazajian+I I, PRD 84:103007

- Black triangle: Fermi IGB spectrum, Abdo+20 I0, PRL 104:10 I I0I

- Red line: FSRQ, blue line: BL Lac contributions

- Magenta star/green circle: upper/lower 95\% CL forecast of FermiLAT 95\% CL 5 year sensitivity 


\section{INB \& IGB from pP sources}

Murase,Ahlers, Lacki 13

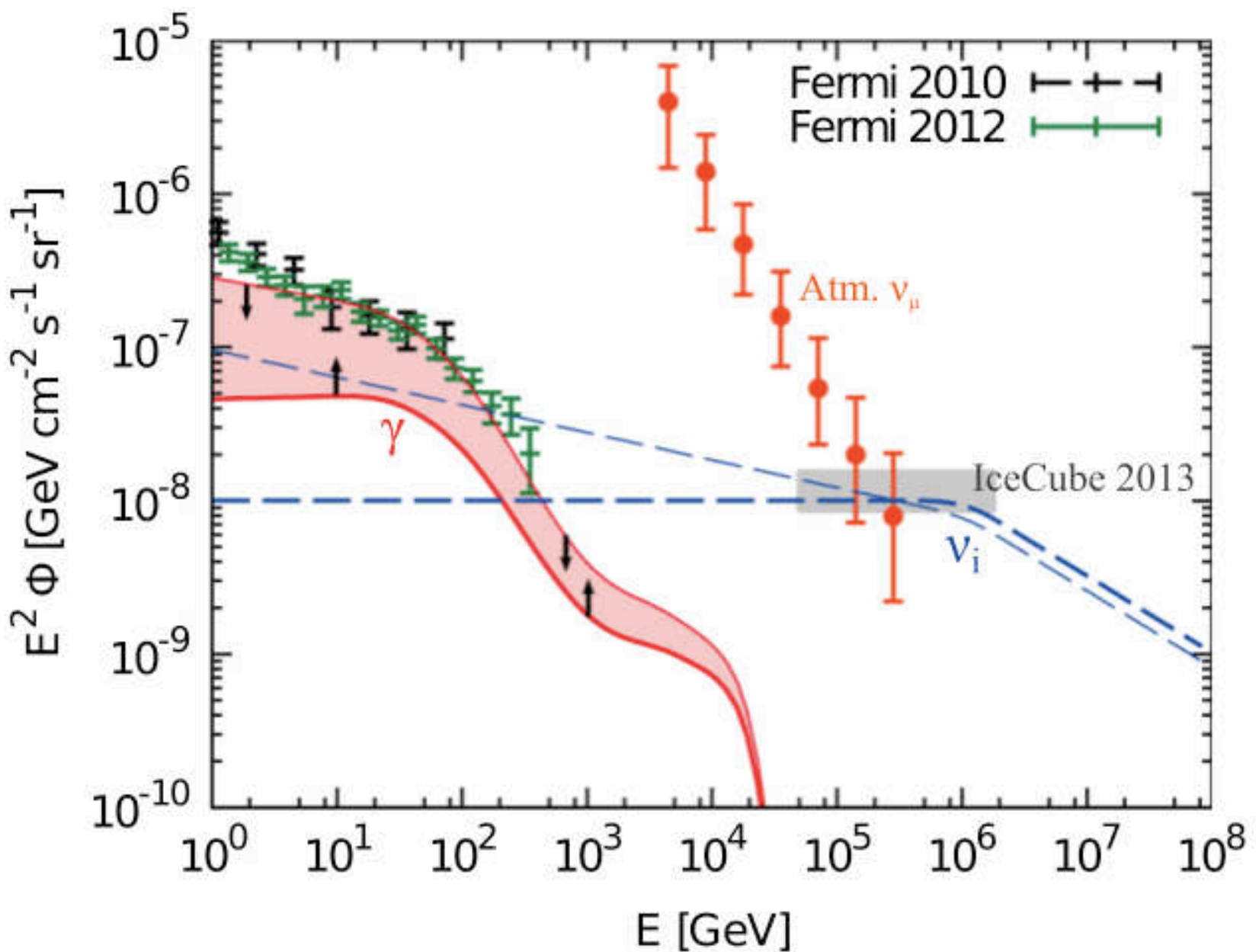

PRD 88, I 2 | 30 |

- Stress pp vs. py because no $>$ GeV threshold

- Use IC3 det. of PeV Vs, consider $\pi^{ \pm} \rightarrow V$ DNG \& $\pi^{0} \rightarrow 2 \gamma$ IGB \& satisfy Fermi/LAT bound, also lack of Glashow reson.

- Conclude $\Gamma_{\mathrm{p}} \sim 2.0-2.18$ with cutoff $<3-4 \mathrm{GeV} \boldsymbol{V}$

- Sources could be galaxy cluster shocks (IGS) or SFG/SBG - cutoff may be $t_{\text {diff }} \sim \mathrm{t}_{\text {inj }}\left(\right.$ or $\left.\mathrm{t}_{\text {diff }} \sim \mathrm{t}_{\mathrm{pp}}, \mathrm{t}_{\mathrm{adv}}\right)$

Kashiyama \& Mészáros, I 405.3262 


\section{SFG-SBG and the IGB}

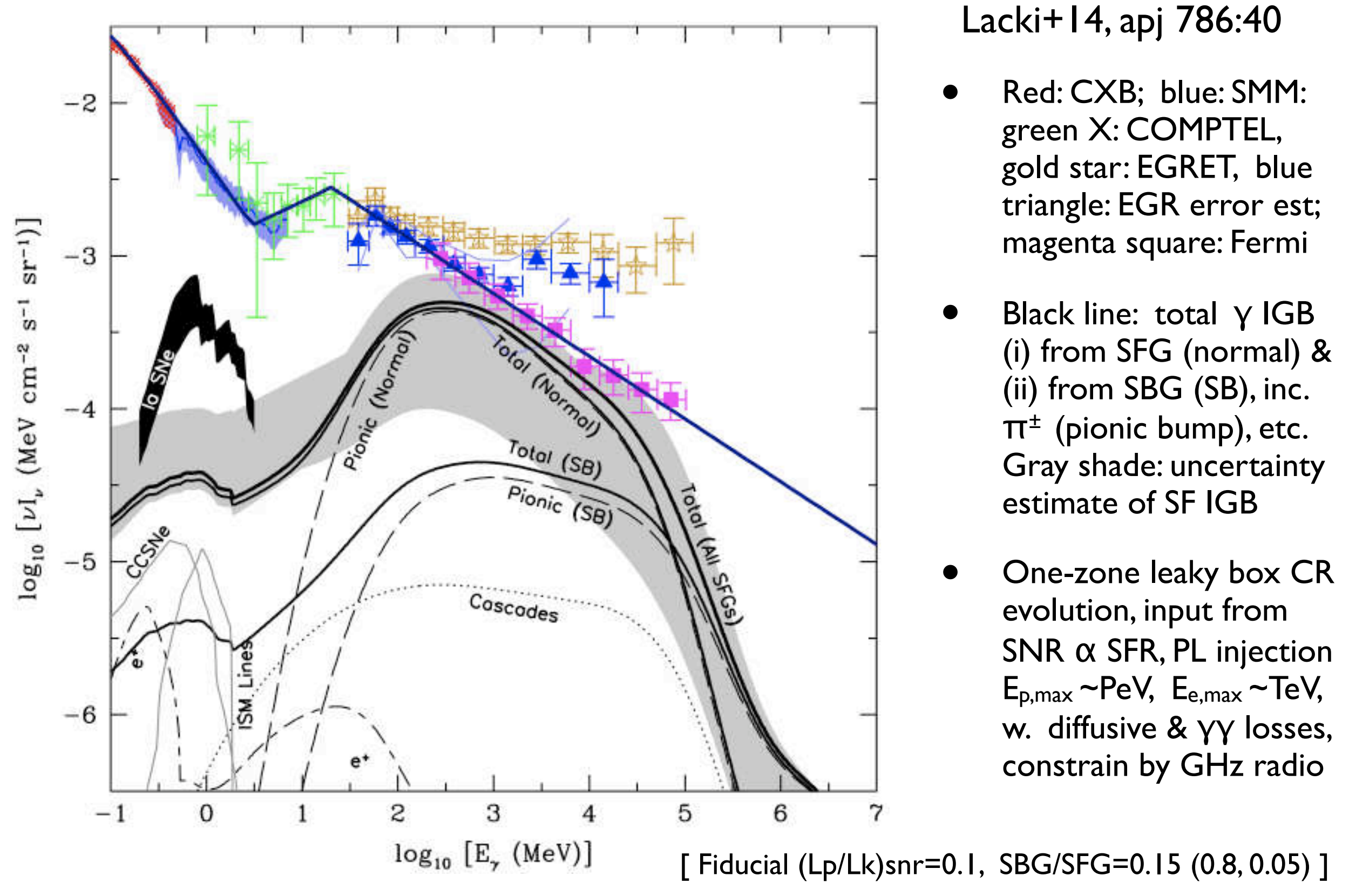




\section{SBG \& IGB - host sy losses}

Chang \& Wang, | 406.1988

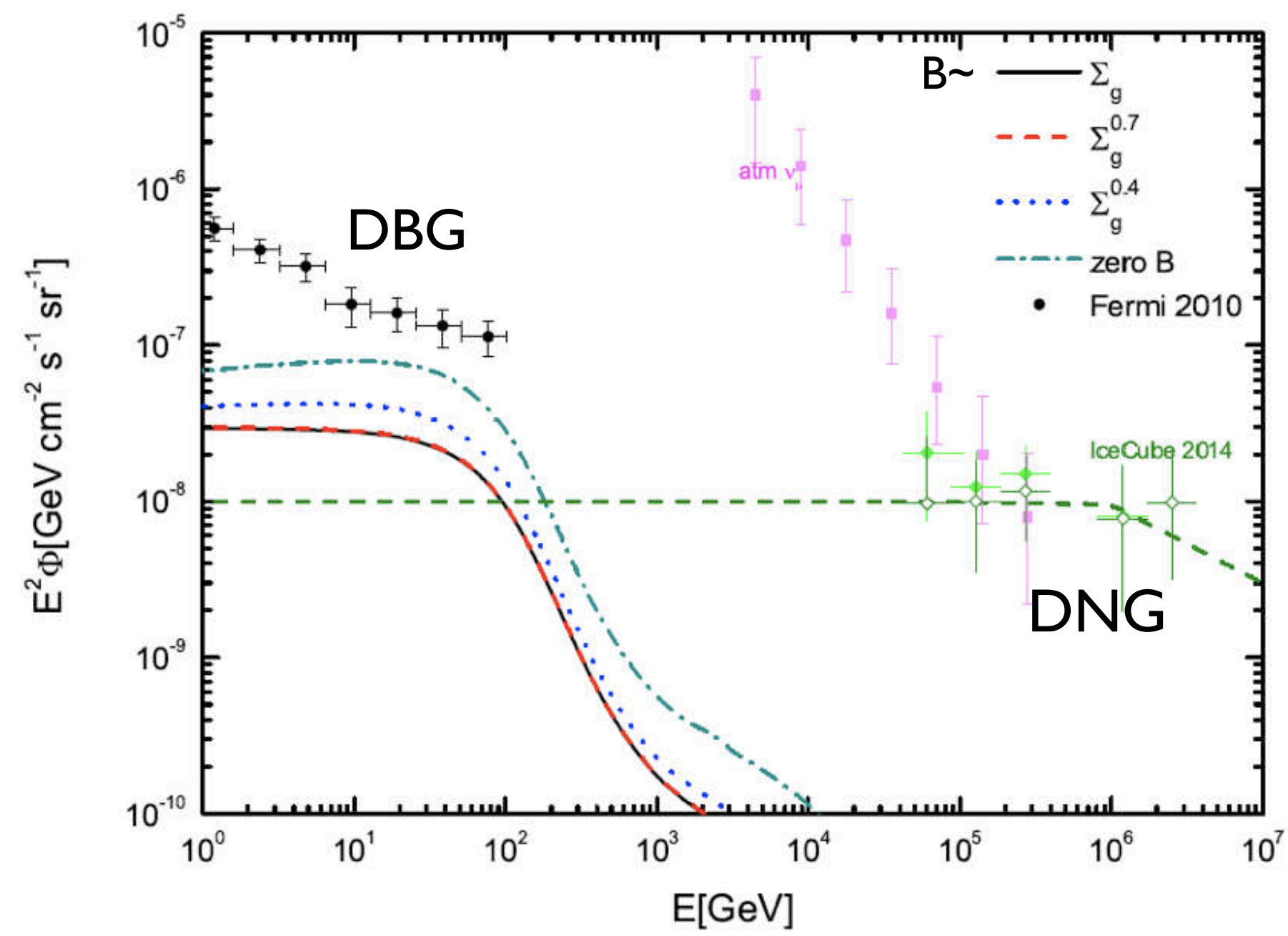

- Calibrate $\pi^{0} \rightarrow 2 \gamma$ flux using IC3 PeV nu obs. flux,

- Assume due to SBG

- Inside host galaxy, consider $Y Y$ casc. of primary $\pi^{0} \& \pi^{ \pm} I C$ upscatt. photons.

- If no sync. losses, $\Phi_{\gamma, \mathrm{casc}} \sim 0.5 \Phi_{\gamma, \mathrm{dbg}}$

- If incl. sync.losses inside host SBG (B mG) then $\Phi_{\mathrm{Y}, \text { casc }} \sim 0.2 \Phi_{\mathrm{Y}, \mathrm{igb}}$

However: if IGB \& INB arise in less excited galaxies (e.g. SFGs), $B_{\text {ism }}$ may be smaller $\rightarrow$ the sy losses are smaller, and $\Phi_{\gamma, \text { casc }}$ larger 


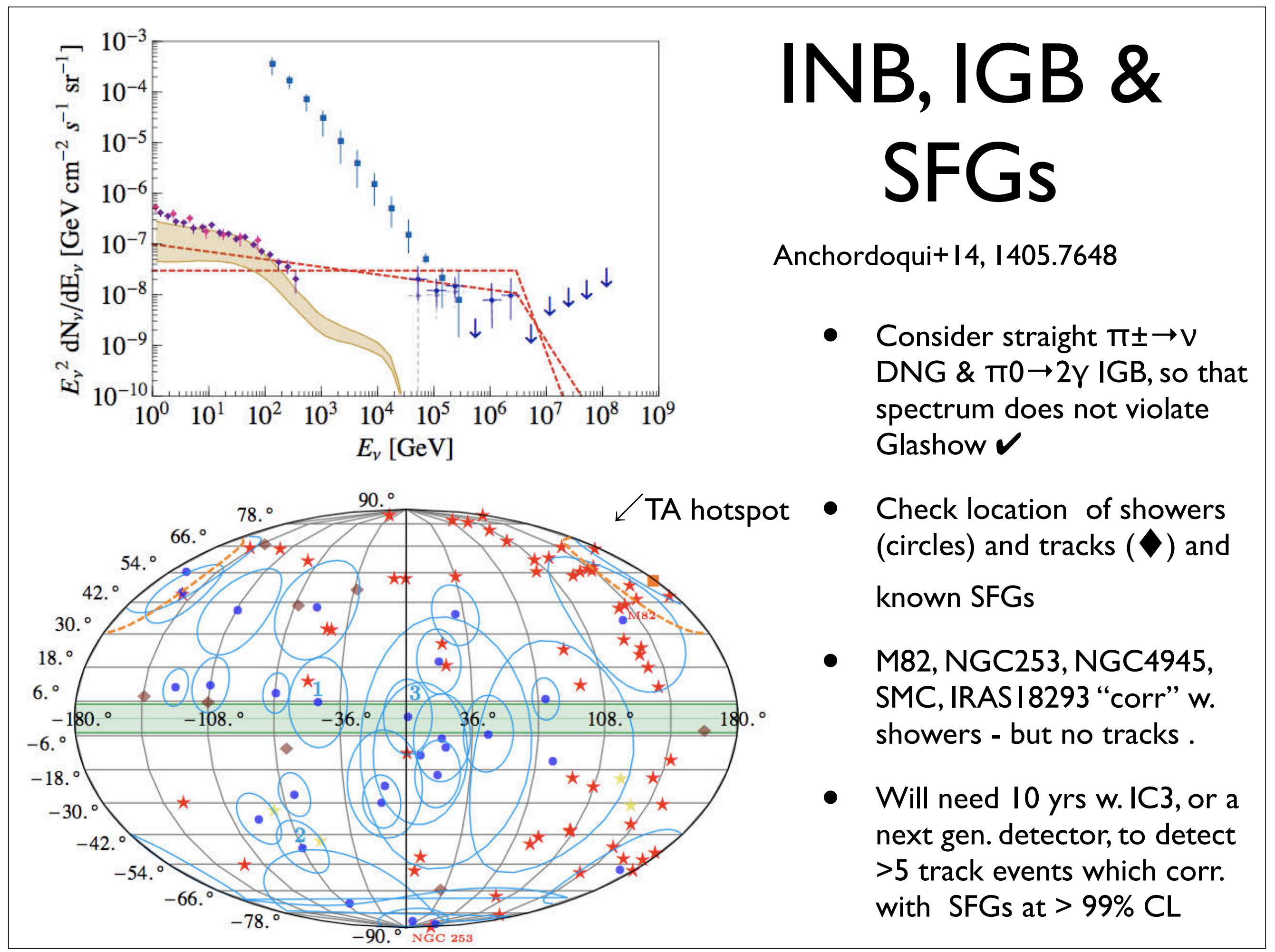




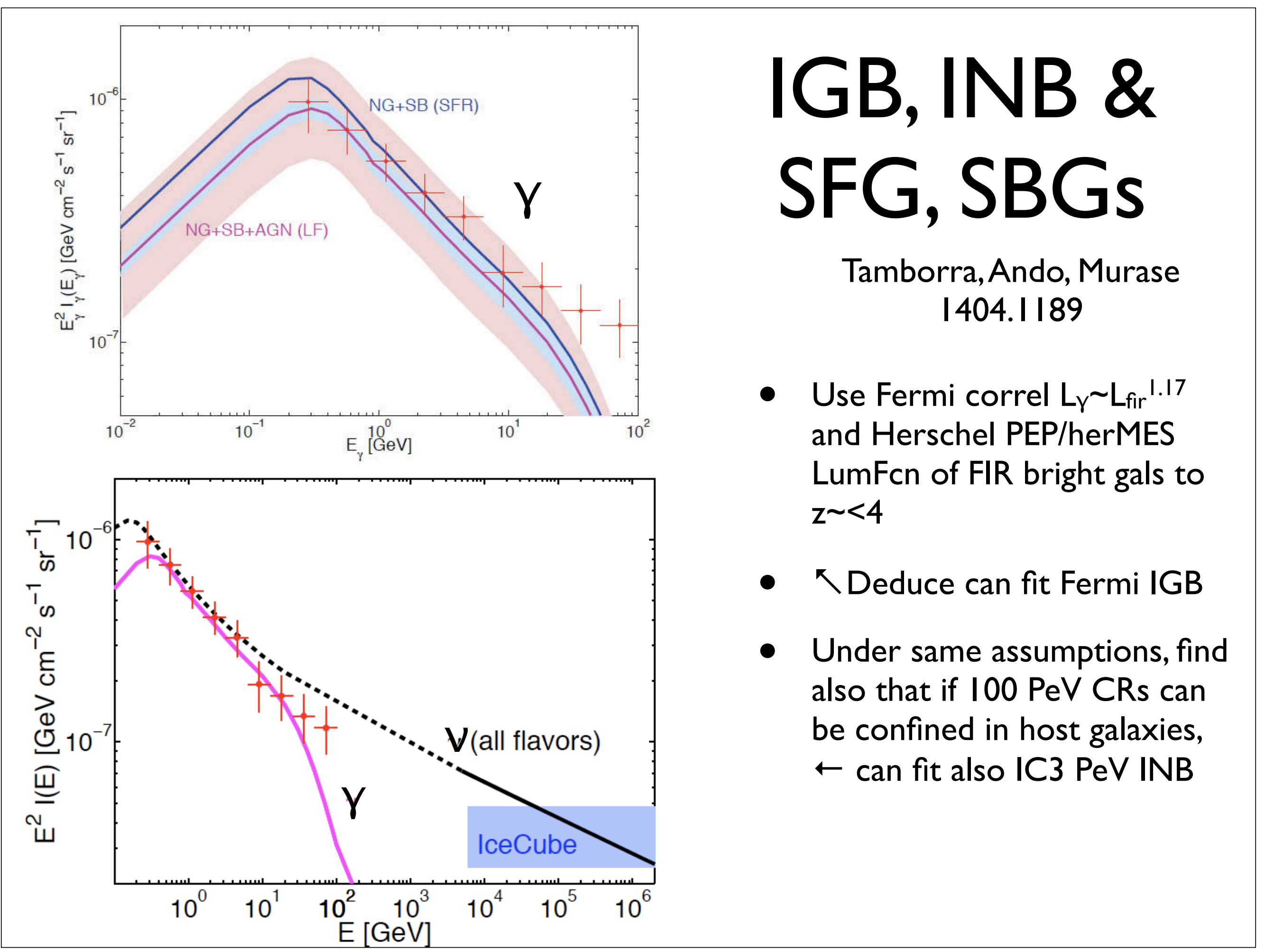




\section{Galaxy mergers, INB \& IGB}

Kashiyama \& Mészáros, I405.3262

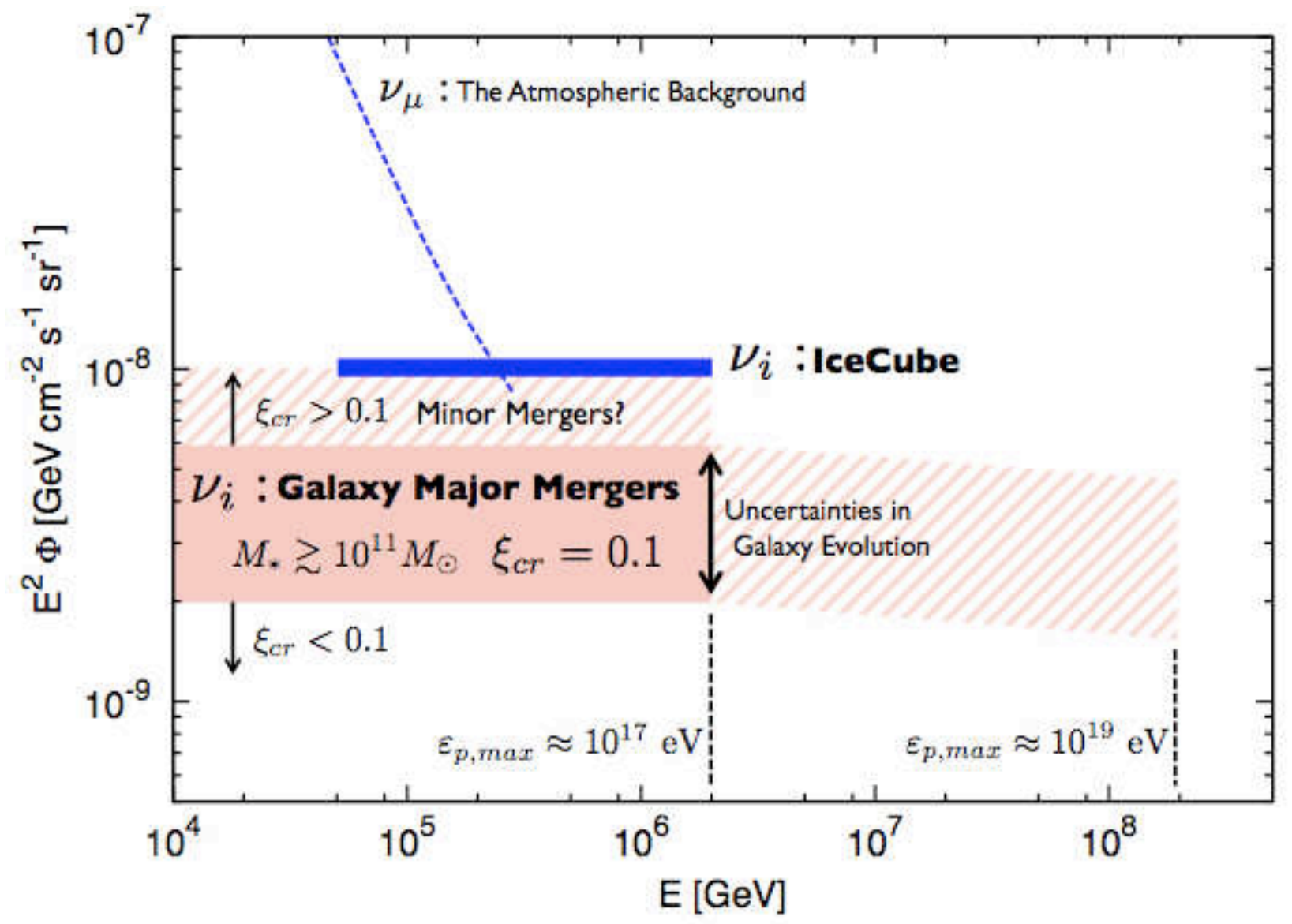

- Every galaxy merged at least once in the last Hubble time

- Major mergers $\rightarrow$

$E_{\text {gms }} \sim 10^{58.5} \mathrm{erg}$, $R \sim 10-4 \mathrm{Mpc}^{-3} \mathrm{Gyr}^{-1}$ $\mathrm{v}_{\mathrm{s}} \sim 10^{7.7} \mathrm{~cm} / \mathrm{s}$ $\mathrm{Q}_{\mathrm{cr}, \mathrm{gms}} \sim 3 \times 10^{44} \mathrm{erg} \mathrm{Mpc}^{-3} \mathrm{yr}^{-1}$ $\varepsilon_{\text {cr,max }} \sim 10^{18.5} \mathrm{Z} \mathrm{eV}$

- $\quad \boldsymbol{p p} \rightarrow \mathrm{PeV}$ vs, $100 \mathrm{GeV}$ Ys

- v: Indiv. GMS: $0.01 \mu / y r$, INB: 20-60\% IC3 obs.flux

- $\quad y$ : Individual GMS flux: $\sim 3.10^{-13} \mathrm{erg} / \mathrm{cm} 2 / \mathrm{s}$, CTA? IGB $\sim 10^{-8} \mathrm{GeV} / \mathrm{cm}^{2} / \mathrm{s} / \mathrm{sr}$, about 10-30\% Fermi IGB

- Minor mergers: uncertain, could add up to $70-100 \%$ 


\section{Outlook \& Issues}

- Both in AGNs and GRBs, major question is whether basic emission is leptonic or hadronic - contribution to the observed CRs/UHECRs and PeV nus?

- Location of the $\mathrm{GeV}(\mathrm{TeV})$ emission region (inner/outer jet, photosphere?) Role of (which?) target photon sources

- Role of pair cascades in VHE spectrum formation

- Do galaxy/cluster shocks and/or galaxy merger shocks contribute much (all?) of SFG/SBG VHE radiation?

- Relative contribution of AGNs, SFG/SBG/GMS to the IGB and/or the INB? is pp, py or leptonic dominant in $Y$ ? 


\section{Outlook (continued)}

- The sources of the UHECR are still unknown..!

- They are almost certainly astrophysical sources (not TD)

- $G R B$ remain good candidates, as well as $A G N s, H N e, R Q$, maybe MGRs.

- Will increasingly constrain such possibilities with GeV and $\mathrm{TeV}$ photon observations

- Will learn even more if \& when astrophysical UHENUs are observed from any type of (individual) source

- Constraints from diffuse (and intrasource) $y$-ray and nu emission will also be very useful, and may remain for a long time the main constraint

- Composition and clustering will provide important clues 